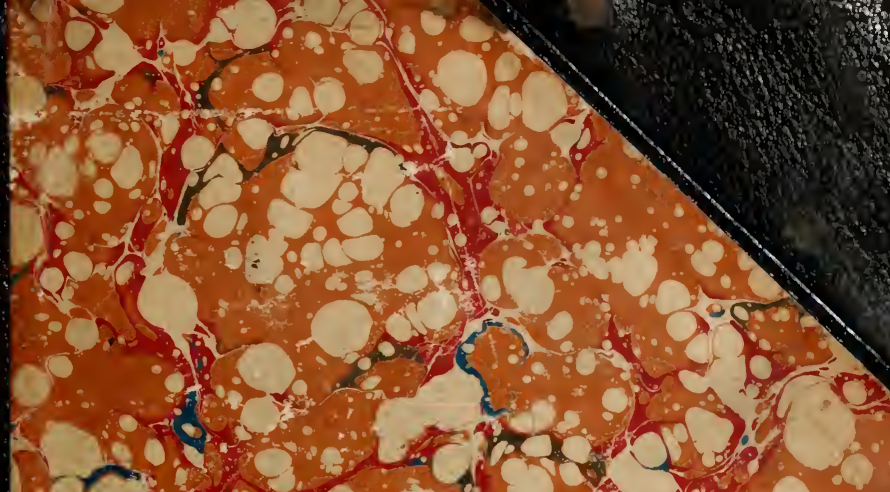

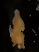

10 - 5.

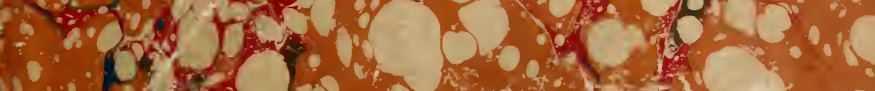

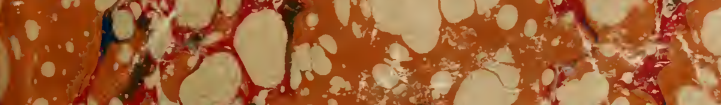

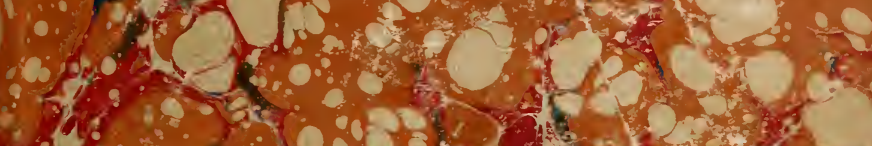

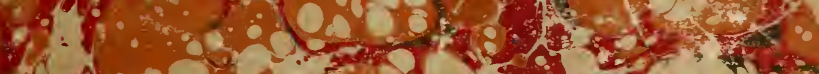

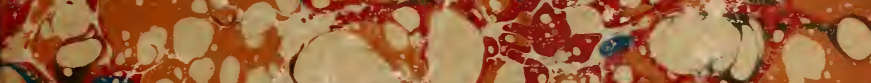

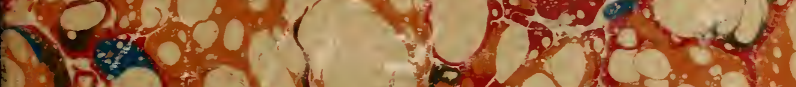

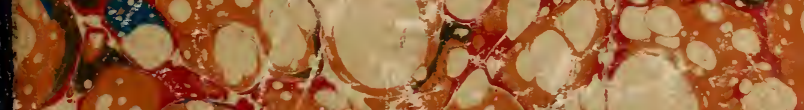

E. 13 (3)

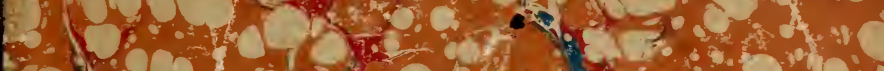

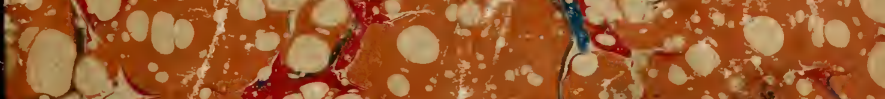

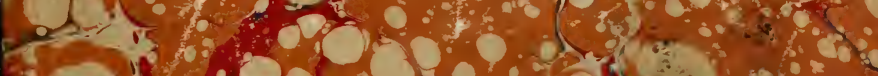
Ro 500 a 200 $\left(\begin{array}{ll}0 \\ 0 \\ 0\end{array}\right.$ 



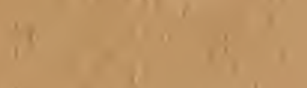

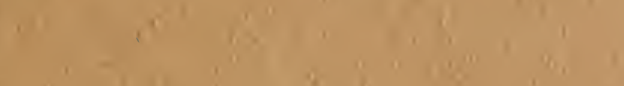

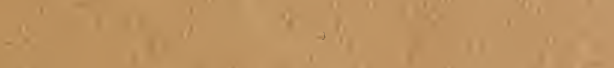

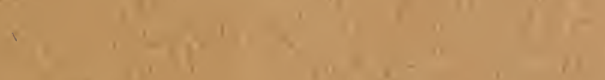

$x+1$

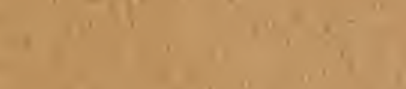

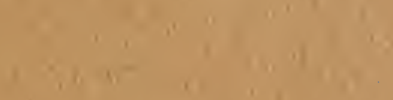

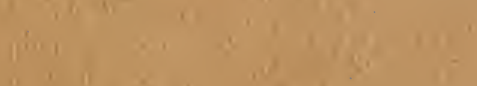

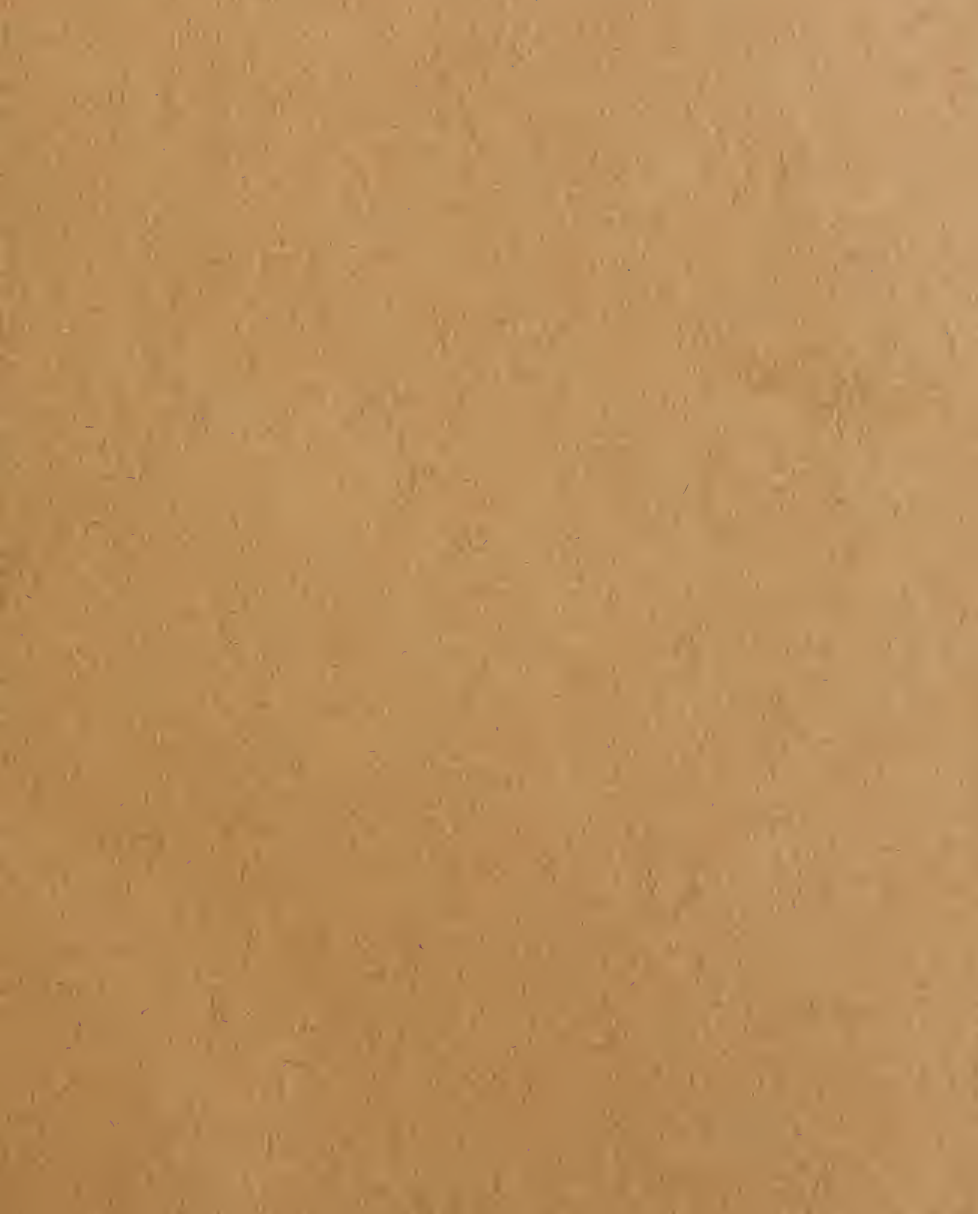




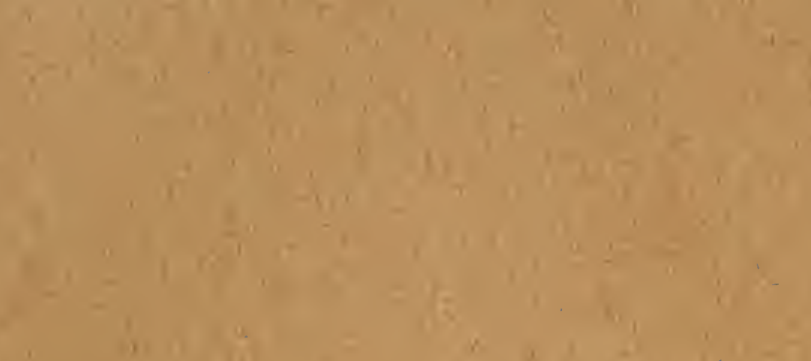

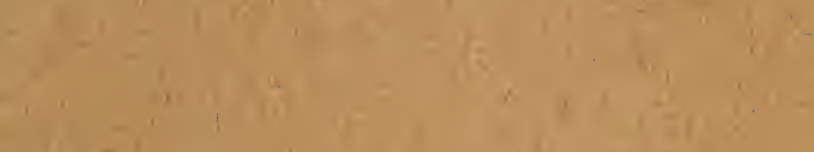
$y=$

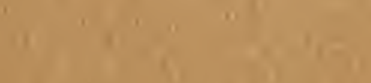

, 1,1

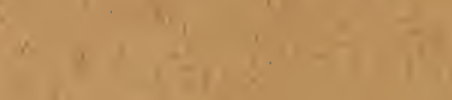
$\frac{1}{7}+1$

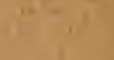

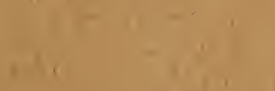

(1)

$\frac{1}{3}+\frac{1}{1}$

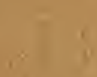

$8 x+\frac{7}{9}+1$

$P^{\prime}=1+1$

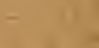

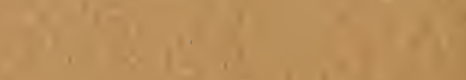

$+18$

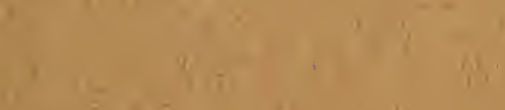

$x+2, \frac{1}{3}+1$

11

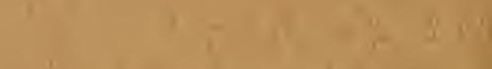

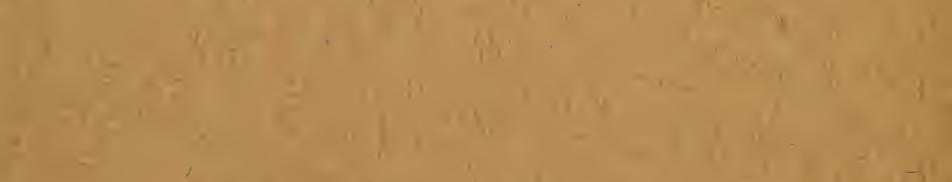

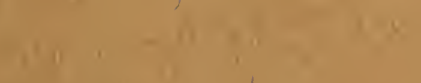

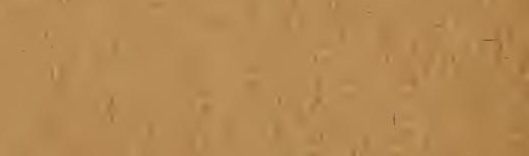

S)

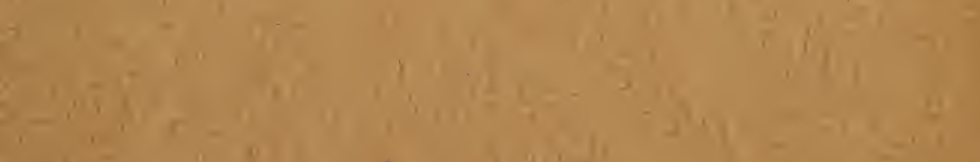

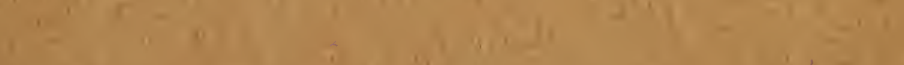

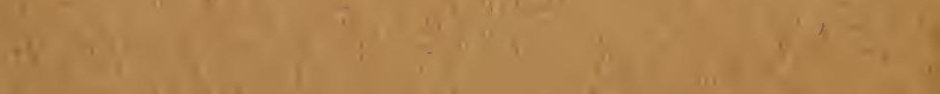

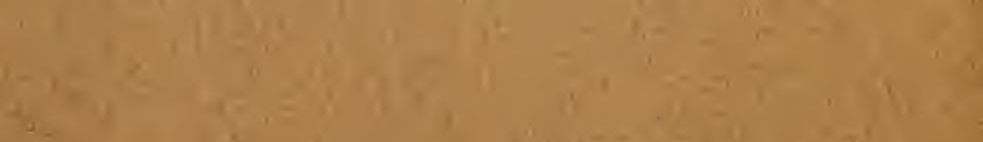

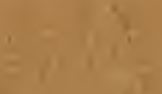

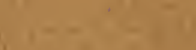
$\left(x+\frac{1}{1}\right.$ 


\section{LA}

SCIENCE EXPÉRINENTALE 


\section{TRAVAUX DU MEME AUTEUR}

Cours de Médecine du Collége de France

Leçons de physiologie expérimentale appliquée à la médecine. Paris, 185 t-185ั, 2 vol. in-8 avec tigures. $14 \mathrm{fr}$.

Leçons sur les effets des substances toxiques et médicamenteuses. Paris, 1857, 1 vol. in-8 avec figures. $7 \mathrm{fr}$.

Leçons sur la physiologie et la pathologie du système nerveux. Paris, 1858, 2 vol. in-8 avec figures. $14 \mathrm{fr}$.

leçons sur les propriétés physiologiques et les altérations pathologiques des liquides de l'organisme. l'aris, 1859, 2 vol. in-8 avec 22 figures. $14 \mathrm{fr}$.

Lecons de pathologie expérimentale. Paris, 1871, 1 vol. in-8 de 600 pages.

$7 \mathrm{fr}$.

Leçons sur les anesthésies et sur l'asphyxie. Paris, 1875̃, 1 vol. in-8 de 600 pages avec figures.

$7 \mathrm{fr}$.

Leçons sur la chaleur animale, sur les effets de la chaleur el sur la fièvre. Paris, 1876,1 vol. in-8 de 372 pages avec figures.

$7 \mathrm{fr}$.

Leçons sur le diabète et la glyeogénèse animale. Paris, 1877,1 vol. in-8, vilI-576 pages avec figures.

$7 \mathrm{fr}$.

Cours de Physiologie générale du Huséum d'Histoire naturelle

Leçons sur les phénomènes de la vie communs aux animaux et aux végétaux. Paris, 1878, 1 vol. in-8 de 4 jo pages avec une planche coloriée et 44 figures. $7 \mathrm{fr}$.

- Tome II (sous presse).

Introduction à l'étude de la médecine expérimentale. Paris, 1865, 1 vol. in-8 de 400 pages.

$7 \mathrm{fr}$.

Précis iconographique de médecine opératoire et d'anatomie ehirurgieale, par Cl. Bernard el Huette. Paris, 1073,1 vol. in-18 jésus de 495 pages arec 113 pl., figures noires. Cartonné.

$24 \mathrm{fr}$.

- Le même, fig. coloriées.

$48 \mathrm{fl}^{\mathrm{l}}$,

Fr. Magendie. Paris, 1856. In-8.

$1 \mathrm{fr}$.

Typographie Lahure, rue de Fleurus, 9, à Paris. 


\section{LA}

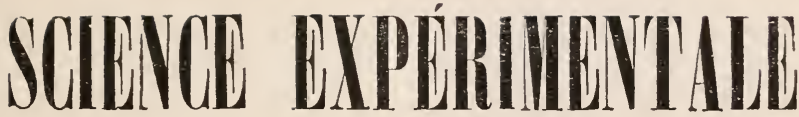

PAR

\section{GLAUDE BERNARD}

MEMBRE DE L'INSTITUT

(Académie des Sciences et Académie irançaise)

PROFESSEUR A U MSÉM D'HISTOIRE NATURELLE

ET AU COLLÉGE DE FRANGE

Avec figures intercalées dans le texte

Progrès des sciences physiologiques

Problèmes de la physiologie générale

La vie, les théories anciennes

et la science moderne

La chaleur animale, la sensibilité

Le curare, le cour, le cerveau

Discours de réception à l'Académie francaise.

\section{PARIS}

LIBRAIRIE J.-B. BAILLIÈRE \& FILI

19, RUE HAUTEFEUILLE, PRÈS DU BOLLEVARD SALNT-GERMAIN

1878

Tous droits réservés 



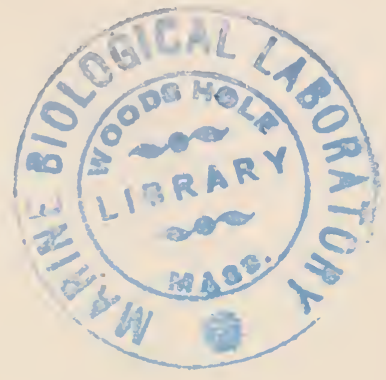

M. Dumas, vice-président du Conseil supérieur de l'Instruction publique, secrétaire perpétuel de l'Académie des Sciences, membre de l'Académie française, a prononcé aux funérailles de $\mathbf{M}$. Claude Bernard, le 16 février 1878, le discours suivant :

\section{Messieurs,}

Le Conseil supérieur de l'Instruction publique réclame une large part dı deuil qui frappe si douloureusement l'Université, l'Institut et la France; lorsqu'on voit s'é- 
teindre une des grandes lumières du pays, il perd toujours un des siens, et le Vinistre éminent qui le préside a voulu que je vienne en son nom déposer sur cette tombe l'expression de nos regrets.

Claude Bernard que nous pleurons, s'était placé par son rare génie et par ses brillantes découvertes à cette hauteur où l'on cesse d'appartenir exclusivement à une compagnie et même à une nation, pour prendre rang dans le concert de la science universelle; vivant, sa gloire avait franchi l'espace, elle était acclamée par le monde entier; mort, elle bravera le temps et ses outrages.

Après Lavoisier, Laplace, Bichat, Magendie, qui lui avaient ouvert la route, Claude Bernard a épuisé ses forces à son tour à l'étude du grand mystère de la vie, sans prétendre à pénétrer toutefois son origine et son essence. L'astronome ignore la cause de l'attraction universelle et n'en calcule 
pas moins avec certitude la marche des astres qu'elle sontient dans l'espace et dont elle dirige le cours. Claude Bernard avait jugé qu'il est permis de même, au physiologiste, d'expliquer les phénomènes de la vie, au moyen de la physique et de la chimie qui exécutent, quoique la vie et la pensée, qui dirigent, demeurent hors de sa portée.

La physique animale n'était-elle pas fondée en effet, dès que Lavoisier et Laplace eurent prouvé que la respiration est une combustion, source de la chaleur qui nous anime? Ce flambeau de la vie qui s'allume, cette flamme de la vie qui s'éteint, expressions poétiques heureuses de l'antiquité, ne devenaient-elles pas des vérités philosophiques, auxquelles il a été donné à Claude Bernard d'ajouter le dernier trait?

L'anatomie générale n'était-elle pas née le jour où Bichat définissait la vie : "l'en- 
semble des fonctions qui résistent à la mort? „Sans en révéler la secrète nature, n'apprenait-il pas à préciser les formes que la vie revêt dans chacun des éléments dont se composent nos tissus, à considérer comme l'expression sensible de la vie, ces mouvements de destruction et de rénovation dont ils sont le théâtre; leur arrêt comme le signe certain de la mort?

Magendie n'ouvrait-il pas enfin la route à la physiologie expérimentale, devenue entre ies mains de Claude Bernard, son élève, une science nouvelle? Empruntant à la physique et à la chimie ses instruments et ses méthodes, sans oublier que les forces dont elles disposent vont s'exercer sur des êtres doués de vie, n'est-ce pas Claude Bernard qui l'a portée au rang des sciences exactes et qui la laisse rivalisant de certitude et d'autorité avec celles qui opèrent sur la matière brute?

Parmi tant de découvertes, auxquelles 
son nom demeure attaché, quelle merveille de sagacité et d'analyse que ce travail à jamajs célèbre et depuis longtemps populaire où, donnant un corps certain à la pensée de Bichat, il fait voir dans le muscle qui se contracte, dans le nerf qui le met en mouvement, dans l'élément nerveux sensitif et dans l'élément moteur, autant de modes distincts de la vie, pouvant coexister, mais aussi pouvant mourir séparément et comme en détail !

Quel physiologiste ne serait fier d'avoir découvert la véritable fonction du foie, problème qui depuis l'antiquité la plus haute jusqu'à nos jours avait excité, mais en vain, la curiosité de toutes les écoles médicales ? Quel chimiste n'eût considéré comme un fleuron à sa couronne, cette analyse hardie et savante par laquelle Claude Bernard découvre, dans cet organe éuigmatique, une matière propre à se changer en sucre, un ferment capable d'en opérer la conversion, 
une source enfin qui verse sans cesse du sucre dans le saug?

Mais, je m'arrête et je laisse à des voix plus autorisées le droit d'exposer dans toute leur fécondité les découvertes que nous devons à l'illustre physiologiste que nous venons de perdre.

S'il était permis d'éteindre, tout à coup, les lumières que la science de la vie emprunte anx travaux de Lavoisier, de Laplace, de Bichat, de Magendie et de claude Bernard, l'esprit humain reculerait de dix siècles.

Les phénomènes physiques de la vie n'ont plus dinaccessibles secrets. Les problèmes qui s`y rapportent ont tous été abordés par Claude Beruard avec confiance, poursuivis avec obstination. Il en est peu qu'il n'ait résolus, et dont il n'ait ramené la solution, ‘ force de génie, à ces formules élégantes et simples où l'imagination du poëte se mêle à la rigueur de la géométrie. 
La France perd en Claude Bernard un de ses fils les plus illustres; la science un de ses représentants les plus respectés: nous tous, un confrère aimé, dont le commerce plein de charme et de douceur, après lui avoir acquis l'universelle sympathie, assure à sa mémoire un éternel regret.

En ce moment où des coups répétés nous frappent, où nous perdons en quelques mois, Brongniart, Balard, Le Verrier, Becquerel, Regnault, Claude Bernard, et quand la science française, presque décapitée, a besoin de tourner vers' l'avenir des regards d'espérance, les pouvoirs publics ont voulu que les honneurs réservés aux capitaines qui se sont illustrés en défendant la patrie, aux politiques qui en ont dirigé les destinées à travers les écueils, fussent aussi rendus au génie de l'étude. Ce n'est pas en vain que ce grand spectacle aura été déployé en face de nos écoles. Une noble émulation, troublant les jemnes âmes qui le con- 
templent émues, ira réveiller leur ardeur, leur inspirer l'amour de la vérité, l'ambition de la gloire et le dédain de la fortune.

Les forces morales de la France semblent menacées; préparons des successeurs à ces grands hommes, presque tous enlevés avant l'heure! Ouvrons la route à leurs émules, à ces yénies naissants que nos vœux appellent et que réclament nos rangs décimés.

Claude Bernard s'écriait, au souvenir des misères que tous les savants ses contemporains ont partagées : « L'étude de la physiologie exige deux choses: le génie qui ne se donne pas et les ressources matérielles qu'un vote des pouvoirs publics suffirait à lui assurer. La physiologie française ne réclame que des moyens de travail, le génie qui les mettrait à profit ne lui a jamais manqué. » Toutes les sciences pourraient tenir le même langage.

Adieu, Claude Bernard, vous que les 
honneurs ont toujours été chercher et qui n’en avez jamais réclamé aucun, votre cri suprême sera entendu par le ministre de l'instruction publique qui vous accompagne à votre dernière demeure. La pompe inusitée de vos funérailles apprendra de quels respects il veut que les sciences soient entourćes. Votre vie laborieuse et modeste restera comme un salutaire exemple; votre mort, glorifiée de tout un peuple, comme un enseignement.

Du sein de la vie éternelle, dont le secret vous a été révélé désormais, si votre modestie s'étonne des honneurs qui vous sont rendus, votre génie s'en reconnaît digne et votre patriotisme les accepte comme une promesse d'avenir et un gage de grandeur future pour la science française. 



\section{CLAUDE BERNARD}

La science expérimentale vient de perdre son plus éminent maître : M. Claude Bernard, membre de l'Académie des sciences et de l'Académie française, professeur au Collége de France et au Muséum d'histoire naturelle, est mort, hier soir, à la suite d'une longue et douloureuse maladie.

Le temps et la liberté d'esprit nous manquent aujourd'hui pour apprécier l'œuvre de cet homme de génie : une de nos Revues scientifiques lui sera sous peu consacrée, et ce terrain paraitra bien étroit pour le déploiement de tant de découvertes. Nous ne pouvons actuellement que dire quelques 
mots de son histoire et du rôle qu'a joué dans l'évolution des sciences expérimentales son initiative puissante.

Claude Bernard, né à Saint-Julien, près de Villefranche (Rhône), le 12 juillet 1813 , arriva à Paris en 1832, n'apportant guère comme bagage qu'une tragédie qui ne fut jamais jouée, et qu'une comédie-vaudeville qui avait eu quelque succès sur un petit théâtre de Lyon. Saint-Marc Girardin, alors suppléant de Guizot à la Sorbonne, auquel il présenta ces premiers essais, lui conseilla “ d'apprendre un métier pour vivre, quitte à faire ensuite de la poésie à ses heures »: certes, il ne se doutait guère d'avoir devant lui un futur collègue de l'Académie frauçaise. Le jeune Claude Bernard obéit à ce sage avis, et prit ses inscriptions à la Faculté de médecine.

Bien qu'il eût obtenu en 1839 le titre d'interne des hôpitaux, ce n'était rien moins qu'un élève brillant. Ses camarades ne soup- 
connaient pas ce que recélait en son vaste front cet étudiant silencieux, peu attentif aıx leçons des maîtres, et dont le calme méditatif était volontiers taxé par eux de paresse. Ce fut une révélation dont le souvenir est souvent exprimé par ceux qui survivent que ces publications sur le suc gastrique, la corde du tympan, le nerf pneumogastrique et le nerf spinal qui, tout à coup, signalèrent au monde savant un expérimentateur ingénieux et sagace, servi par une rare habileté opératoire.

Les leçons de Magendie avaient opéré cette révolution. Dès qu'il eut mis le pied dans le laboratoire du Collége de France, sa voie fut tracée. L'expérimentation hardie, bien qu'un peu désordonnée, du célèbre physiologiste, sa critique impitoyable, son scepticisme qui s'étendait jusqu'à ses propres découvertes, firent une impression profonde, créatrice, pour ainsi dire, sur l'esprit du jeune Claude Bernard. Mais l'élève, 
bien autrement puissant que le maître, ne prit de cet enseignement que ses qualités d'indépendance, et sut maintenir le doute dans les limites scientifiques. Au dédain profond pour les explications vraisemblables où se bercent les chimères séduisantes, il sut joindre sans effort le respect des faits accumulés par la tradition, la crédulité sincère en face de l'inattendu, souvent gros de découvertes, l'estime de l'hypothèse qui cherche et de la théorie qui coordonne, sans leur jamais attribuer de vie personnelle ou d'autorité; enfin, et c'est ce qui le distingue surtout de Magendie et ce qui lui a donné un caractère tout personnel, l'amour de la certitude, le sentiment profond de la loi, l'inébranlable assurance que, si les conditions de la manifestation des phénomènes vitaux sont infiniment multiples, complexes, difficiles à saisir, à rassembler, à dominer expérimentalement, elles n'en sont pas moins sûre- 
ment, impassiblement liées à ces phénomènes, sans qu'aucun élément étranger, extranaturel, saus que nul quid divinum puisse être invoqué pour l'explication des apparentes irrégularités spontanées qu'ils présentent.

C'est en ce point capital que se marqua, dès les premiers moments de sa vie scientifique, la supériorité de Claude Bernard. L'élève du sceptique Magendie est l'introducteur du déterminisme dans le domaine de la physiologie. Grâce à lui, la méthode expérimentale, qui, si l'on en respecte les règles, mène à la certitude dans les sciences de la matière morte, a pris la même autorité dans celles des êtres vivants. Il n'y a pas deux ordres de sciences, les unes fières et assurées, les autres hésitantes et timides, les unes sûres de commander seules et d'être obéies seules par l'expérience, les autres toujours en crainte d'une intervention inconnue daus son essence, sa force et son but. 
Et les efforts ne furent pas petits qu'il fallut déployer pour bannir du terrain de la physiologie cette inconnue menaçante. Le plus célèbre des physiologistes français, Bichat, lui avait donné droit de cité. Et depuis lui, chacun avait cru devoir compter avec cette puissance capricieuse, avec ces fonctions vitales, dont le rôle était de rẻsister aux lois générales de la matière, et qui faisaient ainsi des actes accomplis par les êtres vivants une série de miracles. Certes, Magendie n'était pas homme à se laisser intimider par ce fantôme; mais, ou bien il simplifiait systématiquement et artificiellement les faits, pour ne les dominer que d'une manière incomplète, ou bien la multiplicité des conditions auxquelles obéissent les phénomènes vitaux lui enlevait toute confiance théorique en la conclusion. Or, sans conclusions point de science. Claude Bernard se montra donc, et cela, nous le répétons, presque dès ses débuts, supérieur à la fois à 
Magendie et à Bichat, puisqu'au sentiment de l’innombrable multiplicité des inconnues physiologiques il joignait celui de leur subordination aux lois générales de la matière, et par suite de leur obéissance aux appels de la méthode expérimentale.

La physiologie pouvait donc pousser ses racines dans le sol ferme où se sont implantées ses sœurs aînées, la physique et la chi. mie. Cependant la complexité des problèmes qu'elle comprend exigeait que les règles de la méthode expérimentale fussent exposées sous des formules spéciales, en vue des procédés intellectuels et manuels qui lui sont spécialement applicables. La réalisation de cette œuvre a préoccupé Claude Bernard pendant toute la première phase de sa vie scientifique. Mais l'entraînement du laboratoire, la chasse aux découvertes, absorbait tous ses instants, si bien qu'il ne pouvait démontrer la méthode 
qu'à la façon dont Diogène démontrait le mouvement.

Et jamais chasse aux découvertes ne fut plus fructueuse. En vingt ans, Claude Bernard a plus trouvé de faits dominateurs, non-seulement que les physiologistes français qui, peu nombreux, travaillaient à ses côtés, mais que l'ensemble des physiologistes du monde entier. L'action des diverses glandes digestives et notamment du pancréas, la glycogénie animale, la production expérimentale du diabète, l'existence des nerfs vaso-moteurs et la théorie de la chaleur animale, l'action des poisons étudiés en eux-mêmes et comme moyen d'analyse des phénomènes physiologiques, l’innombrable quantité de faits nouveaux, de déductions sagaces, d'aperçus ingénieux et suggestifs que contiennent non-seulement ses mémoires spéciaux, mais les quatorze volumes où, depuis ses Lecons de physiologie expérimentale appliquée à la 
médecine (183̆30\%6), jusqu’à ses Lecons sur le diabiète et la glycogénèse animale (1877), il rassemblait chaque année le résultat de ses recherches et le résumé de ses cours, lui avaient donné une situation de maitre, acceptée sans conteste en France et à l'étranger.

Il avait également, dans la hiérarchie officielle, atteint le premier rang. En 1854, une chaire de physiologie générale fut créée pour lui à la Sorbonne, chaire qu'avec un désintéressement et une délicatesse admirables il abandonna en 1868 à son élève M. Paul Bert; en 1855, il remplaça Magendie dans la chaire de médecine du Collège de France. Entré à l'Académie des sciences en 1854, il fut appelé en 1868 à remplacer Flourens à l'Académie française. Enfin, un décret de 1869 le fit entrer au Sénat : et il est à peu près le seul des membres de cette assemblée auquel jamais personne n'ait songé à faire reproche d'une 
nomination qui le surprit étrangement.

Quelques années avant que les honneurs inattendus de la littérature et de la poli-tique fussent ainsi venus le trouver dans son laboratoire, un événement considérable s'était passé dans sa vie. Une maladie longue et grave, pendant laquelle ses amis et lui désespérèrent de l'issue favorable, le condamna à l'inactivité physique. Il dut quitter son laboratoire, quitter Paris même, et redemander au pays natal, non en vain, la santé et la vie. Ces longs mois d'isolement et de repos rendirent à son esprit toute sa liberté. Pour la première fois, il eut le temps de méditer et de mettre en ordre, sur le papier, le résultat de ses réflexions solitaires. Une courte préface, déjà imprimée en épreuves, et qui devait précéder une sorte de traité de physiologie opératoire qui reste encore en préparation, s'agrandit par des additions successives, prit les dimensions d'une brochure, puis 
d'un livre, qui vit le jour en 1865. L'Introduction à l'Étude de la médecine expérimentale frappa d'étonnement et d'admiration les esprits cultivés. Les physiologistes y trouvèrent avec bonheur, réduites en formules précises, ordonnées avec un art merveilleux, éclairées par des exemples qui étaient eux-mêmes comme autant d'expériences intellectuelles, les règles de la méthode expérimentale, surveillant, saisissant, maîtrisant, malgré ses efforts, le Protée organique aux métamorphoses trompeuses. Ceux que ne préoccupaient pas surtout les difficultés professionuelles furent frappés de la grandeur des problèmes étudiés, de la clarté de leur exposition, de l'aisance et de la bonne foi avec laquelle ils étaient ou résolus ou démontrés insolubles. Le style même en fut fort remarqué; sa saveur originale mit en goût jusqu'à l'Académie française : “Vous avez créé un style, » dit dans son discours de réception 
le sévère M. Patin. Et c'était vrai. Mais combien eût été étonné le vénérable critique s'il avait lu ces livres antérieurs oì Claude Bernard se contentait d'énumérer', dans une narration souvent peu ordonnée, ses impressions de laboratoire! Chez ce maître éminent et naif, qu'aucune préoccupation de mise en scène ne hanta jamais, le style parlé ou écrit valait ce que valait l’idée. Dans la narration épisodique, on le trouve souvent traînant et confus; mais qu'un problème difficile se pose, que la pensée soit furcée de se replier comme pour vaincre un obstacle ou prendre un élan, alors il se serre, s'épure, s'accentue en formules précises, souvent en paroles imagées.

Tel il était dans ses livres, tel Claude Bernard dans ses cours, dans ses conversations. Sa pensée n'était point docile à parler toutes les langues et jouer tous les rôles; et jamais il ne fit rien pour la disci- 
pliner à quelque convention d'habitudes sociales ou de métier. Que si elle s'échappait, il la suivait sans révolte, laissant là le discours languissant, la leçon confuse, et ne prêtant plus l'oreille qu'à ce qu'elle lui disait tout bas; mais si elle s'intéressait à la chose actuelle, alors ce professeur ou ce causeur, tout à l'heure pénible et diffus, se réveillait vivant, ingénieux, clair, éloquent, avec des mouvements surprenants et sondains, et toujours avec les deux qualités du vrai génie, l'aisance et la bonne foi.

Et nul ue les posséda à un plus hant degré. Cette aisance à s'élever sur les hauts sommets, à se mouvoir parmi les difficultés les plus ardues, a frappé surtout les lecteurs de ses admirables articles de la Revue des Deux-Mondes. On pouvait dire de lui ce que le poëte disait de la déesse : incessu patuit. Un homme éminent, an sortir de ces lectures, me disait un jour: " Il ne me fait pas seulement croire que je comprends, 
comme vous faites tous; il me fait réellement comprendre. » Et, de fait, il avait compris. Cette aisance, il l'importait de ses habitudes physiologistes dans le domaine philosophique. Nul ne fit jamais plus simplement, plus naïvement une découverte. Dans cette phase première de la chasse aux idées, comme disait Helvétius, qui consiste à voir et lever le gibier, il apportait une sûreté de vue, une perspicacité étonnante. La plupart des chercheurs scientifiques sont des espèces de somnambules qui ne voient que ce qu'ils cherchent, que ce qui est sur la trace de leurs idées; leur œil est fixé sur un point, et non-seulerrent ils ne perçoivent pas ce qui passe à côté de ce point, mais même ce qui s'y présente sans avoir été prévu. Claude Bernard semblait, suivant l'expression d'un de ses élèves, avoir des yeux tout autour de la tête, et c'était avec stupéfaction qu'on le voyait, au cours d'une expérience, signaler des phénomènes évi- 
dents, mais que personne, hormis lui, n'avait aperçus. Il découvrait comme les autres respirent.

Avec l'aisance, la bonne foi. Ce fut sa qualité maîtresse. Jamais il ne se départit de la sincérité profonde de l'homme de science, qui doit chercher la vérité pour elle et pour les vérités qui la suivent, sans s'inquiéter jamais des conséquences lointaines ou indirectes qu'en voudront tirer ceux qui, semblables à des avocats, ont une cause à défendre. Nul ne fut plus passif daus la déduction, et ne l'exprima avec une siricérité plus candide. De là vient que ses écrits peuvent et ont pu servir, à tour de rôle, à tous les souteneurs de thèses. Que s’il expose le déterminisme cérébral des actes intellectuels, les matérialistes le compteront parmi les leurs; que s'il déclare qu'entre la pensée et le cerveau il y a le même rapport qu'entre l'heure et l'horloge, les spiritualistes le voudront enrôler. En 
réalité, il n'est que physiologiste, livrant des faits nouveaux qui viennent rajeunir l'éternelle dispute des spéculateurs.

C'est cette admirable bonne foi, qui, dans le domaine restreint de la physiologie et de la médecine, explique l'apparente contradiction entre sa foi scientifique et son incrédulité pratique. Il ent toujours au plus haut degré ce double sentiment, que la physiologie sera la base nécessaire d'une médecine sûre d'elle-même, et que la physiologie actuelle est encore bien éloignée de fournir quelque certitude pratique. Ses propres déconvertes, il en sentait toute l'importance comme fondements de l'édifice médical, mais il ne partageait pas les illusious de ceux qui, avec un empressement dont il a bien souvent souri, les transportaient dans le domaine des applications cliniques ou thérapeutiques. Ce sentiment des distances, qui eût découragé de moins vaillants, ne l'émouvait nullement, et il n'avait pas be- 
soin, pour être fort et persévérant, de l'enivrement des illusions. Aussi, lui qui enseignait que la médecine est ou doit être une science, se montrait-il fort sceptique au regard des médecins, et, quand il en parlait, il semblait toujours que l'ombre de Sganarelle passât devant lui.

L'Introduction à l'Étude de la médecine expérimentale marque dans la vie de Claude Bernard une phase nouvelle. De là datent ces écrits philosophiques qui lui ont fait ouvrir les portes de l'Académie française. De là, des livres (Recherches sur les propriétés des tissus vivants, Lecons de pathologie expérimentale, etc.) où le groupement des faits prend le pas sur les constatations de détail, et où il s'efforce, reprenant en sons-œurre ses découvertes anciennes, d'en amener l'étude à toute la précision et la perfection que peuvent comporter les moyens d'action de la science actuelle.

Ce n'est pas à dire qu'il s'écartât complé- 
tement de ces régions de l'inconnu où il avait fait jadis de si riches moissons. Ses derniers travaux sur l'identité fondamentale des propriétés de tissu et des fonctions élémentaires dans le règne animal et le règne végétal, sur l'anesthésie par le chloroforme ou l'éther des végétaux inférieurs, et par suite sur la généralité d'action des substances toxiques, montrent que l'esprit créateur était vivant en lui.

De nouvelles découvertes devaient, cette année, fournir une preuve nouvelle de sa fécondité agissante. Ses amis, ses élèves en ont reçu la confidence incomplète, et il résulte des quelques paroles qui lui sout échappées que la théorie des fermentations allait recevoir de ces recherches, exécutées pendant les vacances dernières, des clartés inattendues. Ce travail considérable, dont, il y a quatre jours, il disait encore : "C'est dommage, c'eût été bien finir », est perdu pour la science. 
Le 31 décembre, le froid le saisit dans le Jaboratoire du Collége de France; bientôt survinrent les frissons, la fièvre et les phénomènes spéciaux, signes d'une inflammation rénale. Rien ne put enrayer la marche d'un mal dont il suivait tous les progrès. Sans illusion sur la fatalité de la catastrophe, il l'envisageait d'un œil calme, se refusant avec un sourire aux pieux mensonges de sa famille scientifique. Il était de ceux dont le regard ne s'effraye pas de l'inconnu.

Les sentiments personnels doivent se taire dans cet immense deuil de la science. Et cependant, ce n'est pas seulement la perte d'un grand homme qui mouille les yeux de ceux qui entourent son cercueil : tant de bienveillance, de simplesse d'âme, de générosité naïve étaient unies à ce génie! Il en est dont la main tremble en essayant d'esquisser quelques traits de ce noble et grand caractère.

Rien dans cette vie si pure, si harmoni- 
que, n’a été détourné du but principal. Épris de littérature, d'art et de philosophie, Claude Bernard n'a rien perdu comme physiologiste à ces nobles passions : toutes, au contraire, lui ont servi dans le développement de la science avec laquelle il s'était identifié, et dont il reste l'expression la plus complète et la plus élevée. Il fut physiologiste comme nul ne l'avait été : “Claude Bernard, disait un savant étranger, n'est seulement point un physiologiste, c'est la physiologie. )

Sa mort elle-même semble marquer pour la science une ère nouvelle. Pour la première fois dans notre pays, un homme de science va recevoir les honneurs publics, réservés jusqu’ici aux illustrations politiques ou guerrières. Le gouvernement s'est honoré hier en demandant aux Chambres, qui l'ont accordé à l'unanimité, de faire aux frais de l'État des funérailles solennelles au maître qui n'est plus. Et le mot de 
M. Gambetta, parlant au nom de la commi:sion du budget, résume tout ce que nous avons dit: "La lumière qui vient de s'éteindre ne sera pas remplacée. »

\section{Paul Bert.}

Paris, le 12 février 1878. 



\section{LA SGIENGE EXPÉRIMENTALE}

\section{DU PROGRĖS}

\section{DANS LES SCIENCES PHYSIOLOGIOUES}

La méthode expérimentale, qui depuis longtemps est appliquée avec tant de succès à l'étude des phénomènes des corps bruts, tend de plus en plus aujourd'hui à s'introduire' dans l'étude des phénomènes des êtres vivants; mais beaucoup de savants doutent encore de son utilité réelle, et il en est qui croient que la spontanéité vitale sera toujours un obstacle insurmontable à l'application d'une méthode commune d'investigation dans les sciences physiologiques et dans les sciences physico-chimiques. 
Les corps bruts étant tous depourvus de sponlanéité, les manifestations de leurs propriétés demeurent enchaînées d'une manière absolue aux variations des circonstances qui les environnent, ce qui permet à l'expérimentateur de les atteindre facilement et de les modifier à son gré.

l.es êtres vivants, étant au contraire doués de spontanéité, nous apparaissent comme s'ils étaient tous pourvus d'une force intérieure qui rend les manifestations de la vie d'autant plus indépendantes des variations des influences extérieures que l'être s'élève davantage dans l'échelle de l'organisation. Chez l'homme et chez les animaux supérieurs, cette force vitale semble avoir pour résultat de soustraire le corps vivant aux influences physico-chimiques générales et de le rendre ainsi tout à fait inaccessible aux procédés ordinaires d'expérimentation. J'un autre côté, tous les phénomènes des animaux vivants sont reliés par la sensibilité et maintenus par elle dans une harmonie réciproque telle qu'il paraît impossible de séparer une partie de leur organisme sans amener immédiatement un trouble dans tout son ensemble. 
DANS LES SCIENGES PHYSIOLOGIQUES.

Beaucoup de médecins et de naturalistes on exploité ces divers arguments pour s'élever contre l'emploi de l'expérimentation chez les êtres vivants. Ils ont admis que la force vitale était en opposition avec les forces physico-chimiques, qu'elle dominait tous les phénomènes de la vie, les assujettissait à des lois tout à fail spéciales, et faisait de l'organisme un tout vivant auquel l'expérimentateur ne pourait toucher sans détruire le caractère de la vie même. Cuvier, qui a partagé cette opinion, et qui pensait que la physiologie devait être une science d'observation et de déduction anatomique, s'exprime ainsi : "Toutes les parties d'un corps vivant sont liées; elles ne peuvent agir qu'autant qu'elles agissent toutes ensemble. Vouloir en séparer une de la masse, c'est la reporter dans l'ordre des substances mortes, c'est en changer entièrement l'essence ${ }^{1}$. »

Si les objections précédentes étaient fondées, il faudrait reconnaître, ou bien qu'il n'y a pas de déterminisme possible dans les phénomènes de

1. Lettre de Cuvier à J.-C. Mertrud, Lecons d'anatomie comparée, p. 5. Paris, an vill. 
la vie, ce qui serait nier purement et simplement la physiologie expérimentale, ou bien il faudrait admettre que la force vitale doit être étudiée suivant une méthode particulière, et que la science des corps vivants doit reposer sur d'autres principes que la science des corps inertes.

Ces idées, qui ont été florissantes à d'autres époques, s'évanouissent aujourd'hui de plus en plus sous l'influence des progrès de la physiologie. Cependant il importe d'en extirper les derniers germes, parce que ce qui reste encore de ces idées dans certains esprits constitue un véritable obstacle à la marche de la science physiologique et de la médecine expérimentale. Je me propose de démontrer que les phénomènes des corps vivants sunt, comme ceux des corps bruts, soumis à un déterminisme absolu et nécessaire. La science vitale ne peut employer d'autres méthodes ni avoir d'autres bases que celles de la science minérale, et il n'y a aucune différence à établir entre les principes des sciences physiologiques et ceux des sciences physico-chimiques. 


\section{I}

La spontanéité dont jouissent les êtres vivants n'empêche pas le physiologiste de leur appliquer la méthode expérimentale '. En effet, malgré cette spontanéité, les êtres vivants ne sont pas indépendants des influences du monde extérieur, et leurs fonctions sont constamment lićes à des conditions qui en règlent l'apparition d'une manière déterminée et nécessaire.

Dès qu'on entre dans l'étude des mécanismes propres aux phénomènes de la vie, on s'apelcoit bientôt que la spontanéité apparente dont jouissent les corps vivants n'est que la conséquence toute naturelle de certaines circonstances bien déterminées, et il nous sera facile de prou-

1. Je renvoie le lecteur, pour la démonstration technique de ces considérations, à mon ouvrage : Introduction ¿̀ l'étude de la médecine expérimentale. Paris, 1865. 
ver qu'au fond les manifestations des corps vivants, aussi bien que celles des corps bruts, sont rattachées à des conditions d'ordre purement physico-chimique. Nous ajouterons que le problème que se posent le physiologiste et le médecin expérimentateur n'est point de remonter à la cause première de la vie, mais seulement d'arriver à la connaissance de ces conditions physico-chimiques déterminantes de l'activité vitale.

Notons d'abord que l'indépendance de l'être vivant dans le milieu cosmique ambiant n'apparaît que dans les organismes complets et élevés.

Dans les êtres inférieurs réduits à un organisme élémentaire, tels que les infusoires, il n'y pas d'indépendance réelle. Ces êtres ne manifestent les propriétés vitales, souvent très-actives, dont ils sont doués que sous l'influence de l'humidité, de la lumière, de la chaleur extérieure, et dès qu une ou plusieurs de ces conditions viennent à manquer, la manifestation vitale cesse, parce que les phénomènes physicochimiques qui lui sont parallèles s'arrêtent. 
DANS LES SCIENCES PHYSIOLOGIQUES.

Beaucoup de ces animaux tombent alors dans un état de vie latente qui n'est autre chose qu'un état d'indifférence chimique du corps organisé vis-à-vis du monde extérieur. Cette suspension complète des manifestations apparentes de la vie est susceptible de durer un temps en quelque sorte indéfini. Spallanzani a vu la vitalité reparaître sous l'influence d'une goutte d'eau chez des anguillules du blé niellé, inertes et desséchées depuis près de trente ans ${ }^{1}$. Dans ce cas l'eau, restituée au corps, y a simplement fait reparaître les phénomènes chimiques, et a permis aux tissus de manifester leurs propriétés vitales.

Dans les végétaux, les phénomènes de la vie sont également liés quant à leurs manifestations aux conditions de chaleur, d'humidité et de lumière du milieu ambiant, et c'est ce qui constitue l'influence des saisons, que tout le monde connaît.

1. Spallanzani, Observations et expériences sur quelques animaux surprenans que l'observateur peut à son gré faire passer de la mort ì la vie. Euvres, in-80, p. 203. 
Il en est de même pour les animaux à sang froid; les phénomènes de la vie s'engourdissent ou se réveillent chez eux, suivant les mêmes conditions climatériques de chaleur, de froid, d'humidité, de sécheresse.

Or l'eau, la chaleur, l'électricité, sont aussi les excitants des phénomènes physico-chimiques, de telle sorte que les influences qui provoquent accélèrent ou ralentissent les manifestations vitales ch€z les êtres vivants sont exactement $l$ is mêmes que celles qui provoquent, accélèrent ou ralentissent les manifestations minérales dians les corps bruts.

Loin de voir, à l'exemple des vitalistes, une sorte d'opposition ou d'incompatibilité entre les conditions des fonctions vitales et les conditions des actions minérales, il faut au contraire constater entre ces deux ordres de phénomènes un parallélisme complet et una relation directe et nécessaire.

Cette relation est plus étroite chez les êtres inférieurs, chez les végétaux et chez les animaux à sang froid; mais chez l'homme et chez les autres animaux à sang chaud il y a en gé- 
DANS LES SCIENCES PHYSIOLOGIQUES.

néral une indépendance évidente entre les fonctions de l'organisme et les conditions du milieu ambiunt. Les phénomènes vitaux ne subissent plus dans leurs manifestations l'influence des alternatives des saisons ni celle des variations cosmiques. Par suite d'un mécanisme protecteur plus complet, l'animal possède et maintient en lui, dans un milieu intérieur qui lui est propre, les conditions d'humidité et de chaleur nécessaires aux manifestations des phénomènes vitaux. L'organisme de l'animal à sang chaud étant suffisamment protégé n'entre que trèsdifficilement en équilibre avec le milieu extérieur : il garde en quelque sorte ses organes en serre chaude, il leur conserve ainsi leur activité vitale. C'est de même que nous voyons, dans les serres de nos jardins, se manifester une activité vitale végétative indépendante des chaleurs et des frimas extérieurs, mais liée cependant d'une manière intime et nécessaire aux conditions physico-chimiques de l'atmosphère intérieure de la serre.

Les manifestations de la vie que nous observons chez l'homme ou chez un animal supé- 
rieur sont beaucoup plus complexes qu'elles ne nous apparaissent; mais ce qu'il ne faut jamais oublier, c'est que, quelle qu'en soit la complexité, elles sont toujours la résultante des propriétés intimes d'une foule d'éléments organiques dont l'activité est liée aux conditions physico-chimiques des milieux internes où ils sont plongés. Nous supprimons dans nos explications le inilieu intérieur que nous ne voyons pas pour ne considérer que le milieu extérieur qui est sous nos yeux, et c'est ainsi que nous pouvons croire faussement qu'il y a dans l'être vivant une force vitale qui viole les lois physico. chimiques du milieu cosmique général.

Les machines vivantes sont donc créées et construites de telle facon qu'en se perfectionnant elles deviennent de plus en plus libres dans le monde extérieur; mais il n'en existe pas moins la détermination vitale dans leur milieu interne, qui par suite de ce même perfectionnement s'est isolé de plus en plus dı milieu cosmique général. Les machines que l'intelligence de l'homme crée, quoique infiniment plus grossières; possèdent aussi une in- 
DANS LES SGIENCES PHYSIOLOGIOUES.

dépendance qui n'est que l'expression du jeu de leur mécanisme intérieur. Une machine à vapeur possède une activité indépendante des conditions physico-chimiques du milieu extérieur, puisque, par le froid, le chaud, le sec et l'humide, la machine continue à marcher'; mais pour le physicien qui descend dans le milieu intérieur de la machine, il trouve que cette indépendance n'est qu'apparente, et que l̉e mouvement de chaque rouage intérieur est déterminé par des conditions physiques absolues et dont il connaît la loi. De même pour le physiologiste, s'il peut descendre dans le milieu intérieur de la machine vivante, il y trouvera un déterminisme qui doit devenir pour lui la base réelle de la science expérimentale des corps vivants.

Pour comprendre l'expérimentation sur les êtres vivants d'une organisation élevée, il faut nécessairement tenir compte de deux milieux : le milieu cosmique ou extra-organique, qui est commun aux êtres vivants et aux corps bruts, et le milieu intra-organique, qui est spécial anx êtres virants. Ce dernier milieu, qui est en rap- 
port avec nos éléments organiques actifs, mascles, nerfs, glandes, etc., est formé par tous les liquides intra-organiques et blastématiques ${ }^{1}$. Nous trouvons dans ce milieu liquide les con:ditions de température, l'air et les aliments dissous dans l'eau, car, ainsi que nous l'avons dit ailleurs ${ }^{2}$, tous les éléments organiques actifs qui composent notre organisme sont nécessairement aquatiques, et ce n'est que par un artifice de construction que notre corps peut exister et se mouvoir dans l'air sec.

La médecine expérimentale ou scientifique sera surtout fondée sur la connaissance des pro. priétés du milieu intra-organique.

Quand un médicament exerce sur nous son action, ce n'est point dans notre estomac qu'il agit, mais seulement dans notre milieu intra-

1. Voyez Claude Bernard, Lecons de physiologie expe rimentale appliquée à la médecine. Paris, 1855-56, 2 vol. - Lecons sur la physiologie et la pathologie du système nerveux. Paris, 1858, 2 vol. - Lecons sur les propriétés physiologiques des liquides de l'organisme. Paris, 1859, 2 vol.

2. Voy. Etude sur la physiologie du cœur, p. 316. 
organique, après avoir pénétré dans notre sang et s'être mis en contact avec nos particules or'ganisées. Cette idée du milieu intérieur, dirigeant mes études en physiologie, m'a servi à déterminer d'une manıère plus précise l'action des substances toxiques sur les divers éléments de notre $\operatorname{corps}^{1}$; mais en outre il en résulte des considérations nouvelles, qui sont destinées à guider le physiologiste dans ses expérimentations et à servir de base à la fois à la physiologie et à la pathologie générales. En effet, au point de vue médical ou thérapeutique, nous ne saurions trouver, ni chez l'homme ni chez les animaux élevés, une indépendance vitale à l'égard des poisons et des médicaments. Tous les jours nous pouvons modifier les phénomènes de la vie ou les éteindre en faisant pénétrer des substances actives dans notre sang ou dans notre milieu organique; mais ce serait une illusion que de ne voir, dans toutes ces modifications si variées et si multiples de l'organisme,

1. Voy. Etudes physiologiques sur quelques poisons américains, le curare, p. 237. 
que l'expression indéterminée d'une force vitale quelconque ${ }^{1}$. Elles dépendent toutes au contraire de conditions physico-chimiques précises survenues dans notre milieu intérieur ou dans les les éléments histologiques de nos tissus.

Autrefois Buffon avait cru qu'il devait exister dans le corps des êtres vivants un élément organique particulier qui ne se retrouverait pas dans les corps minéraux ${ }^{2}$. Les progrès des sciences chimiques ont détruit cette hypothèse en montrant que le corps vivant est exclusivement constitué par des matières simples ou élé mentaires empruntées au monde minéral.

On a pu croire de même à l'activité d'une force spéciale pour la manifestation des phénomènes de la vie; mais les progrès des sciences physiologiques détruisent également cette se conde hypothèse, en faisant voir que les propriétés vitales n'ont pas plus de spontanéité par elles-mêmes que les propriétés minérales,

1. Claude Bernard, Lecons sur les effets des substances toxiques et médicamenteuses. Paris, 1857.

2. Buffon, Euvres cumplètes, publiées par Lacépède, t. IX, p. 25 . 
DANS LES SCIENCES PHYSIOLOGIOUES.

et que ce sont les mêmes conditions physicochimiques générales qui président aux manifestations des unes et des autres.

On ne saurait inférer de ce qui vient d'être dit que nous assimilons les corps vivants aux corps bruts; le bon sens de tous protesterait immédiatement contre une pareille confusion. Il est évident que les corps vivants ne se comportent pas comme les corps inanimés. Il s'agit seulement de bien caractériser et de bien définir leur différence, car c'est un point capital pour bien comprendre la science physiologique expérimentale.

De toutes les définitions de la vie, celle qui est à la fois la moins compromettante et la plus vraie est celle qui a été donnée par l'Encyclopédie: "la vie est le contraire de la mort. " Cette définition est d'une clarté naïve, et cependant nous ne pourrons jamais rien dire de mieux, parce que nous ne saurons jamais ce qu'est la vie en elle-même. Pour nous, un corps n'est vivant que parce qu'il meurt et parce qu’il est organisé de manière à ce que, par le jeu naturel de ses fonctions, il entretient son 
organisation pendant un certain temps et se perpétue ensuite par la formation d'individus semblables à lui. La vie a done son essence dans la force ou plutôt dans l'idée directrice du développement organique; c'est la force vitale ainsi comprise qui constituait la force médicatrice d'Hippocrate, la force séminale et l'archeus faber de Van Helmont.

Si je devais définir la vie d'un seul mot, je dirais : la vie, c'est la création. En effet, la vie pour le physiologiste ne saurait être autre chose que la cause première créatrice de l'orgarisme qui nous échappera toujours, comme toutes les causes premières. Cette cause se manifeste par l'organisation; pendant toute sa durée, l'être vivant reste sous l'empire de cette influence vitale créatrice, et la mort naturelle arrive lorsque la création organique ne peut plus se réaliser.

L'esprit de l'homme ne peut concevoir un effet sans cause, la vue d'un phénomène éveille toujours en lui une idée de causalité, et toute la science humaine consiste à remonter des effets ob́servés à leur cause; mais de tout temps 
DANS LES SGIENGES PHYSIOLOGIQUES. 53

les philosophes et les savants ont distingué deux ordres de causes, les causes premières et les causes secondes ou prochaines. - Les causes premières, qui sont relatives à l'origine des choses, nous sont absolument impénétrables; les causes prochaines, qui sont relatives aux conditions de manifestation des phénomènes sont à notre portée et peuvent nous être connues expérimentalement. Newton a dit que celui qui se livre à la recherche des causes premières donne par cela même la preuve qu'il n'est pas un savant. En effet, cette recherche reste stérile, parce qu'elle nous pose des problèmes qui sont inabordables à l'aide de la méthode expérimentale.

En résumé, il y a dans un phénomène vital, comme dans tout autre phénomène naturel, deux ordres de causes : d'abord une cause première, créatrice, législative et directrice de la vie, et inaccessible à nos connaissances, ensuile une cause prochaine ou exécutive du phénomène vital, qui toujours est de nature physico-chimique, et tombe dans le domaine de l'expérimentateur. La cause première de la 
vie donne l'évolution ou la créalion de la machine organisée; mais la machine, une fois créée, fonctionne en vertu des propriétés de ses éléments constituants et sous l'influence des conditions physico-chimiques qui agissent sur eux. Pour le physiologiste et le médecin expérimentateur, l'organisme vivant n'est qu'une machine admirable, douée des propriétés les plus merveilleuses, mise en action à l'aide des mécanismes les plus complexes et les plus délicats. C'est une machine dont ils doivent analyser et déterminer le mécanisme, afin de pouvoir le modifier, car la mort accidentelle n'est que la dislocation ou la destruction de l'organisme par suite de la rupture ou de la cessation d'action d'un ou de plusieurs de ces mécanismes vitaux.

\section{II}

La recherche des causes premières, avonsnous dit, n'est point du domaine scientifique. 
DANS LES SGIENGES PHYSIOLOGIQUES.

Quand l'expérimentateur est parvenu au déterminisme des phénomènes, il ne lui est pas donné d'aller au delà, et sous ce rapport la limite de sa connaissance est la même dans les sciences des corps vivants et dans les sciences des corps bruts.

Le nature de notre esprit nous porte d'abord à rechercher la cause première, c'est-à-dire l'essence ou le pourquoi des choses. En cela, nous visons plus loin que le but qu'il nous est donné d'atteindre, car l'expérience nous apprend bientôt que nous ne pouvons pas aller au delà du comment, c'est-à-dire au delà du déterminisme qui donne la cause prochaine ou la condition d'existence des phénomènes.

Ce que nous appelons le déterminisme d'un phénomène n'est rien autre chose que la cause déterminante ou la cause prochaine, c'est-ì-dire la circonstance qui détermine l'apparition du phénomène et constitue sa condition ou l'une de ses conditions d'existence. Le mot déterminisme a une signification tout à fait différente de celle du mot fatalisme. Le fatalisme suppose la manifestation nécessaire d'un phénomène 
indépendamment de ses conditions, tandis que le déterminisme n'est que la condition nécessaire d'un phénomène dont la manifestation n'est pas forcée. Le fatalisme est donc antiscientifique à l'égal de l'indéterminisme.

Lorsque, par une analyse expérimentale successive, nous avons trouvé la, cause prochaine ou la condition élémentaire d'un phénomène, nous avons atteint le but scientifique que nous ne pourrons jamais dépasser.

Quand nous savons que l'eau avec toutes ses propriétés résulte de la combinaison de l'oxygène et de l'hydrogène dans certaines proportions, et que nous connaissons la condition de cette combinaison, nous savons tout ce que nous pouvons savoir scientifiquement à ce sujet; mais cela répond au comment et non au pourquoi des choses. Nous savons comment l'eau peut se faire; mais pourquoi la combinaison d'un volume d'oxygène et de deux volumes d'hydrogène donne-t-elle de l'eau, nous n'en savons rien, nous ne pouvons pas le savoir, et nous ne devons pas le chercher.

En médecine aussi bien qu'en chimie, il 
DANS LES SCIENGES PHYSIOLUGIQJES.

n'est pas scientifique de poser la question du pourquoi : cela ne peut en effet que nous égarer dans des questions insolubles et sans applications. Serait-ce pour se moquer de cette tendance antiscientifique de la médecine qui résulte de l'absence du sentiment de cette limite de nos connaissances que Molière a mis dans la bouche de son candidat docteur, à qui l'on demandait pourquoi l'opium fait dormir, la réponse suivante: Quia est in eo virtus dormitiva, cujus est natura sensus assoupire? Cette réponse paraît plaisante ou absurde; elle est cependant la seule qu'on pourrait faire.

De même, si l'on voulait répondre à cette question : "Pourquoi l'hydrogène, en se combinant avec de l'oxygène, fait-il de l'eau? » on serait obligé de dire : « Parce qu'il y a dans l'hydrogène une propriété capable d'engendrer l'eau. »

C'est done seulement ]a question du pourquoi qui est absurde, puisqu'elle entraîne une réponse qui paraît naïve ou ridicule. Il vaut mieux reconnaître que nous ne savons pas, et que c'est là que se place la limite de notre 
connaissance. Nous pouvons savoir comment et dans quelles conditions l'opium fait dormir; mais nous ne saurons jamais pourquoi.

Les propriétés de la matière vivante ne peuvent être manifestées et connues que par leurs rapports avec les propriétés de la matière brute, d'où il résulte que les sciences physiologiques expérimentales ont pour base nécessaire les sciences physico-chimiques, auxquelles elles empruntent leurs procédés d'investigation et leurs moyens d'action. Le corps vivant est pourvu sans doute de propriétés et de facultés tout à fait spéciales à sa nature, telles que la plasticité organique, la contractilité, la sensibilité, l'intelligence; néanmoins toutes ces propriétés et toutes ces facultés sans exception, de quelque ordre qu'elles soient, trouvent leur déterminisme, c'est-à-dire leurs moyens de manifestations et d'action, dans les conditions physico-chimiques des milieux extérieur et intérieur de l'organisme. Mais dans les phénomènes vitaux pas plus que dans les phénomènes minéraux la condition d'existence d'un phénomène ne saurait rien nous apprendre sur sa nature. 
DANS LES SCIENCES PHYSIOLOGIOUES. 59

Quand nous savons que l'excitation extérieure de certains nerfs et que le contact physique et chimique du sang, à une certaine température, avec les éléments nerveux cérébraux sont nécessaires pour manifester la pensée ainsi que les phénomènes nerveux et intellectuels, cela nous indique le déterminisme ou les conditions d'existence de ces phénomènes, mais cela ne saurait rien nous apprendre sur la nature première de l'intelligence. De même, quand nous savons que le frottement et les actions chimiques développent l'électricité, cela nous indique le déterminisme ou les conditions du phénomène, mais cela ne nous apprend rien sur la nature première de l'électricité.

L'expérimentateur peut modifier tous les phénomènes de la nature qui sont à sa portée.

Par une disposition que nous devons sans doute trouver fort sage, il ne pourra jamais agir sur les corps célestes; c'est pourquoi l'astronomie est condamnée à rester à tout jamais une science d'observation pure. "Sur la terre, dit Laplace, nous faisons varier les phénomènes par des expériences; dans le ciel, nous obser- 
vons avec soin tous ceux que nous offrent les mouvements célestes ${ }^{1}$. »

Parmi les sciences des phénomènes terrestres qui seules sont appelées à être des sciences d'expérimentation, les sciences minérales ont été les premières, à cause de la plus grande simplicité de leurs phénomènes, à devenir accessibles à l'expérimentateur'; mais c'est à tort qu'on a voulu exclure l'expérimentation de la science des êtres vivants, en disant que l'organisme s'isole comme un petit monde (microcosme) dans le grand nombre (macrocosme), et que sa vie représente la résultante d'un tout ou d'un système invisible dont nous ne pouvons qu'observer les effets sans les modifier.

Si la médecine, par exemple, voulait rester une science d'observation, le médecin devrait se contenter d'observer ses malades, se burner à prédire la marche et l'issue de leurs maladies, mais sans y toucher plus que l'astronome ne touche à ses planètes. Donc le médecin expérimente dès qu'il donne un remède actif, car c'est 
DANS LES SCIENCES PHYSIOLOGIQUES.

une véritable expérience qu'il fait en essayant d'apporter une modification quelconque dans les symptômes de la maladie. L'expérimentation scientifique doit ètre fondée sur la connaissance du déterminisme des phénomènes, autrement l'expérimentation n'est encore qu'aveugle et empirique. L'empirisme doit être subi comme une période nécessaire de l'évolution de la médecine expérimentale; mais il ne saurait être érigé en système, comme l'ont voulu quelques médecins.

L'expérimentation peut être appliquée à tous les phénomènes naturels de quelque ordre qu'ils soient, et cela se comprend, puisque l'expérimentateur n'engendre pas les phénomènes, mais agit seulement et exclusivement sur leur état antérieur, c'est-à-dire sur la condition physicochimique qui en procède et en détermine immédiatement la manifestation.

Quand l'expérimentateur refroidit un corps liquide pour le faire cristalliser, il n'agit pas sur la cristallisation, qui est la propriété innée de la matière minérale, il ne fait que déterminer la condition dans jaquelle elle a lieu. 
Quand on chauffe à 100 degrés du chlorure d'azote et qu'il s'ensuit une explosion qui devient à la fois une source puissante de mouvement et de chaleur, on n'agit pas sur l'explosion elle-même, on ne fait qu'apporter une température de 100 degrés qui est la condition déterminante de l'explosion.

Pour les phénomènes organiques, il en est absolument de même.

Quand on a mis par exemple des globules de levûre de bière dans un liquide sucré, qu'on maintient à une température inférieure à 10 degrés, rien ne se passe dans le liquide; la levîre engourdie reste sans action sur le sucre, et il ne se forme ni acide carbonique ni alcool: mais si on élève la température à +30 degrés, on voit bientôt la fermentation marcher avec une très-grande activité. Dans ce cas encore, on n'a pas agi sur la propriété de fermentation qui est essentielle el innée à la Jevûre, on n'a fait que produire les conditions chimico-physiques sous l'influence desquelles la fermentation s'arrète ou se manifeste.

Si maintenant nous prenons nos exemples 
DANS LES SCIENCES PHYSIOLOGIQUES.

dans les phénomènes les plus élevés et les plus mystérieux des êtres vivants, nous verrons que l'application de l'expérimentation doit toujours ètre comprise de la même manière.

Ce qui se passe chaque jour sous nos yeux pendant l'incubation dans l'œuf d'une poule serait bien fait pour nous émerveiller et pour nous montrer toute la profondeur de notre ignorance; mais par habitude nous cessons de nous étonner des phénomènes vulgaires, parce que nous cessons d'y réfléchir.

On a comparé l'évolution organique silencieuse qui s'accomplit dans cet œuf à l'harmonie d'un corps céleste dans l'espace. Van Helmont, qui nous apparait comme une sorte d'esprit lucide au milieu des ténèbres du moyen âge, avait placé dans l'œuf un archeus faber, ou une illée, qui dirigeait l'évolution ${ }^{1}$. Cela ressemble bien en effet à une idée qui se développe, car dès ce moment tout est coordonné, tout est prévu non-seulement pour l'évolution du

1. Voyez J. Guislain, thèse sur Van IIelmont, la Nature, etc., p. 164. 
nouvel être, mais pour son entretien fonctionnel durant sa vie entière, car la nutrition n'est que la génération continuée.

Et si maintenant nous recourons à la science moderne, nous verrons que dans l'œuf la partie essentielle se réduit à une petite vésicule ou cellule microscopique, tout le reste de l'œuf de l'oiseau, le jaune el le blanc, n'étant que des matériaux nutritifs destinés à fournir au développement qui doit se faire en dehors du corps maternel. Nous serions donc obligés de mettre dans la simple cellule organique microscopique qui compose l'œuf de tous les animaux une idée évolutive tellement complexe que non-seulement elle renferme tous les caractères spécifiques de l'être, mais qu'elle retrace encore tous les détails de l'individualité. C'est ainsi que chez l'homme une maladie qui apparaîtra par hérédité vingt ou trente ans plus tard se trouve déjà en germe dans cette vésicule mystérieuse.

Mais cette iảée spécifique contenue dans l'œuf ne se manifeste et ne se développe elle-même que sous l'influence de conditions purement physico-chimiques. Comme notre cellule de le- 
vûre de bière, la cellule de l'œuf reste engourdie au-dessous d'une certaine température, et ce n’est qu’à +35 degrés que l’idée organique manifestera son activité.

Je m'arrête ici : les exemples que j’ai cités, et qui se rapportent tous à des faits bien connus, me paraissent suffisants pour exprimer mon sentiment et faire comprendre ma pensée. L'expérimentateur ou le déterministe doit donc observer les phénomènes de la nature uniquement pour trouver leur cause déterminante, sans vouloir, pour les expliquer dans leurs causes premières, recourir à des systèmes qui peuvent flatter son orgueil, mais qui ne font en réalité que voiler son ignorance.

Il faut cesser, on le voit, d'établir entre les phénomènes des corps vivants et les phénomènes des corps bruts une différence fondée sur ce que l'on peut connaître la nature des premiers et que l'on doit ignorer celle des seconds.

Ce qui est vrai, c'est que la nature ou l'essence de tous les phénomènes, qu'ils soient vitaux ou minéraux, nous reste complétement 
inconnue. L'essence du phénomène minéral le plus simple est aussi totalement ignorée du chimiste et du physicien que l'est du physiologiste l'essence des phénomènes intellectuels ou la cause première d'un autre phénomène vital quelcon. que. Cela se conçoit d'ailleurs : la connaissance de la nature intime des choses ou la connaissance de l'absolu exigerait pour le phénomène le plus simple la connaissance de l'univers entier, car il est évident qu'un phénomène de l'univers est un rayonnement quelconque de cet univers, dans l'harmonie duquel il entre nécessairement pour sa part. La connaissance de l'absolu est donc la connaissance qui ne laisserait rien en dehors d'elle. L'homme y tend par sentiment, mais il est clair qu'il ne pourra la posséder tant qu'il ignorera quelque chose, et la raison paraît nous dire qu'il en sera toujours ainsi.

Toutefois la raison, même en servant de correctif au sentiment, ne le fait pas disparaître. L'homme, en se corrigeant, ne change pas sa nature pour cela; son sentiment, refoulé sur un point, reparaît et se fait jour ailleurs. C'est 
DANS LES SCIENCES PHYSIOLOGIQUES.

ainsi que l'expérience, qui vient à chaque pas montrer au savant que si connaissance est bornée, n’étouffe pas en lui son sentiment na.s turel, qui le porte à croire que la vérité absolue est de son domaine. L'homme se comporte intinctivement comme s'il devait y parvenir, et le pourquoi incessant qu'il adresse à la nature en est la preuve.

Il serait du reste mauvais pour la science que la raison ou l'expérience vînt étouffer complétement le sentiment ou l'aspiration vers l'absolu. Le savant dépasserait alors le but de la méthode expérimentale, comme celui qui, pour redresser une branche vers une meilleure direction, la romprait, et ferait cesser en elle toute séve et toute végétation. En effet, on le verra plus loin, c'est cette espérance de la vérité, constamment décue, constamment renaissante, qui soutient et soutiendra toujours les générations successives dans leur ardeur passionnée à étudier les phénomènes de la nature.

Le rôle particulier de la science expérimentale est de nous apprendre que nous ignorons, 
en nous montrant nettement que la limite de nos connaissances s'arrête au déterminisme; mais, par une merveilleuse compensation, à mesure que la science froisse notre sentiment et rabaisse notre orgueil, elle augmente notre puissance. Le savant qui a poussé l'analyse expérimentale jusqu'au déterminisme d'un phénomène voit clairement qu'il ignore ce phénomène dans sa cause première, mais il en est devenu maître; l'instrument qui agit lui reste inconnu dans son essence, mais il connaît la manière de s'en servir. Nous ignorons l'essence du feu, de l'électricité, de la lumière, et cependant nous en réglons les phénomènes à notre profit. Nous ignorons l'essence de la vie, mais nous n'en réglons pas moins les phénomènes vitaux dès que nous connaissons suffisamment leurs conditions d'existence. La seule différence est que dans les phénomènes vitaux le déterminisme est beaucoup plus difficile à atteindre, parce que les conditions sont infiniment plus complexes et plus délicates et qu'elles sont en outre combinées les unes avec les autres.

Le physicien et le chimiste, ne se placant pas 
D.ANS LES SGIENGES PHYSIOLOGIQUES.

en dehors de l'univers, peuvent étudier les corps et les phénomènes isolément, sans être obligés pouir les comprendre de les rapporter à l'ensemble de la nature; mais le physiologiste, se trouvant au contraire placé en dehors de l'organisme animal dont il peut voir l'ensemble, doit tenir compte de l'harmonie de cet ensemble en même temps qu'il cherche à pénétrer dans l'intérieur pour analyser le mécanisme de chacune des parties. Il s'ensuit que le physicien et le chimiste peuvent repousser toute idée de causes finales dans les faits qu'ils observent et que le physiologiste au contraire est porté à admettre une finalité harmonique et préétablie dans le corps organisé, dont toutes les actions particlles sont solidaires et génératrices les unes les autres.

Si, à l'aide de l'analyse expérimentale, on décompose l'organisme vivant en isolant ses diverses parties, ce n'est point pour les concevoir séparément. Quand on veut donner à la propriété physiologique d'un organe ou d'un tissu toute sa valeur et sa véritahle signification, il faut toujours le rapporter à l'organisme, 
et ne tirer de conclusion sur elle que relativement à ses effets dans l'ensemble organisé. Il faut reconnaître en un mot que le déterminisme dans les phénomènes de la vie est non-seulement un déterminisme très-complexe, mais que c'est en même temps un déterminisme harmoniquement subordonné. Les phénomènes physiologiques, si compliqués chez les animaux élevés, sont constitués par une série de phénomènes plus simples qui s'erigendrent les uns les autres en s'associant ou se continuant vers un but final commun.

Or l'objet essentiel pour le physiologiste est de déterminer par l'analyse expérimentale les conditions élémentaires des phénomènes physiologiques complexes et d'en saisir la subordination naturelle, afin d'en comprendre et d'en suivre les diverses combinaisons dans les mécanismes si variés que nous offrent les êtres vivants. L'emblème antique représenté par un serpent qui forme un cercle en se mordant la queue donne une image assez juste de la vie. En effet l'organisme vital forme un circuit fermé, mais ce cercle a une tête et une queue, 
D.ANS LES SCIENCES PHYSIOLOGIQUES. 71

en ce sens que tous les phénomènes vitaux n'ont pas la même importance, quoiqu'ils soient connexes et se fassent suite dans l'accomplissement du circulus vital. Ainsi les organes musculaires et nerveux entretiennent l'activité des organes qui préparent Je sang ou le milieu intérieur; mais le sang à son tour nourrit les organes qui le produisent. Il y a là une solidarité organique et sociale qui entretient dans l'économie animale un mouvement sans cesse dépensé et sans cesse renaissant, jusqu'à l'heure où le dérangement ou la cessation d'action d'un élément organique nécessaire amène un trouble dans le jeu de la machine vivante ou même en provoque l'arrêt définitif.

Le problème du médecin expérimentateur consiste donc à trouver le déterminisme simple d'un dérangement organique compliqué, c'està-dire à découvrir la condition du phénomène pathologique initial qui amène tous les autres à sa suite par un déterminisme complexe, qui n'est lui-même que l'enchaînement d'un plus ou moins grand nombre de déterminismos simples. 
Le déterminisme du phénomène initial une fois saisi sera le fil d'Ariane qui dirigera l'expérimentateur, et lui permeltra toujours de se retrouver dans le labyrinthe en apparence si obscur des phénomènes physiologiques et pathologiques. Il comprendra dès lors comment une succession de déterminismes subordonnés les uns aux autres engendre un ensemble logique de phénomènes se reproduisant toujours avec le même type comme des individualités appartenant à une espèce définie. A l'état physiologique, ces types de phénomènes constituent les fonctions; à l'état pathologique, ils forment les maladies. La production d'une maladie pour Van Helmont était due à l'évolution d'une idée morbide (idea febrilis), et pour les médecins d'aujourd'hui, c'est encore l'expression d'une entite morbide. Les empoisonnements comme les maladies se ramènent à un déterminisme complexe, ayant pour déterminisme initial l'action physico-chimique du poison sur un élément organisé, bien qu'il puisse ensuite, dans les déterminismes secondaires, intervenir des conditions de phénomènes qu'on peut appeler 
vilales, parce qu'elles ne se produisent pas en dehors de l'organisme vivant, sain ou malade ${ }^{2}$.

Enfin la connaissance du déterminisme physico-chimique initial des phénomènes complexes physiologiques ou pathologiques permettra seule au physiologiste d'agir rationnellement surles phénomènes de la vie et d'étendre sur eux sa puissance d'une manière aussi sùre que le font le physicien et le chimiste pour les phénomènes des corps bruts.

Toutefois il ne faudrait pas nous abuser sur notre puissance, car nous obéissons à la nature au lieu de lui commander. Nous ne pouvons en réalité connaître les phénomènes de la nature que par leur relation avec leur cause déterminante ou prochaine. Or la loi n'est rien autre chose que cette relation établie numéri-

1. Je pourrais citer beaucoup d'exemples pour prouver ce que j'avance. Je me bornerai à rappeler mes recherches sur l'action du curare dans lesquelles on peut voir comment la lésion physique d'une extrémité nerveuse motrice retenlit successivement sur tous les autres éléments vitaux, et amène des dẻterminismes secondaires qui vont se compliquant de plus en plus jusqu'à la mort. 
quement de manière à faire prévoir le rapport de la cause à l'effet dans tous les cas donnés. C'est ce rapport, établi par l'observation, qui permet à l'astronome de prédire les phénomènes célestes; c'est encore ce même rapport, établi par l'observation et par l'expérience, qui permet au physicien, au chimiste et au physiologiste non-seulement deprédire les phénomènes de la nature, mais encore de les modifier à son gré et à coup sûr, pourvu qu'il ne sorte pas des rapports que l'expérience lui a indiqués, c'està-dire de la loi. Ceci veut dire, en d'autres termes, que nous ne pouvons gouverner les phénomènes de la nature qu'en nous soumettant aux lois qui les régissent.

L'expérimentateur ne peut changer les lois de la nalure. Il agit sur les phénomènes, quand il en connaît le déterminisme physico-chimique; mais il ne lui est donné ni de les créer de toutes pièces ni de les anéantir absolument; il ne peut que les modifier. Les conditions physico-chimiques des phénomènes sont d'autant plus faciles à analyser et à préciser que le phénomène est plus simple; mais au fond et dans tous les cas; 
DANS LES SGIENCES PHYSIOLOGIOUES. 75 ainsi que nous l'avons dit, la cause première du phénomène reste entièrement impénétrable. L'expérimentateur peut donc plus qu'il ne sail, et, quelle que soit la manière dont son espr.t concoive les forces de la nature, vitales ou minérales, son problème est toujours le même: déterminer les conditions matérielles dans lesquelles un phénomène apparaît; puis, ces conditions étant connues, les réaliser ou non, pour faire apparaître ou disparaître le phénomène. Pour produire un phénomène nouveau, l'expérimentateur ne fait que réaliser des conditions phénoménales nouvelles; mais il ne crée rien, nicom me force ni comme matière.

A la fin du siècle dernier, la science a proclamé une grande vérité, à savoir qu'en fait de matière rien ne se perd ni rien ne se crée dans la nature; tous les corps, dont les propriétés varient sans cesse sous nos yeux, ne sont que des transmutations d'agrégats de matières équivalentes en poids.

Dans ces derniers temps, la science a proclamé une seconde vérité dont elle poursuit encore la démonstration, et qui est en quelque 
sorte le complément de la première, à savoir qu'en fail de forces rien ne se perd ni ne se créc dans la nature; d'où il suit que toutes les formes des phénomènes de l'univers, variées à l'infini, ne sont que des transformations équivalentes de forces les unes dans les autres.

Sans vouloir aborder ici la question de la nature des forces minérales et des forces vitales, qu'il me suffise de dire que les deux vérités que je viens d'énoncer sont universelles, et qu'elles embrassent les phénomènes des corps vivants aussi bien que ceux des corps bruts.

Comme conséquence de ce qui précède, nous voyons que tous les phénomènes, de quelque ordre qu'ils soient, existent virtuellement dans les lois immuables de la nature, et qu'ils ne se manifestent que lorsque leurs conditions d'existence sont réalisées.

Les corps et les êtres qui sont à la surface de notre terre expriment le rapport harmonicux des conditions cosmiques de notre planète et de notre atmosphère avec les êtres et les phénomènes dont elles permettent l'existence.

D’autres conditions cosmiques feraient néces- 
sairement apparaître un autre monde dans lequel se manifesteraient tous les phénomènes qui y rencontreraient leurs conditions d'existence, et dans lequel disparaîtraient tous ceux qui ne pourraient s'y développer; mais quelles que soient les variétés de phénomènes infinies que nous concevions sur la terre, en nous plaçant par la pensée dans toutes les conditions cosmiques que notre imagination peut enfanter, nous sommes toujours obligés d'admettre que tout cela se passera d'après les lois de la physique, de la chimie et de la physiologie, qui existent ì notre insu de toute éternité, et que dans tout ce qui arriverait il n'y aurait rien de créé ni en force ni en matière, qu'il y aurait seulement production de rapports différents, et par suite création d'êtres et de phénomènes nouveaux.

Quand un chimiste fait apparaître un corps nouveau dans la nature, il ne saurait se flatter d'avoir créé les lois qui l'ont fait naître; il n'a fait que réaliser les conditions qu'exigeait la loi créatrice pour se manifester. Il en est de même pour les corps organisés : un chimiste et un physiologiste ne pourraient faire apparaitre des 
êtres vivants nouveaux dans leurs expériences qu'en obéissant aux lois éternelles de la nature.

\section{III}

La méthode expérimentale a pour but de trouver le déterminisme ou la cause prochaine des phénomènes de la nature. Le principe sur lequel repose cette méthode est la certitude qu'un déterminisme existe; son procédé de recherche est le doute philosophique; son critérium est l'expérience. En d'autres termes, le savant croit d'une manière absolue à l'existence du déterminisme qu'il cherche, mais il doute toujours de l'avoir trouvé. C'est pour cela qu'il est sans cesse obligé de s'en référer à l'expérience. La méthode expérimentale n'est que l'expression de la marche naturelle de l'esprit humain allant à la recherche des vérités scientifiques qui sont hors de nous. Chaque homme se fait de prime 
DANS LES SGIENCES PHYSIOLOGIQUES. 79 abord des idées sur ce qu'il voit, et il est porté à interpréter les phénomènes de la nature par anticipation avant de les connaitre par expérience. Cette tendance est spontanée; une idée préconcue a toujours été et sera toujours le premier élan d'un esprit investigateur. La méthode expérimentale a pour objet de transformer cette conception à priori, fondée sur une intuition ou un sentiment vague des choses, en une interprétation à posteriori, établie sur l'étude expérimentale des phénomènes. C'est pourquoi on a aussi appelé la méthode expérimentale méthode à posteriori.

L'esprit humain a passé par trois périodes nécessaires dans son évolution. D'abord le scntiment, s'imposant à la raison, créa les vérités de la foi, c'est-à-dire la théologie. La raison ou la philosophie, devenant ensuite la maîtresse, enfanta les systèmes ou la scolastique. Enfin l'cxpérience, c'est-à-dire l'étude des phénomènes naturels, apprit à l'homme que les vérités du monde extérieur ne se trouvent formulées de prime abord ni dans le sentiment ni dans la raison. Ce sont seulement nos guides indispen- 
salıles; mais pour atteindre ces rérités, il faut nécessairement descendre dans la réalité objective des faits, où elles se trouvent sous la forme de relations phénoménales.

C'est ainsi qu'apparaît par le progrès naturel des choses la méthode expérimentale, qui résume tout en s'appuyant successivement sur les trois branches de ce trépied immuable: le sen. timent, la raison et l'expérience. Dans la recherche de la vérité au moyen de cette méthode, le sentiment a toujours l'initiative, il engendre l'idée à priori : c'est l'intuition. La raison ou le raisonnement développe ensuite l'idés et déduit ses conséquences logiques; mais si le sentiment doit être éclairé par les lumières de la raison, la raison à son tour doit être guidée par l'expérience, qui seule lui permet de conclure.

L'esprit humain est un tout complexe qui ne marche et ne fonctionne que par le jeu harmonique de ses diverses facultés.

Il faudrait donc se garder, dans l'association que j’ai signalée plus haut, de donner une prédominance exagérée soit au sentiment, soit à la raison, soit à l'expérience. Si le sentiment 
DANS LES SCIENGES PHYSIOLOGIQUES.

fait taire la raison, nous sommes hors de la science et nous arrivons dans les vérités irrationnelles de foi ou de tradition. Si la raison n'invoque pas sans cesse l'expérience, nous tombons dans la scolastique et sous la domination des systèmes; si l'expérience se passe du raisonnement, nous ne pouvons pas sortir des faits, et nous croupissons dans l'empirisme.

La méthode expérimentale est la méthode qui cherche la vérité par l'emploi bien équilibré du sentiment, de la raison et de l'expérience. Elle proclame la liberté de l'esprit et de la pensée. Son caractère est de ne relever que d'elle-même, parce qu'elle empruntè à son critérium, l'expérience, une autorité impersunnelle qui domine toute la science. Elle n'admet pas d'autorité personnelle; elle repousse d'une manière absolue les systèmes et les doctrines. Ceci n'est point de l'orgueil et de la jactance. L'expérimentateur au contraire fait acte d'humilité en niant l'autorité individuelle, car il doute de ses propres connaissances, et il soumet ainsi l'autorité des hommes à celle de l'expérience et des lois de la nature. 
La première condition à remplir pour un savant qui se livre à l'investigation expérimentale des phénomènes naturels, c'est donc de ne se préoccuper d'aucun système et de conserver une entière liberté d'esprit assise sur le doute philosophique. En effet, d'un côté nous avons la certitude de l'existence du déterminisme des phénomènes, parce que cette certitude nous est donnée par un rapport nécessaire de causalité dont notre esprit a conscience; mais nous n'avons, d'un autre côté, aucune certitude relativement à la formule de ce déterminisme, parce qu'elle se réalise dans des phénomènes qui sont en dehors de nous. L'expérience seule doit nous diriger; elle est notre critérium unique, et elle devient, suivant l'expression de Goethe ${ }^{1}$, la seule médiatrice qui existe entre le savant et les phénomènes qui l'environnent.

Une fois que la recherche du déterminisme des phénomènes est admise comme but unique de la méthode expérimentale, il n’y a plus ni

1. Goethe, Euvres d'histoire naturelle, traduction de M. Martins, introduction, p. 1. 
DANS LES SGIENGES PHYSIOLOGIQUES.

matérialisme, ni spiritualisme, ni matière brute, ni matière vivante, il n'y a que des phénomènes naturels dont il faut déterminer les conditions, c'est-à-dire connaître les circonstances qui jouent par rapport à ces phénomènes le rôle de cause prochaine. Toutes les sciences qui font usage de la méthode expérimentale doivent tendre à devenir antisystématiques.

La médecine expérimentale ne sera pas un système nouveau de médecine, mais au contraire la négation de tous les systèmes. Elle ne devra se rattacher à aucun mot systématique; elle ne sera ni animiste, ni organiciste, ni solidiste, ni humorale: elle sera simplement li science qui cherche à remonter aux causes prochaines des phénomènes à l'état sain et ì l'état morbide.

Ce que nous venons de dire relativement aus systèmes médicaux, nous pouvons l'appliquer aux systèmes philosophiques. La physiolog̣ie expérimentale ne sent le besoin de se rattacher à aucun système philosophique. Le rôle du physiologiste, comme celui de tout savant, est de chercher la vérité en elle-même, sans vou- 
loir la faire servir de contrôle à tel ou tel système de philosophie. Quand le savant poursuit l'investigation scientifique en prenant pour base un système philosophique quelconque, il s'égare nécessairement dans les régions des causes premières. L'idée systématique donne à l'esprit une sorte d'assurance trompeuse et une inflexibilité qui s'accordent mal avec la liberté du doute que doit toujours garder l'expérimentateur dans ses recherches. Les systèmes sont tous nécessairement incomplets; ils ne sauraient représenter tout ce qui est dans la nature, mais seulement ce qui est dans l'esprit des hommes. Or, pour trouver la vérité, il suffit que le.savint se mette en face de la nature, qu'il inter roge librement en suivant la méthode expérimentale à l'aide de moyens d'investigation de plus en plus parfaits, et je pense que dans ce cas le seul système philosophique consiste à ne pas en avoir.

Comme expérimentateur, j'évite donc les systimes philosophiques, mais je ne saurais pour cela repousser cet esprit philosophique qui, sans être nulle part, est partout, et qui, sans appar- 
tenir à aucun système, doit régner non-seulelement sur toutes les sciences, mais sur toutes les connaissances humaines. C'est ce qui fait que, tout en fuyant les systèmes philosophiques, j'aime beaucoup les philosophes, et je me plais infiniment dans leur commerce. En effet, au point de vue scientifique, la philosophie représente l'aspiration éternelle de la raison humaine vers la connaissance de l'inconnu. Dès lors les philosophes se tiennent toujours dans les questions en controverse et dans les régions élevées, limites supérieures des sciences. Par là ils communiquent à la pensée scientifique un mouvement qui la vivifie et l'ennoblit; ils fortifient l'esprit en le dévelopfant par une gymnastique intellectuelle générale en même temps qu'ils le reportent sans cesse vers les solutions inépuisables des grands problèmes; ils entretiennent ainsi une sorte de soif de l'inconnu et le feu sacré de la recherche qui ne doivent jamais s'éteindre chez un savant.

En effet, le désir ardent de la connaissance est l'unique mobile qui attire et soutient l'investigateur dans ses efforts, et c'est précisément 
celte connaissance qu'il saisit et qui fuit toujour's devant lui, qui devient à la fois son seul tourment et son seul bonheur. Celui qui ne connaît pas les tourments de l'inconnu doit ignorer les jöies de la découverte, qui sont certainement les plus vives que l'esprit de l'homme puisse jamais ressentir.

Mais, par un caprice de notre nature, cette joie de la découverte tant cherchée et tant espérée s'évanouit dès qu'elle est trouvée. Ce n'est qu'un éclair dont la lueur nous a découvert d'autres horizons vers lesquels notre curiosité inassouvie se porte encore avec plus d'ardeur. C'est ce qui fait que, dans la science même, le connu perd son attrait, tandis que l'inconnu est toujours plein de charmes. C'est pour cela que les esprits qui s'élèvent et deviennent vraiment grands sont ceux qui ne sont jamais satisfaits d'eux-mêmes dans leurs œuvres accomplies, mais qui tendent toujours à mieux dans des ouvres nouvelles.

Le sentiment dont je parle en ce moment est bien connu des savants et des philosophes. 
DANS LES SCIENGES PHYSIOLOGIQUES.

C'est ce sentiment qui a fait dire à' Priestley ${ }^{1}$ qu'une découverte que nous faisons nous en montre beaucoup d'autres à faire; c'est ee sentiment qu'exprime Pascal ${ }^{2}$, mais sous une forme peut-être paradoxale, quand il dit : “ Nous ne cherchons jamais les choses, mais la recherche des choses. "

Pourtant c'est bien la vérité elle-même qui nous intéresse, et si nous la cherchons toujours, c'est parce que ce que nous en avons trouvé ne peut pas nous satisfaire. Sans cela, nous ferions dans nos recherches ce travail inutile et sans fin que nous représente la fable Sisyphe, qui roule toujours son rocher qui retombe sans cesse au point de départ. Cette comparaison n'est point exacte scientifiquement: le savant monte toujours en cherchant la vérité, et s'il ne la trouve jamais tout entière, il en découvre néanmoins des fragments très-importants, et ce sont précisément ces lambeaux de la vérité générale qui constituent la science.

1. Priestley, Expériences et observations sur différentes espèces d'ain's, t. Ier, préface, p. 15.

2. Pascal, Pensées morales détachées, art. Ix-xxwiv. 
Le savant ne cherche done pas pour le plaisir de chercher, mais pour le plaisir de trouver. Il cherche la vérité à cause du désir ardent qu'il a de la posséder, et il la possède déjà dans des limites qu'expriment les sciences ellesmêmes dans leur état actuel. Mais le savant ne doit pas s'arrêter en chemin : il doit toujours s'élever plus haut et tendre à la perfection, il doit toujours chercher tant qu'il voit quelque chose à trouver. Sans cette excitation constante qui est donnée par l'aiguillon de l'inconnu, sans cette soif scientifique toujours renaissante, il serait à craindre que le savant ne se systématisât dans ce qu'il a d'acquis ou de connu. Alors la science ne ferait plus de progrès et s'arrêterait par ¿indifférence intellectuelle, comme quand les corps minéraux saturés tombent en indifférence chimique et se cristallisent.

I] faut donc empêcher que l'esprit, trop absorbé par le connu d'une science spéciale, ne tende au repos ou ne se traîne terre à terre, en perdant de vue les questions qui lui restent à résoudre. La philosophie, en agitant la masse inćpnisable des questions non résolıes, stimule 
DANS LES SCIENCES PHYSIOLOGIQUES.

et entretient ce mouvement salutaire dans les sciences, car, dans le sens restreint où je considère ici la philosophie, l'indéterminé seul lui appartient, le déterminé retombant nécessairement dans le domaine scientifique. Je n'admets donc pas la philosophie qui voudrait assigner des bornes à la science, pas plus que la science qui prétendrait supprimer les vérités philosophiques qui sont actuellement hors de son propre domaine. La vraie science ne supprime rien, elle cherche toujours et regarde en face et sans se troubler les choses qu'elle ne comprend pas encore. Nier ces choses ne serait pas les supprimer; ce serait fermer les yeux et croire que la lumière n'existe pas. Ce serait l'illusion de l'autruche qui croit supprimer le danger en se cachant la tête dans le sable. .

Selon moi, le véritable esprit philosophique est celui dont les aspirations élevées fécondent les sciences en les entraînant à la recherche de vérités qui sont actuellement en dehors d'elles, mais qui ne doivent pas être délaissées par cela nıême qu'elles s'éloignent et s'élèvent de plus en plus à mesure qu'elles sont abordées par des 
esprits phiiosophiques plus puissants et plus délicats. Maintenant cette aspiration de l'esprit humain aura-t-elle une fin, trouvera-t-elle une limite? Je ne saurais le comprendre; en attendant, le savant n'a rien de mieux à faire que de marcher sans cesse, parce qu'il avance toujours.

Un des plus grands obstacles qui se rencontrent dans cette marche générale et libre des connaissances humaines est donc la tendance qui porte les diverses connaissances à s individualiser dans des systèmes. Cela n'est point une conséquence des choses elles-mêmes, parce que dans la nature tout se tient et que rien ne saurait être vu isolément et systématiquement, mais c'est un résultat de la tendance de notre esprit, à la fois faible et dominateur, qui nous porte à absorber les autres connaissances dans une systématisation personnelle. Une science qui s'arrêterait dans un système resterait stationnaire et s'isolerait, car la systématisation est un véritable enkystement scientifique, et toute partie enkystée dans un organisme cesse de participer à la vie générale de cet organisme. 
Les systèmes tendent doric à asservir l'esprit humain, et la seule utilité que l'on puisse, suivant moi, leur trouver, c'est de susciter des combats qui les détruisent en agitant et en excitant la vitalité de la science. En effet, il faut chercher à briser les entraves des systèmes philosophiques et scientifiques, comme on briserait les chaînes d'un esclavage intellectuel. La vérité, si on peut la trouver, est de tous les systèmes, et pour la découvrir l'expérimentateur' a besoin de se mouvoir librement de tous les côtés sans se sentir arrété par les barrières d'un système quelconque. La philosophie et la science ne doivent donc pas être systématiques, elles doivent être unies et s'entr'aider sans vouloir se dominer l'une l'autre.

Mais si, au lieu de se contenter de cette union fraternelle pour la recherche de la vérité, la philosophie voulait entrer dans le ménage de la science et lui imposer dogmatiquement des méthodes et des procédés d'investigation, l'accord ne pourrait certainement plus exister. Pour faire des observations, des expériences ou des découvertes scientifiques, les méthodes et pro- 
cédés philosophiques sont trop généraux et restent impuissants; il n'y a pour cela que des méthodes et des procédés scientifiques souvent très-spéciaux qui ne peuvent être connus que des expérimentateurs, des savants ou des philosophes qui pratiquent une science déterminée.

Les connaissances humaines sont tellement enchevêtrées et solidaires les unes des autres dans leur évolution, qu'il est impossible de croire qu'une influence individuelle puisse suffire à les faire avancer lorsque les éléments du progrès ne sont pas dans le sol scientifique luimême. C'est pourquoi, tout en reconnaissant la supériorité des grands hommes, je pense néanmoins que, dans l'influence particulière ou générale qu'ils ont sur les sciences, ils sont toujours et nécessairement plus ou moins fonclion de leur temps.

Il en est de même des philosophes : ils ne peuvent que suivre la marche de l'esprit humain, et ils ne contribuent à son avancement qu'en attirant les esprits vers la voie du progrès, que beaucoup n'apercevraient peut-être pas; mais ils sont encore en cela l'expression de leur 
DANS LES SGIENGES PHYSIOLOGIOUES.

temps. Ce serait done une illusion que de prétendre absorber les découvertes particulières d'une science au profit d'une méthode ou d'un système philosophique quelconque. En un mol, si les savants sont utiles aux philosophes et les philosophes aux savants, le savant n'en reste pas moins libre et complétement maître chez lui, et je pense, quant à moi, que les savants dans leurs laboratoires font leurs découvertes, leurs théories et leur science sans les philosophes. Joseph de Maistre a dit que ceux qui ont fait le plus de découvertes dans la science sont ceux qui ont le moins connu Bacon ${ }^{1}$; ceux qui l'ont lu et médité, ainsi que Bacon lui-même, n'y ont souvent guère réussi.

C'est qu'en effet l'art d'obtenir le déterminisme des phénomènes à l'aide des procédés et des méthodes scientifiques ne s'apprend que dans les laboratoires, où l'expérimentateur est aux prises avec les problèmes de la nature. Quand on est en face de phénomènes dont il

1. Joseph de Maistre, Examen de la Philosophic de Bucon, t. I ${ }^{\mathrm{or}}$, p. 81. 
faut déterminer les conditions d'existence ou les causes prochaines, les procédés du raisonnement doivent varier à l'infini, suivant la nature des phénomènes dans les diverses sciences et selon les cas plus ou moins difficiles et plus ou moins complexes auxquels on les applique. Les savants, et même les savants spéciaux en chaque science, peuvent seuls intervenir dans de pareilles questions, parce que non-seulement les procédés diffèrent, mais parce que l'esprit du naturaliste n'est pas celui du physiologiste, et que celui du chimiste n'est pas celui du physicien.

Quand des philosophes tels que Bacon, ou d'autres plus modernes, ont voulu donner une systématisation de préceptes pour la recherche scientifique, ils ont pu paraître séduisants aux personnes qui ne voient les sciences que de loin; mais en réalité de pareils ouvrages ne sont d'aucune utilité aux savants faits, et pour ceux qui veulent se livrer à la culture des sciences, ils les égarent par une fausse simplicité des choses; bien plus, ils les gênent en chargeant l'esprit d'une foule de règles vagues 
DANS LES SGIENGES PHYSIOLOGIQUES.

ou inapplicables, qu'il faut se hâter d'oublier, si l'on veut entrer dans la science et devenir un véritable expérimentateur.

Je crois que dans l'enseignement scientifique le rôle d'un maître est de montrer expérimentalement à l'élève le but que le savant se propose, et de lui indiquer tous les moyens qu'il peut avoir à sa disposition pour l'atteindre. Le maître doit ensuite laisser l'élève libre de se mouvoir à sa manière, suivant sa nature, pour arriver au but qu'il lui a montré, sauf ì venir à son secours, s'il voit qu'il s'égare. Je pense enfin que la vraie méthode scientifique est celle qui contient l'esprit sans l'étouffer, celle qui laisse autant que possible l'esprit en face de lui-même, et le dirige tout en respectant ses qualités les plus précieuses qui sont son originalité créatrice et sa spontanéité scientifique. En effet, les sciences n'avancent que par les idées nouvelles et par la puissance créatrice ou originale de la pensée. Il faut donc prendre garde, dans l'enseignement des sciences, que les connaissances qui doivent armer l'intelligence ne l'accablent par leur poids, et 
que les règles qui sont destinćes à soutenir les côtés faibles de l'esprit n'en atrophient ou n'en étouffent les côtés puissants et féconds.

Je n'ai point à entrer ici dans d'autres dére. loppements; j’ai dû me borner à prémunir les sciences physiologiques et la médecine expérimentale contre les exagérations de l'érudition et contre l'envahissement et la domination des systèmes, parce que ces sciences, en y succombant, verraient disparaître leur fécondité, et perdraient l'indépendance et la liberté d'es. prit, qui seront toujours les conditions essen. tielles de leurs progrès.

Si le génie de l'homme a dans les sciences comme ailleurs une suprématie qui ne perd jamais ses droits, cependant, pour les sciences expérimentales, le savant doit appliquer ses idées à la rešlerche du déterminisme scientifique et interroger la nature dans un laboratoire, avec les moyens convenables et nécessaires. On ne concevrait pas un physicien ou un chimiste sans laboratoire. Pour le physiologiste il doi: en être de même : il faut qu'il analyse expérimentalement les phénomènes de la matière 
DANS LES SGIENGES PIIYSIOLOGIQUES.

vivante, comme le physicien et le chimiste analysent expérimentalement les phénomènes de la matière brute. En un mot, le laboratoire est la condition sine qua non du développement de toutes les sciences expérimentales.

L'évidence de celte vérité amène et amènera nécessairement une réforme universelle et profonde dans l'enseignement scientifique, car on a reconnu partout aujourd'hui que c'est dans les laboratoires que germent et grandissent toutes les découvertes de la science pure, pour se répandre ensuite et couvrir le monde de leurs applications utiles. Le laboratoire seul apprend les difficultés réelles de la science à ceux qui le fréquentent. Il leur montre en outre que la science pure a toujours été la source de toutes les richesses réelles que l'homme acquiert el de toutes les conquêtes qu'il fait sur les phéno mènes de la nature. C'est là une excellente éducation pour la jeunesse, parce qu'elle scule peut lui faire comprendre que les applicalions actuelles si brillantes des sciences ne sont que l'épanouissement de travaux antérieurs, et que ceux qui profitent aujourd'hui de leurs bienfaits CLAUDE BERNARD. 
98 PROGRĖS DANS LES SGIENCES PHYSIOLOGIQUES. doivent un tribut de reconnaissance à leurs devanciers, qui ont péniblement cultivé l'arbre de la science sans le voir fructifier.

$1^{\text {er }}$ août 1865. 


\section{LE PROBLÈME}

\section{DE LA PHYSIOLOGIE GÉNÉRALE}

On distingue les sciences qui traitent des corps inertes de celles qui traitent des corps vivants, et, parmi ces dernières, on sépare encore celles qui étudient l'homme et les animaux de celles qui étudient les végétaux.

Toutes les classifications des sciences ne sauraient se fonder exclusivement sur les circonscriptions naturelles des corps qu'elles considèrent; elles se divisent aussi et plus particulièrement selon les problèmes spéciaux qu'elles se proposent de résoudre. La physiologie générale, par son objet, se confond avec toutes les sciences des êtres vivants, puisqu'elle analyse 
des phénomènes qui se passent à la fois dans l'homme, dans les animaux et dans les végétaux ${ }^{1}$. Elle n'en est pas moins cependant une science distincte, parce qu'elle poursuit un problème spécial qui détermine son domaine propre.

La physiologie a pour but de régir les manifestations des phénomènes de la vie. Je me propose ici d'examiner comment il est possible d'arriver à la solution d'un pareil problème. On verra, je l'espère, que la physiologie est une des sciences les plus dignes de l'attention des esprits élevés par l’importance des questions qu'elle traite, et de toute la sympathie des hommes de progrès par l'influence qu'elle est destinée à exercer sur le bien-être de l'humanité.

1. Gl. Bernard, Lecons sur les phénomènes de la vie communs aux animaux ct aux végétaux. Paris, 1878. 


\section{I}

Afin de bien comprendre le caractère du problème physiologique, il faut d'abord circonscrire la physiologie générale et montrer qu'elle est une science expérimentale et non une science naturelle.

Les sciences naturelles sont des sciences d'observation ou descriptives. Elles nous donnent la prévision des phénomènes; mais elles restent des sciences contemplatives de la nature.

Les sciences expérimentales sont des sciences d'expérimentation ou explicatives. Elles vont plus loin que les sciences d'observation, qui leur servent de base, et arrivent à être des sciences d'action, c'est-à-dire des sciences conquérantes de la nature.

Cette distinction fondamentale entre les 
sciences naturelles et les sciences expérimentales ressort de la définition même de l'observation et de l'expérimentation. L'observateur considère les phénomènes dans leur état naturel, c'est-à-dire tels que la nature les lui offre, tandis que l'expérimentateur les fait apparaître dans des conditiorıs dont il est le maître.

La physique et la chimie, qui sont les sciences expérimentales dans le règne des corps bruts, cnt conquis la nature inerte ou minérale, et chaque jour nous voyons cette conquête s'étendre davantage.

La physiologie, qui est la science expérimentale dans le règne des corps organisés, doit conquérir la nature vivante; c'est là son problème, ce sera là sa puissance.

Cette division des sciences biologiques en sciences naturelles et en sciences expérimentales est nécessaire à leurs progrès.

D'un côté, la physiologie ne peut avancer qu'en se constituant comme une science indépendante, et d'autre part les sciences naturelles qui ont concouru à son évolution et prẻparé son avénement feraient fausse route, et per- 
DE LA PHYSiologie GÉNÉRALE.

draient leur véritable point de vue, soit en voulant la suivre dans sa marche, soit en essayant de la retenir dans leur circonscription. Par la même raison, les naturalistes, minéralogistes et géologues pourraient réclamer la physique et la chimie comme appartenant à l'histoire des minéraux. De même encore le naturaliste anthropologiste devrait, ainsi que cela d'ailleurs a été fait par certains auteurs, considérer la physiologie humaine et la médecine comme ne formant que des divisions de l'anthropologie. On sent tout de suite combien il serait facile de pousser jusqu'à l'erreur de semblables raisonnements, car la littérature, les arts, la politique, toutes les connaissances humaines, en un mot, appartiendraient à l'anthropologie, puisqu'elles rentrent dans l'histoire de l'intelligence de l'homme. Cette manière de diviser les sciences d'après la considération de. l'objet qu'on étudie n'aboutirait qu'à l'obscurité et à la confusion, tandis qu'en envisageant la nature expérimentale et spéciale des problèmes du physiologiste, nous verrons qu'on peut arriver au contraire à une distinction réelle et féconde. 
Cuvier a donné à la science de l'organisation des êtres vivants une impulsion puissante, qui a été utile à la fois à la zoologie et à la physiologie générale; mais Curier ne concevait pas la physiologie comme devant être une science expérimentalement constituée, ou plutôt il n'avait pas d'idée arrêtée à ce sujet, car tantôt on le voit nier la physiologie expérimentale en contestant la légitimité des applications de la méthode expérimentale à l'étude des phénomènes de la vie ${ }^{1}$, tantôt on le voit admettre et louer dans des rapports académiques les résultats de la physiologie expérimentale obtenus à l'aide de la vivisection ${ }^{2}$. Cuvier avait bien senti qu'il était important d'introduire les considérations physiologiques dans la zoologie; mais il n'était pas physiologiste, il était naturaliste et surtout anatomiste, et ne voyait dans la phy-

1. Voyez Cuvier, Lettre à Mertrud et Introduction au rigne animal.

2. Voyez Cuvier, Rapport fait à l'Académie des Sciences sur des expériences relatives aux fonctions du syslème nereeux (Joumal de Physiologie, par Nagendie, I. II, p. 372, 1822). 
siologie que des déductions anatomiques particulières dont il cherchait la confirmation dans l'anatomie comparée. Sans doute les connaissances anatomiques les plus précises sont indispensables au physiologiste; mais je ne crois pas pour cela avec les anatomistes que l'anatomie doive servir de base exclusive à la physiologie, et que cette dernière science puisse jamais se déduire directement de la première' . Je pense au contraire que c'est une erreur ou une illusion de toutes les écoles anatomiques d'avoir cru que l'anatomie expliquait directement la physiologie.

L'impuissance de l'anatomie à nous apprendre les fonctions organiques devient surtout évidente dans les cas particuliers où elle est réduite à elle-même. Pour les organes sur les usages desquels la physiologie expérimentale n'a encore rien dit, l'anatomie reste absolument muette.

C'est ce qui a lieu par exemple pour la rate,

1. Voyez mes Lecons de physiologie appliquée à la midecine faites au Collége de France, 1855, première leçon. 
les capsules surrénales, le corps thỵroïde, etc., tous organes dont nous connaissons parfaitement la texture anatomique, mais dont nous ignorons complétement les fonctions.

De même, quand sur un animal on découvre un tissu nouveau et sans analogue dans d'autres organismes, l'anatomie est incapable d'en dévoiler les propriétés vitales.

Cela prouve donc bien clairement que, lorsque l'anatomiste ou le zoologiste construit ce qu'on appelle la physiologie anatomique ou zoologique, ils ne font qu'appliquer à l'interprétation et au classement des faits anatomiques les connaissances que leur a préalablement fournies la physiologie expérimentale, mais ils ne déduisent jamais rien directement de l'anatomie elle-même.

Pour expliquer les phénomènes de la vie, le physiologiste expérimentateur s'adresse directement aux manifestations de ces phénomènes; il les analyse à l'aide des sciences physico-chimiques, qui sont plus simples que la physioiogie, parce c'est toujours le plus simple qui doit éclairer le plus complexe. L'anatomie ou la 
texture d'un organe ne peut réellement se comprendre que lorsque la physiologie vient l'expliquer. La structure anatomique ne donnant que les conditions de manifestations d'un phénomène physiologique, il est de toute nécessité de connaître ce phénomène avant de chercher à l'expliquer anatomiquement. En un mot, la physiologie n'est point une déduction de l'anatomie. L'explication de l'organisation, au lieu d'être le point de départ, est au contraire le but vers lequel tendent toutes les études physiologiques. Nous verrons en effet que c'est seulement dans la structure anatomique et dans l'analyse physico-chimique des propriétés de la matière organisée que le physiologiste trouve les conditions qu'il lui importe de connaître pour résoudre le problème de la physiologie expérimentale, c'est-à-dire pour expliquer' le mécanisme des phénomènes vitaux et pour en maîtriser les manifestations.

Le problème du naturaliste est plus simple; sans chercher à expliquer les phénomènes naturels, il se borne à en constater l'enchaînement 
ct les lois, afin d'en prévenir les manifestations et la marche.

Les sciences naturelles et les sciences expérimentales, considérées dans leur développement, constituent en quelque sorte deux degrés dislincts dans les connaissances humaines. Les sciences naturelles, passives ou contemplatives, forment évidemment le premier degré, tandis que les sciences expérimentales, actives et conquérantes, constituent le second. Les sciences naturelles sont les aînées nécessaires des sciences expérimentales et elles leur servent de point d'appui.

C'est ainsi que l'évolution scientifique vient nous expliquer comment le problème des sciences expérimentales est un problème moderne que l'antiquité n'a pu connaître. Je ne veux pas dire que l'antiquité n'ait point eu l’idée de conquérir la nature, puisqu'elle nous a laissé la fable de Prométhée, puni pour avoir voulu ravir le feu du ciel. Seulement il est cer * lain que la science antique n'a pu réaliser cette conquête, puisque les sciences naturelles et contemplatives ont dû se former les premières. 
La pensée scientifique des anciens n'a done pu être que de découvrir et de constater les lois qui régissent les phénomènes de la nature, tandis que la pensée scientifique expérimentale moderne doit être d'expliquer ces phénomènes et de les maîtriser au profit de l'humanité. Nous savons que par la physique et par la chimie l'homme a déjà assuré sa domination sur les phénomènes des corps bruts; mais une autre conséquence également nécessaire de l'évolution scientifique que j'ai voulu proclamer ici, c'est que par la physiologie l'homme doit ambitionner aussi d'étendre sa puissance sur les phénomènes des êtres vivants.

La civilisation moderne, en conquérant par la science la nature inorganique et la nature organisée, se trouvera placée dans des conditions nouvelles entièrement inconnues aux civilisations antiques. C'est pourquoi il n'est peut-être pas toujours logique d'invoquer l'histoire des peuples anciens pour supputer les destinées des peuples nouveaux. L'humanité semble avoir compris aujourd'hui que son but est non plus la contemplation passive, mais le progrès et Claude BERNARD. 
l'action. Ces idées pénètrent de plus en plus profondément dans les sociétés, et le rôle actif des sciences expérimentales ne s'arrête pas aux sciences physico-chimiques et physiologiques; il s'étend jusqu'aux sciences historiques et morales. On a compris qu'il ne suffit pas de rester spectateur inerte du bien et du mal, en jouissant de l'un et se préservant de l'autre. La ‘morale moderne aspire à un rôle plus grand : elle recherche les causes, veut les expliquer et agir sur elles; elle veut en un mot dominer le bien et le mal, faire naître l'un et le développer, lutter avec l'autre pour l'extirper et le détruire. On le voit donc, c’est une tendance générale, et le souffle scientifique moderne qui anime la physiologie est éminemment conquérant et dominateur. 


\section{II}

De tout temps, les phénomènes de la vie ont été considérés sous deu faces différentes et pour ainsi dire opposées.

Les physiologistes animistes ou vitalistes ont pensé que les manifestations vitales sont régies par des influences spéciales, et ils ont admis que la force vitale, quel que soit le nom qu'on Jui donne (ime physiologique ou archée, principe vital ou propriétés vitales), est essentiellement distincte des forces minérales, et se tient même avec elles dans un antagonisme constant.

Les physiologistes chimistes physico-mécaniciens ont soutenu au contraire que les fonctions vitales doivent se ramener à des phénomènes mécaniques ou physico-chimiques ordinaires, pour l'explication desquels il n'est nécessaire de faire intervenir aucune force vitale particulière. 
En voyant que nous considérons la physiologie comme une science expérimentale destinée à gouverner les phénomènes de la nature vivante, on se demandera si nous sommes dans le camp des physiologistes vitalistes ou dans celui des physiologistes physico-mécaniciens. Il devient par conséquent nécessaire de nous expliquer, non afin de prendre parti pour l'une ou l'autre des deux doctrines physiologiques précédemment citées, mais simplement afin de faire connaître notre manière de voir sur la na. ture des phénomènes de la vie et sur la méthode d'investigation qu'il convient de suivre dans l'étude des problèmes de la physiologie générale.

La physiologie ne se sépare pas, quant à la manière d'étudier, des autres sciences expérimentales des corps bruts. Elle suit la même méthode expérimentale, et la vie, quelle que soit l'idée qu'on s'en fasse, ne saurait être un obstacle à l'analyse expérimentale des phénomènes des organismes vivants. J'ai déjà développé ${ }^{1}$ cette opinion, et j'ai démontré par di-

1. Voyez : Du progrès dans les sciences physiologiques, p. 37. 
vers exemples que les phénomènes vitaux sont soumis à un déterminisme aussi rigoureux et aussi absolu que les phénomènes minéraux. Quant aux phénomènes de la vie, j'admets que ces phénomènes, considérés dans leurs formes diverses de manifestation et dans leur nature intime, ont à la fois une spécialité de formes qui les distingue comme phénomènes de la vie et une communauté de lois qui les confond avec tous les autres phénomènes du monde cosmique. Je reconnais en d'autres termes à tous les phénomènes vitaux des procédés spéciaux de manifestation; mais en même temps je les considère aussi comme dérivant tous des lois générales de la mécanique et de la physico-chimie ordinaires.

Il existe en effet dans les organismes vivants des appareils anatomiques ou des outils organiques qui leur sont propres, et qu'on ne saurait reproduire en dehors d'eux; mais les phénomènes manifestés par ces organes ou tissus vivants n'ont cependant rien de spécial ni dans leur nature, ni dans les lois qui les régissent : c'est une proposition que les progrès des scien- 
res physico-chimiques démontrent chaque jour de plus en plus, en prouvant que les phénomènes qui s'accomplissent dans les corps vivants peuvent s'accomplir également en dehors de l'organisme dans le règne minéral. Dans l'orIre chimique, le chimiste opère dans son laboratoire une foule de synthèses, de décompositions et de dédoublements semblables à ceux qui ont lieu dans les organismes animaux et végétaux; mais, si dans l'être vivant les forces chimiques donnent lieu à des produits identiques à ceux du règne minéral, la nature vivante emploie les procédés spéciaux des éléments histologiques (cellules ou fibres organisées) qui n'appartiennent qu'aux êtres vivants. Parmi les cellules organiques animales ou végétales, il en est qui réduisent l'acide carbonique et dégagent de l'oxygène, d'autres qui absorbent l'oxygène et dégagent de l'acide carbonique; enfin certaines cellules ou produits de cellules (ferments solubles) président à des phénomènes de fermentation ou de dédouble. ment qui donnent naissance à de l'alcool, à de l'acide acétique, à des acides gras, à de la gly- 
cérine, à de l'urée, à des essences végétales, etc. Or ce sont là des phénomènes et des produits que le chimiste peut imiter et refaire dans son laboratoire en mettant en jeu les forces chimiques minérales, qui sont au fond exactement les mêmes que les forces chimiques organiques; mais dans l'être vivant, je le répète, les phénomènes sont réalisés à l'aide de procédés vitaux et de réactifs chimiques organisés, créés par l'évolution histologique et par conséquent spéciaux à l'organisme et inimitables pour le chimiste.

Dans l'ordre mécanique ou physique, les phénomènes de l'organisme vivant n'ont rien non plus qui les distingue des phénomènes mécaniques ou physiques généraux, si ce n'est les instruments qui les manifestent.

Le muscle produit des phénomènes de mouvement qui, comme ceux des machines inertes, ne sauraient échapper aux lois de la mécanique générale, ce qui n’empêche pas que le muscle ne soit un appareil de mouvement spécial ì l'animal, et dont le jeu est régglé par les nerfs 
au moyen de mécanismes éģalement spéciaux à l'être vivant.

Les êtres vivants produisent de la chaleur qui ne diffère en rien de la chaleur engendrée dans les phénomènes minéraux, si ce n’est le procédé vital de fermentation ou de combustion qui lui donne naissance.

Les poissons électriques forment ou sécrètent de l'électricité qui ne diffère en rien de l'électricité d'une pile métallique, ce qui n'empêche pas l'organe électrique de la torpille, par exemple, d'être un appareil vital tout à fait particulier, réglé par le système nerveux et que le physicien ne peut imiter.

Il en serait de même des fonctions des nerfs et des organes des sens, qui ne sont que des instruments de physique spéciaux aux êtres vivants.

Il n'y a donc en réalité qu'une physique, qu'une chimie et qu'une mécanique générales, dans lesquelles rentrent toutes les manifestations phénoménales de la nature, aussi bien celles des corps vivants que celles des corps bruts. Tous les phénomènes, en un mot, qui 
apparaissent dans un être vivant retrouvent leurs lois en dehors de lui, de sorte qu'on pourrait dire que toutes les manifestations de la vie se composent de phénomènes empruntés, quant à leur nature, au monde cosmique extérieur, mais possédant seulement une morphologie spéciale, en ce sens qu'ils sont manifestés sous des formes caractéristiques et à l'aide d'instruments physiologiques spéciaux. Sous le rapport physico-chimique, la vie n'est donc qu'une modalité des phénomènes généraux de la nature; elle n'engendre rien; elle emprunte ses forces au monde extérieur, et ne fait qu'en varier les manifestations de mille et mille manières. Ne pourrait-on pas ajouter que l'intelligence elle-même, dont les phénomènes caractérisent l'expression la plus élevée de la vie, se révèle en dehors des êtres vivants dans l'harmonie des lois de l'univers? Mais nulle part ailleurs que dans les corps vivants elle n'est traduite par des instruments qui nous la manifestent sous la forme de sensibilité, de volonté. Ainsi se trouverait réalisée la pensée antique, que l'organisme vivant est un microcosme (petit 
monde) qui reflète en lui le macrocosme (grand monde, l'univers).

De ce qui précède, il résulte évidemment que le physiologiste, le chimiste, le physicien, n'ont en réalité à considérer que des phénomènes de même nature, qui doivent être analysés et étudiés par la même méthode et réduits aux mêmes lois générales. Seulement le physiologiste a affaire à des procédés particuliers qui sont inhérents à la matière organisée, et qui constituent par conséquent l'objet spécial de ses études. La physiologie générale se trouve ainsi ramenée à être la science expérimentale qui étudie les propriétés de la matière organisée et explique les procédés et les mécanismes des phénomènes vitaux, comme la physique et la chimie expliquent les procédés et les mécanismes des phénomènes minéraux.

Si maintenant le physiologiste expérimentateur veut arriver à régir 'les phénomènes physiologiques dans l'être vivant, comme le physicien et le chimiste gouvernent les phénomènes physico-chimiques dans la nature inorganique, 
son problème sera réduit exactement aux mêmes termes.

En effet, le physicien et le chimiste rattachent l'explication des phénomènes aux propriétés des éléments inorganiques.

De même le physiologiste doit rechercher dans l'être vivant les éléments organiques dans lesquels se localisent les fonctions, et déterminer les conditions d'activité vitale de ces éléments sur lesquels il peut agir. Les éléments organiques des corps vivants sont les éléments anatomiques ou histologiques dans lesquels se décomposent nos organes et nos tissus. La science de l'organisation en est arrivée aujourd'hui à montrer qu'un corps vivant, quelle qu'en soit la complexité, est toujours constitué par la réunion d'un nombre plus ou moins considérable d'organismes élémentaires microscopiques dont les propriétés vitales diverses manifestent les différentes fonctions de l'organisme total ${ }^{4}$. Il résulte de là que chaque fonction doit avoir son élément organique correspondant, et l'objet de

1. Voyez: Le Curare, p. 237. 
la physiologie générale est précisément d’analyser les mécanismes fonctionnels complexes pour les ramener à leurs éléments vitaux particuliers. C'est ainsi que les phénomènes de sensibilité et de mouvement s'expliquent par les propriétés des éléments nerveux et musculaires, que les phénomènes de respiration et de sécrétion se déduisent des propriétés des éléments respiratoires du sang et des propriétés des éléments glandulaires et épithéliaux.

Les éléments organiques des êtres vivants, qui se présentent généralement sous les formes diverses de fibres ou de cellules microscopiques, sont les véritables ressorts cachés de la machine vivante. Ils sont associés et reliés entre eux pour former les tissus, les organes et les appareils qui constituent les rouages des mécanismes vitaux. Il y a de plus dans tout organisme vivant un véritable milieu intérieur dans lequel les éléments anatomiques remplissent leurs fonctions spéciales et parcourent toutes les phases de leur existence.

La matière organisée ou vivante, qui constitue les éléments histologiques, n’a pas plus de 
spontanéité que la matière inorganique ou minérale, car l'une et l'autre ont besoin, pour manifester leurs propriétés, de l'influence des excitants extérieurs. La spontanéité des corps vivants n'est qu'apparente ${ }^{1}$, et ne saurait s'opposer en rien à l'application de la méthode expérimentale et à l'analyse des phénomènes vitaux. L'expérimentateur physiologiste peut donc agir sur les propriétés de la matière organisée, et par conséquent sur les manifestations de la vie; mais nous allons voir de plus que ce sont absolument les mêmes agents ou les mêmes influences qui excitent les propriétés de la matière organique et celles de la matière inerte.

Les excitants généraux, air, chaleur, lumière, électricité, qui provoquent les manifestations des phénomènes physico-chimiques de la matière brute, éveillent aussi d'une manière parallèle l'activité des phénomènes propres à la matière vivante.

Lavoisier avait dejjà montré clairement que

1. Voyez : Du Progrès des Sciences physiologiques, p. 37. 
les phénomènes physico-chimiques des êtres vivants sont entretenus par les mêmes causes que ceux des corps minéraux. Il démontra que les animaux qui respirent et les métaux que l'on calcine absorbent dans l'air le même principe actif ou vital, l'oxygène, et que l'absence de cet air respirable arrête la calcination aussi bien que la respiration. Dans un autre travail, Lavoisier et Laplace prouvèrent que l'oxygène, en pénétrant dans les êtres vivants, engendre en eux la chaleur organique qui les anime par une véritable combustion semblable à la combustion de nos foyers. L'antique fiction de la vie comparée à une flamme qui brille et s'éteint cessa d'être une simple métaphore pour devenir une réalité scientifique. Ce sont en effet les mêmes conditions chimiques qui alimentent le feu dans la nature inorganique et la vie dans la nature organique.

Si, partant du fait signalé par Lavoisier, nous descendons maintenant dans l'analyse expérimentale des fonctions vitales, nous verrons que dans tous les tissus, dans tous les organes, c'est l'oxygène qui est toujours à la fois l'excitateur 
des phénomènes physico-chimiques et la condition de l'activité fonctionnelle de la matière organisée. L'oxygène pénètre dans les animaux par la surface respiratoire, et la circulation répand la vie dans tous les organes et dans tous les éléments organiques en leur distribuant l'oxygène dissous dans le sang artériel. C'est pourquoi le sang veineux ou sang privé d'oxygène amène la mort des éléments organiques, tandis que la transfusion du sang oxygéné est la seule transfusion vivifiante, ainsi que cela est connu depuis longtemps. Lorsqu'on injecte du sang oxygéné dans les tissus musculaires, nerveux, glandulaires, cérébraux, dont les propriétés vitales sont éteintes ou considérablement amoindries, on voit, sous l'influence de ce liquide oxygéné, chaque tissu reprendre ses propriétés vitales spéciales. Le muscle reprend sa contractilité; la motricité et la sensibilité reviennent dans les nerfs, et les facultés cérébrales reparaissent dans le cerveau. En injectant par exemple du sang oxygéné par la carotide dans la tête d'un chien décapité, on voit revenir peu à peu non-seulement les propriétés vitales des 
muscles, des glandes, des nerfs, mais on voit revenir également celles du cerveau; la tête reprend sa sensibilité, les glandes sécrètent, et l'animal exécute des mouvements de la face et des yeux qui paraissent dirigés par la volonté.

Quand, sous l'influence de l'oxygène, nous voyons revenir la contractilité dans un muscle, la motricité et la sensibilité dans les nerfs, cela ne nous semble pas surprenant; mais quand nous voyons que l'oxygène fait reparaître l'expression de l'intelligence dans le cerveau, l'expérience nous frappe toujours comme quelque chose de merveilleux et d'incompréhensible. C'est pourtant au fond toujours la même chose, et ce qui se passe pour le cerveau ne nous semble extraordinaire que parce que nous confondons les causes avec les conditions des phénomènes. Nous croyons à tort que le déterminisme dans la science mène à conclure que la matière engendre les phénomènes que ces propriétés manifestent, et cependant nous répugnons instinctivement à admettre que la matière puisse avoir par elle-même la faculté de penser, de sentir. En effet, dès que nous avons reconnu 
plus haut que la matière organisée est dépourvue de spontanéité comme la matière brute, elle ne peut pas plus qu'elle avoir conscience des phénomènes qu'elle présente.

Pour le physiologiste qui se fait une juste idée des phénomènes vitaux, le rétablissement de la vie et de l'intelligence dans une tête sous l'influence de la tranfusion du sang oxygéné n'a absolument rien d'anormal ou d'étonnant; c'est le contraire qui le surprendrait. En effet, le cerveau est un mécanisme conçu et organisé de façon à manifester les phénomènes intellectuels par l'ensemble d'un certain nombre de conditions. Or, si l'on enlève une de ces conditions (l'oxygène du sang par exemple), il est bien certain qu'on ne saurait concevoir que le mécanisme puisse continuer de fonctionner; mais si l'on restitue la circulation sanguine oxygénée avec les précautions exigées, telles qu'une température et une pression convenables, et avant que les éléments cérébraux soient altérés, il n'est pas moins nécessaire que le mécanisme cérébral reprenne ses fonctions normales. 
Les mécanismes vitaux, en tant que mécanismes, ne diffèrent pas res mécanismes non vitaux.

Si dans une horloge électrique, par exemple, on enlevait l'acide de la pile, on ne concevrait pás que le mécanisme continuât de marcher; mais, si l'on restituait ensuite convenablement l'acide supprimé, on ne comprendrait pas non plus que le mécanisme se refusàt à reprendre son mouvement. Cependant on ne se croirait pas obligé pour cela de conclure que la cause de la division du temps en heures, en minutes, en secondes, indiquées par l'horloge, réside dans les qualités de l'acide ou dans les propriétés du cuivre ou de la matière qui constitue les aiguilles et les rouages du mécanisme.

De même, si l'on voit l'intelligence revenir dans un cerveau et dans une physionomie auxquels on rend le sang oxygéné qui leur manquait pour fonctionner, on aurait tort d'y voir la preuve que la conscience et l'intelligence sont dans l’oxygène du sang ou dans la matière cérébrale.

Les mécanismes vitaux, ainsi que nous l'arons 
déjà dit, sont passifs comme les mécanismes non vitaux. Les uns et les autres ne font qu'exprimer ou manifester l'idée qui les a conçus et créés.

En résumé, nous n'avons à constater dans tout ce qui précède que les conditions d'un déterminisme physico-chimique nécessaire pour la manifestation des phénomènes vitaux aussi bién que pour la manifestation des phénomènes minéraux. Nous ne saurions done y chercher des explications qui aboutiraient à un matérialisme absurde ou vide de sens.

\section{III}

L'organisme animal n'est en réalité qu'ıne machine vivante qui fonctionne suivant les lois de la mécanique et de la physico-chimie ordinaires et à l'aide des procédés particuliers qui sont spéciaux aux instruments vitaux consti- 
tués par la matière organisée; mais les êtres vivants ont en outre pour caractère essentiel d'être périssables ou mortels. Ils doivent se renouveler et se succéder, car ils ne sont que les représentants passagers de la vie, qui est éternelle.

Il nous reste à parler maintenant des phénomènes de rénovation organique, qui ont toujours été considérés comme les phénomènes de la vie les plus mystérieux, par conséquent les plus irréductibles aux lois physico-chimiques et les plus difficiles à régir.

L'évolution d'un être nouveau, ainsi que sa nutrition, sont de véritables créations organiques qui s'accomplissent sous nos yeux. Toutefois ces phénomènes de création organique ne peuvent s'appliquer qu'à l'arrangement moléculaire matériel spécial qui caractérise la matière organisée, car les corps chimiques élémentaires qui composent la matière organisée sont absolument les mêmes que ceux qui forment la matière inorganique. Au point de vue chimique, la création de la matière vivante ne serail donc encore ici que le reflet des combinaisons mi- 
DE LA PHYSIOLOGIE GÉNÉRALE.

nérales sans nombre qui ont lieu dans le monde cosmique par suite d'arrangements moléculaires nouveaux et de mutations chimiques particulières qui s'opèrent incessamment autour de nous. Quant à la création primitive, elle nous échappe complétement dans tous les cas. Dans le monde tel que la science le connaît, rien ne se crée, rien ne se perd; il n'y a que des échanges et des transformations de matières et de forces qui se succèdent et s'équivalent d'une manière nécessaire et constante dans l'apparition des phénomènes de la nature.

Les corps vivants sont des composés instables qui se désorganisent sans cesse sous les influences cosmiques qui les entourent; ils ne vivent qu'à cette condition, et les organes formés par la matière vivante s'usent et se détruisent comme les organes formés par la matière inerte. Pour que la vie continue, il faut donc que la matière organisée qui forme les éléments histologiques se renouvelle constamment à mesure qu'elle se décompose, de sorte que l'on peut regarder la cause de la vie comme résidant véritablement dans la puissance d'organisation 
qui crée la machine vivante et répare ses pertes incessantes.

Les anciens physiologistes animistes et vitalistes avaient bien aperçu cette double face que représentent Jes phénomènes des êtres vivants. C'est pourquoi ils admettaient qu'un principe intérieur de la vie, qui était le principe créateur ou régénérateur, se trouvait en lutte avec les forces physico-chimiques extérieures qui constituent les agents destructeurs de l'organisme. Toutefois, si les influences physico-chimiques extérieures sont les causes de la mort ou de désorganisation de la matière vivante, cela ne veut pas dire, comme l'ont cru les vitalistes, qu'il y ait incompatibilité entre les phénomènes de la vie et les phénomènes physico-chimiques; il y a au contraire, comme nous l'avons vu, harmonie parfaite et nécessaire, car les causes qui détruisent la matière orģınisée sont celles qui la font vivre, c'est-à-dire manifester ses propriétés. Cela ne prouve pas davantage qu'il y ait combat ou lutte entre deux principes opposés, l'un de vie, qui résiste, l'autre de mort, qui attaque et finit toujours 
DE LA PHYSIOLOGIE GENERALE.

par être victorieux. En un mot, il n'y a pas dans les corps vivants deux ordres de forces séparées et opposées par la nature de leurs phénomènes, les unes qui créent la matière organisée avec ses propriétés caractéristiques, les autres qui la détruisent en la faisant servir aux manifestations vitales; il n’y a que des éléments histologiques qui fonctionnent évolutivement et tous suivant une même loi.

Nous savons qu'il y a des éléments musculaires, nerveux, glandulaires, qui servent aux manifestations des phénomènes de sensibilité, de mouvement, de sécrétion. Il y a de même des éléments ovariques et plasmatiques qui ont pour propriété de créer les êtres nouveaux et d'entretenir par la nutrition les mécanismes vitaux; mais ces éléments créateurs et nutritifs, comme les autres, s'usent et meurent en accomplissant leurs fonctions, qui donnent ellesmêmes les conditions d'une rénovation incessante. De même dans le jeu d'une machine inerte les ouvriers se fatiguent et dépensent aussi bien leurs forces, soit qu'ils travaillent à construire et à réparer les rouages de cette ma- 
chine, soit qu'ils travaillent à les faire fonctionner et à les user. Les phénomènes d'organogénèse ou de création organique ne sont donc ni plus ni moins mystérieux pour le physiologiste que tous les autres. Ils résident dans des éléments histologiques caractérisés, et ils ont leurs conditions physico-chimiques d'existence bien déterminées.

L'élément de création organique des êtres vivants est une cellule microscopique, l'ovule ou le germe. Cet élément est sans contredit le plus merveilleux de tous, car nous voyons qu'il a pour fonction de produire un organisme tout enlier.

On ne s'étonne plus des phénomènes qu'on a sans cesse sous les yeux; comme dit Montaigne, " l'habitude en ôte l'étrangeté. » Cependant qu'y a-t-il de plus extraordinaire que cette création organique à laquelle nous assistons, et comment pouvons-nous la rattacher à des propriétés inhérentes à la matière qui constitue l'ouf?

Quand la physiologie générale reut se rendre compte de la force musculaire par exemple, 
elle constate qu'une substance contractile vient agir directement en vertu des propriétés inhérentes à sa constitution physique ou chimique; mais, quand il s'agit d'une évolution organique qui est dans le futur, nous ne comprenons plus cette propriété de matière à longue portée. L'œuf est un devenir, il représente une sorte de formule organique qui résume l'être dont il procède et dont il a gardé en quelque sorte le souvenir évolutif.

Les phénomènes de création organique des ètres vivants me semblent bien de nature à dé. montrer une idée que j'ai déjà indiquée, à savoir que la matière n'engendre pas les phénomènes qu'elle manifeste. Elle n'est que le substratum et ne fait absolumen que donner aux phénomènes leurs conditions de manifestation, seul intermédiaire par lequel le physiologiste peut agir sur les phénomènes de la vie. C'est pourquoi ces conditions doivent être soumises à un déterminisme absolu et rigoureux, qui constitue le principe fondamental de toutes les sciences expérimentales. L'œuf ou le germe est un centre puissant d'action nutritive, et c'est à CLAUDE BERNARD. 
ce titre qu'il fournit les conditions pour la réalisation d'une idée créatrice qui se transmet par hérédité ou par tradition organique. L'œul', en présidant à la création de l'organisme, opère le renouvellement des êtres, et devient par suite la condition primordiale de tous les phénomènes ultérieurs de la vie.

Quand on observe l'évolution ou la création d'un être vivant dans l'œuf, on voit clairement que son organisation est la conséquence d'une loi organogénique qui préexiste d’après une idée préconcue, et qui s’est transmise par tradilion organique d'un être à l'autre. On pourrait trouver dans l'étude expérimentale des phénomènes d'histogenèse et d'organisation la justification des paroles de Gothe, qui compare la nature à un grand artiste. C'est qu'en effet la nature et l'artiste sembleät procéder de même dans la manifestation de l'idée créatrice de leur œuvre.

Nous voyons dans l'évolution apparaître une simple ébauche de l'être avant toute organisation. Les contours du corps et des organes sont d'abord simplement arrêtés, en commencant, bien entendu, par les échafaudages organiques 
provisoires qui serviront d'appareils fonctionnels temporaires au fœtus. Aucun tissu n'est d'abord distinct, toute la masse n'est constituée que par des cellules plasmatiques ou embryonnaires; nıais dans ce carıevas vital est tracé le dessin idéal d'une organisation encore invisible pour nous, qui a assigné d'avance à chaque partie, à chaque élément, sa place, sa structure et ses propriétés. Là où doirent être des vaisseaux sanguins, des nerfs, des muscles, des os, les cellules embryonnaires se changent en globules de sang, en tissus artériels, veineux, musculaires, nerveux et osseux. L'organisation ne se réalise pas d'emblée; d'abord vague et seulement ébauchée, elle ne se perfectionne que par différenciations élémentaires, c'est-à-dire par un fini dans le détail de plus en plus achevé.

Ce n'est pas tout : cette puissance créatrice ou organisatrice n'existe pas seulement au début de ia vie dans l'œuf, l'embryon ou le fœtus; elle poursuit son œuvre chez l'adulte, en présidant aux manifestations des phénomènes vitaux, car c'est elle qui entretient par la nutri- 
tion et renouvelle d'une manière incessante la matière et les propriétés des éléments organiques de la machine vivante. La nutrition n'est donc rien autre chose que cette puissance génératrice continuée et s'affaiblissant de plus en plus. C'est pourquoi il faut comprendre sous la dénomination de phénomènes organotrophiques tous les phénomènes d'organisation, de nutrition ou sécrétion organique chez l'embryon, le fœtus, l'adulte, parce qu'ils sont toujours soumis à une seule et même loi.

Les conditions physico-chimiques ambiantes règlent les manifestations vitales du germe ou de l'ovule comme celles de tous les autres éléments organiques.

Nous avons vu précédemment que la présence de l'oxygène provoque les manifestations des phénomènes de contraction dans les muscles, de motricité et de sensibilité dans les nerfs, d'intelligence dans le cerveau. L'oxygène conserve encore ici la même influence sur la manifestation de l'idée créatrice ou évolutive renfermée dans l'œuf. Si l'ovule ne recoit pas l'action directe ou indirecte de l'oxygène, l'évolu- 
tion ne peut avoir lieu. Quand l'incubation est intérieure, dans l'utérus, l'oxygène arrive par le sang; quand l'évolution est extérieure, l'oxygène arrive directement par l'air. Si l'on vernit un œuf de poule afin d'empêcher l'air de pénétrer par les pores de la coquille, l'ovule qu'il contient ne peut se développer et créer un être nouveau; de même, si l'on opère l'incubation de l'œuf d'oiseau dans un air confiné, l'évolution n'a lieu que quand l'oxygène existe dansl'air, et elle s'arrête, si l'on soustrait ce gaz du milieu d'incubation.

En résumé, nous voyons que le physiologiste, en s'adressant aux conditions de vitalité des divers éléments histologiques, a la possibilité d'exercer son empire sur tous les phénomènes vitaux, de quelque nature qu'ils soient.

La vie est une cause première qui nous échappe comme toutes les causes premières, et dont la science expérimentale n'a pas à se préoccuper; mais toutes les manifestations vitales, depuis la simple contraction musculaire jusqu'à l'expression de l'intelligence et à l'apparition de l'idée créatrice organique, ont chez. 
les êtres vivants des conditions physico-chimiques d'existence bien déterminées que nous pouvons saisir, et sur lesquelles nous pouvons agir pour régler les phénomènes auxquels président les éléments histologiques.

La physiologie a donc une base expérimentale tout aussi réelle et tout aussi solide que les sciences expérimentales des corps bruts. Son problème est sans doute très-complexe; mais, comme on le voit, elle ne rêve point une chimère en poursuivant la conquête de la nature vivante.

L'homme a entre les mains les instruments de sa puissance sur les êtres vivants. Il en acquiert chaque jour la preuve en voyant les actions toxiques et médicamenteuses si variées qu'il provoque dans l'organisme '. La physiologie nous apprend que les poisons et les médicaments ne sont actifs que parce qu'ils pénètrent dans le sang, c'est-à-dire dans le milieu intérieur où vivent les éléments organiques.

D’un autre côté, la vitalité des éléments ne

1. Cl. Bernard, Leçons sur les effets des substanies toxiques et médicamenteuses. Paris, 1857. 
DE LA PHYSIOLOGIE GÉNÉRALE.

peut être modifiée qu'autant que la substance active produit autour d'eux des modifications physico-chimiques déterminées, d'où il suit que le problème du physiologiste consiste à connaître quelles sont les modifications physicochimiques spéciales qui favorisent, troublent ou détruisent les propriétés des divers éléments histologiques; mais, outre les actions immédiates 'produites par les agents modificateurs énergiques, poisons ou médicaments, le physiologiste peut encore exercer une action profonde et durable sur les organismes vivants en modifiant les éléments histologiques au moyen de la nutrition.

Ón produit par la nutrition ou par la culture des modifications considérables et bien connues dans les organismes végétaux. On crée ainsi des variétés dans l'espèce, et même des espèces nouvelles. Chez les animaux il en est de même, et nous savons, par exemple, que la production de la sexualité et beaucoup d'autres modifications organiques importantes se réduisent à des questions d'alimentation et de nutrition embryonnaire. 
Les éléments histologiques ne suivent la tradition organique des êtres dont ils procèdent qu'autant qu'ils se trouvent placés dans des conditions convenables de nutrition. Une simple cellule animale ou végétale qui, dans certaines circonstances, peut rester indifférente prend un développement nouveau, si l'on vient à changer les conditions nutritives. En modifiant les milieux intérieurs nutritifs, et en prenant la matière organisée en quelque sorte à l'état naissant, on peut espérer changer sa direction évolutive et par conséquent son expression orgarique finale.

En un mot, rien ne s'oppose à ce que nous puissions ainsi produire de nouvelles espèces organisées, de même que nous créons de nouvelles espèces minérales, c'est-à-dire que nous ferions apparaître des formes organisées qui existent virtuellement dans les lois organogéniques, mais que la nature n'a point encore réalisées. 


\section{IV}

Jusqu'à présent, toutes les actions modificatrices de l'homme sur l'organisation des êtres vivants sont encore très-bornées, et ne sont que l'œuvre d'un grossier empirisme. Ici comme partout, c'est l'observation empirique qui doit nous tracer la route scientifique. La science commence seulement à pénétrer dans l'étude des phénomènes de la vie; mais elle marche dans une voie qui lui permettra certainement d'éclairer avec le temps toutes les obscurités qui couvrent maintenant les divers problèmes de la physiologie générale.

La physiologie est destinée à servir de base à toutes les sciences qui veulent arriver à régir les phénomènes de la nature vivante; ces sciences intéressent par conséquent l'humanité au plus haut degré. 
L'agriculture ne saurait se fonder sur les seules sciences naturelles. Elle s'appuie nécessairement sur les sciences expérimentales, sur la physique et la chimie d'un côté et sur la physiologie animale et végétale de l'autre.

L'hygiène et la médecine d'obscrvation, fondées par Hippocrate depuis vingt-trois siècles, ne pourront donner naissance à la médecine expérimentale et sortir de l'empirisme que lorsque la physiologie expérimentale leur fournira le point d'appui qui leur manque.

La physiologie est donc une science nouvelle sur laquelle on doit fonder les plus légitimes espérances, et que l'on doit protéger et développer le plus possible.

Tout ce que nous avons dit en commencant sur la nécessité de séparer dans les sciences biologiques le problème des sciences naturelles du problème des sciences expérimentales, ne se rapporte point seulement à une distinction purement théorique qu'il convient de faire entre la physiologie d'une part, la zoologie et la phytologie ou botanique de l'autre; il s'agit encore d'une séparation pratique [qu'il faut établir 
entre ces sciences et qui est destinée à exercer la plus grande influence sur leurs progrès réciproques.

Les sciences procèdent analytiquement dans leur développement; c'est pourquoi il s'estétabli successivement des divisions et des subdivisions scientifiques qui continuent encore; mais en se divisant et en se subdivisant, les sciences ne font que s'accroître et s'épanouir en des problèmes nouveaux qui s'engendrent les uns les autres sans se confondre ni s'amoindrir. Le problème des sciences naturelles biologiques ne perdra rien de son importance en se sépirant du problème des sciences expérimentales physiologiques. Au contraire, les deux ordres de sciences ne s'en développeront que plus librement et avec plus d'éclat; mais la physiologie expérimentale, constituant un plus jeune rameau de l'arbre scientifique, tire nécessairement la séve du tronc et des branches inférieures des sciences biologiques : d'où il suit que les progrès particuliers de cette dernière science doivent être considérés non-seulement comme des résultats dus à la culture d'une 
science distincte, mais encore comme le fruit de l'érolution totale des autres sciences biologiques.

La physiologie expérimentale, ayaut son problème spécial, constitue une science expérimentale autonome qui, dans l'ordre des sciences biologiques, est tout aussi distincte et indépendante de la zoologie et de la botanique que la chimie, dans l'ordre des sciences minérales, est indépendante de la géologie et de la minéralogie. Dès lors la physiologie expérimentale doit posséder ses moyens particuliers de travail scientifique, séparés de ceux de la zoologie et de la botanique. C'est là un point capital dans la question qui nous occupe.

Un des obstacles que la physiologie expérimentale a dû rencontrer nécessairement dans son évolution, c'est l'antagonisme des naturalistes, - zoologistes, botanistes, anatomistes, - qui, pensant que la physiologie rentrait dans leur domaine et leur appartenait, réclamaient pour leurs musées et leurs collections toutes les améliorations à faire dans les sciences biologiques, et s'opposaient à la création 
de laboratoires indépendants et de chaires spéciales de physiologie. C'est une loi commune dans les sciences comme dans toutes les insti. tutions humaines que le progrès ne se fasse que par la lutte ou toutau moins à la suite d'efforts longtemps répétés; mais aujourd'hui la physiologie a conquis l'indépendance scientifique, et les conséquences de cette conquête se font sentir chaque jour de plus en plus dans l'organisation de son enseignement. On sépare maintenant l'enseignement de la zoologie et de l'anatomie de celui de la physiologie, et de grands et beaux laboratoires de physiologie expérimentale, sous le nom d'instituts physiologiques ${ }^{1}$, s'élèvent à côté des musées des zoologistes el

1. A Heidelberg, la chaire d'anatomie et de physiologie a èté divisée, et le bel institut physiologique de M. Helmholtz a été créé. A Berlin, la chaire d'anatomie, zoologie, physiologie, de J. Muller, a été partagée : M. Reichert a étẻ chargẻ de la zoologie et anatomie, et M. Du Bois-Reymond de la physiologie. A Würtzbourg, la chaire d'anatomie et physiologie a été également divisée. A Upsal, on a opéré dernièrement la mème séparation, et on a créé une chaire de physiologie. Ces exemples sont suivis dans beaucoup d'autres universités. 
des botanistes, comme les laboratoires de chimie et de physique se sont élevés à côté des musées du géologue et du minéralogiste.

la France a marché en avant dans linitiation aux découvertes et aux idées qui ont provoqué la rénovation de la physiologie expérimentale moderne, mais il reste des réformes à faire pour installer cet enseignement. Partout la physiolog̣ie expérimentale est appréciée et accueillie comme la science moderne qui monte à l'horizon et à laquelle est réservé le plus brillant avenir. Elle a des laboratoires spéciaux et des chaires séparées qui se muliiplient de plus en plus dans les universiles de la Russie, de l'Allemagne, de la Suède, de la Hollande, de la Belgique, de l'Italie. Des instituts sont déjà créés à Pétersbourg, à Heidelberg et ailleurs; il s'éleve à Leipzigg un magnifique institut plıysiologique qui sera sous la direction de l'éminent professeur Ludwig.

Toutes les nations rivalisent en quelque sorte dans l'empressement qu'elles mettent à protéger la physiologie et à lui fournir tous las moyens de culture qui lui sont nécessaires. 
Je n'ai plus qu'un vou à exprimer, c'est que notre pays, ainsi que je l'ai déjà dit, qui a eu la gloire de donner le jour à d'illustres promoteurs de la physiolorie moderne ${ }^{1}$, s'associe au mouvement scientifique général et encourage les sciences physiologiques, dont il est important de faciliter l'accès aux jeunes générations de savants.

Mais la physiologie ne saurait borner son rôle à expliquer les fonctions les plus grossières du corps humain; elle doit éclairer aussi les mécanismes de la psychologie, elle est appelée par conséquent à réagir directement sur les opinions philosophiques. Peut-être se rencontrera-t-il des esprits qui, poursuivant à l'aide de la logique les conséquences extrêmes de ce que nous avons dit sur la possibilité de réğler tous les phénomènes de la vie, seront porlés à voir dans cette prétention physiologique une contradiction avec la philosophie et même une négation de la liberté. De semblables opposi-

(1) Voyez mon Rapport au ministre de l'instruction publique sur la marche et les progrès de la physiologie générale en France. 
148 PROBLÈME DE LA PHYSIOLOGIE GÉNÉRALE.

tions ne me paraissent pas à craindre, car lia science ne saurait détruire les faits évidents d'eux-mêmes, seulement elle peut arriver à les comprendre autrement. Je me bornerai à dire, par exemple, que le déterminisme absolu que le physiologiste reconnaît et démontre dans les phénomènes de la vie est lui-même une condition nécessaire de la liberté. Le savant ne concevrait pas en effet qu'un phénomène, quel qu'il soit, puisse être librement manifesté dès qu'il n'est régi par aucune loi et qu'il est indéterminé par nature. Je pense d'ailleurs qu'il n'y a pas pour le moment à se préoccuper de semblables questions. Nous n'avons qu’à continuer nos investigations et à attendre patiemment les solutions de la science. Elle ne peut nous conduire qu'à la vérité, et tenons pour certain que la vérité scientifique sera toujours plus belle que les créations de notre imagination et que les illusions de notre ignorance. 


\section{DÉFINITION DE LA VIE}

LES THÉORIES ANGIENNES ET LA SGIENGE MODERNE

I

Dès la plus haute antiquité, des philosophes ou des médecins célèbres ont regardé les phénomènes qui se déroulent dans les êtres vivants comme émanés d'un principe supérieur et immatériel agissant sur la matière inerte et obéissante. Telle est la pensée de Pythagore, de Platon, d'Aristote, d'Hippocrate ', acceptée plus

1. Hippocrate, Euvres complètes, trad. Littré. Paris, 1840. 
tard par les philosophes et les savants mystiques du moyen âge, Paracelse, Van Helmont, et par les scolastiques. Cette conception atteignit dans le cours du dix-huitième siècle son apogée de faveur et d'influence avec le célèbre médecin Stahl, qui lui donna une forme plus nette en créant l'animisme. L'animisme a été l'expression outrée de la spiritualité de la vie; Stahl fut le partisan déterminé et le plus dogmatique de ces idées perpéluées depuis Aristote. On peut ajouter qu'il en fut le dernier représentant; l'esprit moderne n'a pas accueilli une doctrine dont la contradiction avec la science était devenue trop manifeste.

D’un autre côté, et par opposition aux idées qui précèdent, nous voyons, avant même que la physique et la chimie fussent constitıées, et que l'on connût les phénomènes de la matière brute, les tendances philosophiques, en avancant sur les faits, essayer d'établir l'identité entre les phénomènes des corps inorganiques et ceux des corps vivants. Cette conception est le fond de l'atomisme de Démocrite et d'Épicure. Les atomistes ne reconnaissent pas d'intelli- 
gence motrice. Le monde se meut par lui-même éternellement. Ils ne considèrent qu'une seule espèce de matière, dont les éléments, grâce à leurs figures, jouissent de la propriété de former, en s'attachant les uns aux autres, les combinaisons les plus diverses, et de constituer les corps inorganiques et sans vie, aussi bien que les êtres organisés qui vivent et sentent comme les animaux, qui sont raisonnables et libres comme l'homme.

Cette seconde hypothèse affecta ainsi dès son début une forme exclusivement matérialiste; mais, chose remarquable, les philosophes les plus convaincus de la spiritualité de l'âme, tels que Descartes et Leibniz, ne devaient pas tarder d'adopter une facon de voir analogue qui attribuait au jeu des forces brutes toutes les manifestations saisissables de l'activité vitale. La raison de cette apparente contradiction réside dans la séparation presque absolue qu'ils établirent entre l'âme et le corps. Descartes a donné une définition métaphysique de l'âme et une définition physique de la vie. L'âme est le principe supérieur qui se manifeste par la pen- 
séc, la vie n'est qu’un effet supérieur des lois de la mécanique. Le corps humain est une machine formée de ressorts, de leviers, de canaux, de filtres, de cribles, de pressoirs. Cettemachine est faite pour elle même; l'âme s'y ajoute pour contempler en simple spectatrice ce qui se passe dans le corps, mais elle n'intervient en rien dans le fonctionnement vital. Les idées de Leibniz, au point de vue physiologique, ont beaucoup d'analogie avec celles de Descartes. Comme lui, i] sépare l'âme du corps, et, quoiqu'il admette entre eux une concordance préétablie par Dieu, il leur refuse toute espèce d'action réciproque. “Le corps, dit-il, se développe mécaniquement, et les lois mécaniques ne sont jamais violées dans les mouvements naturels; tout se fait dans les âmes comme s'il n'y avait pas de corps, et tout se fait dans le corps comme s'il n'y avait pas d'âme. »

Stahl comprit tout autrement la nature des phénomènes de la vie et les rapports de l'âme et du corps. Dans les actes vitaux, il rejette toutes les explications qui leur seraient communes avec les phénomènes mécaniques, physiques et 
chimiques de la matière brute. Célèbre chimiste lui-mème, il combat avec beaucoup de puissance et d'autorité surtout les exagérations des médecins-chimistes ou iatro-chimistes, tels que Sylvius de LeBoë, Willis, etc., qui expliquaient tous les phénomènes de la vie par des actions chimiques : fermentations, alcalinités, acidités, effervescences. Il soutient que non-seulement les forces chimiques sont différentes des forces qui régissent les phénomènes de la vie, mais qu'elles sont en antagonisme avec elles, et qu'elles tendent à détruire le corps vivant au lieu de le conserver. Il faut donc, suivant Stahl, une force vitale qui conserve le corps contre l'action des forces chimiques extérieures qui tendent sans cesse à l'envahir et à le détruire; la vie est le triomphe de celles-ci sur celles-lá. Par ces idées, Stahl fonda le vitalisme; mais il ne s'arrêta pas à ce terme : ce n'était qu'un premier pas dans la voie qui devait le conduire à l'animisme. Cette force vitale, dit-il, qui sans cesse lutte contre les forces physiques, agit avec intelligence, dans un dessein calculé, pour la conservation de l'organisme. Or, si la force 
vitale est intelligente, pourquoi la distinguer de l'âme raisonnable?

Basile Valentin et son disciple Paracelse avaient multiplié sans mesure l'existence de principes immatériels intelligents, les archées, qui réglaient les phénomènes du corps vivant. Van Helmont, le plus célèbre représentant de ces doctrines archéiques, qui allia avec le génie expérimental l’imagination la plus déréglée dans ses écarts, avait conçu toute une hiérarchie de ces principes immatériels. Au premier rang se trouvait l'àme raisonnable et immortelle se confondant en Dieu, ensuite l'âme sensitive et mortelle, ayant pour agent un autre archée principal, qui lui-même commandait ì une foule d'archées subalternes, les blıs.

Stahl, qui à un siècle de distance est le continuateur de Van Helmont, simplifie toutes ces conceptions de principes intelligents, d'esprits recteurs ou d'archées. Il n'admet qu'une seule âme, l'âme immortelle, chargée en même temps du gouvernement corporel. L’âme est pour lui le principe même de la vie. La vie est un des modes de fonctionñement de l'âme, c'est 
son acte vivifique. L'âme immortelle, force intelligente et raisonnable, gouverne directement li matière du corps, le met en œuvre, la dirige vers sa fin. C'est elle qui non-seulement dicte nos actes volontaires, mais c'est elle qui fait battre le cœur, circuler le sang, respirer le poumon, sécréter les glandes. Si l'harmonie de ces phénomènes est troublée, si la maladie survient, c'est que l'âme n'a pas rempli ces fonctions, ou n'a pu résister efficacement aux causes extérieures de destruction. Une semblable doctrine avait quelque chose d'étrange et de contradictoire, car l'action d'une âme raisonnable sur les actes vitaux semble supposer une direction consciente, et l'observation la plus simple nous apprend que toutes les fonctions de nutrition, -- circulation, sécrétions, diges tion, etc., - sont inconscientss et involontaires, comme si, selon l'expression d'un physiologiste philosophe, la nature avait voulu par prudence soustraire ces importants phénomènes aux caprices d'une volonté ignorante. L'animisme de Stahl était done empreint d'une exagéé ration qui porta ses successeurs, sinon it 
l'abandonner, au moins à le modifier profondément.

Les idées de Descartes et celles. de Stahl avaient fait dans la science une impression profonde et créé deux courants qui devaient arriverjusqu'à nous.

Descartes avait posé les premiers principes et appliqué les lois mécaniques au jeu de la machine du corps de l'homme; ses adeptes étendirent et précisèrent les explications mécaniques des divers phénomènes vitaux. Parmi les plus célèbres de ces iatro-mécaniciens, il faut citer au premier rang Borelli, ensuite Pitcairn, Hales, Keil, surtout Boerhaave, dont l'influence fut prépondérante. De son côté, l'iatro-chimie, qui n'est qu'une face de la doctrine cartésienne, poursuivit sa marche et fut définitivement fondée à l'avénement de la chimie moderne. Descartes et Leibniz avaient pesé en principe que partout les lois de la mécanique sont identiques; qu'il n'y a pas deux mécaniques, l'une pour les corps bruts, l'autre pour les corps vivants.

A la fin du siècle dernier, Lavoisier et La- 
place vinrent démontrer qu'il n'y a pas non plus deux chimies, l'une pour les corps bruts, l'autre pour les êtres vivants. Ils prouvèrent expérimentalement que la respiration et la production de chaleur ont lieu dans le corps de l'homme et des animaux par des phénomènes de combustion tout à fait semblables à ceux qui se produisent pendant la calcination des métaux.

C'est vers la même époque que Bordeu, Barthez, Grimaud, brillaient dans l'école de Montpellier. Ils étaient les successeurs de Stahl; néanmoins ils ne conservèrent que la première partie de la doctrine du maître, le vitalisme, et en répudièrent la seconde, l'animisme. Contrairement à Stahl, ils veulent que le principe de la vie soit distinct de l'âme; mais avec lui ils admettent une force vitale, un principe vital recteur dont l'unité donne la raison de l'harmonie des manifestations vitales, et qui agit en dehors des lois de la mécanique, de la physique et de la chimie.

Cependant le vitalisme se modifia peu à peu dans sa forme; la doctrine des propriétés vitales 
marqua une époque importante dans l'histoire de la physiologie. Au lieu de conceptions métaphysiques qui avaient régné jusque-là, voici une conception physiologique qui cherche à expliquer les manifestations vitales par les propriétés mêmes de la matière des tissus ou des organes.

Déjà à la fin du dix-septième siècle Glisson avait désigné l'irritabilité comme cause immédiate de mouvements de la fibre vivante. Bordeu, Grimaud et Barthez avaient entrevu plus ou moins vaguement la même idée. Haller attacha son nom à la découverte de cette faculté motrice en nous faisant connaître ses mémorables expériences sur l'irritabilité et la sensibilité des diverses parties du corps.

Toutefois c'est seulement au commencement de ce siècle que Xavier Bichat, par une illumination du génie, comprit que la raison des phénomènes vitaux devait être cherchée non pas dans un principe d’ordre supérieur immatériel, mais au contraire dans les propriétés de la matière, au sein de laquelle s'accomplissent ces phénomènes. Sans doute Bichat n'a pas défini 
les propriétés vitales, il leur donne des caractères vagues et obscurs; son génie, comme il arrive souvent, n'est pas d'avoir découvert les faits, c'est d'en avoir compris le sens en émettant le premier cette idée générale, lumineuse et féconde, qu'en physiologie comme en physique les phénomènes doivent être rattachés à des propriétés comme à leur cause. "Le rapport des propriétés comme causes avec les phénomènes comme effets, dit-il ${ }^{1}$, est un axiome presque fastidieux à répéter aujourd'hui en physique et en chimie; si mon livre établit un axiome analogue dans les sciences physiologiques, il aura rempli son but. "Puis, continuant, il ajoute : "Il y a dans la nature deux classes d'êtres, deux classes de propriétés, deux classes de sciences. Les êtres sont orgraniques ou inorganiques; les propriétés sont vitales ou non vitales, les sciences sont physiques ou physiologiques.... »

Il importe ici et dès l'abord de bien comprendre la pensée de Bichat. On pourrait croire

1. Bichat. Anatomie générale. Préface. 
qu'il va se rapprocher des physiciens et des chimistes, puisqu'il place comme eux la cause des phénomènes dans les propriétés de la matière; c'est le contraire qui arrive, et Bichat s'en éloigne et s'en sépare d'une manière aussi complète que possible. En effet, le but poursuivi dans tous les temps par les iatro-mécaniciens, physiciens ou chimistes, a été d'établir une ressemblance, une identité entre les phé. nomènes des corps vivants et ceux des corps inorganiques. A l'encontre de ceux-ci, Bichat pose en principe que les propriétés vitales sont absolument opposées aux propriétés physiques, de sorte qu'au lieu de passer dans le camp des physiciens et des chimistes, il reste vitaliste avec Stahl et l'école de Montpellier. Comme eux, il considère que la vie est une lutte entre des actions opposées; il admet que les propriétés vitales conservent le corps vivant en entravant les propriétés physiques qui tendent à le détruire. Quand la mort survient, ce n'est que le triomphe des propriétés physiques sur leurs antagonistes. Bichat d'ailleurs résume complétement ses idées dans la définition qu'il donne 
de la vie : la vie esl l'ensemble des fonclions qui résistent à la mort, ce qui signifie en d'autres termes : la vie est l'ensemble des propriétés vitales qui résistent aux propriétés physiques.

Cette vue qui consiste à considérer les propriétés vitales comme des espèces d'entités métaphysiques qu'on ne définit pas clairement, mais qu'on oppose aux propriétés physiques ordinaires, a entraîné sans doute la recherche dans les mêmes erreurs que les autres théories vitalistes. Cependant la conception de Bichat, dégagée des erreurs presque inévitables à son époque, n'en reste pas moins une conception de génie sur laquelle s'est fondée la physiologie moderne. Avant lui, les doctrines philosophiques, animistes ou vitalistes, planaient de trop haut et de trop loin sur la réalité pour pouvoir devenir les initiatrices fécondes de la science de la vie; elles n'étaient capables que de l'engourdir en jouant le rôle de ces sophismes paresseux qui régnaient jadis dans l'école. Bichat, au contraire, en décentralisant la vie, en l'incarnant dans les tissus, et en rattachant ses manifestations aux propriétés de ces mêmes 
tissus, les a, si l'on veut, placés sous la dépendance d'un principe encore métaphysique, mais moins élevé en dignité philosophique, et pouvant devenir une base scientifique plus accessible à l'esprit de recherche et de progrès. Bichat, en un mot, s'est trompé, comme les vitalistes ses prédécesseurs, sur la théorie de la vie; mais il ne s'est pas trompé sur la méthode physiologique. C'est sa gloire de l'avoir fondée en placant dans les propriétés des tissus et des organes les causes immédiates des phénomènes de la vie.

Les idées de Bichat produisirent en physiologie et en médecine une révolution profonde et universelle. L'école anatomique en sortit, poursuivant avec ardeur dans les propriétés vitales des tissus sains et altérés l'explication des phénomènes de la santé et de la maladie. D’un autre côté les progrès des méthodes physiques, les découvertes brillantes de la chimie moderne, jetant une vive lumière sur les fonctions vitales, venaient chaque jour protester contre la séparation et l'opposition radicales que Bichat, ainsi que les vitalistes, avaient cru voir entre 
les phénomènes organiques et les phẻnomènes inorganiques de la nature.

C'est ainsi que nous trouvons encore près de nous dans Bichat et dans Lavoisier les représentants des deux grandes tendances philosophiques opposées que nous avons démêlées dès l'antiquité, à l'origine même de la science, l'une cherchant à réduire les phénomènes de la vie aux lois de la chimie, de la physique, de la mécanique, l'autre voulant au contraire les distinguer et les placer sous la dépendance d'un principe particulier, d'une puissance spéciale, quel que soit le nom qu'on lui donne, d'âme, d'urchée, de psyché, de méliateur plastique, d'esprit recteur, de force vitale ou de propriétés vitales. Cette lutte, déjà si vieille, n'est done pas encore finie; mais comment devrat-elle finir? L'une des doctrines arrivera-t-elle à triompher de l'autre et à dominer sans partage? Je ne le pense pas. Les progrès des sciences ont pour résultat d'affaiblir graduellement, et dans une égale mesure, ces premières conceptions exclusives nées de notre ignorance. L'inconnu faisant seul leur force, à mesure 
qu'il disparaît, les luttes doivent cesser, les doctrines opposées s'évanouir, et la vérité scientifique qui les remplace régner sans rivale.

\section{II}

Nous pouvons dire de Bichat, comme de la plupart des grands promoteurs de la science, qu'il a eu le mérite de trouver la formule pour les conceptions flottantes de son temps. Toutes les idées de ses contemporains sur la vie, toutes leurs tentatives pour la définir ne sont en quelque sorte que l'écho ou la paraphrase de sa doctrine.

Un chirurgien de l'Hôtel-Dieu de Paris, $\mathrm{Ph}$. J. Pelletan, enseigne que la vie est la résistance opposée par la matière organisée aux causes qui tendent sans cesse à la détruire.

Cuvier lui-même développe la même pensée, que la vie est une force qui résiste aux lois qui 
régissent la matière brute; la mort ne serait que le retour de la matière vivante sous l'empire de ces lois. Ce qui distingue le cadavre du corps vivant, e'est ce principe de résistance qui soutient ou qui abandonne la matière organisée, et pour donner une forme plus saisissante à son idée, Cuvier nous représente le corps d'une femme dans l'éclat de la jeunesse et de la santé subitement atteinte par la mort.

"Voyez, dit-il, ces formes arrondies et voluptueuses, cette souplêsse gracieuse des mouvements, cette douce chaleur, ces joues teintes de rose, ces yeux brillants de l'étincelle de l'amour ou du feu du génie, cette physionomie égayée par les saillies de l'esprit ou animée par le feu des passions; tout semble se réunir pour en faire un être enchanteur. Un instant suffit pour détruire ce prestige : souvent, sans cause apparente, le mouvement et le sentiment viennent à cesser, le corps perd sa chaleur, les muscles s'affaissent et laissent paraître les saillies anguleuses des os; les yeux devienneni ternes, les joues et les lèvres livides. Ce ne soni là que les préludes de changements plus horri- 
bles : les chairs passent au bleu, au vert, au noir; elles attirent l'humidité, et pendant qu'une portion s'évapore en émanations infectes, une autre s'écoule en sanie putride qui ne tarde pas à se dissiper aussi; en un mot, au bout d'un petit nombre de jours, il ne reste plus que quelques principes terreux et salins; les autres éléments se sont dispersés dans les airs et dans les eaux pour entrer dans d'autres combinaisons."

" Il est clair, ajoute Cuvier, que cette séparation est l'effet naturel de l'action de l'air, de l'humidité, de la chaleur, en un mot, de tous les agents extérieurs sur le corps mort, et qu'elle a sa cause dans l'attraction élective des divers agents pour les éléments qui le composaient. Cependant ce corps en était également entouré pendant la vic; leurs affinités pour ses molécules étaient les mêmes, et celles-ci y eussent cédé également, si elles n’avaient été retenues ensemble par une force supérieure à ces affinités, qui n'a cessé d'agir sur elles qu'à l'instant de la mort. "

Ces idées de contraste et d'opposition entre 
les forces vitales et les forces extérieures physico-chimiques, que nous retrouvons dans la doctrine des propriétés vitales, avaient déjà été exprimées par Stahl, mais en un langage obscur et presque barbare; exposées par Bichat arec une lumineuse simplicité et un grand charme de style, ces mêmes idées séduisirent et entraînèrent ious les esprits.

Bichat ne se contente point d'affirmer l'antagonisme des deux ordres de propriété qui se partagent la nature; mais en les caractérisant les unes et les autres il les oppose d'une manière saisissante.

"Les propriétés physiques des corps, dit-il, sont éternelles. A la création, ces propriétés s'emparèrent de la matière, qui en restera constamment pénétrée dans l'immense série des siècles. Les propriétés vitales sont au contraire essentiellement temporaires; la matière brute en passant par les corps vivants s'y pénètre de ces propriétés qui se trouvent alors unies aux propriétés physiques; mais ce n'est pas là une alliance durable, car il esi de la nature des propriétés vitales de s'épuiser; le temps les use 
dans le même corps. Exaltées dans le premier âge, restées comme stationnaires dans l'âge adulte, elles s'affaiblissent et deviennent nulles dans les derniers temps. On dit que Prométhée, ayant formé quelques statues d'hommes, déroba le feu du ciel pour les animer. Ce feu est l'emblème des propriétés vitales : tant qu'il brûle la vie se soutient; elle s'anéantit quand il s'éteint. ")

C'est uniquement de ce contraste dans la nature et dans la durée des propriétés physiques et des propriétés vitales que Bichat déduit tous les caractères distinctifs des êtres vivants et des corps bruts, toutes les différences entre les sciences qui les étudient.

Les propriétés physiques étant éternelles, dit-il, les corps bruts n'ont ni commencement ni fin nécessaires, ni âge, ni évolution; ils n’ont de limites que celles que le hasard leur assigne.

Les propriétés vitales étant au contraire changeantes el d'une durée limitée, les corps vivants sont mobiles et périssables; ils ont un commencement, une naissance, une mort, des âges, 
en un mol, une évolution qu'ils doivent parcourir. Les propriétés vitales se trouvant constamment en lutte avec les propriétés physiques, Je corps vivant, théâtre de celte lutte, en subit les alternatives. La maladie et la santé ne sont autre chose que les péripéties de ce combat : si les propriétés physiques triomphent définitivement, la mort en est la conséquence; si au contraire les propriétés vitales reprennent leur empire, l'être vivant guérit de sa maladie, cicatrise ses plaies, répare son organisme et rentre dans l'harmonie de ses fonctions. Dans les corps bruts rien de semblable ne s'observe; ces corps restent immuables comme la mort dont ils sont l’image.

De là une distinction profonde entre les sciences qu'il nomme vitales et celles qu'il appelle non vilales. Les propriétés physico-chimiques étant fixes, constantes, les lois des sciences qui en traitent sont également constantes et invariables; on peut les prévoir, les calculer avec certitude. Les propriétés vitales ayant pour caractère essentiel l'instabilité, toutes les fonctions vitales étant susceptibles d'urie foule de variétés, 
on ne peut rien prévoir, rien calculer dans leurs phénomènes.

D'où il faut conclure, dit Bichat, "que des lois absolument différentes président à l'une et à l'autre classe de phénomènes. ”

Telle est, dans ses grands traits et avec ses conséquences, la doctrine des propriétés vilales, qui a longtemps dominé dans l'école malgré les justes critiques dont elle est passible.

Nous allons examiner brièvement si la division des phénomènes en deux grands groupes, telle que l'établit la doctrine dont Bichat s'est fait l'éloquent défenseur, est bien fondée, et si elle ne serait pas plutôt une conception systématique que l'expression de la vérité.

D’abord est-il vrai que les corps de la nature inorganique soient éternels et que les corps vivants seuls soient périssables; n'y aurait-il pas entre eux de simples différences de degrés qui nous font illusion par leur grande disproportion?

Il est cerlain, par exemple, que la vie d'un éléphant peut paraître l'éternité par rapport à la vie d'un éphémère, et quand nous considérons 
la vie de l'homme relativement à la durée du milieu cosmique qu'il habite, elle doit nous paraître un instant dans l'infini du temps. Les anciens ont pensé ainsi : ils opposaient le monde vivant, où toui est sujet au changement et à la mort, au monde sidéral, immuable et incorruptible. Cette doctrine de l'incorruptibilité des cieux a régné jusqu'au dix-septième siècle. Les premières lunettes permirent alors de constater l'apparition d'une nouvelie étoile dans la constellation du Serpentaire; ce changement dans le ciel, accompli pour ainsi dire sous les yeux de l'observateur, commença d'ébranler la croyance des anciens : materiam coli esse inalterabilem. Aujourd'hui l'esprit des astronomes est familiarisé avec l'idée d'une mobilité et d'une évolution continuelle du monde sidéral. "Les astres n'ont pas toujours existé, dit M. Faye; ils ont eu une période de formation; ils auront pareillement une période de déclin, suivie d'une extinction finale. »

L'éternité des corps sidéraux invoquée par Bichat n'est donc pas réelle; ils ont une évolution comme les corps vivants, évolution lente, 
si on la compare à notre vie pressée, évolution qui embrasse une durée hors de proportion avec celle que nous sommes habitués à considérer autour de nous. D’un autre côté, les astronomes, avant de connaître les lois des mouvements des corps célestes, avaient imaginé des puissances, des forces sidérales, comme les physiologistes reconnaissaient des forces et des puissances vitales. Kepler lui-même admettait un esprit recteur sidéral par l'influence duquel « les planètes suivent dans l'espace des courbes savantes sans heurter les astres qui fournissent d'autres carrières, sans troubler l'harmonie réglée par le divin géomètre. »

Si les corps vivants ne sont pas seuls soumis à la loi d'évolution, la faculté de se régénérer, de se cicatriser, ne leur est pas non plus exclusive, quoique ce soit sur eux qu'elle se manisfeste plus activement.

Chacun sait qu'un organisme vivant, quand il a été mutilé, tend à se reiaire suivant les lois de sa morphologie spéciale : la blessure se cicatrise dans l'animal et dans la plante, la perle de substance se comble, et l'être se rétablit dans 
sa forme et son unité. Ce phénomène de reconstitution, de rédintégration, a profondément frappé les philosophes naturalistes, et ils ont beaucoup insisté sur cette tendance de la vie à l'individualité, qui fait de l'être vivant un tout harmonique, une sorte de petit monde dans le grand. Quand l'harmonie de l'édifice organique est troublée, elle tend à se rétablir.

Mais il n'est pas nécessaire d'invoquer, pour expliquer ces faits, une force, une propriété vitale en contradiction avec la physique. Les corps minéraux en effet se montrent doués de cette même unité morphologique, de cette même tendance à la rétablir. Les cristaux comme les êtres vivants ont leurs formes, leur plan particulier, et ils sont susceptibles d'éprouver les actions perturbatrices du milieu ambiant. La force physique qui range les particules eristallines suivant les lois d'une savante géométrie a des résultats analogues à celle qui range la substance organisée sous la forme d'un animal ou d'une plante.

M. Pasteur a signalé des faits de cicatrisation, de rédintégration cristalline, qui méritent 
toute notre attention. Il étudia certains cristaux et les soumit à des mutilations qu'il a vues se réparer très-rapidement et très-régulièrement. Il résuĩte de l'ensemble de ses recherches que, “ lorsqu'un cristal a été brisé sur l'une quelconque de ses parties et qu'on le replace dans son eau-mère, on voit, en même temps que le cristal s'agrandit dans tous les sens par un dépôt de particules cristallines, un travail trèsactif avoir lieu sur la partie brisée ou déformée, et en quelques heures il a satisfait, non-seulement à la régularité du travail général sur toutes les parties du cristal, mais au rétablissement de la régularité dans la partie mutilée. "

Ces faits remarquables de rédintégration cristalline se rapprochent complétement de ceux que présentent les êtres vivants lorsqu'on leur fait une plaie plus ou moins profonde. Dans le cristal comme dans l'animal, la partie endommagée se cicatrise, reprend peu à peu sa forme primitive, et dans les deux cas le travail de reformation des tissus est en cet endroit bien plus actif que dans les conditions évolutives ordinaires. 
Les brèves considérations que nous venons d'exposer et que nous pourrions développer à l'infini nous semblent suffisantes pour montrer que la ligne profonde de démarcation que les vitalistes ont voulu établir entre les corps bruts au point de vue de leur durée, de leur évolution et de leur rédintégration formative, n'est pas fondée.

Quant à la lutte qu'ils ont supposée entre les forces ou les propriétés physiques et les forces ou les propriétés vitales, elle est l'expression d'une erreur profonde.

La doctrine des propriétés vitales enseigne qu'on ne trouve dans les corps bruts qu'un seul ordre de propriétés, les propriétés physiques, et que dans les corps vivants on en rencontre deux espèces, les propriétés physiques et les propriétés. vitales, constamment en lutte, en antagonisme, et tendant à prédominer les unes sur les autres.

"Pendant la vie, dit Bichat, les propriétés physiques, enchaînées par les propriétés vitales, sont sans cesse retenues dans les phénomènes qu'elles tendraient à produire. ” 
Il résultera logiquement de cet antagonisme que plus les propriétés vitales auront d'empire et domineront dans un organisme vivant, plus les propriétés physico-chimiques y seront vaincues et atténuées, et que, réciproquement, les propriétés vitales s'y montreront d'autant plus affaiblies que les propriétés physiques acquerront plus de puissance.

C'est précisément la proposition contraire qui exprime la vérité, et cette vérité a été surabondamment démontrée par les travaux de Lavoisier et de ses successeurs.

La vie est au fond l'image d'une combustion, et la combustion n'est elle-même qu'une série de phénomènes chimiques, auxquels sont reliées d'une manière directe des manifestations caloriques lumineuses et vitales. Qu'on supprime de l'atmosphère l'oxygène, l'agent des combustions, aussitôt la flamme s'éteint, aussitôt la vie s'arrête. Si l'on vient à diminuer ou à augmenter la quantité du gaz comburant, les phénomènes vitaux aussi bien que les phénomènes chimiques de combustion seront exaltés ou atténués dans la même proportion. 
Ce n'est donc pas un antagonisme qu'il faut voir entre les phénomènes chimiques et les manifestations vitales; c'est au contraire un parallélisme parfait, une liaison harmonique et nécessaire.

Dans toute la série des êtres organisée, l'intensité des manifestations, vitales est dans un rapport direct avec l'activité des manifestations chimiques organiques. De tous côtés, les preuves se présentent d'elles-mêmes.

Quand l'hornme ou l'animal est saisi par le froid, les phénomènes chimiques de combustion organique s'abaissent d'abord; puis les mouvements se ralentissent, la sensibilité, l'intelligence, s'émoussent et disparaissent, l'engourdissement est complet. Au réveil de cetle léthargie, les fonctions vitales reprennent, mais toujours parallèlement ̀̀ la réapparition des phénomènes chimiques.

Quand la vie se suspend chez un infusoire desséché et qu'elle se rétablit sous l'influence de quelques gouttes d'eau, ce n'est pas que la dessiccation ait attaqué la vie ou les propriétés vitales, c'est parce que l'eau nécessaire à la 
réalisation des phénomènes physiques et chimiques fait défaut à l'organisme. Quand Spallanzani a ressuscité, en les humectant, des rotifères desséchés depuis trente ans, il a simplement fait reparaître dans leur corps les phénomènes physiques et chimiques qui s'y étaient arrêtés pendant trente années. L'eau n’a apporté rien autre chose, ni force ni principe.

Comment pourrions-nous comprendre un antagonisme, une opposition entre les propriétés des corps vivants et celles des corps bruts, puisque les éléments constituants de ces deux ordres de corps sont les mêmes? Butfon, voulant s'expliquer la différence des êtres organisés et des êtres inorganiques, avait été logique en supposant chez les premiers une substance organique élémentaire spéciale dont seraient dépourvus les seconds. La chimie a complétement renversé cette hypothèse en prouvant que tous les corps vivants sont exclusivement formés d'éléments minéraux empruntés au milieu cosmique. Le corps de l'homme, le plus complexe des corps vivants, est matériellement constitué par quatorze de ces éléments. On comprend bien que 
ces quatorze corps simples puissent, en s'unissant, en se combinant de toutes les manières, engendrer des combinaisons infinies et formel des composés doués de propriétés les plus variées; mais ce qu'on ne concevrait pas, c'est que ces propriétés fussent d'un autre ordrẹ ou d'une autre essence que ces combinaisons ellesmêmes.

En résumé, l'opposition, l'antagonisme, la lutte admise entre les phénomènes vitaux et les phénomènes physico-chimiques sur ]'école vitaliste, est une erreur dont les découvertes de la physique et de la chimie modernes ont fail amplement justice.

Il y a plus, la doctrine vitaliste ne repose pas seulement sur des hypothèses fausses, sur des faits erronés; elle est par sa nature contraire ì l'esprit scientifique. En voulant créer deux ordres de sciences, les unes pour les corps bruts, les autres pour les corps vivants, cette doctrine aboutit purement et simplement à nier la science elle-même. Bichat, nous le savons déjà, pose en principe que les lois des sciences physiques sont absolument opposées aux lois des sciences 
vitales. Dans les premières, tout serait fixc et invariable; dans les secondes, tout serait variable et inconstant. La divergence entre ces deux ordres de sciences doit les laisser étrangères les unes aux autres et les rendre incapables de se prêter aucun secours. C'est la conclusion à laquelle arrive nécessairement Bichat. "Comme les sciences physiques et chimiques, dit-il, ont été perfectionnées avant les physiologigues, on a cru éclaircir les unes en y associant les autres; on les a embrouillées. C'était inévitable, car appliquer les sciences physiques à la physiologie, c'est expliquer par les lois des corps inertes les phénomènes des corps vivants. Or voilà un principe faux; donc toutes lesconséquences doivent être marquées au même coin. »

Si maintenant nous demandons quels sont les caractères propres à cette science des êtres vivants, Bichat nous répond : "C'est une science dont les lois sont, comme les fonctions vitales elles-mêmes, susceptibles d'une foule de variétés, qui échappe à toute espèce de calcul, dans laquelle on ne peut rien prévoir ou prédire, 
dans laquelle nous n'avons que des approsimations le plus souvent incertaines. "

Ce sont là des hérésies scientifiques d'une énormité telle qu’on aurait de la peine à les comprendre, si l'on ne voyait comment la logique d'un système a dû fatalement y conduire. Reconnaître que les phénomènes vitaux ne sauraient être soumis à aucune loi précise, à aucune condition fixe et déterminée, et admettre que ces phénomènes ainsi définis constituent une science vitale qui elle-même a pour caractère d'être vague et incertaine, e'est abuser étrangement du mot science. Il semble qu'il n'y ait rien à répondre à de pareils raisonnements, parce qu'ils ne sont eux-mêmes que la négation et l'absence de tout esprit scientifique.

Cependant que de fois n'a-t-on pas reproduil des arguments analogues, combien de médecins ont professé que la physiologie et la médecine ne seraient jamais que des demi-sciences, des sciences conjecturales, parce qu'on ne pourrail jamais saisir le principe de la vie ou le génic secret des maladies!

Ces affirmations, qui viennent encore retentir 
à nos oreilles comme des échos lointains de doctrines surannées, ne sauraient plus nous arrèter. Descartes, Leibniz, Lavoisier, nous ont appris que la matière et ses lois ne different pas dans les corps vivants et dans les corps bluts; ils nous ont montré qu'il n'y a au monde qu’une seule mécanique, une seule physique, une seule chimie, communes à tous les êtres de la nature. Il n'y a donc pas deux ordres de sciences.

Toute science digne de ce nom est celle qui, connaissant les lois précises des phénomènes, les prédit sûrement et les maîtrise quand ils sont à sa portée. Tout ce qui reste en dehors de ce caractère n'est qu'empirisme ou ignorance, car il ne saurait y avoir des demi-sciences ni des sciences conjecturales. C'est une erreur profonde de croire que dans les corps vivants nous ayons à nous préoccuper de l'essence même et du principe de la vie. Nous ne pouvons remonter au principe de rien, et le physiologiste n'a pas plus affaire avec le principe de la vie que le chimiste avec le principe de l'affinité des corps. Les causes premières nous échap- 
pent partout, et partout également nous ne pouvons atteindre que les causes immédiates des phénomènes. Or ces causes immédiates, qui ne sont que les conditions mêmes des phénomènes, sont susceptibles d'un déterminisme aussi rigoureux dans les sciences des corps vivants que dans les sciences des corps bruts. Il n'y a aucune différence scientifique dans tous les phénomènes de la nature, si ce n'est la complexité ou la délicatesse des conditions de leur manifestation qui les rendent plus ou moins difficiles à distinguer et à préciser.

Tels sont les principes qui doivent nous diriger. Aussi conclurons-nous sans hésiter que li dualité établie par l'école vitaliste dans les sciences des corps bruts et des corps vivants est absolument contraire à la science elle-même. L'unité règne dans tout son domaine. Les sciences des corps vivants et celles des corps bruts onl pour base les mêmes principes et pour moyens d'études les mêmes méthodes d'investigation. 


\section{III}

Si les doctrines vitalistes ont succombé par l'erreur essentielle de leur principe de dualisme ou d'antagonisme entre la nature vivante et la nature inorganique, le problème subsiste toujours. Nous avons à répondre à cette question séculaire : qu'est-ce que la vic? ou encore à cette autre: qu'est-ce que la mort? car ces deux questions sont étroitement liées et ne sauraient être séparées l'une de l'autre.

L'être vivant est essentiellement caractérisé par la nutrition. L'édifice organique est le siége d'un perpétuel mouvement nutritif, mouvement intestin qui ne laisse de repos à aucune partie; chacune, sans cesse ni trêve, s'alimente dans le milieu qui l'entoure et y rejette ses déchets et ses produits. Cette rénovation moléculaire est insaisissable pour le regard direct; mais, 
comme nous voyons le début et la fin, l'entrée et la sortie des substances, nous en concevons les phases intermédiaires, et nous nous représentons un courant de matières qui traverse continuellement l'organisme et le renouvelle dans sa substance en le maintenant dans sa forme. Ce mouvement qu'on a appelé le tourbillon vital, le circulus matériel entre le monde organique et le monde inorganique, existe chez la plante aussi bien que chez l'animal, ne s'interrompt jamais et devient la condition et en même temps la cause immédiate de toutes les autres manifestations vitales. L'universalité d'un tel phénomène, la constance qu'il présente, sa nécessité, en font le caractère fondamental de l'être vivant, le signe plus général de la vie. On ne sera donc pas étonné que quelques physiologistes aient été tentés de le prendre pour définir la vie elle-même.

Toutefois ce phénomène n'est pas simple; il importe del'analyser, d'en penétrer plus profondément le mécanisme, afin de préciser l'idée que son examen superficiel peut nous donner de la vie. 
Le mouvement nutritif comprend deux opérations distinctes, mais connexes et inséparables : l'une par laquelle la matière inorganique est fixée ou incorporée aux tissus vivants comme partie intégrante, l'autre par laquelle elle s'en sépare et les abandonne. Ce double mouvement incessant n'est en définitive qu'une alternative perpétuelle de vie et de mort, c'est-à-dire de destruction et de renaissance des parties constituantes de l'organisme.

Les vitalistes n'ont point compris la nutrition. Les uns, imbus de l'idée que la vie a pour essence de résister à la mort, c'est-à-dire aux forces physiques et chimiques, devaient croire naturellement que l'être vivant, arrivé à son plein développement, n’avait plus qu'à se maintenir dans l'équilibre le plus stable possible en neutralisant l'influence destructive des agents extérieurs; les autres, comprenant mieux le phénomène et appréciant la perpétuelle mutation de l'organisme, ont refusé d'admettre que ce mouvement de rénovation moléculaire füt produit par les forces générales de la nature, et ils l'ont attribué à une force vitale. 
Ni les uns ni les autres n'ont vu que c'était précisément la destruction organique, opérée sous l'influence des forces physiques et chimiques générales, qui provoque le mouvement incessant d'échange et devient ainsi la cause de la réorganisation.

Ies actes de destruction organique ou de désorganisation se révèlent immédiatement à nous; les signes en sont évidents, ils éclatent au dehors et se répètent à chaque manifestation vitale. Les actes d'assimilation ou d'organisation au contraire restent tout intérieurs et n'ont presque point d'expression phénoménale; ils président à une synthèse organique qui rassemble d'une manière silencieuse et cachée les matériaux qui seront dépensés plus tard dans les manifestations bruyantes de la vie. C'est une vérité bien remarquable et bien essentielle à saisir que ces deux phases du circulus nutritif se traduisent si différemment, l'organisation restant latente et la désorganisation ayant pour signe sensible tous les phénomènes de la vie. Ici l'apparence nous trompe, comme presque toujours; ce que nous appelons phénomène de 
vie est au fond un phénomène de mort organique.

Les deux facteurs de la nutrition sont done l'assimilation et la désassimilation, autrement ditl'organisation et la désorganisation. La désassimilation accompagne toujours la manifestation vitale. Quand chez l'homme et chez l'animal un mouvement survient, une partie de la substance active du muscle se détruit et se brûle; quand la sensibilité et la volonté se manifestent, les nerfs s'usent, quand la pensée s'exerce, le cerveau se consume, etc. On pent ainsi dire que jamais la même matière ne sert deux fois à la vie. Lorsqu'un acte est accompli, la parcelle de matière vivante qui a servi à le produire n'est plus. Si le phénomène reparaît, c'est une matière nouvelle qui lui a prêté son concours. L'usure moléculaire est toujours proportionnée à l'intensité des manifestations vitales. L'altération matérielle est d'autant plus profonde ou considérable que la vie se montre plus active. La désassimilation rejette de la pro. fondeur de l'organisme des substances d'autant plus oxydées par la combustion vitale que le fonctionnement des organes a été plus énergi- 
que. Ces oxydations ou combustions engendrent la chaleur animale, donnent naissance à l'acide carbonique qui s'exhale par le poumon, et à différents produits qui s'éliminent par les autres émonctoires de l'économie. Le corps s'use, éprouve une consomption et une perte de poids qui traduisent et mesurent l'intensité de ses fonctions. Partout, en un mot, la destruction physico-chimique est unie à l'activité fonctionnelle, et nous pouvons regarder.comme un axiome physiologique la proposition suivante : toute manifestation d'un phénomène dans l'être vivant est nécessairement liée à une destruction organique.

Une telle loi, qui enchaîne le phénomène qui se produit à la matière qui se détruit, ou, pour mieux dire, à la substance qui se transforme, n'a rien qui soit spécial au monde vivant; la nature physique obéit à la même règle.

Un être vivant qui est dans la plénitude de son activité fonctionnelle ne nous manifeste done pas l'énergie plus grande d'une force vitale mystérieuse; il nous offre simplement dans son organisme la pleine activité des phénomènes 
chimiques de combustion et de destruction organique. Quand Cuvier nous dépeint la vie s'épanouissant dans le corps d'une jeune femme ${ }^{1}$, il a tort de croire avec les vitalistes que les forces ou les propriétés physiques et chimiques sont alors domptées ou maintenues par la force vitale. Au contraire, toutes les forces physiques sont déchaînées, l'organisme brûle et se consume plus vivement, et c'est pour cela même que la vie brille de tout son éclat.

Stahl a dit avec raison que les phénomènes physiques et chimiques détruisent le corps vivant et le conduisent à la mort; mais la vérité lui a échappé pour ne pas avoir vı que les phénomènes de destruction vitale sont eux-mêmes les instigateurs et les précurseurs de la rénoyation matérielle qui se dérobe à nos yeux dans l'intimité des tissus. En même temps en effet que les phénomènes de combustion se traduisent avec éclat par les manifestations vitales extérieures, le processus formatif s'opère dans le silence de la vie végétative. Il n'a d'autre expression que lui-même, c'est-à-dire qu'il ne 1. Voy. p. 165. 
se révèle que par l'organisation et la réparation de l'édifice vivant.

On a dès l'antiquité comparé la vie à un flambeau. Cette métaphore est devenue de nos jours, grâce à Lavoisier, une vérité. L'être qui vit est comme le flambeau qui brûle; le corps s'use, la matière du flambeau se détruit; l'un brille de la flamme physique, l'autre brille de la flamme vitale. Toutefois, pour que la comparaison fût rigoureuse, il faudrait concevoir un flambeau physique capable de durer, qui se renouvelât et se régénérât comme le flambeau vital. La combustion physique est un phénomène isolé, en quelque sorte accidentel, n'ayant dans la nature de liaisons harmoniques qu'avec luimême. La combustion vitale au contraire suppose une régénération corrélative, phénomène de la plus haute importance dont il nous res'e à tracer les caractères principaux.

Le mouvement de régénération ou de synthèse organique nous offre deux modes principaux. Tantôt la synthèse assimile la substance ambiante pour en faire des principes nutritifs, tantôt elle en forme direclement les éléments 
des tissus. C'est ainsi que nous voyons, à côté de la formation des produits immédiats de la synthèse chimıque, apparaître des phénomènes de mues ou de rénovations histologiques, tantôt continues, tantôt périodiques. Les phénomènes derégénération, de rédintégration, de réparation, qui se montrent chez l'individu adulte, sont de la même natureque lesphénomènes de génération et d'évolution par lesquels l'embryon constitue à l'origine ses organes et ses éléments anatomiques. L’être vivant est donc caractérisé à la fois par la génération et par la nutrition; il faut réunir et confondre ces deux ordres de phénomènes, et, au lieu d'en créer deux catégories distinctes, nous en faisons un acte unique dont l'essence et les mécanismes sont tout pareils. C'est dans cette pensée que l'on a pu dire avec raison que la nutrition n'était qu'une généralion continuée. Synthèse organique, génération, régénération, rédintégration et même cicatrisation sont des aspects du même phénomène, des manifestations variées d'un même agent, le germe. Le germe est l'agent d'organisation et de nutrition par excellence; il attire autour de lui la 
matière cosmique et l'organise pour constituer l'être nouveau. Toutefois le germe ne peut manifester sa puissance organisatrice qu'en opérant lui-même des combustions, des destructions organiques. C'est pourquoi il s'enferme dès son origine dans une cellule, la cellule de l'œuf, et s'y entoure de matériaux nutritifs élaborés qu'on appelle le vilellus.

La cellule-œuf, ainsi constituée par le germe et le vitellus, développe l'organisme nouveau en se segmentant et se divisant à l'infini en une quantité innombrable de cellules pourvues elles-mêmes d'un germe de nutrition. Ce germe cellulaire, qu'on appelle le noyau de la cellule, attire et élabore autour de lui les matériaux nutritifs spéciaux destinés aux combustions fonctionnelles de chacun des éléments de nos tissus ou de nos organes. Lorsque des phénomènes de rédintégration naturels ou accidentels surviennent, lorsqu'un nerf coupé par exemple se régénère et reprend ses fonctions, ce sont encore ces noyaux cellulaires qui, à l'instar du germe primordial dont ils dérivent, se divisent, se multiplient, pour reconstituer chez l'adulte les 
tissus nouveaux en répétant identiquement les procédés de la formation embryonnaire.

Tous les phénomènes si variés de régénéra. tion et de synthèse organiques ont pour caractère distinctif, nous l'avons déjà dit, d'être .en quelque sorte invisibles à l'extérieur. Au silence qui se fait dans un œuf en incubation on ne pourrait soupconner l'activité qui s'y déploie et l’importance des phénomènes qui s'y accomplissent; c'est l'être nouveau qui en sortant nous dévoilera par ses manifestations vitales les merveilles de ce travail lent et caché.

Il en est de même de toutes nos fonctions; chacune a pour ainsi dire son incubation organisatrice. Quand un acte vital se produit extérieurement, ses conditions s'étaient dès longtemps rassemblées dans cette élaboration silencieuse et profonde qui prépare les causes de tous les phénomènes. Il importe de ne pas perJre de vue ces deux phases du travail physiologique. Quand on veut modifier les actions vitales, c'est dans leur évolution cachée qu'il faut les atteindre; lorsque le phénomène éclate, il est trop tard. Ici, comme partout, rien n'arrive 
par un brusque hasard; les événements les plus soudains en apparence ont eu leurs causes latentes. L'objet de la science est précisément de découvrir ces causes élémențaires, afin de pouvoir les modifier et maîtriser ainsi l'apparition ultérieure des phénomènes.

En résumé, nous distinguerons dans le corps vivant deux grands groupes de phénomènes inverses : les phénomènes fonctionnels ou de dépense vitale, les phénomènes organiques ou de concentration vitale. La vie se maintient par deux ordres d'actes entièrement opposés dans leur nature : la combustion désassimilatrice, qui use la matière vivante dans les organes en fonction, la synthèse assimilatrice, qui régénère les tissus dans les organes en repos. Les agents de ces deux genres de phénomènes ne sont pas moins différents. La combustion vitale emprunte à l'extérieur l'agent général des combustiors, l'oxygène, et à son défaut les ferments dont l'action désassimilatrice peut intervenir dans les profondeurs de l'organisme où l'air ne pénètre pas. La synthèse organisatrice au contraire possède un agent spécial, le germe pro- 
prement dit, ou les noyaux de cellules, germes secondaires qui en sont des émanations et qui se trouvent répandus dans toutes les parties élémentaires du corps vivant. Les conditions de la désassimilation fonctionnelle et celles de l'assimilation organique sont également séparées. Les mêmes agents de combustion qui usent l'édifice organique pendant la vie continuent à le détruire après la mort lorsque les phénomènes de régénération se sont éteints dans l'organisme. Il en résulte que tous les phénomènes fonctionnels accompagnés de combustion, de fermentation ou de dissociation organique, peuvent s'accomplir aussi bien au dehors qu'au dedans des corps vivants.

Grâce à cette circonstance, le physiologiste peut analyser les mécanismes vitaux à l'aide de l'expérimentation. Dans un organisme mutilé, il entretient artificiellement la respiration, la circulation, la digestion, etc., et il étudie les propriétés des tissus vivants séparés du corps. Dans ces parties disloquées, le muscle se contracte, la glande sécrète, le nerf conduit les excitations absolument comme pendant la vie; 
DÉFINITION DE LA VIE.

toutefois, si les tissus isolés de l'ensemble de leurs conditions organiques peuvent s'user et fonctionner encore, ils ne peuvent plus se régénérer : c'est pourquoi leur mort définitive devient alors inévitable. Les phénomènes de rénovation organique, contrairement aux phénomènes de combustion fonctionnelle, ne peurent se manifester que dans le corps vivant, et chacun dans un lieu spécial; aucun artifice n'a pu jusqu'à présent suppléer à ces conditions essentielles de l'activité des germes, d'êtrè en leur place dans l'édifice du corps vivant.

Si on se fondait sur les différences profondes que nous venons d'indiquer pour assigner dans l'économie un rôle vital indépendant à la combustion et à la régénération organique, on se tromperait grandement, car les deux ordres de phénomènes sont tellement solidaires dans l'acte de la nutrition, qu'ils ne sont pour ainsi dire distincts que dans l'esprit; dans la nature, ils sont inséparables. Tout être vivant, animal ou végétal, ne peut manifester ses fonctions que par l'exercice simultané de la combustion vitale et de la synthèse organique. C'est sur ce ler- 
rain que devront se réunir et se concilier les écoles chimiques et anatomiques, car la solution du problème physiologique de la vie exige leur double concours'.

\section{IV}

Nous avons poursuivi le phénomène caractéristique de la vie, la nutrition, jusque dans ses manifestations intimes; royons quelle conclusion cette étude peut nous fournir relatirement à la solution du problème tant de fois essayé de la définition de la vie.

Si nous voulions exprimer que toutes les fonctions vitales sont la conséquence nécessaire d'une combustion organique, nous répéterions ce que nous avons déjà énoncé : la vie, c’est la mort, la destruction des tissus, ou bien nous

1. Voyez Claude Bernard, Lecons sur les phénomènes de la vie communs aux animanx et aux végélaux. Paris, 1878. 
dirions avec Buffon : la vie est un minotaure, elle dévore l'organisme.

Si au contraire nous voulions insister sur cette seconde face du phénomène de la nutrition, que la vie ne se maintient quà la condition d'une constante régénération des tissus, nous regarderions la vie comme une création exécutée au moyen d'un acte plastique et régénérateur opposé aux manifestations vitales.

Enfin, si nous voulions comprendre les deux faces du phénomène, l'organisation et la désorganisation, nous nous rapprocherions de la définition de la vie donnée par de Blainville: « La vie est un double mouvenent interne de décomposition à la fois général et continu."

Plus récemment Herbert-Spencer a proposé la définition suivante: " La vie est la combinaison définie de changements hétérogènes à la fois simultanés et successifs; " sous cette définition abstraite, le philosophe anglais veut surtout indiquer l'idée d'évolution et de succession qu'on observe dans les phénomènes vit:ux.

De telles définitions, tout incomplètes qu'elles 
soient, auraient au moins le mérite d'exprimer un aspect de la vie : elles ne seraient point purement verbales, comme celle de l'Encyclopédic: " la vie est le contraire de la mort, » ou encore celle de P. A. Béclard : “ la vie est l'organisation en action, » celle de Dugès : “ la vie est l'activité spéciale des êtres organisés, » ce qui revient à dire : la vie, c'est la vie.

Kant a défini la vie : " un principe intérieur d'action. »

Cette définition, qui rappelle l'idée d'Hippocrate $^{1}$, a été adoptée par Tiedemann et par d'autres physiologistes. Il n'y a en réalité pas plus de principe intérieur d'activité dans la matière vivante que dans la matière brute. Les phénomènes qui se passent dans les minéraux sont certainement sous la dépendance des conditions atmosphériques extérieures; mais il en est de même de l'activité des plantes et des animaux à sang froid. Si l'homme et les animaux à sang chaud paraissent libres et indépendants dans leurs manifestations vitales, cela tient à ce que

1. Hippocrate, Euvres complètes, trad. Littré. Paris, 1840. 
leur corps présente un mécanisme plus parfait qui leur permet de produire de la chaleur en quantité telle qu'il n'a pas besoin de l'emprunter nécessairement au milieu ambiant. En un mot, la spontanéité de lia matière vivante n'est qu'une fausse apparence. Il y a constamment des principes extérieurs, des stimulants étrangers qui viennent provoquer la manifestation des propriétés d'une matière toujours également inerte par elle-même.

Nous bornerons ici ces citations, que nous pourrions multiplier à l'infini sans trouver une seule définition complétement satisfaisante de la vie. Pourquoi en est-il ainsi ? C'est qu'à propos de la vie il faut distinguer le mot de la chose elle-même. Pascal, qui a si bien connu toutes les faiblesses et toutes les illusions de l'esprit humain, fait remarquer qu'en réalité les vraies définitions ne sont que des eréations de notre esprit, c'est-à-dire des définitions de noms ou des conventions pour abréger le discours; mais il reconnaît des mots primitifs que l'on comprend sans qu’il soit besoin de les délinir. 
Or le mol vie est dans ce cas. Toui le monde s'entend quand on parle de la vie et de la mort. Il serait d'ailleurs impossible de séparer ces deux termes ou ces deux idées corrélatives, car ce qui vit, c'est ce qui mourra, ce qui est mort, c'est ce qui a vécu. Quand il s'agit d'un phénomène de la vie comme de tout phénomène de la nature, la première condition est de le connaître; la définition ne peut être donnée qu'à posteriori, comme conclusion résumée d'une étude préalable; mais ce n'est plus là, à proprement parler, une définition; c'est une vue, une conception. Il s'agira donc pour nous de savoir quelle conception nous devons nous former des phénomènes de la vie aujourd'hui dans l'état actuel de nos connaissances physiologiques.

Cette conception a varié nécessairement avec les époques et suivant les progrès de la science.

Au commencement de ce siècle, un physiologiste francais, Le Gallois, publiait encore un rolume d'expériences : sur le Principe de la vie el sur le siége de ce principe. On ne cherche plus maintenant le siége de la vie; on sait qu'elle réside partout dans toutes les molécules de la 
matière organisée. Les propriétés vitales ne sont en réalité que dans les cellules vivantes, tout le reste n'est qu'arrangement et mécanisme. Les manifestations si variées de la vie sont des expressions mille et mille fois combinées et diversifiées de propriétés organiques élémentaires fixes et invariables. Il importe donc moins de connaître l’immense variété des manifestations vitales que la nature semble ne pouvoir jamais épuiser que de déterminer rigoureusement les propriétés de tissus qui leur donnent naissance. C'est pourquoi aujourd'hui tous les efforts de la science sont dirigés vers l'étude histologique de ces infiniment petits qui recèlent le véritable secret de la vie.

Aussi loin que nous descendions aujourd'hui dans l'intimité des phénomènes propres aux ètres vivants, la question qui se présente à nous est toujours la même. C'est la question qui a été posée dès l'antiquité au début même de la science: la vie est-elle due à une puissance, à une force particulière, ou n'est-elle qu'une modalité des forces générales de la nature? en d'autres termes, existe-t-il dans les êtres rivants une force 
spéciale qui soit distincte des forces physiques, chimiques ou mécaniques?

Les vitalistes se sont toujours retranchés dans l'impossibilité d'expliquer physiquement ou mécaniquement tous les phénomènes de la vie; leurs adversaires ont toujours répondu en réduisant un plus grand nombre de manifestations vitales à des explications physico-chimiques bien démontrées. Il faut avouer que ces derniers ont constamment gagné du terrain et qu'à notre époque surtout ils en gagnent chaque jour de plus en plus. Arriveront-ils ainsi à tout ramener à leurs théories et ne restera-t-il pas malgré leurs efforts un quid proprium de la vie qui sera irréductible? C'est le point qu'il s'agit d'examiner. En analysant avec soin tous les phénomènes vitaux dont l'explication appartient aux forces physiques et chimiques, nous refoulerons le vitalisme dans un domaine plus circonscrit et dès lors plus facile à déterminer.

Des deux ordres de phénomènes nutritifs qui constituent essentiellement la vie et qui sont l'origine de toutes ses manifestations saus exception, il en est un, celui de la destruction, 
de la désassimilation organique, qui rentre complétement dès maintenant dans les actions chi miques; ces décompositions dans les ètres vivants n'onl rien de plus ou moins mystérieux que celles qui nous sont offertes par les corps inorganiques.

Quant aux phénomènes de genèse organisatrice et de génération nutritive, ils paraissent au premier abord d'une nature vitale tout à fait spéciale, irréductibles aux actions chimiques générales; mais ce n'est encore là qu'une apparence, et pour bien s'en rendre compte il faut considérer ces phénomènes sous le double aspect qu'ils présentent d'une synthèse chimique ordinaire et d'une évolution organique qui s'accomplit. En effet, la genèse vitale comprend des phénomènes de synthèse chimique arrangés, développés suivant un ordre particulier qui constitue leur évolution. Il importe de séparer les phénomènes chimiques en eux-mêmes de leur évolution, car ce sont deux choses tout à fait distinctes.

En tant qu'actions synthétiques, il est évident que ces phénomènes ne relèrent que des forces CLAUDE BERNARD. 
chimiques générales; en les examinant successivement un à un, on le démontre clairement. Les matières calcaires qu'on rencontre dans les coquilles des mollusques, dans les œufs des oiseaux, dans les os des mammifères, sont bien certainement formées selon les lois de la chimie ordinaire pendant l'évolution de l'embryon. Les matières grasses et huileuses sont dans le même cas, et déjà la chimie est parvenue ì reproduire artificiellement dans les laboratoires un grand nombre de principes immédiats et d'huiles essentielles, qui sont naturellement l'apanage du règne animal ou végétal. De même les matières amylacées, qui se développent dans les animaux et qui se produisent par l'union du carbone et de l'eau sous l'influence du soleil dans les fouilles vertes des plantes, sont bien des phénomènes chimiques les mieux caractérisés. Si pour les matières azotées ou albuminoïdes les procédés de synthèse sont beaucoup plus obscurs, cela tient à ce que la chimie organique est encore trop peu avancée; mais il est bien certain néanmoins que cęs substances se forment par les procédés chimiques dans les 
organismes des êtres vivants. A la vérité, on peut dire que les agents des synthèses organiques, les germes et les cellules, constituent des agents tout à fait exceptionnels.

On pourrait dire de même pour les phénomènes de désorganisation que les ferments sont aussi des agents particuliers aux êtres vivants. Je pense, quant à moi, que c'est là une loi générale et que les phénomènes chimiques dans l'organisme sont exécutés par des agents ou des procédés spéciaux; mais cela ne change rien à la nature purement chimique des phénomènes qui s'accomplissent et des produits qui en sont la conséquence.

Après avoir examiné la synthèse chimique, arrivons à l'évolution organique.

Les agents des phénomènes chimiques dans les corps vivants ne se bornent pas à produire des synthèses chimiques de matières extrêmement variées, mais ils les organisent et les approprient à l'édification morphologique de l'être nouveau Parmi ces agents de la chimie vivante, le plus puissant et le plus merveilleux est sans contredit l'œuf, la cellule pri- 
mordiale qui contient le germe, principe organisateur de tout le corps. Nous n'assistons pas à la création de l'œuf ex nihilo, il vient des parents, et l'origine de sa virtualité évolutive nous est cachée; mais chaque jour la science remonte plus haut vers ce mystère. C'est par le germe, et en vertu de cette sorte de puissance évolutive qu'il possède, que s'établissent la perpétuité des espèces et la descendance des êtres; c'est par lui que nous comprenons les rapports nécessaires qui existent entre les phénomènes de la nutrition et ceux du développement. Il nous explique la durée limitée de l'être vivant, car la mort doit arriver quand la nutrition s'arrête, non parce que les aliments font défaut, mais parce que l'enchaînement évolutif de l'être est parvenu à son terme, et que l'impulsion cellulaire organisatrice a épuisé sa vertu.

Le germe préside encore à l'organisation de l'être en formant, à l'aide des matières ambiantes, la substance vivante, et en lui donnant les caractères d'instabilité chimique qui deviennent la cause des mouvements vitaux incessants qui 
se passent en elle. Les cellules, germes secondaires, président de la même façon à l'organisa? tion cellulaire nutritive. Il est bien évident que ce sont des actions purement chimiques; mais il est non moins clair que ces actions chimiques en vertu desquelles l'organisme s'accroît et s'édifie s'enchaînent et se succèdent en vue de ce résultat qui est l'organisation et l'accroissement de l'individu animal ou végétal. Il y a comme un dessin vital qui trace le plan de chaque être et de chaque organe, en sorte que, si, considéré isolément, chaque phénomène de l'organisme est tributaire des forces générales de la nature, pris dans leur succession et dans leur ensemble, ils paraissent révéler un lien spécial; ils semblent dirigés par quelque condition invisible dans la route qu'ils suivent, dans l'ordre qui les enchaîne. Ainsi les actions chimiques synthétiques de l'organisation et de la nutrition se manifestent comme si elles étaient dominées par une force impulsive gouvernant la matière, faisant une chimie appropriée à un but et mettant en présence les réactifs aveugles des laboratoires, à la manière du chimiste lui- 
mème. Cette puissance d'évolution immanente à l'ovule qui doit reproduire un être vivant embrasse à la fois, ainsi que nous ln savons déjà, les phénomènes de génération et de nutrition; les uns et les autres ont donc un caractère évolutif qui en est le fond et l'essence.

C'est cette puissance ou propriété évolutive que nous nous bornons à énoncer ici qui seule constituerait le quid proprium de la vie, car il est clair que cette propriété évolutive de l'œuf, qui produira un mammıfère, un oiseau on un poisson, n'est ni de la physique, ni de la chimie. Les conceptions vitalistes ne peuvent plıs aujourd'hui planer sur l'ensemble de la physiologie. La force évolutive de l'œuf et des cellules est donc le dernier rempart du vitalisme; mais en s'y réfugiant, il est aisé de voir que le vitalisme se transforme en une conception métaphysique et brise le dernier lien qui le rattache au monde physique, à la science physiologique.

En disant que la vie est l'idée directrice ou la force évolutive de l'être, nous exprimons simplement l'idée d'une unité dans la succession de 
tous les changements morphologiques et chimiques accomplis par le germe depuis l'origine jusqu'à la fin de la vie. Notre esprit saisit cette unité comme une conception qui s'impose à lui, et il l'explıque par une force; mais l'erreur serait de croire que cette force métaphysique est active à la facon d’une force physique. Cette conception ne sort pas du domaine intellectuel pour venir réagir sur les phénomènes pour l'explication desquels l'esprit l'a créée; quoique émanée du monde physique, elle n'a pas d'efiet rétroactif sur lui.

En un mot, la force métaphysique évolutive par laquelle nous pouvons'caractériser la vie est inutile à la science, parce qu'étant en dehors des forces physiques elle ne peut exercer aucune influence sur elles. Il faut donc ici séparer le monde métaphysique du monde physique phénoménal qui lui sert de base, mais qui n'a rien à lui emprunter. Leibniz a exprimé cette délimitation dans des paroles que nous rappelions au dẻbut de cette étude; la science la consacre . aujourd'hui.

En résumé, si nous pouvons définir la vie à 
l'aide d'une conception métaphysique spéciale, il n'en reste pas moins vrai que les forces mécaniques, physiques et chimiques, sont seules les agents effectifs de l'organisme vivant, et que le physiologiste ne peut avoir à tenir compte que de leur action.

Nous dirons avec Descartes : on pense mélaphysiquement, mais on vil et on agit physiquement. 


\section{LA GHALEUR ANIMALE}

J'ai cherché à contrôler les expériences multiples qui ont été faites sur ce point de physiologie et je vais exposer le résultat de mes recherches ${ }^{1}$.

Il y a dans cette question de la chaleur animale deux points. Je ne m'étendrai que sur un seul, celui de la topographie culorifique.

A tour de rôle, on a placé le siége de la chialeur animale dans le poumon, dans les capillaires, dans le tissu musculaire, etc.

A mon avis, il n'existe pas de foyer unique: la chaleur se fait partout, mais il y a des points

1. Voyez Cl. Bernard, Leçons sur la chaleur animale, sur les effets de la chaleur et sur la fièvre. Paris, 1876. 
oì elle est plus élevée, tout en étant réglée par les lois définies.

Le premier point que l'on a discuté est celui de savoir si le sang artériel est plus chaud que le sang veineux, si le sang du cœur gauche est plus chaud que le sang du coeur droit. La théorie de Lavoisier était venue donner un solide appui à l'opinion qui défendait la tempé* rature plus élevée du sang artériel. Mes recherches combattent absolument cette façon de voir, et les erreurs d'interprétation tiennent à des vices d'expérimentation.

Les méthodes et les procédés ont varié beaucoup. Voici celle que j'ai adoptée.

Je prends deux aiguilles galvano-électriques, construites d'une facon spéciale et introduites dans une sonde de gomme analogue à la vulgaire sonde chirurgicale. Cette sonde est destinée à empêcher le contact du liquide sanguin avec l'aiguille. Des olsservations comparées et répétées permettent d'affirmer que cette enveloppe protectrice ne gêne en rien l'exactitude de cet appareil thermométrique. Il se borne du reste à mesurer les 1/50 de degré. 
Je prends un chien, auquel je découvre les artères et veines crurales, et j'introduis dans les deux vaisseaux ma sonde aiguillée. La sonde restant à l'entrée, j'ai constamınent observé l: résultat suivant : la température du sang artériel est plus élevée que celle du sang veineus. Aussi loin qu'on pousse la sonde dans l'artère (jusqu'à la crosse de l'aorte), la température resle invariable.

$\mathrm{Si}$, au contraire, on fait remonter la sonde dans le conduit veineux, la température varie: à l'entrée de la veine, elle est au-dessous de celle du sang artériel; elle augmente progressivement, pour être égale au niveau des veines rénales et atteindre son maximum au niveau du diaphragme, au point où les veines sushcipatiques s'abouchent dans la veine cave; audessus, elle diminue un peu, quoique restant toujours au-dessus de celle du sang artériel.

Cette différence entreles deux températures est fondamentale, et si l'on ne l'observe pas dans les vaisseaux des membres, c'est que le sang subit à la périphérie des déperditions multiples qui lui font perdre sa puissance calorique. 
Au sujet de ces expériences, j’ai observé un lait intéressant.

J'avais gardé un chien sur lequel j'avais pratiqué ces recherches; le lendemain, le chien était en proie à une fièvre des plus intenses. J'eus l'idée de rechercher si le rapport était le même dans cet état : il l'était en effet, mais avec des différences beaucoup plus prononcées.

Je lui fis prendre alors une forte dose d'opium : la température ne fut pas abaissée. Cependant à l'état normal l'opium amène un abaissement considérable de la chaleur.

Heidenhain avait observé qu'une excitation nerveuse amène un abaissement de tempéraŁure; si l'animal étaiı fébricitant, la même excilation ne produisait aucune modification. Ces faits peuvent être rapprochés de mes expériences avec l'opium.

On peut tirer de ces recherches l'idée clinique suivante : c'est que la fièvre est un phénomène purement nerveux provenant des modifications, des troubles qui se passent du côté du système nerveux. Appuyé sur des investigations nom- 
breuses, je crois qu'il existe des nerf's vasomoteurs de deux ordres, dilatateurs et constricteurs. La fièvrẻ n'est que la résultante de modifications profondes du côté de ce système, résultante qui a pour effet principal l'élévation de la température.

Association fri _çaise pour l'avancement des Sciences. Session de Nantes, 20 août 1875. 


\section{LA SENSIBILITÉ}

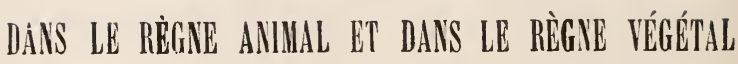

Mon but est de montrer que les plantes possèdent comme les animaux, au degré ou à la forme près, la sensibilité, cet attribut essentiel de la vie.

Réunissant la sensibilité consciente, la sensibilité inconsciente, l’irritabilıté, je crois établir, en mappuyant de mes recherches nouvelles, que ce sont là trois expressions graduées d'une seule et unique propriété, la sensibilité, la possession de cette faculté commune démontrant l'unité fonctionnelle des êtres vivants, depuis la plante la plus dégradée jusqu’à l'animal le plus élevé en organisation ${ }^{1}$.

Les philosophes ne connaissent et n'admet-

1. Voy. Cl. Bernard, Leçons sur les phénomènes de la vie communs aux animaux ét aux végétaux. Paris, 1878. 
tent en général que la sensibilité consciente, celle qu'atteste le moi. C'est pour eux la modification psychique, plaisir, douleur, déterminée par les modifications externes. Une telle définition ne s'applique guère qu'à l'homme seul, puisqu'elle fait intervenir la conscience : le phénomène qu'elle caractérise est sans analogue, sans pair, on pourrait dire sans signification, dès que l'on sort du sujet pensant.

Les physiologistes se placent nécessairement à un autre point de vue. Il ne leur suffit pas de définir, ils doivent étudier le phénomène objectivement, sous toutes les formes qu'il revêt. Ils observent qu'au moment où un agent modificateur vient agir sur l'homme, il ne provoque point seulement le plaisir ou la douleur, il n'affecte pas seulement l'âme : il affecte le corps, il détermine d'autres réactions que les réactions psychiques, et ces réactions automatiques, loin d'être la partie accessoire du phénomène, en sont au contraire l'élément essentiel, persistant, survivant aux autres réactions choz l'homme même, seules saisissables chez les autres animaux. 
Le nom de sensibilité désigne donc, aux yeux du physiologiste, l'ensemble des modifications de toute nature, déterminées dans l'être vivant par les stimulants, ou mieux l'aptitude à répondre par ces modifications à la provocation des stimulants.

Quand l'œil, l'oreille ou les papilles de la peau subissent l'action des agents physiques, vibration lumineuse, vibration sonore, vibration calorifique ou contact, la modification physiologique qu'ils subissent, le physiologiste doit l'appeler sensibilité. La sensation n'est qu'un élément de ce complexus qui peut faire défaut, les autres subsistant.

Le musicien qui déchiffre machinalement un morceau de musique, emporté dans une distraction qui voile sa conscience, recoit l'impression lumineuse et réagit de la même manière, au phénomène psychique près, que lorsque son attention est éveillée.

Les choses se passent de même quand les aliments pénètrent dans l'estomac et viennent irriter la membrane muqueuse qui le tapisse : l'observateur dont le regard pourrait pénétrer 
jusque-là verrait, comme l'a vu le docteur W. Beaumont, sur un Canadien dont l'estomac était resté ouvert à la suite d'une blessure d'arme à feu, il verrait, disons-nous, sous l'action des aliments ou de toute substance introduite dans la cavité, la muqueuse rougir, se tuméfier et se couvrir d'une sécrétion particulière. Voilà une réaction bien remarquable et bien évidente dont le moi n'a pas connaissance.

Il en est de même pour le cœur qui réagit ì ses stimulants, sans que nous en soyons directement prévenus ${ }^{1}$.

Il en est encore ainsi de tous les mouvements organiques soustraits à notre connaissance et ì notre volonté.

Dans tous ces exemples, la nature des réactions vitales est variable, la propriété de réagir est commune. En dehors du système nerveux, la propriété de réagir, identique au fond, appar= tient à tous les tissus, à tous les éléments anatomiques de l'organisme. Les physiologistes, depuis Haller et Glisson, ont désigné par le

1. Voy. le Cour, p. 316. 
nom d'irritabilité ce privilége commun des tissus animaux. Toutefois, bien des idées confuses ont obscurci la notion de l'irritabilité, jusqu'au jour où Bichat la présenta sous un aspect nouveau.

Bichat distinguait trois expressions de la sensibilité :

$1^{\circ}$ La sensibilité consciente, qui préside à la vie de relation ou aux mouvements extérieurs;

$2^{\circ}$ La sensibilité inconsciente, qui se traduit par les mouvements internes;

$3^{0}$ La sensibilité insensible, c'est-à-dire insaisissable à l'œil parce qu'elle se manifeste autrement que par des mouvements, par exemple par des actions nutritives ou trophiques.

Pour moi, me placant au point de vue de la conception des organismes vivants, telle que je l'ai exposée ailleurs, je considère la sensibilité comme une des propriétés fondamentales de tous les éléments organiques, de toute cellule vivante. Quand la sensibilité se traduit dans un élément isolé, nous ne lui connaissons pas d'appareils nerveux distincts; quand elle est l'expression plus complexe de la sensibilité de 
divers éléments, tissus ou organes, qu'elle harmonise, elle emprunte des appareils nerveux qui se montrent eux-mêmes plus ou moins compliqués suivant la nature des phénomènes qu'ils expriment. Enfin, quand la sensibilité nous apparaît comme une réaction de l'organisme entier, elle représente le consensus vital le plus élevé, et c'est dans ce cas seulement qu'elle devient consciente dans l'homme et dans les organismes supérieurs.

A considérer les choses objectivement, on trouve donc tous les degrés et toutes les formes depuis la sensibilité consciente jusqu'à l'obscure réaction du tissu, le fait conscience qui vient compliquer le complexus sensibilité qui dépend de cette circonstance que l'irritation a porté sur une partie en relation avec le cerveau, siége du sensorium commun. En un mot, la sensibilité est la propriété de réagir d'une façon appréciable mais plus ou moins visible, sous l'influence d'une sollicitation extérieure.

Prise dans ce sens général, la sensibilité se confond arec l'irritabilité. La sensibilité proprement dite et l'irritabilité particulière du tissu 
ou de l'élément nerveux, comme l'irritabilitė d'un tissu quelconque, peut être appelée la sensibilité particulière de cet élément ou de ce tissu Toutes ces formes de la sensibilité se confondent et sont identiques. La communauté d'essence et l'identité fondamentale est démontrée par la communauté des anesthésiques de l'identité des circonstances qui la font disparaître ou l'abolissent.

C'est ainsi que la sensibilité nous apparaîtra maintenant comme la propriété la plus caractéristique et la plus générale de la vie. Tout ce qui vit sent et peut être anesthésié; tout ce qui ne sent pas ne vit pas et ne peut être anesthésié, dirons-nous ${ }^{1}$.

La sensibilité ou irritabilité considérée ainsi comme l'attribut universel de la vie doit appartenir dès lors tout autant aux végétaux qu'aux animaux, sans quoi notre formule serait inexacte et notre généralisation illégitime.

Et en effet, les végétaux possèdent la sensi-

1. Voy. Glaude Bernard, Leçons sur les anesthésiques et sur l'asphyxie. Paris, 1875. 
bilité au même titre et aux mêmes conditions que tous les êtres animés. La diagnose exclusive de Linné: vegetabilia crescunt et vivunt; animalia crescunt, vivunt et sentiunt, n'est pas exacte en ce qu'elle s'en tient aux apparences et comme à l'écorce des choses.

On sait depuis longtemps que certaines plantes réagissent quand on les touche: ainsi la sensitive ferme ses feuilles au contact des mains qui veulent les saisir.

Mais ces phénomènes étaient regardés comme tout à fait exceptionnels, et leur réalité ne pas sait même pas pour absolument démontrée.

La généralisation que j'ai présentée a pris un caractère tout nouveau parce qu'on connaît maintenant un véritable réactif de la vie et de la sensibilité qui permet d'en reconnaître partout avec certitude l'existence.

Ce réactif c'est l'agent anesthésique, soit l'éther, soit le chloroforme.

Tout le monde connaît l'emploi de l'éther ou du chloroforme pour suspendre momentanément la sensibilité consciente, et chacun sait que le but poursuivi est précisément la suppres- 
sion de la douleur qui accompagne cette sensibilité consciente pendant les opérations chirurgicales.

On fait respirer les vapeurs d'éther ou de chloroforme qui arrivent dans les poumons, à travers les parois des vésicules pulmonaires, elles pénètrent alors dans le sang qui les conduit au contact des éléments nerveux de l'encéphale; c'est alors que le moi s'endort et avec lui la sensibilité consciente.

On ne pousse pas l'action plus loin parce qu'elle n'aurait plus aucune utilité chez le malade qu'on opère. Mais si nous éthérisons des animaux, comme des grenouilles, en continuant indéfiniment l'introduction des vapeurs d'éther, nous voyons successivement s'éteindre, après la sensibilité consciente, toutes les manifestations de la sensibilité inconsciente dans l'intestin et les glandes, et nous finissons par arrêter l'irritabilité musculaire et les agitations si vivaces des cils vibratils implantés en trèsgrand nombre comme les poils d'une brosse dans certaines membranes muqueuses, par exemple celle qui tapisse les voies respiratoires. 
L'éther ou le chloroforme n'exercent donc pas seulement leur action sur les organes nerveux: quand on laisse leurs effets se compléter, ils agissent de la même manière en supprimant la propriété de réagir dans tous les tissus, quelle qu'en soit la nature et la forme. Il n'y a d'autre différence que celle même qui sépare l'intensité de ces diverses réactions ou le degré de leur rapidité.

Ce sont aussi des différences du même genre qui séparent les plantes des animaux, c'est-ìdire les simples différences de degré, et l'éther, comme le chloroforme, exerce sur elles une action identique à celle qu'on vient de constater chez les animaux. Soumettez aux vapeurs d'éther ou de chloroforme les feuilles d'une sensitive, et vous pourrez toucher ces feuilles sans qu'elles réagissent comme d'ordinaire: elles ne sentent plus le contact des mains (fig. 1).

Ce premier fait déjà constaté me conduisit ì croire qu'on pouvait le reproduire sur les autres organes et à propos des autres fonctions des plantes; comme on avait étendu chez les animaus 
l'anesthésie du cerveau, qui est le siége de la sensibilité consciente, à tous les autres tissus où résident la sensibilité inconsciente et l'irritabilité.

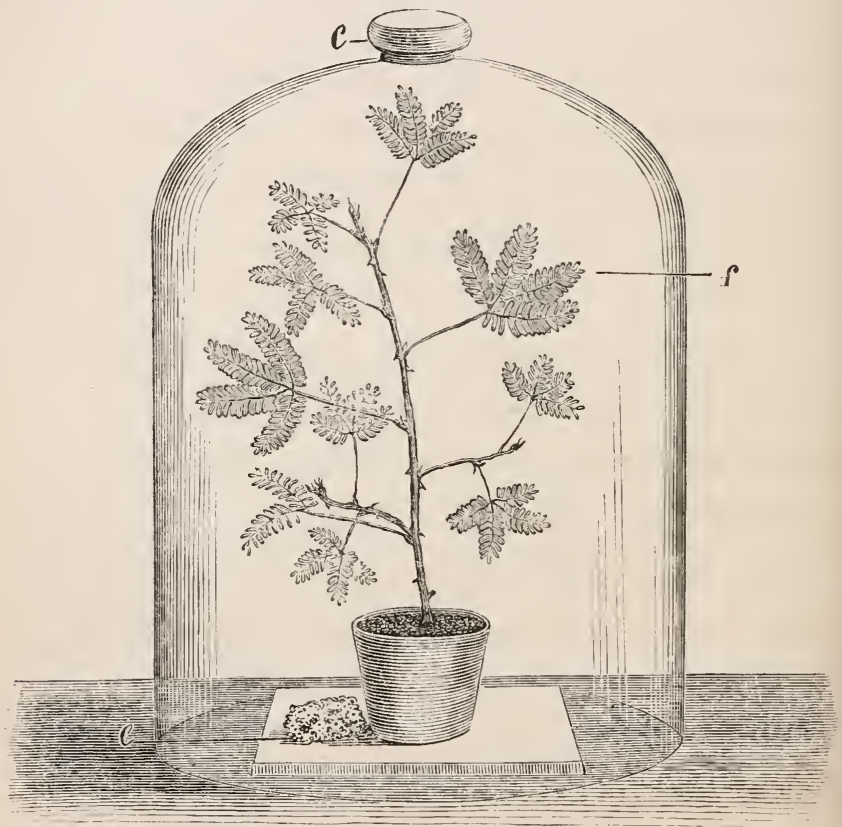

Fig. 1. Sensitive (Mimosa pudica) placée dans une atmosphère éthẻrée. $-e$, éponge imbibée d'éther ${ }^{1}$.

Prenez une graine à germination très-rapide,

1. Les feuilles de la plante sont étalées, sont devenues insensibles, et ne se ferment plus quand on vient à les toucher. 


\section{LA SENSIBILITÉ.}

comme celle de certains cressons, et placez-la sur une éponge imbibée d'eau: le lendemain elle aura déjà germé et poussé une tigelle et une radicelle. Répétez maintenant l'expérience en placant l'éponge sous une cloche dans laquelle parviennent des vapeurs d'éther, la graine y restera inerte, quoiqu'elle ait à sa disposition de l'oxygène, de l'eau, de la lumière, de la chaleur; elle ne sent plus les excitants qui l'entourent.

Ne croyez pas cependant qu'elle soit morte ou atteinte dans quelque organe essentiel: elle dort simplement, comme vous pouvez vous en convaincre aisément.

Levez la cloche, les vapeurs d'éther se dissiperont, la graine sortira de son sommeil, et dès le lendemain, elle entrera en germination '.

On reproduira la même observation sur un œuf de poule qui ne serait jamais couvé efficacement dans une atmosphère éthérée.

Passons maintenant à un autre phénomène de la vie des plantes, celui qu'on appelle encore

1. Voy. Lerons sur les phénomènes de la vie rommuns aux animanx et anx végétrux. Paris, 1878 p. 73. 
improprement leur respiration, je veux parler de la fonction au moyen de laquelle la plante absorbe de l'acide carbonique et rejette dans l'air de l'oxygène.

Tout le monde sait que ce phénomène siégeant dans les parties vertes exige l'action de la lumière; il se produit ailleurs tout aussi bien, si ce n'est mieux, dans les feuilles des plantes aquatiques plongées sous l'eau, que dans les feuilles des plantes aériennes.

Eh bien, prenez une plante aquatique et pla. cez-la dans un bocal que vous aurez rempli d'eau tenant en dissolution de l'éther ou du chloroforme. C'est une expérience que chacun peut répéter aisément, sans aucun appareil spécial; il suffit d'agiter dans une carafe un mélange d'ẹau et d'éther ou de chloroforme, puis de séparer par une simple décantation la matière en excès qui surnage au-dessus de l'eau, si c'est de l'éther, et s'accumule au fond, si c'est du chloroforme.

En placant alors une cloche au-dessus de la plante plongée dans l'eau anesthésique, il sera facile de constater par les moyens ordinaires 
qu'elle n'absorbe plus d'acide carbonique et n'émet plus d'oxygène. Elle reste cependant parfaitement verte et ne paraît pas souffrir.

Bien plus, elle respire alors à la maniere des animaux, e'est-ì-dire en absorbant de l'oxygène et en exhalant de l'acide carbonique. C'est là une respiration véritable, marquée auparavant par le phénomène prédominant de l'assimilation du carbone et l'exhalation d'oxygène.

Voulez-vous maintenant réveiller votre plante pour vous convaincre qu'elle vit toujours, placez-la dans une eau non éthérée, et elle recommencera à s'assimiler de l'acide carboni. que et à dégager de l'oxygène sous l'influence des rayons solaires.

On peut aller plus loin encore et s'attaquer à un des phénomènes les plus intimes de la vie végétale, les fermentations.

La fermentation alcoolique du jus de la vigne ou du moût de la bière en offre des exemples bien connus. Ces fermentations sont produites par une sorte de petit champignon microscopique, ba levûre du vin, ou la levûre de la bière. 
Ce champignon décompose la matière sucrée pour s'en nourrir; il la dédouble en alcool qui reste dans la liqueur, et en acide carbonique qui, grâce à son état gazeux, peut s'échapper dans l'atmosphère.

Eh bien, plongez la levûre de bière avec une matière sucrée dans un appareil convenablement préparé, contenant de l'eau éthérée comme tout à l'heure, elle ne fermentera plus. Elle dort et ne sent plus la présence du sucre qui doit la nourrir. Quand votre conviction sera faite, retirez cette levûre, jetez-la sur un filtre pour la laver à l'eau ordinaire, et mettez-la ensuite dans une autre eau que l'éther n'a pas rendue soporifique, elle fermentera bientôt.

Mais si vous examinez la matière sucrée qui est restée avec la levûre de bière dans l'eau éthérée, vous y constaterez un phénomène singulier. Vous aviez mis du sucre de canne, vous retirez du sucre de raisin qui possède sans doute la même composition en poids, mais avec un autre groupement moléculaire.

Cette transformation bien connue est produite par un ferment inversif non organisé, qui 


\section{LA SENSIBILITÉ.}

accompagne dans la levùre de bière le fermentchampignon organisé dont nous avons seul parlé jusqu'ici. En effet, ce ferment-champignon n'est pas capable de s'assimiler le sucre de canne en nature; il faut que ce sucre soit digéré et transformé en sucre de raisin, exactement d'ailleurs comme cela se passe dans notre propre intestin. Le ferment-champignon a donc à côté de lui, dans la levûre même, une sorte de domestique donné par la nature pour opérer cette digestion à son profit, c'est le ferment inorganisé inversif. Ce ferment est soluble, ce n'est plus une plante, et comme il n'est pas organisé et qu'il n'a pas de sensibilité, il ne s'est pas endormi sous l'action de l'éther, et il a continué à remplir sa tâche, sans savoir que le sommeil de son maitre le rendait pour le moment inutile.

Puisque les animaux et les plantes possèdent tous une même sensibilité révélée par l'action des anesthésiques, il faut que cette sensibilité réside dans quelque chose de matériel, dans une substance qui se trouve chez tous ces êtres.

Pour atteindre ce siége de la sensibilité, il 
faut d'abord savoir que tous les tissus organiques, animaux ou végétaux, sont uniformément composés de cellules microscopiques infiniment petites, qui constituent le véritable siége de la vie et des phénomènes vitaux élémentaires.

C'est là que résident en réalité toutes les propriétés qui se manifestent ensuite dans les tissus organiques, simples agglomérations de ces individus cellulaires.

C'est dans ces cellules qu'est le siége de la sensibilité. Il s'y trouve une matière protéique, le protoplasma, qu'un naturaliste anglais, Th. Huxley, a nommé avec raison la base physique de la vie ${ }^{1}$. Cette matière se trouve partout, élément de la cellule dans les êtres complexes formant à elle seule l'être tout entier, lorsque celuici est réduit au dernier degré de simplicité. On trouve de ces êtres protoplasmiques même au fond des mers, êtres bizarres, dont on ne peut dire s’ils sont animaux ou végétaux, car ils n'ont aucune forme déterminée et peuvent les

1. Huxley, Les sciences naturelles el les problèmes qu'elles font surgir. Paris, 1877, p. 167. 
prendre toutes successivement. Huxley en a trouvé, à un millier de mètres au-dessous de la surface de l'Océan, un type fort curieux qu'il a nommé Bathybius Hæckelii, et Hæckel a même fait de ces êtres étranges un règne nouveau, celui des protistes.

Ce protoplasma, qui constitue seul certains protistes, se trouve dans toutes les cellules animales ou végétales; sous l'influence de l'éther, la cellule perd sa transparence, prend une légère opacité comme la vapeur d'eau qui se dépose sur un globe de verre; puis quand l'action de l'éther a cessé, le protoplasma, sans doute, redevient fluide, à peu près comme lil vapeur déposée sur le galobe de verre à l'état vésiculeux lui laisse de nouveau sa transparence en s'évaporant.

La sensibilité reparaît alors. On peut done croire que c'est dans cette substance primordiale protoplasmique que réside l'irritabilité ou la sensibilité initiale de l'être. Si l'unité da protoplasma établit l'unité physiologique des deux règnes organiques, en leur donnant à tous les deux un substratum de sensibilité, celi 
n'empêche pas que chacun ne réagisse suivant sa nature propre, et il est bien clair que le végétal fixé au sol et dépourvu de fibres motrices ne pourra pas réagir en s'enfuyant comme la plupart des animaux.

De là les différences qui séparent les êtres si variés de la nature.

Mais ces différences ne sont pas incompatibles avec l'unité qu'on remarque dans les phénomènes fondamentanx de la vie, parmi lesquels la sensibilité doit occuper le premier rang.

Ainsi la sensibilité est en quelque sorte le point de départ de la vie; elle est le grand phénomène initial d'où dérivent tous les autres, aussi bien dans l'ordre physiologique que dans l'ordre intellectuel et moral.

Association française pour l'avancement des Sciences. Session de Glermont-Ferrand. 1876. 


\section{ÉTUDES PHYSIOLOGIQUES}

SUR QUELQUES POISONS AMERIGAINS

\section{LE G U.RARE}

I

Les poisons peuvent être employés comme agents de destruction de la vie ou comme moyens de guérison des maladies; mais, outre ces deux usages bien connus de tout le monde, il en est un troisième qui intéresse particulièrement le physiologiste. Pour lui, le poison devient un instrument qui dissocie et analyse les phénomènes les plus délicats de la machine vivante, et, en étudiant attentivement le mécanisme de la mort dans les divers empoisonnements, il s'instruit par voie indirecte sur le mécanisme 
physiologique de la vie. Telle est la manière dont j'ai envisagé depuis longtemps l'action des substances toxiques ${ }^{1}$, et suivant laquelle je voudrais considérer ici les effets singuliers produits par quelques poisons américains encore peu connus.

Je commencerai ces études physiologiques par l'histoire du curare, le premier de ces poisons qu'il m'a été donné de soumettre à des investigations expérimentales.

Le curare ${ }^{2}$ est une substance dont se servent certaines peuplades sauvages de l'Amérique du Sud pour empoisonner leurs flèches, d'où le nom de poison de flèches qui lui a aussi été donné. Toutefois, la dénomination de poison de flèches comprenant des agents vénéneux très-divers, nous conserverons le nom de curare, généralement admis en Europe, pour désigner un poison américain qui est décrit dans les ré-

1. Voy. mes Lecons sur les effets des substances toxiques et médicamenteuses. Paris, 1856.

2. Encore nommé woorara, voorara, worari, wourari, wouraru, wurali, urari, ourari, ourary, etc., ou simplement veneno. 
cits des voyageurs, et qui se caractérise d'ailleurs par ses effets physiologiques, ainsi qu'on le verra plus loin.

Le curare est connu depuis la découverte de la Guyane par Walter Raleigh, en 1595. Raleigh, le premier, rapporta ce poison en Europe, sur des flèches empoisonnées, sous le nom de curari.

Beaucoup d'anciens voyageurs ont jugé à propos d'orner l'histoire du curare d'une foule de récits plus ou moins fabuleux, que nous devons passer sous silence pour ne nous arrêter qu'aux renseignements qui ont un caractère scientifique.

Dans un voyage fait en Amérique de 1799 à 1804, M. de Humboldt a pu assister à la fabrication du curare. C'est une sorte de fête comparable à celle des vendanges, la fiesta de las juvias. Les sauvages vont chercher dans les forêts les lianes du venin (juvias), après quoi ils font fête et s'enivrent avec de grandes quantités de boissons fermentées que les femmes préparent en leur absence. "Pendant deux jours, dit M. de Humboldt, on ne rencontre que 
des hommes ivres..." Lorsque tout dort dans l'ivresse, le maître du curare, qui est en même temps le sorcier et le médecin de la tribu, se retire seul, broie les lianes, en fait cuire le suc et prépare le poison. D'après ce qu'il a vu, M. de Humboldt admet que la composition du curare est exclusivement végétale, et que la propriété vénéneuse qu'il renferme est due à une plante de la famille des strychnées.

MM. Boussingault et Roulin, qui ont visité l'Amérique du Sud vingt-cinq ans plus tard, ont émis la même opinion.

Mais Ch. Watterton, qui parcourut en $181^{\circ}$ les contrées de Démérary et d'Essequibo, fait entrer dans la préparation du curare, outre les substances végétales, des fourmis venimeuses de deux espèces et des crochets de serpents broyés.

De même M. Goudot, qui a habité le Brésil pendant dix années, regarde le suc de liane épaissi comme jouant simplement le rôle d'un excipient dans lequel on introduit ensuite du venin de serpent. A son retour en France en 1844, il a remis à M. Pelouze, qui me l'a communiquée, 
une note sur la préparation du curare, que je crois utile de transcrire ici.

" Le curare est préparé par quelques-unes des tribus les plus reculées qui habitent les forêts qui bornent le Haut-Orénoque, le RioNegro et l'Amazone, et qui, toutes ou presque toutes, sont anthropophages....

“ La manière de préparer le curare varie dans chacune des tribus où il se fabrique, et celui qui est réputé le plus actif vient des nations voisines de l'empire du Brésil.

“ Le procédé employé par les Indiens du Mesaya, qui ne sont éloignés que de vingt journées de la frontière de la Nouvelle-Grenade, est le seul à peu près connu, et encore ne l'est-il que très-imparfaitement, car ces Indiens en font un grand secret, et il n'y a que leurs devins qui aient l'art de le préparer.

“ Ces hommes, qui sont en même temps les prêtres et les médecins ou guérisseurs de sor'ts, emploient pour la préparation du poison une liane nommée curari, d'où le non de curare donné au poison. Cette liane, coupée en troncons et broyée, donne un suc laiteux abondant cllaude bernard. 
et très-âcre. Les tronçons écrasés sont mis en macération dans de l'eau pendant quarantehuit heures, puis on exprime et on filtre soigneusement le liquide, qui est soumis à une lente évaporation jusqu'à concentration convenable. Alors on le répartit dans plusieurs petits vases de terre (figure 2), qui sont eux-mêmes

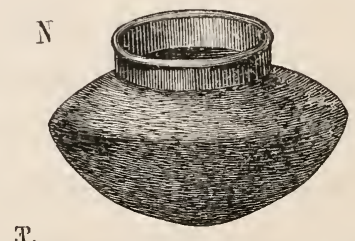

Fig. 2. Pot dans lequel s'opère la concentration du curare.

placés sur des cendres chaudes, et l'évaporation se continue avec plus de soin encore.

“ Lorsque le poison est arrivé à la consistance d'extrait mou, on y laisse tomber quelques gouttes de venin recueilli dans les vésicules des serpents les plus venimeux, et l'opération se trouve achevée lorsque l'extrait est parfaitement sec. »

Dans la relation d'une Expédition dans les parties centrales de l'Amérique du Sud, faite de 
1843 à 1847 sous la direction de M. F. de Castelnau, il est encore fait mention de la composition du curare. Les auteurs de cette relation reviennent à l'opinion de MIM. de Humboldt, Boussingault et Roulin, savoir que le curare est un poison végétal; mais ils assurent en outre que les Indiens ne mettent aucun secret dans cette préparation.

Enfin le dernier voyageur qui, à ma connaissance, ait écrit sur le curare, M. Émile Carrey, met tout le monde d'accord. Suivant lui, chez toutes les tribus, le curare aurait pour base un poison végétal identique : seulement il est des Indiens qui préparent le curare sans mystère et en y employant simplement les plantes actives, tandis que d'autres y ajoutent des substances plus ou moins singulières et entourent la fabrication de pratiques plus ou moins bizarres; mais ce serait par superstition ou par pur charlatanisme que les maîtres du curare de certaines tribus en agiraient ainsi, afin d'augmenter le prestige de leur puissance ou de cacher la composition du poison aux étrangers.

Les Indiens se servent du curare pour em- 


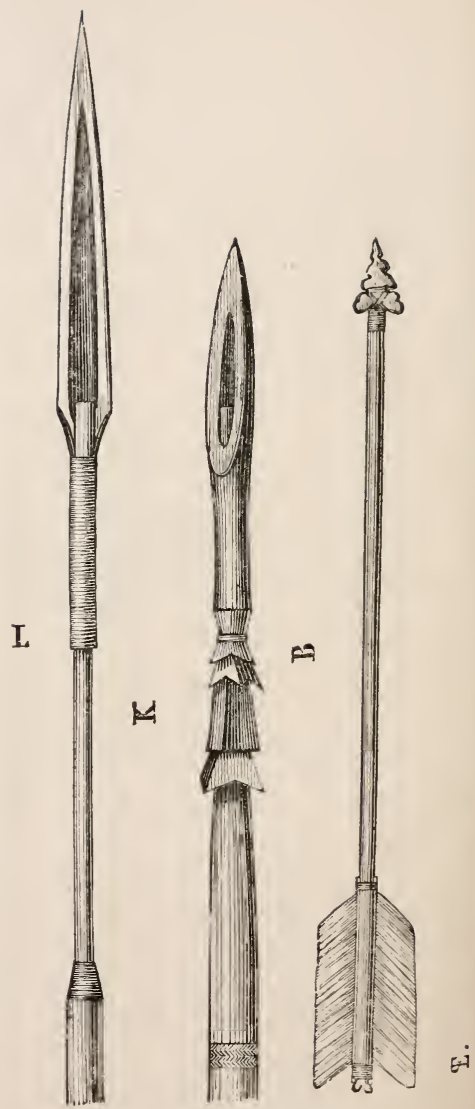

Fig. 3. Flèche de chasse'.

Fig. 4. Flèches de guerre ${ }^{2}$.

1. Le dard est mobile.

2. L, K, flèches taillées dans les os d'animaux; B, flèche dont la pointe est formée d'une lame de silex. 


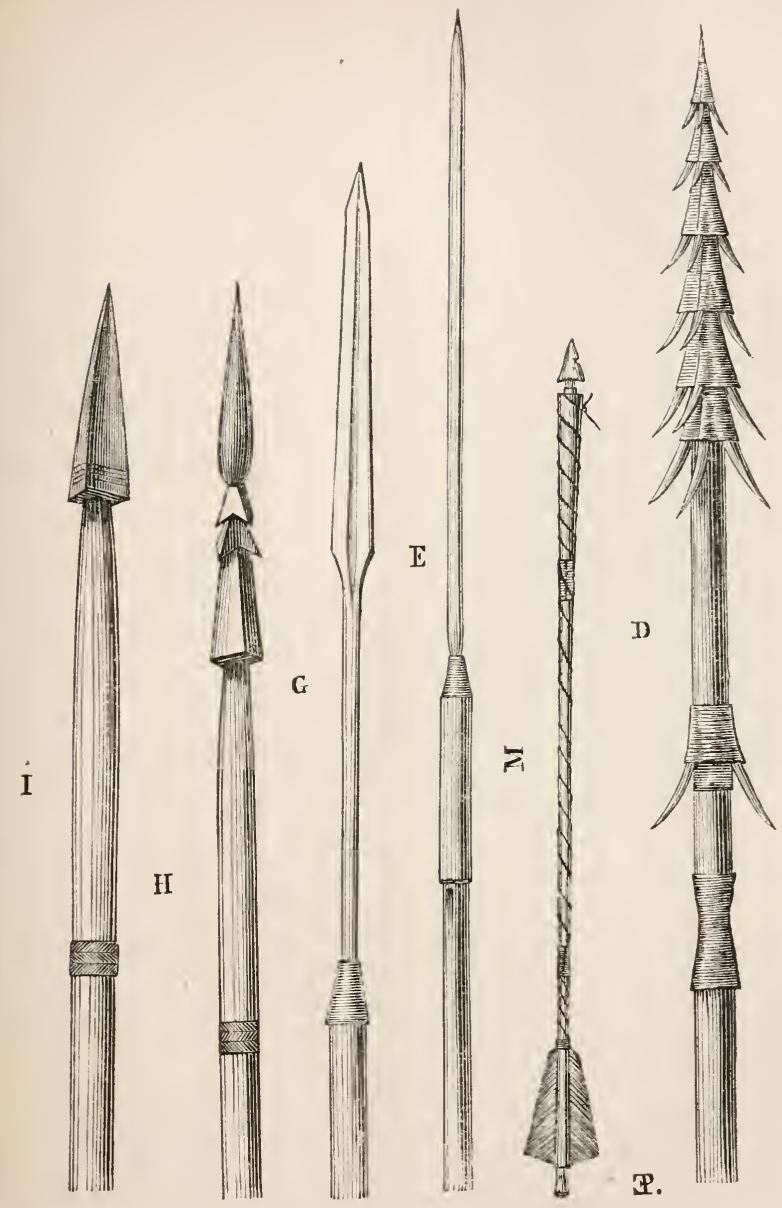

Fig. 5. Flèches de guerre ${ }^{1}$.

1. E, II, G, I, M, flèches dont l'extrẻmitẻ est taillée dans du bois très-dur; D, flèche apportẻe de Polynésie : autour de la flèche en bois de fer sont fixées, en sens inverse, des épines qui empêchent de retirer l'arme de la blessure. 
poisonner leurs flèches de chasse ou leurs flèches de guerre.

Les flèches de chasse (fig. 3), destinées à être lancées au moyen d'un are, sont pourvues d'un dard mobile; celles qui doivent être lancées au moyen d'une sarbacane sont très-petites, et ne forment en quelque sorte qu'un simple dard en bois de fer très-effilé et muni d'une pointe trèsaiguë qui porte le poison.

Les flèches de guerre (fig. 4 et 5) ont un dard fixe très-acéré, formé par des os d'animaux ou par du bois très-dur; quelquefois le dard est garni d'épines disposées en sens inverse, de manière à empêcher le trait de sortir de la blessure.

Outre ces armes toutes préparées, les Indiens ont encore leur provision de curare, qu'ils tiennent renfermée dans des petits pots de terre cuite ou dans des calebasses.

Le poison américain nous parvient en Europe sous ces trois formes. On ne peut se le procurer que par l'entremise des voyageurs; il n'existe pas dans le commerce européen, et les Indiens en font l'objet d'un échange, soit entre 
eux, soit avec les étrangers. "Les. Indiens de Mesaya, dit M. Goudot, une des tribus les plus féroces, préparent le curare et en font un commerce d'échange avec les habitants de la frontière de la Nouvelle-Grenade, qui, bravant les fièvres et les dangers de toute espèce, se basardent à pénétrer jusqu'au fond des forêts qu'ils habitent, et leur portent des haches, des couteaux, des ciseaux, des aiguilles et quelques étoffes de coton grossier. Ils reçoivent en payement du poison, de la cire d'abeilles presque aussi blanche que celle de Cuba, des fécules colorantes et du vernis qui peut être comparé à celui du Japon. ”

Le curare contenu dans les petits pots de terre cuite et dans les calebasses est un extrait noir à cassure brillante, présentant assez bien l'aspect de l'extrait du jus de réglisse noir de nos droguistes.

Le principe actif du poison est soluble dans l'eau, dans le sang et dans toutes les humeurs animales; mais il est mélangé de beaucoup d'impuretés qui restent en suspension dans le liquide, et où le microscope fait reconnaître en 
grande partie des débris de végétaux. Le vrai curare paraît conserver son activité d'une manière indéfinie, même à l'état de solution dans l'eau. J'en conserve ainsi depuis plus de dix ans qui semble n'avoir rien perdu sensiblement de ses propriétés toxiques, bien qu'il se soit produit des moisissures en grandé quantité à la surface du liquide. Comme l'eau, le sang et les humeurs animales, l'alcool dissout le venin curarique; l'éther et l'essence de térébenthine au contraire le précipitent. MM. Boussingault et Roulin ont préparé, sous le nom de curarine, le principe actif du curare. Toutefois le corps qu'ils ont obtenu n'est point cristallisable et défini; la curarine est une substance d'apparence cornée, très-hygrométrique, très-soluble dans l'eau et dans l'alcool.

Les caractères qui viennent d'être indiqués, de même que l'inaltérabilité du curare à l'ébullition et aux agents chimiques, ne sauraient permettre aucune induction sur la nature animale ou végétale du poison. En effet, c'est par erreur que l'on a cru jusqu'ici que tous les agents toxiques animaux se distinguaient des agents 
toxiques végétaux par une altérabilité plus grande; le venin de crapaud, par exemple, résiste à l'ébullition et se dissout dans l'alcool et l'éther. Il faudrait donc, pour résoudre la question de la composition du curare, saisir sur place l'agent réellement actif et le débarrasser de tous les ingrédients inutiles. Jusqu'ici les voyageurs, il est vrai, nous ont fourni le curare, mais avec lui ils ne nous ont rapporté que des récits et des descriptions contradictoires' de procédés de préparation. Aucun n'a essayé sur les lieux d'expérimenter par lui-même, pour savoir quelle était réellement la plante vénéneuse qui le constituait, afin de la caractériser et de la rapporter en Europe.

Le curare, à l'égal de beaucoup d'autres poisons énergiques, entrera certainement dans le domaine de la médecine; mais il serait nécessaire pour cela d'en connaître exactement la composition dans un temps assez rapproché. En effet, M. Émile Carrey nous apprend, dans l'intéressante relation de son voyage, que beaucoup de peuplades indiennes ont déjà renoncé à l'arme empoisonnée de l'homme primitif pour 
la remplacer par l'arme à feu de l'homme civilisé. Les flèches empoisonnées et le curare ne se trouvent plus aujourd'hui que chez les tribus les plus farouches de l'Amérique du Sud, et il pourrait bien se faire que d'ici à un demisiècle l'usage de ce poison et les procédés de préparation fussent complétement perdus.

Quant à son action sur les êtres vivants, le curare a toujours été représenté comme un poison violent dès qu'on l'introduit en contact avec le sang au moyen d'une plaie, mais inoffensif lorsqu'il est avalé et déposé dans les voies digestives. Les chairs des animaux tués par le curare sont en effet bonnes à manger et ne déterminent aucun accident.

On a dit que le curare était un poison aussi bien pour les végétaux que pour les animaux; cela est inexact. J'autres ont admis, sur la foi des récits, que les exhalaisons de curare sont vénéneuses. Vers le milieu du siècle dernier, La Condamine racontait que la cuisson du poison était confiée à une vieille femme : si cette femme mourait, le curare était jugé de bonne qualité; si elle ne mourait pas, on la battait de 
verges. II. Émile Carrey, avec sa verve naturelle, nous a décrit des pratiques analogues dont il avait entendu parler.

Comme on le voit, l'esprit s'est plu à entourer de merveilleux l'histoire de ce poison, dont l'origine et l'action étaient mal connues. Ici notre tàche sera de dépouiller les faits de toutes les interprétations mystérieuses pour n'admettre que ce que l'expérience nous prouvera directement; mais peut-être trouvera-t-on qu'on n'y aura rien perdu, et que les vérités scientifiques, quand nous pouvons les entrevoir, ne sont pas moins merveilleuses que les créations romanesques de notre imagination.

\section{II}

En 1844, je reçus de M. Pelouze des flèches empoisonnées ainsi que du curare qui avait été acheté par M. Goudot chez les Indiens Anda= quies au mois d'août 1842 . 
En 1848, un jeune Brésilien qui suivail mes cours, le docteurEdwards, me donna du curare que l'on retira d'une calebasse en l'exposant à la chaleur pour ramollir et extraire le poison qui en tapissait les parois.

Plus tard, j'ai expérimenté avec du curare qui nous avait été rapporté à M. Magendie et à moi par M. Émile Carrey, et qui provenait des bords de l'Amazone, avec du curare du Venezuela que m'avait remis M. Rayer, et avec du curare de Para dont M. Boussingault m'avait fait part.

J'ai constaté pour tous ces curares de diverses provenances des effets toxiques tout à fait semblables, sauf peut-être des nuances dans l'intensité du poison qu'il serait difficile de bien caractériser.

Un des faits qui paraît avoir le plus frappé tous ceux qui ont parlé du curare est l'innocuité de ce poison dans les voies digestives. Les Indiens, en effet, se servent du curare comme poison sous la peau et comme médicament dans l'estomac. J'ai entendu souvent raconter à M. Boussingault qu'il avait connu dans son 
voyage en Amérique un général colombien atteint d'épilepsie, qui, pour éviter les accès de sa terrible maladie, avalait des pilules assez volumineuses de curare. Les expériences sur les animaux ont confirmé les observations faites sur l'homme. On peut mélanger aux aliments d'un chien ou d'un lapin du curare en quantité beaucoup plus considérable qu’il ne serait nécessaire pour l'empoisonner par une plaie, et cela sans que l'animal en éprouve aucun inconvénient.

Toutefois il ne faudrait pas croire qu'il y ait là une propriété merveilleuse particulière au curare. C'est une simple question de dose et de rapidité de l'absorption.

Je me suis assuré par des expériences nombreuses que chez les jeunes animaux à jeun (mammifères et oiseaux), lorsque l'absorption intestinale est devenue plus active, le curare ne peut plus être aussi impunément introduit dans l'estomac, de sorte que cela se réduit simplement à dire qu'il faut des quantités beaucoup plus grandes de curare pour agir par les voies digestives que par une piqûre sous-cutanée.

CLAUDE BERNARD. 
C'est un cas commun, à des degrés divers, à beaucoup d'autres substances toxiques et médicamenteuses; la différence s'explique physiologiquement par la propriété que présentent les substances non cristalloïdes d'être absorbées très-lentement à la surface des membranes muqueuses.

Mais nous n'avons pas à nous arrêter à ces particularités qui concerneraient l'histoire thérapeutique du curare : je me hâte d'arriver à l'empoisonnement par piqûre, qui fait pénétrer rapidement le venin dans le sang, et amène la mort avec un cortége de symptômes particuliers que nous avons pour objet d'examiner et d'expliquer dans cette étude.

Le curare, introduit dans les tissus vivants à l'aide d'une flèche ou d'un instrument empoisonné, détermine la mort d'autant plus rapidement que le venin pénètre plus vite dans le sang. C'est pourquoi la mort est plus prompte quand on emploie une solution de curare au lieu de poison sec. Le degré de vitalité des animaux et la rapidité de la circulation qui en est la conséquence agissent dans le même sens. 
C'est ce qui fait que les animaux vigoureux sont plus faciles à empeisonner que les animaux languissants, et que, toutes choses égales d'ailleurs (taille de l'animal, dose du poison), les animaux à sang chaud meurent plus vite que les animaux à sang froid, et parmi les premiers les oiseaux plus vite que les mammifères.

La plaie empoisunnée par le curare n'est le siége d'aucune douleur ni d'aucune irritation particulière, le venin ne possède par lui-même aucune propriété caustique, de sorte que si la piqûre a été rapide, l'animal est empoisonné sans s'en apercevoir.

M. Boussingault m'a dit que, lorsque les Indiens blessent des oiseaux à la chasse avec les petites fièches qu'ils lancent à l'aide d'une sarbacane, et dont la pointe est acérée comme celle d'un aiguille, il arrive souvent que l'animal ne sent pas la blessure et qu'il meurt sur place en une minute ou deux.

Il n'en est pas ainsi quand on emploie de plus grandes flèches sur des animaux qui fuient; néanmoins la paralysie due à l'action du poison arrive assez vite pour que l'animal s'arrête et 
n'échappe jamais au chasseur. Watterton raconte qu'en traversant les terres qui séparent l'Essequibo du Démérary, lui et ses compagnons rencontrèrent une troupe de sangliers. Un Indien banda son arc et frappa l'un d'eux d'une flèche empoisonnée; elle entra dans la mâchoire et se rompit. Le sanglier fut trouvé mort à cent soixante-dix pas du lieu où il avait été frappé, et leur fournit un souper succulent.

Les symptômes de la mort par le curare offrent un aspect caractéristique sur lequel s'accordent tous les observateurs.

On ne pourrait guère constater ces symptômes chez les petits oiseaux, dont la mort a lieu parfois en quelques secondes; mais chez les oiseaux plus gros, chez les mammifères et chez les animaux à sang froid, la mort arrive dans un espace de temps qui varie en général entre cinq et douze minutes quand on a employé un excès de poison. Je rapporterai seulement trois ou quatre exemples; ils seront l'expression exacte de ce que j'ai toujours vu se reproduire dans les expériences en quelque 
sorte innombrables que j'ai répétées depuis vingt ans.

A l'aide d'une petite flèche empoisonnée, j’ai fait sur le dos d'un lapin une piqûre si peu douloureuse qu'il n'en a pas pour cela interrompu son repas; mais après deux ou trois minutes l'animal a cessé de manger et est allé se placer dans un coin du laboratoire : il s'est tapi contre le mur et a baissé ses oreilles sur son dos, comme s'il eût voulu dormir. Puis il est resté parfaitement tranquille et peu à peu s'est affaissé; ses jambes ont d'abord cédé en même temps que la tête a fléchi; enfin il est tombé sur le flanc complétement paralysé. Après six minutes, à partir du moment de la piqûre, l'animal était mort, c'est-à-dire que la respiration avait cessé.

Un jeune chien piqué à la cuisse avec un instrument empoisonné s'aperçut à peine de sa blessure; il courait et sautait comme de coutume, mais au bout de trois ou quatre minutes l'animal se coucha sur le ventre comme s'il eût été fatigué; il avait conservé toute son intelligence et ne semblait nullement souffrir; seule- 
ment il répugnait au mouvement. Bientôt le chien posa sa tête par terre entre ses deux jambes de devant, comme s'il eût été encore plus fatigué et qu'il eût voulu s'endormir. Cependant ses yeux restaient toujours ouverts et tranquilles en même temps que son corps s'affaissait sur lui-même; l'animal était alors complétement paralysé. Bientôt les yeux devinrent ternes, les mouvements respiratoires cessèrent, et l'animal était mort huit minutes après la piqûre empoisonnée.

Les grenouilles, les crapauds et les couleuvres meurent avec des symptômes semblables.

Les animaux ne manifestent aucune agitation ni aucune expression de douleur. Ils sont pris d'une paralysie progressive qui éteint successivement toutes les fonctions vitales. C'est là le caractère particulier de la mort par le curare.

Dans tous les genres de mort que l'on connaît, il y a toujours vers l'agonie des convulsions, des cris ou des râles indiquant une souffrance et une sorte de lulte entre la vie et la mort.

Dans la mort par le curare, rien de pareil; il n'y a pas d'agonie, la vie paraît s'éteindre. 
Tous les voyageurs qui ont vu périr des animaux par le curare décrivent la mort avec des symptômes pareils à ceux que nous venons d'indiquer. " La mort arrive, dit M. Carrey, comme si un fluide vital s'écoulait. "Watterton, qui nous a donné le plus de détails sur les effets du curare raconte que lorsqu'un oiseau est blessé à la chasse par une flèche empoisonnée, il reste environ trois minutes avant de tomber, mais que sa chute n'est précédée par aucun signe de douleur, qu'il y a seulement une sorte de stupeur qui se manifeste par une répugnance apparente au mouvement.

" Ayant empoisonné, dit-il, une jeune poule pleine de vie au moyen d'une piqûre faite à la cuisse avec une flèche empoisonnée, la poule n'en parut nullement incommodée. Pendant la première minute, elle marcha tranquillement; pendant la deuxième minute, elle resta calme et becqueta la terre. Moins d'une demi-minute après, elle ouvrit et ferma souvent le bec; sa queue était abaissée, et ses ailes tombaient presque à terre. A la fin de la troisième minute, elle était courbée, ne pouvant plus soutenir sa 
têie, qui tumbait, se relevait, et chaque fois tombait plus bas, comme celle d'un voyageur fatigué qui sommeille debout; ses yeux s'ouvraient et se fermaient. Au bout de cinq minutes, la poule était morte. "

Dans un autre exemple, il s'agit d'un paresseux dont la vie céda sans le moindre combat apparent, sans un cri ni un gémissement. C'était un aï ou paresseux à trois doigts; il appartenait à un naturaliste qui, voulant le tuer pour conserver sa peau, avait eu recours au curare. L'aï fut blessé à la jambe et mis sur le plancher, à peu de distance d'une table. Il s'efforça d'en atteindre le pied et s'y accrocha, comme s'il eût voulu monter; mais ce furent ses derniers efforts : sa vie s'éteignit rapidement, quoique graduellement.... D'abord une de ses jambes de devant lâcha prise et tomba de côté, incapable de se mouvoir; l'autre fit bientôt de même. Les membres antérieurs ayant perdu toute force, le paresseux se coucha lentement et mit sa tête entre ses jambes de derrière, qui tenaient encore à la table; mais lorsqu' elles furent atteintes à leur tour, il tomba à terre si doucement qu'on 
n'eût pas pu distinguer cette chute d'un mouvement ordinaire. Si l'on avait ignoré la circonstance de sa blessure, on n'eût jamais pensé qu’il succombait. La bouche était fermée; on n'y voyait ni ésume, ni salive. On n'observa ni tressaillement, ni altération visible de la respiration. Au bout de dix minutes, il fit un léger mouvement, et une minute après il était mort. "En un mot, dit Watterton, depuis le moment où l'action du poison commença à se montrer chez le paresseux, on aurait cru que le sommeil l'accablait."

Watterton nous donne encore le récit de la mort d'un homme empoisonné par le curare.

Deux Indiens couraient la forêt pour chercher du gibier. L'un d'eux prit une flèche empoisonnée et la lanca sur un singe rouge qui était au-dessus de lui, dans un arbre. Le coup était presque perpendiculaire. La flèche manqua le singe, et en retombant frappa l'Indien au bras, un peu au-dessus du coude. Il fut convaincu que tout était fini pour lui. " Jamais, dit-il à son camarade d'une voix entrecoupée et regardant son arc pendant qu'il parlait, jamais je ne ban- 
derai plus cet arc. " Ayant dit ces mots, il ôta la petite boîte de bambou contenant le poison qui était suspendue à son épaule, et, l'ayant mise à terre avec son are et ses flèches, il s'étendit auprès, dit adieu à son compagnon et cessa de parler pour toujours. " Ce sera une consolation pour les âmes compatissantes, remarque ailleurs Watterton, de savoir que la victime n’a pas souffert, car le wourali détruit doucement la vie. »

Ainsi toutes les descriptions nous offrent un tableau doux et tranquille de la mort par le curare. Un simple sommeil paraît être la transition de la vie à la mort. Cependant il n'en est rien; l'apparence extérieure est trompeuse. Cette étude sera donc propre à montrer combien nous pouvons être dans l'erreur relativement à l'interprétation des phénomènes naturels, tant que la science ne nous en a pas appris la cause et dévoilé le mécanisme. Si en effet, abordant maintenant la partie essentielle de notre sujet, nous entrons, au moyen de l'expérimentation, dans l'analyse organique de l'extinction vitale, nous verrons que cette mort, qui nous paraît 
survenir d'une manière si calme et si exempte de douleur, est au contraire accompagnée des souffrances les plus atroces que l'imagination de l'homme puisse concevoir.

\section{III}

Le corps d'un animal vivant est un assemblage admirable de particules, qui sont d'autant plus délicates et plus variées dans leurs propriétés physiologiques, que l'être occupe un rang plus élevé dans l’échelle de l’organisation. Or, il importe, pour la clarté de notre sujet, que nous descendions un instant dans cette machine vivante qui va devenir le théâtre des actions délétères que nous nous proposons de définir et d'expliquer.

Les manifestations vitales que nous apercevons au dehors ont une cause intérieure, cachée à nos regards. Elles ne sont toutes que des ré- 
sultantes de l'action réciproque et simultanée d'un grand nombre de particules organiques élémentaires, de même que dans la nature brute les phénomènes ne sont aussi que des résultantes complexes des propriétés des corps simples inorganiques. C'est donc dans les éléments organiques, c'est-à-dire dans les parties les plus déliées de l'organisme, que siégent les conditions intimes de la vie et de la mort. Le poison n'envahit jamais l'organisme d'emblée et dans sa totalité; mais il porte son action toxique et paralysante sur un élément organique essentiel à la vie. Ensuite il amène la dislocation de l'édifice vital par un mécanisme qui variera en raison de l'élément primitivement atteint, de la nature et de l'importance de ses rapports physiologiques avec l'ensemble des phénomènes de la vie.

La chimie connaît aujourd'hui soixante-dix corps simples environ, dont seize seulement entrent dans la composition de l'organisme vivant le plus compliqué, qui est celui de l'homme; mais ce n'est point en leur qualité de corps chimiquement simples que ces sub- 
stances viennent agir ici : elles se sont préalablement combinées et groupées sous l'influence de la force vitale, pour constituer les particules les plus ténues de notre organisme. Ces particules, bien que complexes chimiquement, sont élémentaires au point de vue physiologique en ce sens qu'elles sont douées de propriétés vitales simples et définies qui ne persistent pas après la division ou l'altération de l'élément. Telle est en quelques mots l'idée qu'on doit se faire des parties microscopiques de notre corps, auxquelles il convient de donner le nom d'éléments anatomiques ou peut-être mieux celui d'organismes élémentaires. En effet, les éléments anatomiques sont de véritables organismes élémentaires, et ce sont ces organismes élémentaires qui, par leur réunion et leurs groupements, sont ensuite appelés à constituer un organisme total d'autant plus complexe et d'autant plus élevé dans l'organisation que la variété physiologique de ses éléments se montre plus grande. Nous pouvons donc considérer que notre corps est composé par des millions de milliards de petits êtres ou individus vivants et 
d'espèce différente. Il en estqui sont libres comme les globules du sang; mais la plupart sont unis et soudés. Les éléments de même espèce se réunissent pour constituer nos tissus, et nos tissus se mélangent pour former nos organes; les éléments d'espèce différente se soudent entre eux afin de pouvoir réagir les uns sur les autres et concourir avec harmonie à un même but physiologique. Néanmoins, dans toutes ces réunions ou soudures, aucun élément ne se confond avec son voisin; ils s'unissent et restent distincts comme des hommes qui se donneraient la main. Chaque espèce d’éléments représente ainsi une véritable espèce d'individus qui dépend d'un tout auquel il est associé, mais qui a toujours son indépendance et sa vie propre, qui a sa manière particulière de se nourrir et d'être excité, qui a ses poisons spéciaux et sa manière spéciale de mourir. Enfin, comme on peut le dire d'un seul mot, chaque élément a son autonomie, mais autonomie inconsciente et enchâ̂née par un déterminisme absolu aux conditions physico-chimiques du milieu organique intérieur. 
A part les éléments organiques qu'on peut appeler passifs, parce que, par leur réunion, ils constituent la charpente osseuse du corps, ainsi que tous les tissus conjonclifs qui donnent la solidité, l'élasticité et la cohésion à nos organes, il existe deux autres classes d'éléments organiques qui nous manifestent une activité constante et nécessaire.

Dans la première classe, nous placerons tous les éléments organiques qui, sous la forme de vésicules ou de cellules soit libres, soit fixées ou agglomérées, constituent les tissus glandulaires, muqueux et épithéliaux. Les propriétés de ces éléments groupés en tissus se manifestent plus particulièrement dans l'accomplissement des phénomènes de la vie nutritive.

Nous placerons dans la seconde classe les éléments organiques qui, généralement sous la forme de fibres ou de tubes réunis ou soudés les uns aux autres, constituent les tissus musculaires et nerveux. En raison de leurs propriétés, ces derniers éléments président aux fonctions de sensibilité et de mouvement qui sont 
propres aux animaux et constituent les manifestations les plus élevées des êtres vivants.

L'objet de la physiologie générale est d'analyser chaque fonction et chaque acte de l'économie, afin de les ramener à leur élément organique.

Le phénomène de la respiration, malgré ses variétés apparentes, se réduit finalement pour tous les animaux à la propriété de l'élément ou globule sanguin qui, au contact de l'air, absorbe l'oxygène et exhale l'acide carbonique.

La digestion, avec les sécrétions qui y concourent, se ramène à l'élément glandulaire ou épithélial, qui, sous l'influence de certains excitants déterminés, laisse suinter un liquide qu'il a la propriété de préparer et d'accumuler en lui.

De même, quand nous voyons apparaître dans un animal un phénomène de sensibilité oı de mouvement, nous devons nous reporter par l'analyse physiologique aux propriétés des fibres nerveuses et musculaires qui constituent ses conditions élémentaires.

La fibre musculaire (fig. 6) représente lin 
tube microscopique à parois élastiques; ce tube est rempli d'une substance contractile, c'est-àdire d'une matière qui, pendant la vie, jouit de la propriété de se contracter sous l'influence

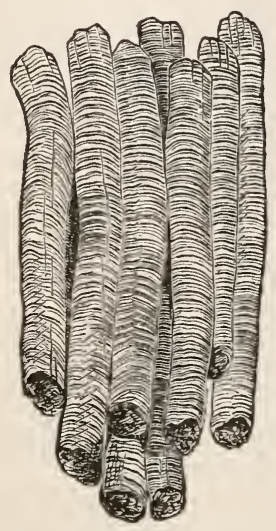

Fig. 6. Fibres musculaires '.

nerveuse de façon à raccourcir le tube musculaire et à entraîner dans son mouvement les parties auxquelles il est fixé.

Nous trouvons dans le système nerveux des éléments producteurs et conducteurs, les uns

1. Fort grossies au microscope (d'après Dalton). 


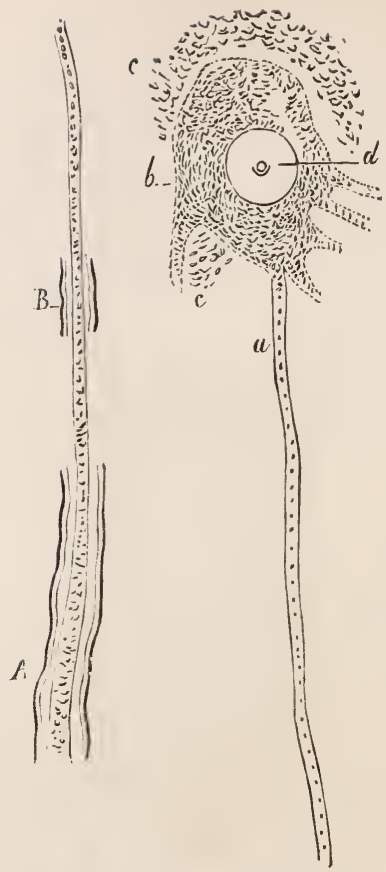

Fig. 7. Tubes nerveux ${ }^{1}$.

1. A, tube nerveux composé par ses trois éléments, l'enveloppe à double contour, la moelle nerveuse et le cylinder axis. Dans un point, l'enveloppe A a été rompue, et le cylinder axis est resté seül. - A côté, un fragment des enveloppes $\mathrm{B}$, qui ont été conservées. - $d$, noyau d'une cellule nerveuse cérébrale multipolaire. - $a$. Tube nerveux réduit à son axis; - $c, c$, granulations moléculaires entourant la cellule nerveuse (d'après R. Wagner). 


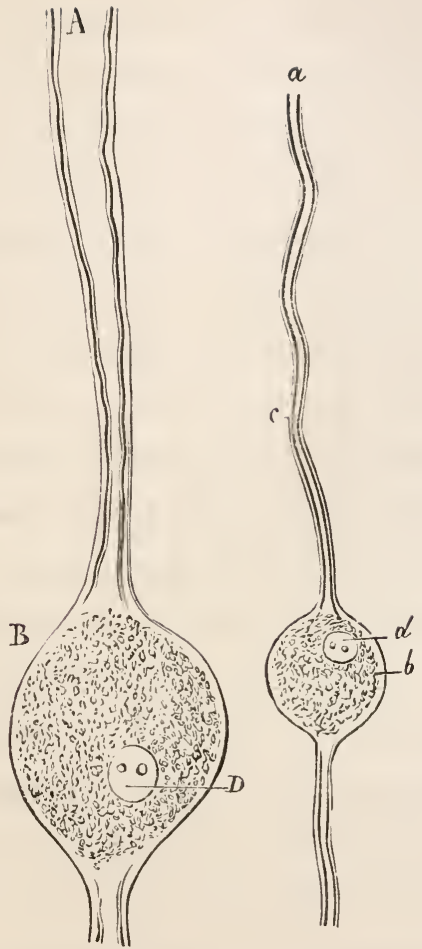

Fig. 8. Cellules nerveuses rachidiennes ${ }^{1}$.

1. A, tube nerveux gros, composé de ses trois parties; $\mathrm{B}$, cellule nerveuse; D, noyau de la cellule; $a$, petit tube nerveux; $b$, petite cellule; $c, d$, noyaux de la petite cellule. 
pour la sensibilité, les autres pour la motricité ( fig. 7 et 8). Les conducteurs nerveux représentent de véritables fils électriques organiques; ils sont constitués par un tube rempli d’une substance appelée moelle nerveuse, destinée à protéger un filament central. Ce filament est la partie physiologiquement essentielle du nerf, et qu'on appelle l'axe du cylindre nerveux ou le cylinder axis. Le tube nerveux sensitif s'unit au

- tube moteur au moyen d'un renflement nerveux appelé cellule nerveuse, et le tube moteur se termine dans la fibre musculaire en présentant une nouvelle intumescence particulière.

Tous ces éléments organiques qui composent notre corps sont d'une grande ténuité microscopique, car la grandeur en varie entre des centièmes et des millièmes de millimètres. On pourra par conséquent avoir une idée de leur nombre par leur masse, quand on saura que les cellules et les tubes nerveux, par leur réunion, forment le cerveau, la moelle épinière et les cordons nerveux, et que toutes les fibres musculaires ensemble constituent essentiellement la viande ou la chair qui représente la plus grande partie du 
poids du corps de l'homme et des animaux.

Quelles que soient la complication et la variété de nos opérations intellectuelles, de nos sentiments et de nos mouvements, ils ne sont jamais exprimés que par l'activité vitale de trois éléments organiques formant une chaîne à anneaux distincts, mais dont les propriétés sont cependant physiologiquement et hiérarchiquement subordonnés.

Ces trois éléments sont l'élément nerveux sensitif, l'élément nerveux moteur, et l'élément musculaire. Le point de départ de l'action physiologique se trouve dans l'élément nerveux sensitif ou intellectuel; sa vibration se transmet suivant son axe, et, arrivée à la cellule nerveuse, véritable relais, la vibration sensitive se transforme en vibration motrice. Cette dernière se propage à son tour dans l'élément nerveux moteur, et, arrivée à son extrémité périphérique, elle fait vibrer la fibre musculaire, qui, réagissant en vertu de sa propriété élémentaire, opère la contraction ou le mouvement.

Ces trois éléments organiques jouent ainsi le rôle d'excitant les uns par rapport aux autres; 
l'élément nerveux sensitif excite l'élément nerveux moteur, et l'élément nerveux moteur excite la fibre musculaire, d'où résulte finalement la contraction. Dans leur action d'ensemble, ces éléments ont des relations tellement connexes que, les uns sans les autres, ils n'auraient point de raison d'être. En effet, l'élément sensitif n'a pas de raison d'ètre sans l'élément moteur qui indique sa présence, et l'élément moteur n’aurait pas de raison d'être sans l'élément musculaire sur lequel son influence doit se manifester.

Toutefois, malgré cette connexion intime et nécessaire, chacun de ces trois éléments n'en reste pas moins indépendant et distinct organiquement. L'élément sensitif vit et meurt à sa manière, il a ses poisons qui lui sont propres. L'élément moteur peut vivre et mourir séparément, il a également ses poisons spéciaux. Enfin l'élément musculaire a de même des conditions de vie et de mort qui n'appartiennent qu'à lui.

Si cette indépendance organique est réelle pour la vie nutritive des éléments, elle n'est 
plus qu'une illusion au point de vue des manifestations vitales qu'ils doivent accomplir dans l'organisme. Ces manifestations n'étant qu'une résultante d'activités diverses, elles exigent le concours de toutes. Si l'un des trois éléments, sensitif, moteur et musculaire, vient à être supprimé, les deux autres continuent de vivre sans doute, mais ils n'ont plus de sens, de même qu'une phrase perd sa signification dès qu'un de ses membres vient à lui manquer.

La loi fondamentale de la vie est l'échange de matières continuel entre le corps vivant et le milieu cosmique qui l'entoure. De là résulte un véritable circulus ou tourbillon rénovateur du corps dont la rapidité mesure l'intensité de la vie. Les conditions des phénomènes vitaux ne sont absolument constituées ni par l'organisme, ni par le milieu; il faut le concours des deux. Malgré l'intégrité de l'organisme, la vie cessera, si le milieu est supprimé ou vicié; malgré la présence d'un milieu favorable, la vie s'éteindra, si l'organisme est lésé ou détruit.

Notre corps entier ou notre organisme n'est, nous le répétons, qu'un agrégat d'éléments or- 
ganiques, ou mieux d'organismes élémentaires innombrables, véritables infusoires qui vivent, meurent et se renouvellent chacun à sa manière. Cette comparaison exprime exactement ma pensée, car cette multitude inouïe d'organismes élémentaires associés qui composent notre organisme total existent, comme des infusoires, dans un milieu liquide qui doit être doué de chaleur et contenir de l'eau, de l'air et des matières nutritives. I es infusoires libres et disséminés à la surface de la terre trouvent ces conditions dans les eaux où ils vivent. Les infusoires organiques de notre corps, plus délicats, groupés en tissus et en organes, trouvent ces conditions, entourés de protecteurs spéciaux, dans notre fluide sanguin, qui est leur véritable liquide nourricier. C'est dans ce liquide, qui ne les imbibe pas, mais qui les baigne, que s'accomplissent tous les échanges matériels, solides, liquides ou gazeux, que leur vie exige; ils y prennent leurs aliments et y rejettent leurs excréments, absolument comme des animaux aquatiques. D'ailleurs la vie ne s'accomplit jamais que dans un milieu liquide. Ce n'est que 
par des artifices de construction que les organismes de l'homme, ainsi que ceux d'autres animaux, peuvent vivre dans l'air; mais tous les éléments actifs de leurs fonctions vivent sans exception, à la façon des infusoires, dans un milieu liquide intérieur. C'est pourquoi j’ai donné le nom de milieu intérieur organique au sang et à tous les liquides blastématiques qui en dérivent.

Le système circulatoiren'est autre chose qu'un ensemble de canaux destinés à conduire l'eau, l'air et les aliments aux éléments organiques de notre corps, de même que des routes et des rues innombrables serviraient à mener les approvisionnements aux habitants d'une ville immense. Les canaux veineux n'ont pas, à proprement parler, de rapports physiologiques actifs avec les éléments organiques; ils ne leur portent rien, ils ne fontqu'emmener le sang qui a servi à les nourrir; mais le système veineux présente une autre origine périphérique de la plus haute importance, car c'est par cette origine que le courant veineux, dont la direction est centripète, vient se répandre sur les diver- 
ses surfaces de l'organisme et puiser l'air dans les poumons, de l'eau et des aliments dans les intestins, ainsi que d'autres liquides interstitiels. Tous ces éléments constitutifs du milieu intérieur sont ensuite portés au cœur, centre du mouvement circulatoire. Ici commence le système artériel qui lance le sang dans une direction inverse à celle qui précède, c'est-à-dire du centre à la périphérie. Le sang ainsi poussé par le cœur dans les artères va se purifier en tout ou en partie de divers produits d'élimination et par des mécanismes divers, suivant les organismes; mais ce qu'il importe de savoir ici, c'est que le sang artériel est celui qui se dirige vers nos organismes élémentaires et qui leur distribue toutes les substances capables de réagir sur eux. Le sang artériel porte la vie aux éléments organiques, parce qu'il contient en dissolution de l'oxygène et les autres éléments d'un milieu organique propre à entretenir la vie ; mais le sang artériel peut aussi apporter la mort, s'il est introduit dans les voies circulatoires, c'est-à-dire dans le milieu intérieur organique, des substances qui l'ont vicié. Or c'est 
le cas qui se présente dans tous les empoisonnements.

Lorsqu'un animal est piqué par une flèche empoisonnée avec du curare, nous avons vu qu'il ne meurt qu'après un certain temps. Il y a en effet trois étapes nécessaires que le poison doit parcourir. Premièrement le poison doit être dissous dans la plaie par les humeurs animales qui s'y trouvent; deuxièmement, il doit pénétrer dans les veines et être porté jusqu'au cœur; troisièmement, il doit être amené en contact avec les éléments organiques au moyen du système artériel. Ce n'est point encore tout : il faut que la substance toxique s'accumule dans le sang par suite d'une disproportion qui doit s'établir entre l'absorption et l'élimination du poison. Tout cela demande, ainsi que nous le savons, un maximum de dix à douze minutes pour s'accomplir. Nous concevons maintenant que le curare puisse ne pas agir si, avant d'arriver au système artériel, il rencontre sur sa route quelque voie d'élimination rapide, ou s'il se trouvait, par un obstacle quelconque, retenu dans le système veineux. En effet dans ce cas 
le poison ne parvient pas jusqu'aux voies qui conduisent aux éléments organiques.

Trois ans après le retour de Watterton en Angleterre, Brodie fit quelques expériences qu'il importe de mentionner. On inocula du curare à la jambe d'un âne, et il mourut en douze minutes. Sur un autre âne, on inocula le même poison, et de la même manière, mais après avoir placé un bandage autour de la jambe au-dessus de l'endroit où l'inoculation avait été pratiquée. l'âne marcha librement, comme à l'ordinaire, et il continua à manger sans s'apercevoir de rien. Au bout d'une heure on délia le bandage, et dix minutes après la mort avait saisi cet animal. Ces expériences, qui sont imitées de celles que Magendie avait faites pour d'autres poisons et qui ont été bien souvent confirmées, s'expliquent physiologiquement d'une manière trèssimple : tant que le poison restait sous la peau de la jambe au-dessous de la ligature, ilne pouvait pas arriver au cœur, parce que cette ligature empêchait le sang veineux de passer et de l'y transporter. Le poison, avons-nous dit, n'est actif que lorsque, étant parvenu au cœur, il 
peut se répandre par les artères, et arriver ainsi à tous les éléments organiques; mais là encore nous pouvons, à l'aide d'un artifice expérimental, empêcher le poison de se généraliser. Si nous lions l'artère d'un membre par exemple, nous empêcherons le sang empoisonné d'être porté aux éléments organiques de ce membre, et nous leur conserverons la vie, tandis que tout le reste du corps aura ressenti les atteintes délétères de la substance toxique. En un mot, en arrêtant le poison dans les veines, on sauve tout l'individu; en arrêtant le poison dans les artères, on ne sauve que la partie du corps à laquelle l'artère oblitérée portait le sang.

Après cet exposé sommaire de quelques notions physiologiques qu'il était nécessaire de rappeler, revenons aux effets du poison améri cain. Nous aurons à rechercher d'abord sur quel élément organique particulier du corps il a porté son action toxique, et ì déterminer ensuite le mécanisme par lequel la mort de cet élément a pu amener la mort de tout l'organisme. 
Dans le mois de juin 1844, je fis ma première expérience sur le curare : j’insinuai sous la peau du dos d'une grenouille un petit fragment de curare sec, et j'observai l'animal. Dans les premiers moments, la grenouille allait et sautait comme avant avec la plus grande agilité, puis elle resta tranquille. Au bout de cinq minutes, les jambes de devant cédèrent, le corps s'aplatit et s'affaissa peu à peu. Après sept minutes, la grenouille était morte, c'est-à-dire qu'elle était devenue molle, flasque, et que le pincement de la peau ne déterminait plus chez elle aucune réaction vitale.

Je procédai alors à ce que j'appelle l'autopsie physiologique de l'animal.

Des mesures/sages, et que tout le monde approuve, empêchent de faire chez l'homme les 
autopsies avant qu'il se soit écoulé vingtquatre heures depuis le moment de la mort. Cette circonstance diminue considérablement l'importance scientifique des autopsies cadavériques. En effet, la vie ne cesse pas parce que tout notre corps est mort à la fois, mais seulement parce que un ou plusieurs de ses éléments organiques ont perdu leurs propriétés vitales. En faisant l'autopsie au moment même de la mort, on doit donc toujours rencontrer des éléments organiques qui ont perdu leurs propriétés physiologiques; mais d'autres qui les possèdent encore, et qui ne finissent par les perdre et par mourir à leur tour qu'à cause de la dislocation des fonctions nécessaires à leur existence. Quand on pratique l'autopsie vingtquatre heures après la mort, tous les éléments organiques sont éteints, rigides et froids. On ne trouve plus que des lésions chroniques qui nous font connaître les diverses métamorphoses pathologiques des tissus, mais qui ne nous expliquent en rien le mécanisme de la mort, car l'individu vivait quelques heures auparavant avec cette même lésion. Dans d'autres cas, 
on ne trouve rien, et on croit que la cause de la mort est insaisissable.

C'est ce qui nous serait arrivé, si nous eussions fait l'autopsie de notre grenouille le lendemain: nous aurions eu un cadavre empoisonné par le curare qui ne nous aurait offert aucune lésion, qu'il nous eût été impossible de distinguer sous aucun rapport du cadavre d'une grenouille morte d'une tout autre manière.

Il en est autrement, ainsi qu'on le verra, lorsqu'on fait l'autopsie physiologiquement, c'est-à-dire en ouvrant l'animal aussiôt après la mort. C'est là un avantage des plus importants que présente seule la pathologie expérimentale, car ce que la morale interdit de faire sur nos semblables, la science nous autorise à le faire sur les animaux. L'homme, qui a le droit de se servir des animaux pour ses usages domestiques et pour son alimentation, a également le droit de s'en servir pour s'instruire dans une science utile à l'humanité.

En ouvrant la grenouille empoisonnée (fig.9), je vis que son cœur continuait à battre. Son sang rougissait à l'air et présentait ses pro- 


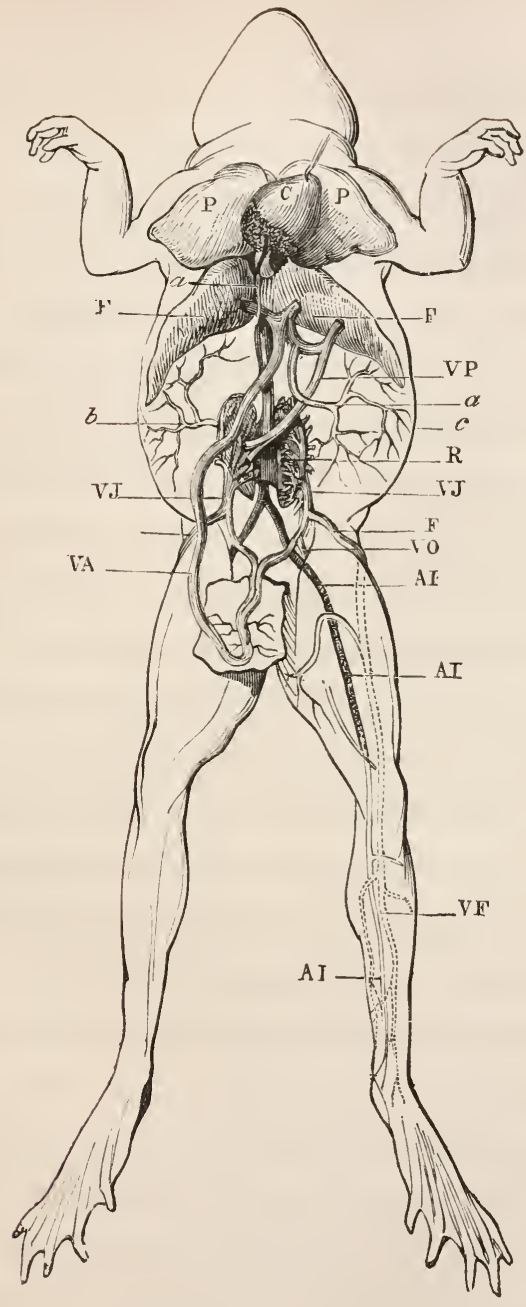

Fig. 9. Système vasculaire de la grenouille :

1. A, veine allant de la reine-cave au cour en traver- 
priétés physiologiques normales. Je me servis ensuite de l'électricité comme de l'excitant le plus convenable pour réveiller et provoquer la réaction physiologique des éléments nerveux et musculaires. En agissant directement sur les muscles, l'excitant électrique produisait des contractions violentes dans toutes les parties du corps; mais en agissant sur les nerfs euxmêmes il n'y avait plus aucune réaction. Les nerfs, c'est-à-dire les tubes nerveux qui les composent, étaient donc complétement morts, tandis que les autres éléments organiques des muscles, du sang, des muqueuses, etc., étaient très-vivants et conservaient encore leurs propriétés physiologiques pendant un grand nombre d'heures, ainsi que cela se voit surtout chez les animaux à sang froid.

Il est maintenant facile de comprendre que

sant le péricarde; PP, poumon; G. cœur; F, foie; VP, veine porte; $b c$, veines épiloïques; $\mathrm{R}$, reins; $\mathrm{VJ}$, veines de Jacobson; F, veine crurale; AI, artère iliaque allant constituer l'aorte au niveau du bord inférieur des reins; $\mathrm{VA}$, veines abdominales allant se rendre au foie; $\mathrm{AL}$, artère crurale; VF, veine fémorale. 
l'extinction vitale des éléments nerveux qui fon $\mathrm{t}$ contracter les muscles doive amener la mort de l'organisme tout entier par la cessation successive de tous les mouvements. L'arrêt des mouvements respiratoires produit particulièrement ce résultat en empêchant dans le milieu organique sanguin l'aération, qui est indispensable pour entretenir la vie de tous les éléments organiques qui nous composent. Si le cœur conserve encore ses mouvements, cela prouve, ainsi qu'on le savait déjà, qu'il n'est pas influencé par le système nerveux comme les autres muscles, ce qui lui permet d'être, suivant l'expression de Haller, l'organe primum vivens et l'organe ultimum moriens. En outre la démonstration de cette action nette et caractéristique du curare, qui tue l'élément nerveux et respecte l'élément musculaire, a résolu la question de ce qu'on appelait l'irritabilité hallérienne, en prouvant expérimentalement que lia propriété contractile du muscle est distincte de la propriété du nerf qui l'excite, puisque le poison parvient à les séparer immédiatement l'une de l'autre. 
Cette première expérience analytique faite sur la grenouille a ensuite été répétée de la même manière sur d'autres animaux plus rapprochés de l'homme et appartenant à la classe des oiseaux et des mammifères. J'ai constaté des résultats tout à fait semblables, et l'autopsie physiologique me montra que, comme chez la grenouille, l'élément nerveux moteur avait été seul atteint par le curare, tandis que les autres éléments organiques avaient conservé leurs propriétés physiologiques.

L'observation attentive des symptômes de l'empoisonnement sur les animaux élevés vint me révéler des particularités intéressantes relatives à la sensibilité et à l'intelligence.

Un chien d'une humeur douce avait été blessé par une flèche empoisonnée. D'abord l'animal ne s'en apercut pas : il courait, gambadait joyeusement comme à l'ordinaire; mais bientôt, comme s'il eût été fatigué, il se coucha sur le ventre, dans une attitude très-naturelle. Quand on appelait le chien, il répondait à l'appel; il se levait et venait, après des sommations réitérées et avec une sorte de lassitude. 
Peu de temps après, le chien ne pouvait plus se lever malgré ses efforts; il avait conservé toute son intelligence el ne paraissait nullement souffrir; seulement ses jambes, et particulièrement celles du train de derrière, n'obéissaient plus à sa volonté. Lorsqu'on parlait à l'animal, il répondait parfaitement bien par les mouvements de la tête, par l'expression des yeux et par l'agitation de la queue; mais un peu plus tard la tête tomba, l'animal ne pouvait plus la soutenir. Le chien était alors couché et respirait avec calme, comme un animal qui aurait reposé tranquillement; si on l'appelait, sa queue seule pouvait s'agiter, et ses yeux se tourner encore et sans aucune expression de souffrance, pour montrer qu'il entendait. Enfin les mouvements respiratoires cessèrent peu à peu, et les yeux étaient déjà devenus ternes et sans vie que des mouvements légers de la queue venaient témoigner que le chien entendait encore celui qui lui parlait.

Un autre chien d'une nature fẻroce, et cherchant à mordre tous ceux qui l'approchaient, fut piqué par une flèche empoisonnée. Pendant 
les premiers moments, l'animal farouche, blotti dans son coin, faisait entendre des grondements mêlés d'aboiements toutes les fois qu'on se dirigeait vers lui. Après six ou sept minutes, l'animal se coucha, ses jambes ne pouvaient plus le soutenir, et ses cris s'éteignirent, mais il n'en était pas moins furieux. Toutes les fois qu'on approchait, il montrait les dents et roulait des yeux flamboyants. Quand on lui présentait un bâton, il le mordait avec force et arce une rage silencieuse. Cette rage ne s'éteignit qu'avec la vie, et lorsque le chien ne pouvait plus la manifester par ses lèvres et par ses dents, elle était encore dans ses regards, qui, les derniers, exprimèrent sa furie.

Les deux expériences qui précèdent nous montrent que dans la mort par le curare l'intelligence n'est point anéantie; chacun de nos animaux a conservé son caractère jusqu'au bout, et si les manifestations caractéristiques ont disparu, ce n'est pas parce qu'elles se sont réellement éteintes, mais parce qu'elles se sont trouvées successivement refoulées et comme envahies par l'action paralytique du poison. En 
effet, dans ce corps sans mouvement, derrière cet œil terne, et avec toutes les apparences de la mort, la sensibilité et l'intelligence persistent encore tout entières. Le cadavre que l'on a devant les yeux entend et distingue ce que l'on fait autour de lui, il ressent des impressions douloureuses quand on le pince ou qu'on l'excite. En un mot, il a encore le sentiment et la volonté, mais il a perdu les instruments qui servent à les manifester : c'est ce que nous allons montrer en poussant plus loin notre analyse physiologique.

Rappelons-nous pour un instant que le curare ne peut exercer son action toxique qu'après avoir été porté par les artères et mis en contact avec nos éléments organiques. Rappelons-nous encore qu'en liant ou en obstruant une artère d'un membre ou d'une autre partic du corps, on peut ainsi préserver cette partie de l'empoisonnement qui envahira tout le reste de l'organisme. Or à l'aide de ce membre ou de cette partie réservée, ne fùt-ce même que d'une fibre musculaire, l'animal pourra manifester ce qu'il sent et montrer que son intelligence, qui 
avait été en quelque sorte saisie dans un cadavre, n'avait pas été abolie. Ces expériences analytiques se démontrent particulièrement bien chez les animaux à sang froid à cause de la persistance plus longue des propriétés élémentaires des tissus après l'arrêt de la circulation artérielle.

Sur une grenouille très-vivace j'ai intercepté le passage du sang artériel dans les jambes du train de derrière par la ligature des artères, en ayant grand soin de laisser intacts les nerfs qui font communiquer ces membres avec la moelle épinière (fig. 10). Après cette opération, la grenouille avait conservé toute son agilité, sautait et nageait comme à l'ordinaire. Alors je l'empoisonnai en lui insinuant un petit fragment de curare sous la peau du dos. Après cinq minutes, la grenouille s'affaissa, ses jambes de de. vant, ayant perdu leur ressort, s'écartèrent, et la mâchoire inférieure de l'animal reposait sur la table. Après sept ou huit minutes, la grenouille était morte et sans mouvement. Quand on pinçait la peau de la tête, du corps ou des paltes de devant, il n'y avait aucun mouvement 
ni aucune réaction vitale dans ces parties empoisonnées; mais la grenouille agitait aussitôt

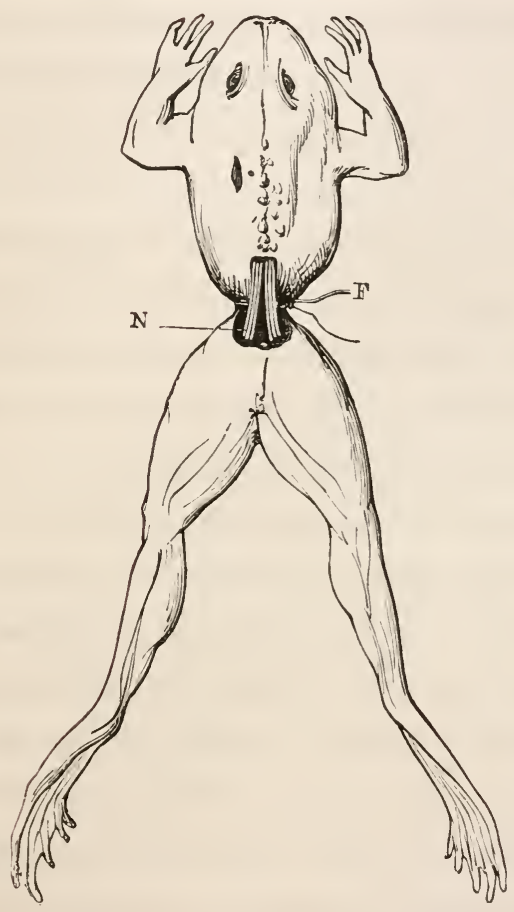

Fig. 10. Grenouille préparée pour l'expérimentation '.

avec violence ses deux pattes de derrière, qui

I. F, fil de la ligature; N, nerfs lombaires. 
avaient été préservées de l'empoisonnement par la ligature des artères. Ce résultat était constant même après les plus légères piqûres dans la partie du corps empoisonnée. Quand on mettait la grenouille dans l'eau et qu'on excitait une partie quelconque de son corps, elle nageait parfaitement avec ses deux jambes de derrière, qui poussaient devant elles le reste du corps complétement immobile, quoique sensible; mais non-seulement notre grenouille avait conservé la sensibilité dans le train antérieur de son corps paralysé par le poison, elle y avait encore conservé ses sens et sa volonté. En effet, si l'on couvrait le vase où l'on avait introduit la grenouille de manière à la placer dans l'obscurité, et si ensuite on faisait subitement pénétrer un rayon de soleil en déplacant le couvercle, on apercevait le troncon de la grenouille flasque et incliné en bas s'avancer volontairement vers le soleil à l'aide des deux jambes de derrière.

J'ai répété l'expérience très-souvent; elle a toujours réussi.

Si, au lieu des deux jambes, on n'en préserve 
qu'une de l'empoisonnement, le résultat est le même (fig. 11); seulement il n'y a qu'une jambe qui se meut quand on pince l'animal, et

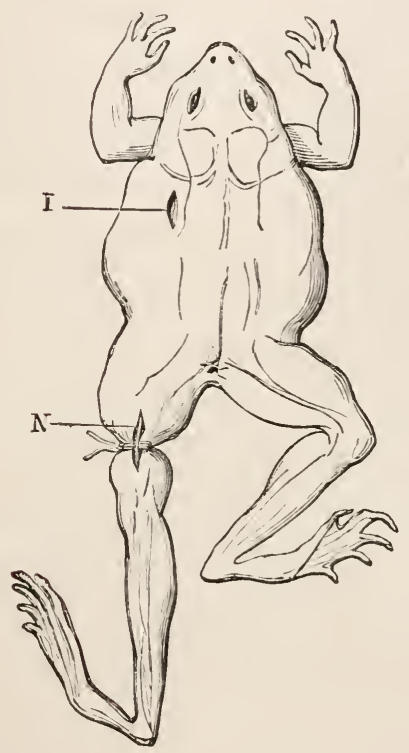

Fig. 11. Grenouille pour l'expérimentation 1 .

cette jambe pousse tout le reste du corps derant elle quand on place l'animal dans l'eau.

1. I, incision pour l'introduction du curare; $N$, nerf sciatique isolé. 
Quand, au lieu d'une jambe, on ne préserve de l'empoisonnement qu'un seul doigt, ce doigt s'agite et exprime le sentiment de tout le corps réduit à l'état de cadavre.

Le spectacle intéressant que je viens de tracer peut s'observer parfois pendant une heure ou deux dans les saisons favorables. Il ne cesse que lorsque l'asphyxie et la mort de l'organisme sont arrivées par suite de la suppression trop prolongée des mouvements respiratoires.

Chez les animaux à sang chaud, ces phénomènes se passent en un temps beaucoup plus court, mais ils n'en existent pas moins.

Lorsqu'un mammifère ou un homme est empoisonné par le curare, l'intelligence, la sensibilité et la volonté ne sont point atteintes par le poison, mais elles perdent successivement les instruments du mouvement, qui refusent de leur obéir. Les mouvements les plus expressifs de nos facultés disparaissent les premiers, d'abord la voix et la parole, ensuite les mouvements des membres, ceux de la face et du thorax, et enfin les mouvements des yeux qui, comme chez les mourants, persistentles derniers. 
Peut-on concevoir une souffrance plus horrible que celle d'une intelligence assistant ainsi à la soustraction successive de tous les organes qui, suivant l'expression de M. de Bonald, sont destinés à la servir, et se trouvant en quelque sorte enfermée toute vive dans un cadavre? Dans tous les temps, les fictions poétiques qui ont voulu émouvoir notre pitié nous ont représenté des êtres sensibles renfermés dans des corps immobiles. Notre imagination ne saurait rien concevoir de plus malheureux que des êtres pourvus de sensation, c'est-à-dire pouvant éprouver le plaisir et la peine, quand ils sont privés du pouvoir de fuir l'un et de tendre vers l'autre. Le supplice que l'imagination des poëtes a inventé se trouve produit dans la nature par l'action du poison américain. Nous pouvons même ajouter que la fiction est restée ici audessous de la réalité. Quand le Tasse nous dépeint Clorinde incorporée vivante dans un majestueux cyprès, au moins lui a-t-il laissé des pleurs et des sanglots pour se plaindre et attendrir ceux qui la font souffrir en blessant sa sensible écorce. 
Le poison est donc, ainsi que nous l'avons dit en commencant cette étude, un instrument qui nous a fait pénétrer dans les replis les plus cachés de notre organisation, et nous a permis d'en saisir les phénomènes les plus délicats. En parcourant les diverses phases de l'empoisonnement, nous avons vu que le curare détruit le mouvement en laissant persister la sensibilité. De plus, nous avons prouvé qu'il n'atteint qu'un des éléments efficaces du mouvement, le nerf moteur, car le cœur continue à battre, et les muscles ont conservé leur faculté contractile intacte.

La conclusion physiologique qui ressort de ces expériences est très-claire : l'élément nerveux sensitif, l'élément nerveux moteur et l'élément musculaire ont chacun leur autonomie, puisque le curare les sépare et n'est toxique que pour un seul d'entre eux. Rappelons-nous pourtant que, malgré leur indépendance; les éléments organiques n'ont d'effet physiologique que par l'ensemble de leurs rapports. La manifestation motrice chez l'homme ou chez un animal exige le concours de trois termes ou élé- 
ments anatomiques. L'élément nerveux, sensitif ou volontaire est le point de départ de la détermination motrice. Ensuite l'élément nerveux moteur transmet celte détermination au muscle qui l'exécute, ou autrement dit qui la manifeste. Si un seul des trois termes précédents vient à manquer, l'acte n'a plus lieu. Dans l'empoisonnement par le curare, la sensibilité ainsi que la volonté du mouvement existent, la contractilité et par conséquent la possibilité d'exécution du mouvement existent; mais par cela seul que l'élément nerveux moteur qui forme le trait d'union de la sensibilıté au mouvement est détruit par le poison, tout nous semble anéanti. En effet, la sensibilité, comme toutes les facultés qui ont pour siége le système nerveux, n'a aucune possibilité de se manifester par ellemême. Il faut absolument à ces facultés le système contractile ou musculaire sous une forme quelconque pour signaler leur présence ou se traduire à l'extérieur. Par conséquent nous ne pouvons juger des sensations des hommes et des animaux que par leurs mouvements. Cependant, chez les animaux empoisonnés par le 
curare, nous aurions été dans l'erreur la plus complète, si de l'absence du mouvement nous avions conclu à l'absence de la sensibilité. Cet exemple prouvera une fois de plus que nous n'avons de criterium absolu que dans notre conscience, et que dès que nous nous livrons aux interprétations des phénomènes qui sont en dehors de nous, nous ne sommes entourés que de causes d'erreur et d'illusions.

\section{V}

La science s'arrête aux causes prochaines des phénomènes; la recherche des causes premières n'est pas de son domaine. Le savant a donc atteint son but quand, par une analyse expérimentale successive, il est parvenu à rattacher la manifestation des phénomènes à des conditions matérielles exactement définies. De cause en cause il arrive finalement, suivant l'expres- 
sion de Bacon, à une cause sourde qui n'entend plus nos questions et ne répond plus. Toutefois la cause prochaine à laquelle nous devons nous arrêter ne peut jamais être considérée comme la limite absolue de nos connaissances; elle n'est sourde qu'à nos trop faibles moyens actuels d'investigation.

Dans notre analyse physiologique, nous sommes arrivés à localiser l'action du poison américain sur l'élément nerveux moteur et déterminer, comme conséquence, un mécanisme de la mort propre à cet agent toxique; mais devons-nous nous arrêter là et sommes nous parvenus à la limite que la science actuelle nous permet d'atteindre? Je ne le pense pas. Nonseulement il y aurait encore lieu d'isoler chimiquement le principe actif du curare des matières étrangères auxquelles il est mélangé; il y aurait en outre à déterminer quel genre de modification physique ou chimique la substance toxique imprime à l'élément organique pour en paralyser l'action. Quant à présent, nous ignorons complétement quelle peut être la nature de cette influence. Cependant nous savons à 
ce sujei une chose importante, c'est que, loin de produire une altération toxique définitive qui détruise pour toujours l'élément organique, ainsi que le font beaucoup de poisons, le curare ne détermine qu'une sorte d'inertie ou d'engourdissement de l'élément nerveux moteur. Il en résulte une paralysie de cet élément qui dure tant que le curare reste dans le sang en contact arec lui, mais qui peut cesser quand le poison est éliminé. De là il résulte cette conséquence inıportante, que la nıort par le curare n'est point sans appel, et qu'il est possible de faire revenir à la vie un animal ou un homme qui aurait été empoisonné par cet agent toxique.

Pour comprendre le mécanisme du retour à la vie, il faut nous rappeler le mécanisme de la mort, et si la théorie que nous en avons donnée est bonne, les deux mécanismes doivent se contrôler réciproquement et pouvoir se déduire l'un de l'autre.

Le curare introduit avec le sang va se mettre en contact avec les éléments organiques et paralyser d'une manière successive tous les 
mouvements volontaires. D'abord les nerf's moteurs des organes de la voix sont paralysés; mais la vie n'en continue pas moins, parce que l'animal respire toujours. Ce n'est que quand les mouvements respiratoires du thorax viennent à cesser que la mort réelle de l'organisme commence. Tous les éléments organiques du corps vont alors être atteints, parce qu'un élément indispensable à tous, l'air ou l'oxygène, va manquer dans le sang, leur milieu organique. Sans doute le cœur, qui continue à battre, fait circuler le sang, mais ce sang ne prend plus d'oxygène dans les poumons paralysés, et l'asphyxie de tous les éléments organiques arrivera avec une rapidité plus ou moins grande suivant la nature des animaux, mais d'une manière infaillible pour tous. Nous royons ainsi que la destruction de l'élément nerveux moteur ne tue pas directement, comme si cet élément seul représentait le principe de la vie. La soustraction de l'élément nerveux moteur tue parce que, les autres éléments qui avaient des rapports avec lui ne pouvant plus fonctionner, il en résulte une dislocation de la machine vivante 
tout entière. De même un édifice s'écroule quand on enlève une de ses pierres fondamentales.

En résumé, c'est donc le manque d'oxygène ou l'asphyxie qui amène la mort dans l'empoisonnement par le curare. S'il en est ainsi, c'est l'oxygène qu'il faut rendre pour rappeler à la vie, et le contre-poison sera simplement la respiration artificielle, c'est-à-dire un soufflet qui, remplacant les mouvements respiratoires éteints, introduira graduellement, et avec les précautions convenables, de l'air pur dans les poumons. On peut dire alors qu'on tient dans ses mains l'existence de l'individu empoisonné, et la vie nous apparaît comme un pur mécanisme dont nous pouvons faire mouvoir les rouages, mais que nous ne pouvons localiser dans aucun d'eux exclusivement; elle n'est nulle part et se rencontre partout.

Sous l'influence de la respiration artificielle, le sang continuera done à circuler et à se charger d'oxygène : de cette manière, les éléments organiques que le curare n'a pas atteints continueront à vivre; mais le poison lui-même, en circulant avec le sang, finira par s'éliminer 
par les divers émonctoires et particulièrement par les urines, de sorte qu'après un temps suffisant tout le curare sera sorti du sang, et l'élément nerveux moteur, qui n'avait été qu'engourdi par son contact, mais non désorganisé, se réveillera en quelque sorte et reprendra ses fonctions dès que l'agent qui le paralysait aura disparu. Alors le ronage vital brisé sera raccommodé, et la machine pourra reprendre et entretenir seule son mouvement naturel.

Telle est l'explication très-simple du retour à la vie des animaux empoisonnés par le curare au moyen de la respiration artificielle.

En 1815, Watterton et Brodie inoculèrent du curare à une ânesse, qui mourut en dix minutes. On lui fit alors une incision à la trachée artère, et on lui gonfla régulièrement les poumons pendant deux heures avec un soufflet. La vie suspendue revint : l'ânesse leva la tête et regarda autour d'elle; mais, l'introduction de l'air ayant été interrompue, elle retomba dans la mort apparente. On recommenca aussitôt la respiration artificielle et on la continua sans interruption pendant deux heures encore. 
Ce moyen sauva l'ânesse; elle se leva et marcha sans paraître éprouver ni agitation ni douleur. La blessure du cou et celle par laquelle le poison était entré guérirent facilement. Après un peu de fatigue, l'animal se rétablit tout à fait et devint par la suite gras et pétulant.

D'autres expérimentateurs, M. Virchow de Berlin entre autres, ont observé des faits semblables sur des chiens, des chats et des lapins.

J'ai souvent moi-même répété ces expériences et constaté que chez l'animal sauvé le poison était passé dans l'urine, de sorte qu'en concentrant ce liquide, on y retrouvait le curare avec ses propriétés toxiques ordinaires.

L'insufflation artificielle peut très-bien être appliquée à l'homme, et il existe des appareils pour la pratiquer.

Si un homme était empoisonné par lecurare, la seule manière connue de le sauver consisterait à le faire respirer artificiellement.

Mais, quand on peut agir aussitôt après la blessure, il y a d'autres moyens d'empêcher l'empoisonnement d'avoir lieu, non par des médications empiriques et illusoires, mais par 
des procédés physiologiques dont la science comprend et règle l'action. Si la blessure a eu lieu dans un membre, la première chose à faire est de poser une ligature sur ce membre audessus de la plaie empoisonnée. Nous savons qu'en empêchant ainsi le curare d'arriver au cœur, on s'oppose à l'empoisonnement de l'organisme; mais que faire ensuite? Le poison est toujours là, et si l'on enlève le bandage, l'intoxication, que l'on a retardée ou suspendue, n'en arrivera pas moins. Il n'yaurait à prendre qu'un parti extrême, qui du reste a été conseillé : à l'aide d'un couteau, enlever toute la surface empoisonnée ou, pour plus de sûreté encore, retrancher le membreau-dessous de la ligature. Sans doute, l'amputation serait préférable à une mort certaine; mais on peut mieux faire, car si nous réfléchissons aux notions expérimentales que nous avons acquises, nous verrons que la physiologie nous fournit la possibilité d'éviter à la fois la mort et la perte du membre.

Rappelons-nous qu'un animal empoisonné par le curare n'est pas privé de tous ses mouve- 
ments à la fois : on les voit s'éteindre successivement, en commencant par les mouvements des extrémités et en finissant par les mouvements respiratoires. Cet envahissement progressif de l'appareil locomoteur provient de l'action d'une dose graduellement croissante de poison introduite dans le sang par l'absorption, car lorsqu'on injecte d'un seul coup dans la circulation une forte proportion de curare, l'animal est comme foudroyé et meurt instantanément. Ceci nous prouve en outre qu'il y a des éléments nerveux moteurs qui sont plus accessibles à l'action du curare que d'autres. En effet, bien qu'il s'agisse d'éléments organiques de même nature, il y a entre eux une hiérarchie physiologique, de même qu'il y a une classification zoologique qui exprime la hiérarchie des organismes. La quantité de curare arrivée dans le sang et capable d'empoisonner les nerfs moteurs des membres ne suffit pas pour agir sur les nerfs moteurs de la tête : la quantité qui paralyse les nerfs moteurs de la tête n'atteint pas encore les nerfs respiratoires thoraciques et diaphragmatiques, mais d'un autre côté cette 
différence dans la susceptibilité des éléments pour le poison coïncide avec une vibration moins rapide de leur substance, de telle sorte que ceux qui sont les plus longs à s'empoisonner sont en même temps les plus tardifs à se débarrasser de la substance toxique. Les nerfs moteurs des membres et de la tête, qui sont empoisonnés avant les nerfs respiratoires, reprennent leurs fonctions avant ces derniers. C'est ce qui nous explique comment l'ânesse de Watterton, qui a pu relever la tête et regarder autour d'elle, est retombée morte quand on a arrêté le soufflel qui la faisait vivre en remplacant ses nerfs respiratoires encore engourdis.

De cet ensemble d'observations il résulte que nous pouvons, en variant les doses lu curare, passer en quelque sorte du poison au médicament, empoisonner l'animal complétement ou incomplétement, et même l'empoisonner au tiers, au quart, etc., de manière à obtenir des effets qui non-seulement ne soient pas mortels, mais qui soient gradués et déterminés d'avance.

J'ai institué depuis longtemps un grand nombre d'expériences de ce genre : j'ai pu ainsi 
amener des animaux à avoir seulement les quatre membres paralysés, ou bien les quatre membres et la tête. Enfin jai pu aller plus loin et paralyser les mouvements thoraciques en ne conservant intègre que le nerf diaphragmatique, qui suffit pour empêcher l'asphyxie.

Le curare sert ainsi de moyen contentif au physiologiste, car les animaux, exactement comme s’ils étaient solidement attachés sur une table de laboratoire (fig. 12 et 13 ', sont véritablement enchaînés pendant plusieurs heures dans de telles expériences, qui offrent d'ailleurs de l'intérêt à beaucoup d'autres points de vue. On observe alors, quand le curare agit en petite proportion, des sortes d'agitation non douloureuses dans les membres, par suite de cette loi que toute substance qui, à haute dose, éteint les propriétés d'un élément organique, les excite à petite dose. Quand l'action du curare est arrivée à son summum, l'élimination fait peu à peu disparaître le poison du sang; en même temps et parallèlement cessent tous les symptômes paralytiques; puis, aussitôt qu'ils sont dissipés, l'animal se lève et court alerte absolument 
comme avant, et sans qu'il en résulte jamais aucun inconvénient ultérieur pour sa santé.

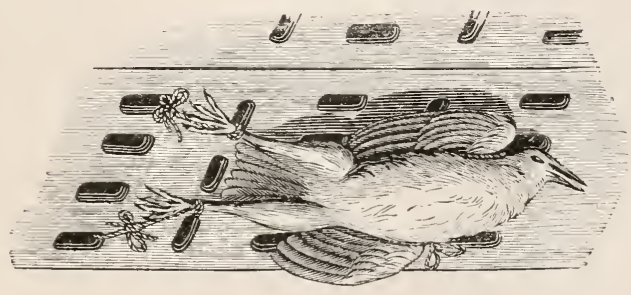

Fig. 12. Poulet sur la table du laboratoire.

Revenons maintenant à notre blessé, dont il s'agit de sauver la vie et de conserver le membre.

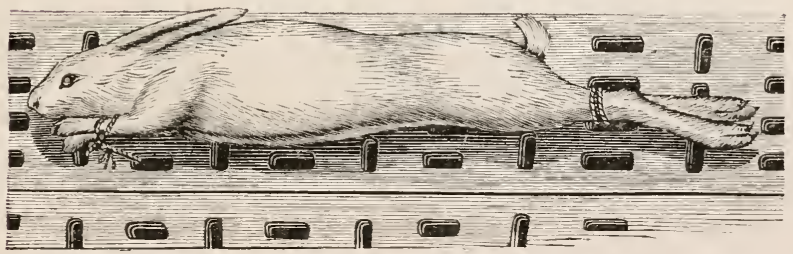

Fig. 13. Lapin sur la table du laboratoire.

La ligature est en place, et le poison est retenu au-dessous d'elle. On devine ce qu'il faut 
faire : délier le bandage et laisser pénétrer le poison dans le sang; mais dès que les membres seront pris et que la paralysie se manifestera, resserrer aussitôt la ligature; puis, quand l'élimination aura chassé le poison et fait disparaître les effets toxiques, défaire aussitôt le bandage et laisser entrer une quantité non mortelle qui sera chassée à son tour, el ainsi de suite, jusqu'à élimination complète. Cela n'est point aussi long qu'on pourrait le penser, et en moins d'une demi-journée j'ai pu sauver des chiens de moyenne taille qui avaient été piqués avec une flèche empoisonnée.

Quand on place une ligature sur un membre pour arrêter le poison, il n'est pas nécessaire de serrer le lien outre mesure, ce qui pourrait amener l'engorgement et même la gangrène du membre; il suffit de comprimer modérément pour empêcher le retour du sang veineux. On peut même dire qu'on n'arrête pas d'une manière absolue le passage diu sang empoisonné; mais il s'en échappe si peu à la fois que la petite quantité de poison introduite dans l'organisme est éliminée à mesure, sans pouvoir s'uc- 
cumuler assez pour produire ses effets toxiques. Cela explique comment j'ai pu empêcher des animaux d'être empoisonnés en laissant la ligature appliquée pendant vingt-quatre ou quarantehuit heures. Après ce temps on peut délier le membre sans danger, parce que le poison et la mort ont pu s'enfuir d'une manière imperceptible.

Le poison américain dont nous venons d'esquisser l'histoire physiologique est destiné, comme tous les poisons violents, à entrer dans la classe des remèdes héroïques; mais l'action thérapeutique des poisons, qui est encore aujourd'hui à peu près complétement dans les mains de l'empirisme, ne pourra en sortir et être compris scientifiquement que par l'étude physiologique des empoisonnements. L'action médicamenteuse n'est au fond qu'un empoisonnement incomplet.

C'est aux éléments intimes de notre organisation qu'il faut remonter pour saisir le mécanisme de toutes ces actions. Ces recherches sont longues et entourées de difficultés innombrables; mais les phénomènes de la vie ont leur déterCLAUDE BERNARD. 
minisme absolu, comme tous les phénomènes naturels.

La science vitale existe, elle n’a d'entraves que dans sa complexité, et s'il arrive un jour, ce qui n'est pas douteux, qu'à force de travail et de patience la physiologie soit définitivement fondée comme science, alors nous pourrons, par des modifications du milieu sanguin, exercer notre empire sur tout ce monde d'organismes élémentaires qui constituent notre être; en connaissant les lois qui régissent leurs rapports divers, nous pourrons régler et modifier à notre gré les manifestations vitales.

Sans doute le principe des choses nous échappera toujours, et nous ne cherchons pas à connaître l'origine première de tous ces éléments organiques, pas plus que le physicien et le chimiste ne cherchent à trouver la cause créatrice de la matière minérale dont ils étudient les propriétés. Seulement nous connaîtrons la loi des phénomènes de la substance vivante et $\mathrm{cr}^{\mathrm{r}-}$ ganisée, et en nous soumettant à ces lois nous pourrons faire varier les actions qui en dépendent. Les physiciens et les chimistes n'agissent 
pas autrement quand ils gouvernent les phénomènes des corps bruts. C'est par métaphore qu'ils se disent les maîtres de la nature, car ils savent parfaitement bien qu'ils ne font qu'obéir à ses lois.

1er septembre 1864. 


\section{ÉTUDE}

\section{LA PHYSIOLOGIE DU CGEUR}

Pour le physiologiste, le cœur est l'organe central de la circulation du sang, et à ce titre c'est un organe essentiel à la vie; mais par un privilége singulier, qui ne s'est vu pour aucun autre appareil organique, le mot cœur est passé, comme les idées que l'on s'est faites de ses fonctions, dans le langage du physiologiste, dans le langage du poëte, du romancier et de l'homme du monde, avec des acceptions fort différentes. Le cœur ne serait pas seulement un moteur vital qui pousse le liquide sanguin dans toutes les parties de notre corps qu'il anime; 
le cœur serait aussi le siége et l'emblème des sentiments les plus nobles et les plus tendres de notre âme. L'étude du cœur humain ne serait pas uniquement le partage de l'anatomiste et du physiologiste; cette étude devrait aussi servir de base à toutes les conceptions du philosophe, à toutes les inspirations du poëte et de l'artiste.

Il s'agira ici, bien entendu, du cœur anatomique, c'est-à-dire du cœur étudié au point de vue de la science physiologique purement expérimentale; mais cette étude rapide que nous allons faire des fonctions du cœur devra-t-elle renverser les idées généralement reçues? La physiologie devra-t-elle nous enlever des illusions, et nous montrer que le rôle sentimental que dans tous les temps on a attribué au cœur n'est qu'une fiction purement arbitraire ? En un mot, aurons-nous à signaler une contradiction complète et péremptoire entre la science et l'art, entre le sentiment et la raison?...

Je ne crois pas, quant à moi, à la possibilité de cette contradiction. La vérité ne saurait différer d'elle-même, et la vérité du savant ne sau- 
rait contredire la vérité de l'artiste. Je crois au contraire que la science qui coule de source pure deviendra lumineuse pour tous, et que partout la science et l'art doivent se donner la main en s'interprétant et en s'expliquant l'un par l'autre. Je pense enfin que, dans leurs régions élevées, les connaissances humaines forment une atmosphère commune à toutes les intelligences cultivées, dans laquelle l'homme du monde, l'artiste et le savant doivent nécessairement se rencontrer et se comprendre.

Dans ce qui va suivre, je ne chercherai donc pas à nier systématiquement au nom de la science tout ce que l'on a pu dire au nom de l'art sur le cœur comme organe destiné à exprimer nos sentiments et nos affections.

Je désirerais au contraire, si j'ose ainsi dire, pouvoir affirmer l'art par la science en essayant d'expliquer par la physiologie ce qui n'a été jusqu'à présent qu'une simple intuition de l'esprit. Je forme, je le sais, une entreprise trèsdifficile, peut-être même téméraire, à cause de l'état actuel encore si peu avancé de la science des phénomènes de la vie. Cependant la beauté 
de la question et les lueurs que la physiologie me semble déjà pouvoir y jeter, tout cela me détermine et m'encourage. Il ne s'agira pas d'ailleurs de parler ici de la physiologie du cœur en entrant dans tous les détails d'une étude analytique expérimentale complète et impossible pour le moment : c'est une simple tentative, et il me suffira d'exprimer mes idées physiologiques en les appuyant par les faits les plus clairs et les plus précis de la science. J'envisagerai ainsi la physiologie du cœur d'une manière générale, mais en m'attachant plus particulièrement aux points qui me semblent propres à éclairer la physiologie du cœur de l'homme.

\section{I}

Avant tout, le cœur est une machine motrice vivante, une véritable pompe foulante destinée à distribuer le fluide nourricier et excitateur des 
fonctions à tous les organes de notre corps. Ce rôle mécanique caractérise le cœur d'une manière absolue, et partout où le cœur existe, quel que soit le degré de simplicité ou de complication qu'il présente dans la série animale, il accomplit constamment et nécessairement cette fonction d'irrigateur organique.

Pour un anatomiste pur, le cœur de l'homme est un viscère, c'est-à-dire un des organes qui font partie des appareils de nutrition situés dans les cavités splanchniques. Tout le monde sait que le cœur (fig. 14) est placé dans la poitrine, entre les deux poumons, qu'il a la forme d'un cône dont la base est fixée par de gros vaisseaux qui charrient le liquide sanguin, et dont la pointe libre est inclinée en bas et à gauche, de facon à venir se placer entre la cinquième et la sixième côte au-dessous du sein gauche. Quant à la nature du tissu qui le compose, le cœur rentre dans le système musculaire: il est creusé à l'intérieur de cavités qui servent de réservoir au sang; c'est pourquoi les anatomistes ont encore appelé le cœur un muscle creux.

Dans le cœur de l'homme, on voit quatre 
compartiments ou cavités : deux cavités for-

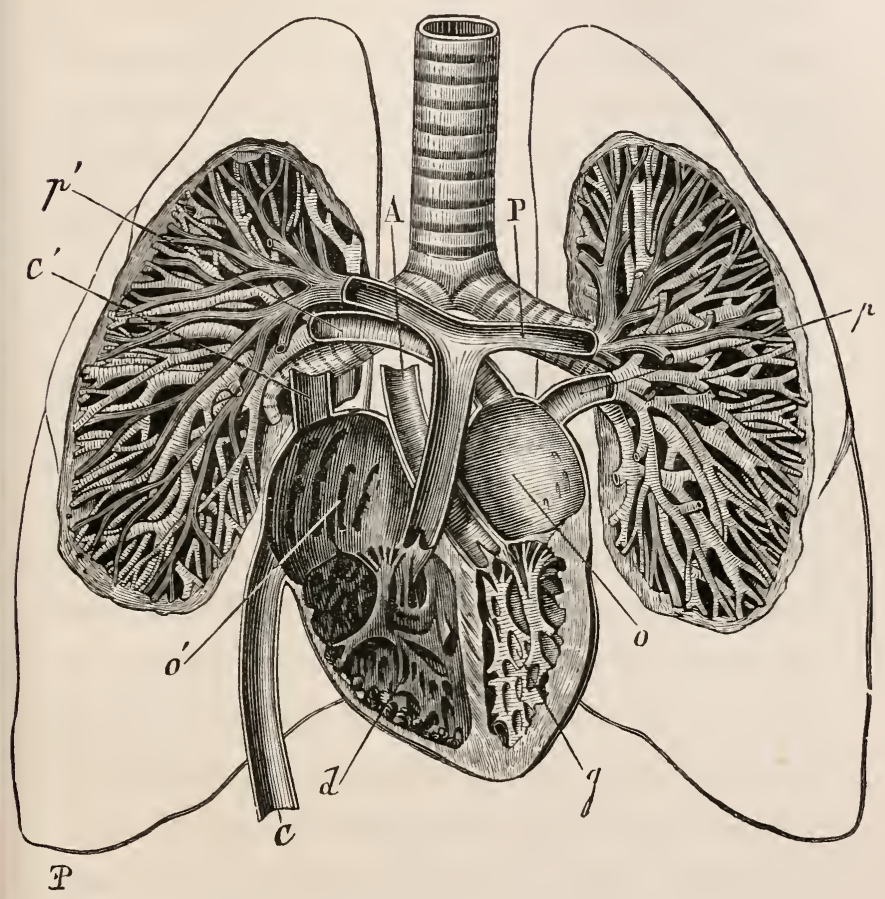

Fig. 14. Circulation du sang dans le cœur et dans le poumon '.

1. A, artère aorte sortant du cœur gauche; $\mathrm{P}$, artère pulmonaire partant du ventricule droit; $c, c^{\prime}$, veines caves inférieure et supérieure se rendant dans l'oreillette droite; $p, p^{\prime}$, veines pulmonaires droite et gauche se rendant dans l'oreillette gauche; $o$, oreillette gauche; $o^{\prime}$, oreillette droite; $d$, ventricule droit; $g$, ventricule gauche. 
ment la partie supérieure ou base du cœur, appelées oreillettes et recevant le sang de toutes les parties du corps au moyen de gros tuyaux nommés veines; deux cavités forment la partie inférieure ou la pointe du cœur, appelées ventricules et destinées à chasser le liquide sanguin dans toutes les parties du corps au moyen de gros tuyaux nommés artères.

Chaque oreillette du cœur communique avec le ventricule qui est au-dessous d'elle du même côté; mais une cloison longitudinale sépare latéralement les oreillettes et les ventricules, de telle sorte que le cœur de l'homme, qui est réellement double, se décompose en deux cœurs simples formés chacun d'une oreillette et d'un ventricule, et situés l'un à droite, l'autre à gauche de la cloison médiane.

Chaque cavité ventriculaire du cœur est munie de deux soupapes appelées valvules. L'une placée à l'orifice d'entrée du sang de l'oreillette dans le ventricule, est nommée valvule auriculoventriculaire; l'autre, située à l'orifice de sortie du sang du ventricule par l'artère, s'appelle valvule sigmoïde. 
Le cœur de l'homme, ainsi que celui des mammifères et des oiseaux, est donc un cœur anatomiquement double et composé de deux cœurs simples, appelés l'un le cœur droit, l'autre le cour gauche. Chacun de ces cœurs a un rôle bien différent. Le cœur gauche, nommé encore cœur à sang rouge, èst destiné à recevoir dans son oreillette par les veines pulmonaires le sang pur et rutilant qui vient des poumons, pour le faire passer ensuite dans son ventricule, qui le lance dans toutes les parties du corps, où il devient impur et noir. Le cœur droit, appelé aussi cœur à sang noir, est destiné à recevoir dans son oreillette par les veines caves le sangr impur qui revient de toutes les parties du corps et à le faire passer ensuite dans son ventricule pour le lancer dans le poumon, où il devient pur et rutilant. En un mot, le cœur gauche est le cœur qui préside à la distribution du liquide vital dans tous nos organes et dans tous nos tissus, et le cœur droit est lecœur qui préside à la révivification du sang dans les poumons, pour le restituer au cœur gauche, et ainsi de suite (fig. 15). 


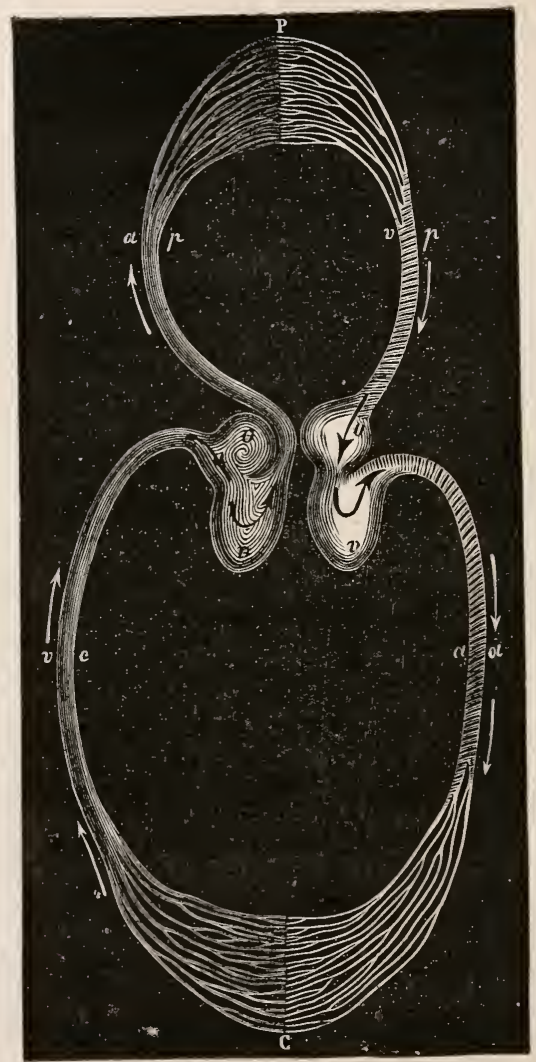

Fig. 15. Appareil de la grande et de la petite circulations.

1. 00, oreillettes; $v v$, ventricules; $a d$, système aortique; e, capillaires gẻnéraux; ve, veines à sang noir; ap, artère pulmonaire; $p$, capillaire du poumon; $v p$, veines à sang rouge. 
Ces prémisses étant établies, nous n'aurons plus ici à considérer le cœur que comme un organe qui distribue la vie à toutes les parties de notre corps, parce qu'il leur envoie le liquide nourricier qui leur est indispensable pour vivre et manifester leurs fonctions.

Quant au liquide nourricier, il est représenté par le sang lui-même, qui est sensiblement identique chez tous les animaux vertébrés quelles que soient d'ailleurs la diversité de l'espèce animale et la variété de son alimentation. Dans les phénomènes extérieurs de la préhension des aliments, le zoologiste distingue le carnassier féroce qui se nourrit de chairs palpitantes, le ruminant paisible qui se repaît de l'herbe des prés, le frugivore et le granivore qui se nourrissent plus spécialement de fruits et de graines; mais, quand on descend dans le phénomène intime de la nutrition, la physiologie générale nous apprend que ce qui se nourrit, à proprement parler, dans les animaux, ce n'est pas le type spécifique et individuel, qui varie à l'infini, mais seulement les organesélémentaires et les tissus, qui partout se détruisent et vivent CLAUDE BERNARD. 
d'une manière identique. La nature, suivant l'expression de Gœthe, est un grand artiste. Les animaux sont constitués par des matériaux organiques semblables; c'est l'arrangement et la disposition relative des matériaux qui déterminent la variété de ces véritables monuments organisés, c'est-à-dire les formes et les propriétés animales spécifiques. De même, dans les monuments de l'homme, les matériaux se ressemblent par leurs propriétés physiques, et cependant l'arrangement différent peut réaliser des idées diverses et donner naissance à un palais ou à une chaumière. En un mot, le type spécifique existe, mais seulement à l'état d'une idée réalisée. Pour la physiologie, ce n'est pas le type animal qui vit et meurt, ce sont les matériaux organiques ou les tissus qui le composent; de même, dans un édifice qui se dégrade, ce n'est pas le type idéal du monument qui se détériore, mais seulement les pierres qui le forment.

En physiologie générale, on ne saurait donc déduire de la grande variété d'alimentation des animaux aucune différence de nutrition orga- 
nique essentielle. Chez l'homme et chez tous les animaux, les organes élémentaires et les tissus vivants sont sanguinaires, c'est-à-dire qu'ils se repaissent du sang dans lequel ils sont plongés. Ils y vivent comme les animaux aquatiques dans l'eau, et de même qu'il faut renouveler l'eau qui s'altère et perd ses éléments nutritifs, de même il faut renouveler, au moyen de la circulation, le sang qui perd son oxygène et se charge d'acide carbonique. Or c'est précisément là le rôle qui incombe au cœur. Le système du cœur gauche apporte aux organes le sang qui les anime; le système du cœur droit emporte le sang qui les a fait vivre un instant.

Quand en physiologie on veut comprendre les fonctions d'un organe, il faut toujours remonter aux propriétés vitales de la substance qui le compose; c'est par conséquent dans les propriétés du tissu du cœur que nous pourrons trouver l'explication de ses fonctions. Cela ne nous offrira d'ailleurs aucune difficulté, car, ainsi que nous l'avons déjà dit, le cœur est un muscle, et il en possède toutes les propriétés 
physiologiques. Or il me suffira de rappeler que ce tissu charnu ou musculaire est constitué par des fibres qui ont la propriété de se raccourcir, c'est-à-dire de se contracter.

Quand les fibres musculaires sont disposées de manière à former un muscle allongé dont les deux extrémités viennent s'insérer sur deux os articulés ensemble, l'effet nécessaire de la contraction ou du raccourcissement du muscle est de faire mouvoir les deux os l'un sur l'autre en les rapprochant.

Mais quand les fibres musculaires sont disposées de manière à former les parois d'une poche musculaire, comme cela a lieu dans le cœur, l'effet nécessaire de la contraction du tissu musculaire est de rétrécir et de faire disparaitre plus ou moins complétement la cavité en expulsant le contenu. Cela nous fera comprendre comment, à chaque contraction des cavités du cœur, le sang qu'elles contiennent se trouve expulsé suivant une direction déterminée par la disposition des valvules ou soupapes cardiaques.

Quand l'oreillette se contracte, le sang est 
poussé dans le ventricule parce que la valvule auriculo-ventriculaire s'abaisse (fig. 16); quand le ventricule se contracte, le sang est chassé dans les artères parce que la valvule sygmoïde

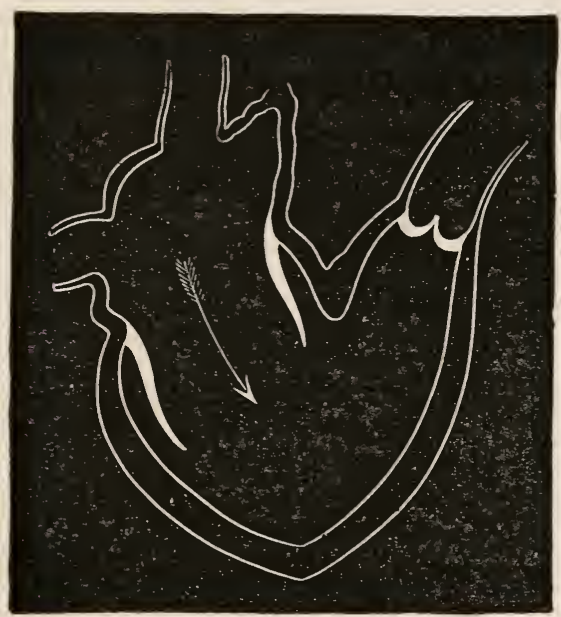

Fig. 16. Oreillette et ventricule droits '.

ou artérielle s'abaisse pour laisser passer le liquide sangain en même temps que la valvule auriculo-ventriculaire se relève pour empêcher

1. Valvules ventriculaires ouvertes; valvules semi-lunaires fermées. 
le sang de refluer dans l'oreillette (fig. 17). La contraction des cavités du cœur, qui les vide de sang, est suivie d'un relâchement pendant le-

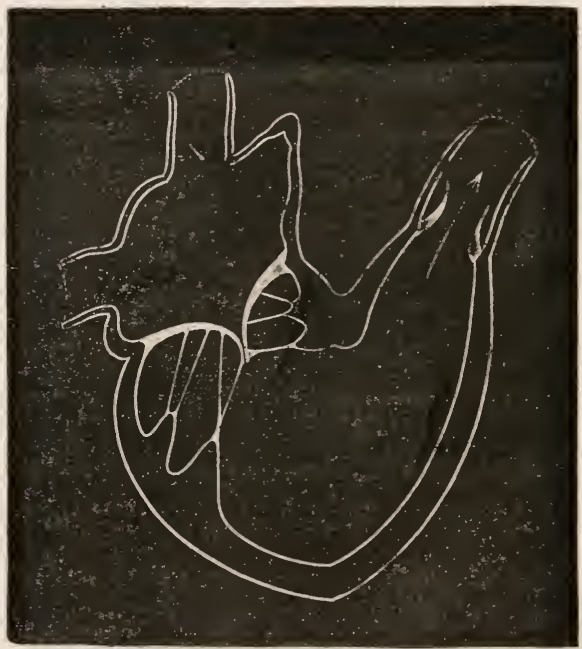

Fig. 17. Oreillette et ventricule droits ${ }^{1}$.

quel de nouveau elles se remplissent de liquide sanguin, puis d'une nouvelle contraction qui les vide encore, et ainsi de suite. Il en résulte

1. Valviules ventriculaires fermées; valvules semi-lunaires ouvertes. 
que le mouvement du cœur est constitué par une succession de mouvements alternatifs de contraction et de relâchement de ses cavités. On appelle systole le mouvement de contraction et diastole le mouvement de relâchement.

Les quatre cavités du cœur se contractent et se relâchent successivement deux à deux : d'abord les deux oreillettes, puis les deux ventricules. Un intervalle de repos très-court sépare la contraction des oreillettes de la contraction des ventricules, puis un intervalle un peu plus long succède à la contraction du ventricule.

Il serait complétement hors de notre objet de décrire ici en détail le mécanisme de la circulation dans les différentes cavités du cœur. Dans nos explicatons ultérieures, nous aurons seulement à tenir compte du ventricule gauche, qui, ainsi que nous l'avons déjà dit, est le ventricule nourricier qui alimente et anime tous les organes du corps. Il nous suffira donc de dire qu'au moment de la contraction de ce ventricule le cœur se projette en avant, et vient frapper comme le battant d'une cloche entre la cinquième et la sixième côte au-dessous du sein 
gauche; c'est ce qu'on appelle le battement $d u$ cœur. A ce même instant de la contraction du ventricule gauche, le sang est lancé dans l'aorte et dans les artères du corps arec une pression capable de soulever une colonne mercurielle d'environ 150 millimètres de hauteur. C'est ce qui produit le soulèvement observé dans toutes les artères, et qu'on appelle le pouls.

Toute la mécanique des mouvements du cœur a été l'objet de travaux extrêment approfondis, et la science moderne a étudié les phénomènes de la circulation à l'aide de procédés graphiques qui donnent aux recherches une très-grande exactitude.

Le seul point que nous tenions à rappeler, c'est que le cœur est une véritable machine vivante, qui fonctionne comme une pompe foulante dans laquelle le piston est remplacé par la contraction musculaire.

La question que nous désirons plus particulièrement examiner dans cette étude est celle de savoir comment le cœur, ce simple moteur de la circulation du sang, peut, en réagissant sous l'influence du système nerveux, coopérer 
au mécanisme si délicat des sentiments qui se passent en nous.

\section{II}

Le cœur nous apparaît immédiatement comme un organe étrange par son activité exceptionnelle.

Dans le développement du corps animal, chaque appareil vital n'entre en général en fonction qu'après avoir achevé son évolution et acquis sa texture définitive. Il y a même des organes, particulièrement ceux destinés à la propagation de l'espèce, qui ne se montrent sur la scène organique que longtemps après la naissance pour en disparaître ensuite et rentrer de nouveau dans la torpeur pendant la dernière période de la vie de l'individu.

Le cœur au contraire manifeste son activité dès l'origine de la vie, bien longtemps avant de posséder sa forme achevée et sa structure carac- 
téristique. Ce fait n'est pas seulement remarquable comme caractère de la précocité des fonctions du cœur, mais il est de nature à faire réfléchir profondément le physiologiste sur le rapport réel qui doit exister entre les formes anatomiques et les propriétés vitales des tissus.

Rien n'est beau comme d'assister à la naissance du cœur.

Chez le poulet (fig. 18), dès la vingt-sixième ou trentième heure de l'ineubation, on voit apparaître sur le champ germinal un très-petit point, punclum saliens, dans lequel on finit par constater des mouvements rares et à peine perceptibles.

Peu à peu ces mouvements se prononcent davantage et deviennent plus fréquents; le cœur se dessine mieux, des artères et des veines se forment, le liquide sanguin se manifeste plus distinctement, et tout un système vasculaire provisoire (area vasculosa) s'est étalé en rayonnant autour du cœur, désormais constitué physiologiquement comme organe de circulation embryonnaire. A ce moment, les linéaments fondamentaux du corps de l'animal ont déjà 
paru; le cœur, alors en pleine activité, représente un moteur sanguin isolé, antérieur à l'organisation, et destiné à transporter sur le chantier de la vie les matériaux nécessaires à la formation du corps animal. Chez l'oiseau, le cœur va chercher les matériaux dans les éléments de l'œuf : chez le mammifère, il les puise dans les éléments du sang maternel.

Pendant que cet organe sert ainsi à la construction et au développement du corps tout entier, il s'accroît et se développe lui-même. A son origine, ce n'est qu'une simple vésicule obscurément contractile, comme la vésicule circulatoire d'un infusoire; mais cette vésicule s'allonge bientôt et bat avec rapidité; la partie inférieure recoit le liquide sanguin et représente une oreillette, tandis que la partie supérieure constitue un véritable ventricule qui lance le sang dans un bulbe aortique se divisant en ares branchiaux : c'est alors un vrai cœur de poisson. Plus tard, ce cœur subit un mouvement combiné de torsion et de bascule qui ramène en haut sa partie auriculaire et en bas sa partie ventriculaire; avant que le mouve- 


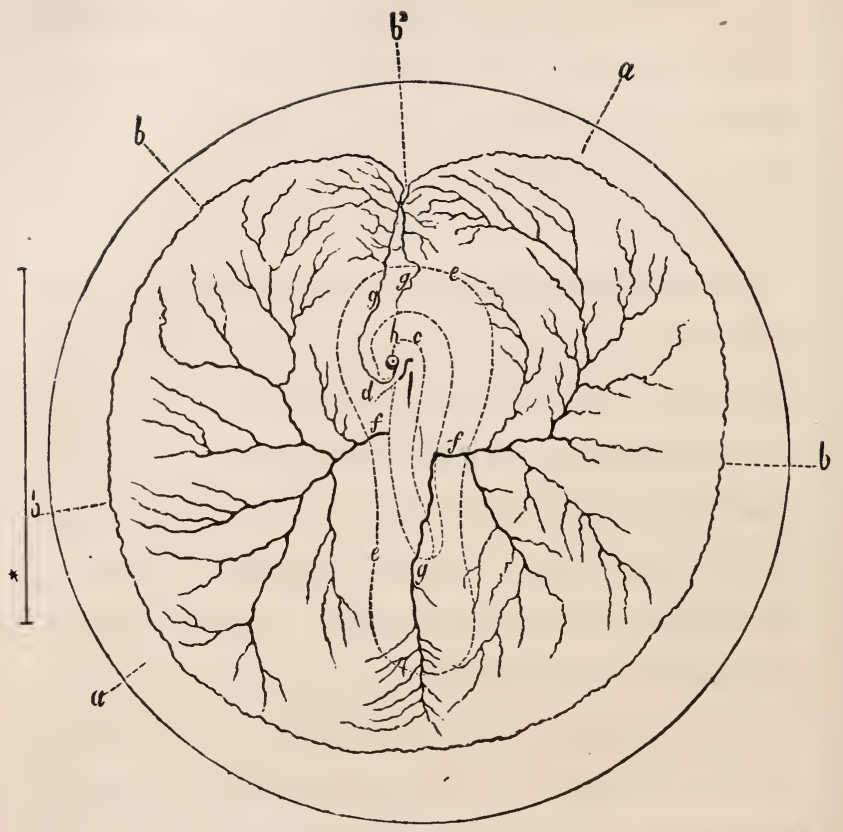

Fig. 18. Jaune d'œuf de poule '.

1. Plus que doublé de grandeur, pour faire voir la circulation du sang dans le blastoderme : $a$, jaune; $b$, sinus terminal; $b^{2}$, immersion supérieure du sinus terminal; $e$, aorte; $d$, points pulsatifs du cœur; $f f$, artères du blastoderme; 999 , veines du blastoderme (une inférieure et deux supérieures; celle-ci est parfois simple); ce, arca pellucida, en forme de biscuit; $h$, l'œil. On a omis les ramifications les plus déliées et $\mathrm{s}$ anastomoses avec le sinus terminal. (Wagner,) 
ment de bascule soit complet, l'organe représente un cœur à trois cavités, cœur de reptile, et dès que le mouvement est achevé, il possède les quatre cavités du cour d'oiseau ou de mammifère.

Les diverses phases de développement $\mathrm{du}$ cœur nous montrent donc que cet organe n'arrive à son état d'organisation le plus élevé chez les oiseaux, les mammifères et l'homme, qu'en passant transitoirement par des formes qui sont restées définitives pour des classes animales inférieures. C'est l'observation de ces faits et de beaucoup d'autres du mıême genre qui a donné naissance à l'idée philosophiquement vraie que chaque animal reflète dans son évolution embryonnaire les organismes qui lui sont inférieurs.

Le cœur diffère ainsi de tous les muscles du corps en ce qu'il agit dès qu'il apparaît, et avant d'être complétement développé.

Une fois achevé dans son organisation, il continue encore de former une exception dans le système musculaire : en effet, tous les appareils musculaires nous présentent dans leurs 
fonctions des alternatives d'activité et de repos; le cœur au contraire ne se repose jamais. De tous les organes du corps il est celui qui agit le plus longtemps; il préexiste à l'organisme, il lui survit, et dans la mort successive el naturelle des organes il est le dernier à manifester ses fonctions. En un mot, suivant l'expression du grand Haller, le cœur vit le premier (primum vivens) et meurt le dernier (ultimum moriens). Dans cette extinction de la vie de l'organisme, le cœur agit encore quand déjà les autres organes font silence auiour de lui. Il veille le dernier, comme sil attendait la fin de la lutte entre la vie et la mort, car tant qu'il se meut, la vie peut se rétablir; lorsque le cœur a cessé de battre, elle est irrévocablement perdue, et de même que son premier mouvement a été le signe certain de la vie, son dernier battement est le signe certain de la mort.

Les notions qui précèdent étaient nécessaires à donner, car elles nous aideront à mieux faire comprendre l'action du système nerveux sur le cœur.

Nous devons déjà pressentir que cet organe 
musculaire possède la propriété de se contracter sans l'intervention de l'influence nerveuse; il entre en fonction bien avant que le système nerveux ait donné signe devie. Il y a même plus, les nerfs peuvent être très-développés et constitués anatomiquement sans agir encore sur aucun des organes musculaires qui sont eux: mêmes déjà développés. En effet, j'ai constaté par des expériences directes que les extrémités nerveuses ne se soudent physiologiquement aux systèmes musculaires que dans les derniers temps de la vie embryonnaire. Lorsque, après la naissance, le système nerveux a pris son empire sur tous les organes musculaires du corps, le cœur se passe néanmoins de son influence pour accomplir ses fonctions de moteur circulatoire central. On paralyse les muscles des membres en coupant les nerfs qui les animent, on ne paralyse jamais les mouvements du cœur en divisant les nerfs qui se rendent dans son tissu; au contraire, ses.mouvements n'en deviennent que plus rapides. Les poisons qui détruisent les propriétés des nerfs moteurs abo. lissent les mouvements dans tous les organes 
musculaires du corps, tandis qu'ils sont sans action sur les battements du cœur. J'ai décrit ${ }^{1}$ les effets du curare, le poison paralyseur par excellence des systèmes nerveux moteurs; on se souvient que le cœur continue de battre et de faire circuler le sang dans le corps d'un animal absolument privé de toute influence nerveuse motrice.

De tout cela devons-nous conclure que le cœur ne possède pas de nerfs? Cette opinion, à laquelle s'étaient arrêtés d'anciens physiologistes, est aujourd'hui contredite par l'anatomie, qui nous montre que le cœur recoit dans son tissu un grand nombre de rameaux nerveux. Ce n'est donc pas à l'absence de nerfs qu'il faut attribuer toutes les anomalies que le cœur nous a offertes jusqu'à présent, c'est à l'existence d'un mécanisme nerveux tout particulier, qu'il nous reste à examiner.

1. Voy. le Curare, p. 237. 


\section{III}

La réaction bien connue des nerfs moteurs sur les muscles en général se résume en cette proposition fondamentale: tant que le nerf n'est point excité, le muscle reste à l'état de relâchement et de repos; dès que le nerf vient à être excité naturellement ou artificiellement, le muscle entre en activité et en contraction.

L'observation de l'influence de notre volonté sur les mouvements de nos membres suffirait pour nous prouver ce que je viens d'avancer; mais rien n'est en outre plus facile à démontrer par des expériences directes faites sur des animaux vivants ou récemment morts.

Si par vivisection on prépare une grenouille (fig. 19), de manière à isoler un nerf qui se rend dans les muscles d'un membre, on voit que, tant qu'on ne touche pas à ce nerf, les 


\section{musles du membre restent relâchés et en repos,}

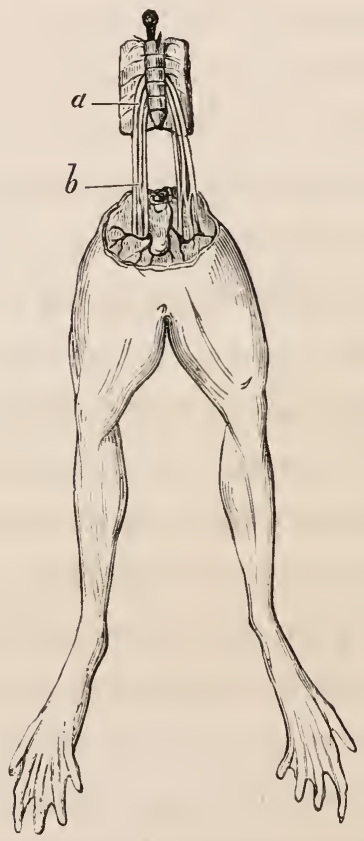

Fig. 19. Grenouille tuée par décapitation. Train postérieur '.

et qu'aussitôt qu'on vient à exciter ce nerf par

1. La colonne vertébrale est coupée de manière que le fragment supérieur $a$ serve à fixer un crochet et que sa séparation d'avec le fragment inférieur laisse un espace libre, dans lequel apparaissent les nerfs lombaires $b$. 
le pincement ou mieux par un courant électrique, les muscles entrent en une contraction énergique et rapide. C'est là un fait général qui peut se constater expérimentalement chez l'homme et chez tous les animaux vertébrés, soit pendant la vie, soit immédiatement après la mort, tant que les systèmes musculaires et nerveux conservent leurs propriétés vitales respectives.

Si maintenant nous agissons par des procédés analogues sur les nerfs du cœur, nous verrons que cet organe musculaire paradoxal nous présente encore à ce point de vue une exception, et je dirai même, pour être plus exact, qu'il nous offre une complète opposition avec les muscles des membres. Pour être dans la vérité, il suffira de renverser les termes de la proposition et de dire: Tant que les nerfs du cœur ne sont pas excités, le cœur bat et reste à l'état de fonction; dès que les nerfs du cœur viennent à être excités naturellement ou artificiellement, le cœur entre en relàchement et à l'état de repos. Si on prépare par vivisection une grenouille ou un autre animal vivant ou récemment mort de 
manière à observer le cœur et à isoler les nerfs pneumo-gastriques qui vont dans son tissu, on constate que, tant qu'on n'agit pas sur ces nerfs, le cœur continue à battre comme à l'ordinaire, et qu'aussitôt qu'on vient à les exciter par un courant électrique puissant, le cœur s'arrête en diastole, c'est-à-dire en relâchement.

Ce résultat est également général; il existe chez tous les vertébrés depuis la grenouille jusqu'à l'homme.

Il faudra toujours avoir présent à l'esprit le fait de cette influence singulière et paradoxale des nerfs sur le cœur, parce que c'est ce résultat qui nous servira de point de départ pour expliquer ultérieurement comme l'organe central de la circulation peut réagir sur nos sentiments; mais, avant d'en arriver là, il est nécessaire d'examiner de plus près les diverses formes que peut nous présenter l'arrêt du cœur sous l'influence de l'excitation galvanique des nerfs.

L'excitation des nerfs pneumo-gastriques ou nerfs du cœur par un courant électrique très- 
actif arrète aussitôt les battements de cet organe. Toutefois il y a dans le phénomène quelques variétés qui dépendent de la sensibilité de l'animal. Si l'on agit sur des mammifères très-sensibles, le cœur s'arrête instantarément, tandis que chez des animaux à sang froid et surtout pendant l'hiver le cœur ne ressent pas immédiatement l'influence nerveuse; plusieurs battements peuvent encore avoir lieu avant qu'il s'arrête. Après la cessation de l'excitation galvanique violente des nerfs, les battements reparaissent assez vite, plus ou moins facilement toutefois, suivant l'état de vigueur ou de sensibilité de l'animal. Il peut même arriver que chez des animaux très-sensibles ou affaiblis les battements ne reparaissent plus; alors l'arrêt du cœur est définitif, et la mort s'ensuit immédiatement.

L'excitation galvanique des nerfs pneumogastriques a pour effet d'arrêter le cœur d'autant plus énergiquement que l'application en estplus soudaine et qu'elle a été moins répétée. Quand on reproduit plusieurs fois de suite ou qu'on prolonge trop l'excitation, la sensibilité du cœur 
et de ses nerfs s'émousse au point que l'électricité ne peut plus arrêter ses battements; il en est de même quand on irrite graduellement les nerfs : on peut arriver successivement à employer des courants très-violents sans arrêter le cœur. Lorsqu'on applique des excitations faibles sur les nerfs du cœur, les résultats sont toujours les mêmes au fond, seulement la différence d'intensité leur donne une apparence tout autre. En effet, l'excitation galvanique faible et instantanée des pneumo-gastriques amène bien chez un animal très-sensible un arrêt subit du cœur, mais de si courte durée qu'il serait souvent imperceptible pour un observateur non prévenu. En outre, à la suite de ces actions légères ou modérées, les battements cardiaques reparaissent aussitôt avec plus d'énegie et de rapidité. On voit ainsi que l'excitation énergique des nerfs du cœur amène un arrêt prolongé del'organe, avec un retour lent et plus ou moins difficile de ses battements, tandis que les actions modérées ne provoquent qu'un arrêt extrêmement fugace du cœur, suivi immédiatement d'une accélération dans ses batte- 
ments avec augmentation de l'énergie des contractions ventriculaires.

Tous les résultats que nous avons mentionnés jusqu'ici, soil relativement à l'excitation des nerfs des muscles des membres, soit relativement à l'excitation des nerfs du cœur, ont été fournis par des expériences de vivisection dans lesquelles on avait appliqué l'excitant sur les nerfs moteurs eux-mêmes; mais dans l'état naturel les choses ne sauraient se passer ainsi : ce sont des excitants physiologiques qui viennent irriter les nerfs moteurs, afin de déterminer leur réaction sur les muscles. Ces excitants physiologiques sont au nombre de deux : la volonté et la sensibilité. La volonté ne peut exercer son influence sur tous les nerfs moteurs du corps; les nerfs du cœur par exemple sont en dehors d'elle. La sensibilité au contraire exerce une influence qui est générale, et tous les nerfs moteurs, qu'ils soient volontaires ou involontaires, subissent son action réflexe. On a appelé réflexes toutes les actions sensitives qui réagissent sur les nerfs moteurs en donnant lieu à des mouvements involontaires, parce 
qu'on suppose que l'impression sensitive venue de la périphérie est réfléchie dans le centre nerveux sur le nerf moteur.

Il serait inutile de nous étendre davantage sur le mécanisme des actions nerveuses réflexes, qui forment aujourd'hui une des bases importantes de la physiologie du système nerveux ${ }^{1}$. Il nous suffira de savoir que tous les mouvements involontaires sont le résultat de la simple action de la sensibilité ou du nerf sensitif sur le nerf moteur, qui réagit ensuite sur le muscle. Tous les mouvements involontaires $\mathrm{du}$ cœur que nous aurons à observer n'ont pas d'autre source que la réaction de la sensibilité sur les nerfs pneumo-gastriques moteurs de cet organe, et quand nous dirons par exemple qu'une impression douloureuse arrête les mouvements du cœur, cela signifiera simplement qu'un nerf sensitif primitivement excité a transmis son impression au cœur en excitant le pneumo-gastrique, qui, à son tour, a fait

1. Voyez Claude Bernard, Leçons sur la physiologie et la pathologie du système nerveux. Paris, 1858. 
ressentir son influence motrice au cœur absolument comme quand nous agissons dans nos expériences avec le courant galvanique. Quand le physiologiste excite un nerf moteur à réagir sur les muscles au moyen d'un courant galvanique ou à l'aide du pincement, il substitue un excitant artificiel à l'excitant naturel, qui est la volonté ou la sensibilité ; mais les résultats de l'action nerveuse motrice sont toujours les mêmes. On verra bientôt en effet toutes les formes d'arrêt du cour que nous avons observées en agissant directement avec un courant galvanique sur les nerfs pneumo-gastriques se reproduire par les influences sensitives diverses. Comme nous savons maintenant que les influences sensitives ne peuvent agir sur le cour qu'en excitant ses nerfs moteurs, nous sousentendrons désormais cet intermédiaire dans le langage, et quand nous dirons: la sensibilité ou les sentiments réagissent sur le cœur, nous saurons ce que cela signifie physiologiquement.

Nos expériences directes sur l'excitation des nerfs pneumo-gastriques nous ont montré que le cœur est d'autant plus prompt à recevoir 
l'impression nerveuse et à s'arrêter que l'animal est plus sensible; il en est de même pour les réactions des nerfs de la sensibilité sur le cœur.

Chez la grenouille, on n'arrête pas le cœur en pincant la peau : il faut des actions beaucoup plus énergiques.

Mais chez des animaux élevés, chez certaines races de chiens par exemple, les moindres excitations des nerfs sensitifs retentissent sur le cœur. Si l'on place un hémomètre sur l'artère de l'un de ces animaux afin d'avoir sous les yeux par l'oscillation de la colonne mercurielle l'expression des battements du cœur (fig. 20), on constate qu'au moment où l'on excite rapidement un nerf sensitif il y a arrêt du cœur en diastole, ce qui détermine une suspension de l'oscillation avec abaissement léger de la colonne mercurielle. Aussitôt après, les battements reparaissent considérablement accélérés et plus énergiques, car le mercure s'élève quelquefois de plusieurs centimètres pour redescendre à son point primitif lorsque le cœur calmé a repris son rhythme normal. 
Le cœur est quelquefois si sensible chez certains animaux que des excitations très-légères des nerfs sensitifs peuvent amener des réactions, lors même que l'animal ne manifeste aucun signe de douleur. Ce sont là des expériences

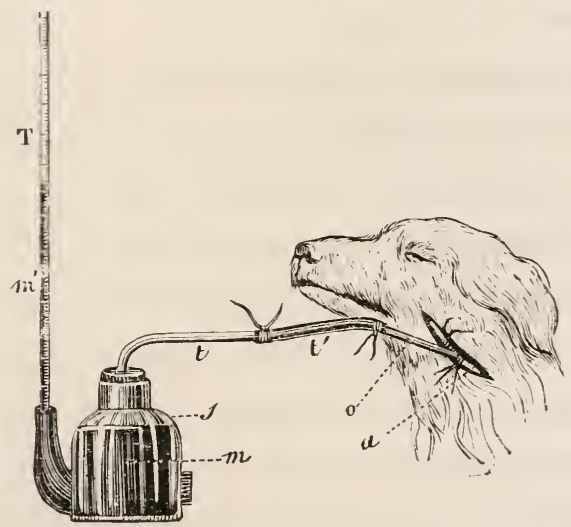

Fig. 20. Chien curarisé par ingestion. - La branche horizontale d'un manomètre est engagée dans l'artère carotide '.

que nous avons faites, mon maître Magendie et moi, il y a bien longtemps, et qui depuis ont été souvent répétẻes et vérifiées par des procédés divers.

1. $a$, carotide; $m$, mercure; $m^{\prime}$, mercure dans le tube ; $\mathrm{T}$, branche verticale; $t, t^{\prime}$, branche horizontale. 
A mesure que l'organisation animale s'élève, le cœur devient donc un réactif de plus en plus délicat pour trahir les impressions sensitives qui se passent dans le corps, et il est naturel de penser que l'homme doit être au premier rang sous ce rapport. Chez lui, le cœur n'est plus seulement l'organe central de la circulation du sang, mais il est devenu en outre un centre où viennent retentir toutes les actions nerveuses sensitives. Les influences nerveuses qui réagissent sur le cœur arrivent soit de la périphérie par le système cérébro-spinal, soit des organes intérieurs par le grand sympathique, soit du centre cérébral lui-même, car au point de vue physiologique il faut considérer le cerveau comme la surface nerveuse la plus délicate de toutes : d'où il résulte que les actions sensitives qui proviennent de cette source sont celles qui exerceront sur le cœur les influences les plus énergiques. 


\section{IV}

Comment est-il possible de concevoir le mécanisme physiologique à l'aide duquel le cœur se lie aux manifestations de nos sentiments?

Nous savons que cet organe peut recevoir le contre-coup de toutes les vibrations sensitives qui se passent en nous, et qu'il peut en résulter tantôt un arrêt violent avec suspension momentanée et ralentissement de la circulation, si l'impression a été très-forte, tantôt un arrêt léger avec réaction et augmentation du nombre et de l'énergie des battements cardiaques, si l'impression a été légère ou modérée; mais comment cet état peut-il ensuite traduire nos sentiments? C'est ce qu'il s'agit d'expliquer.

Rappelons-nous que le cœur ne cesse jamais d'être une pompe foulante, c'est-à-dire un moteur qui distribue le liquide vital à tous les or- 
ganes de notre corps. S'il s'arrête, il y a nécessairement suspension ou diminution dans l'arrivée du liquide vital aux organes, et par suite suspension ou diminution de leurs fonctions; si au contraire l'arrêt léger du cœur est suivi d'une intensité plus grande dans son action, il y a distribution d'une plus grande quantité du liquide vital dans les organes, et par suite surexcitation de leurs fonctions.

Cependant tous les organes du corps et tous les tissus organiques ne sont pas également sensibles à ces variations de la circulation artérielle, qui peuvent diminuer ou augmenter brusquement la quantité du liquide nourricier qu'ils reçoivent. Les organes nerveux et surtout le cerveau, qui constituent l'appareil dont la texture est la plus délicate et la plus élevée dans l'ordre physiologique, recoivent les premiers les atteintes de ces troubles circulatoires. C'est une loi générale pour tous les animaux : depuis la grenouille jusqu'à l'homme, la suspension de la circulation du sang amène en premier lieu la perte des fonctions cérébrales et nerveuses, de même que l'exagération de la circula- 
tion exalte d'abord les manifestations cérébrales et nerveuses.

Toutefois ces réactions de la modification circulatoire sur les organes nerveux demandent pour s'opérer un temps très-différent selon les espèces.

Chez les animaux à sang froid, ce temps est très-long, surtout pendant l'hiver; une grenouille reste plusieurs heures avant d'éprouver les conséquences de l'arrêt de la circulation; on peut lui enlever le cœur, et pendant quatre ou cinq heures elle saute et nage sans que sa volonté ni ses mouvements paraissent le moins du monde troublés.

Chez les animaux à sang chaud, c'est tout différent : la cessation d'action du cœur amène très-rapidement la disparition des phénomènes cérébraux, et d'autant plus facilemeut que l'animal est plus élevé, c'est-à-dire possède des organes nerveux plus délicats.

Le raisonnement et l'expérience nous montrent qu'il faut encore placer, sous ce rapport, l'homme au premier rang. Chez lui, le cerveau est si délicat qu'il éprouvera en quelques se- 
condes, et pour ainsi dire instantanément, le retentissement des influences nerveuses exercées sur l'organe central de la circulation, influences qui se traduisent comme nous allons le voir bientôt, tantôt par une émotion, tantôt par une syncope.

Les phénomènes physiologiques suivent partout une loi identique, mais la nature plus ou moins délicate de l'organisme vivant peut leur donner une expression toute différente. Ainsi la loi de réaction du cœur sur le cerveau est la même chez la grenouille et chez l'homme; cependant jamais la grenouille ne pourra éprouver une émotion ni une syncope, parce que le temps qu'il faut à son cœur pour ressentir l'influence nerveuse, et à son cerveau pour éprouver l'influence circulatoire, est si long que la relation physiologique entre les deux organes disparaît.

Chez l'homme, l'influence du cœur sur le cerveau se traduit par deux états principaux entre lesquels on peut supposer beaucoup d'intermédiaires : la syncope et l'émotion.

$\because$ La syncope est due à la cessation momen- 
tanée des fonctions cérébrales par cessation de l'arrivée du sang artériel dans le cerveau.

On pourrait produire la syncope en liant ou en comprimant directement toutes les artères qui vont au cerveau; mais ici ne nous occupons que de la syncope qui survient par une influence sensitive portée sur le cœur et assez énergique pour arrêter ses mouvements. L'arrêt du cœur qui produit la perte de connaissance en privant le cerveau du sang amène aussi la pâleur des traits et une foule d'autres effets accessoires dont il ne peut être question ici. Toutes les impressions sensitives énergiques et subites sont dans le cas d'amener la syrcope, quelle qu'en soit d'ailleurs la nature. Des impressions physiques sur les nerfs sensitifs ou des impressions morales, des sensations douloureuses ou des sensations de volupté, conduisent au même résultat et amènent l'arrêt du cœur.

La durée de la syncope est naturellement liée à la durée de l'arrêt du cœur. Plus l'arrêt a été intense, plus en général la syncope se prolonge, et plus difficilement se rétablissent les battements cardiaques, qui d'abord reviennent 
irrégulièrement pour ne reprendre que lentement leur rhythme normal.

Quelquefois l'arrêt du cœur est définitif et la syncope mortelle; chez les individus faibles et en même temps très-sensibles, cela peut arriver. On a constaté expérimentalement que, 'sur des colombes épuisées par l'inanition, il suffit parfois de produire une douleur vire, en pincant un nerf de sentiment, pour amener un arrêt du cœur définitif et une syncope mortelle.

L'émotion dérive du même mécanisme physiologique que la syncope, mais elle a une manifestation bien différente. La syncope, qui enlève le sang au cerveau, donne une expression négative, en prouvant seulement qu'une impression nerveuse violente est allée se réfléchir sur le cœur pour revenir frapper le cerveau. L'émotion au contraire, qui envoie au cerveau une circulation plus active, donne une expression positive, en ce sens que l'organe cérébral recoit une surexcitation fonctionnelle en harmonie avec la nature de l'influence nerveuse qui l'a déterminée. Dans l'émotion, il y a toujours une impression initiale qui surprend en 
quelque sorte et arrète très-légèrement le cœur, et par suite une faible secousse cérébrale qui amène une pâleur fugace; aussitôt le cœur, comme un animal piqué par un aiguillon, réagit, accélère ses mouvements et envoie le sang à plein calibre par l'aorte et par toutes les artères. Le cerveau, le plus sensible de tous les organes, éprouve immédiatement et avant tous les autres les effets de cetle modification circulatoire. Le cerveau a été sans doute le point de départ de l'impression nerveuse sensitive; mais par l'action réflexe sur les nerfs moteurs du cœur l'influence sensitive a provoqué dans le cerveau les conditions qui viennent se lier à la manifestation du sentiment.

En résumé, chez l'homme, le coeur est le plus sensible des organes de la vie végétative; il recoit le premier de tous l'influence nerveuse cérébrale. Le cerveau est le plus sensible des organes de la vie animale; il recoit le premier de tous l'influence de la circulation du sang. De là résulte que ces deux organes culminants de la machine vivante sont dans des rapports incessants d'action et de réaction. Le 
cœur et le cerveau se trouvent dès lors dans une solidarité d'actions réciproques des plus intimes, qui se multiplient et se resserrent d'autant plus que l'organisme devient plus développé et plus délicat.

Ces rapports peuvent être constants ou passagers, varier avec le sexe et avec l'âge. C'est ainsi qu'à l'époque de la puberté, lorsque des organes, jusqu'alors restés inertes ou engourdis, s'éveillent et se développent, des sentiments nouveaux prennent naissance dans le cerveau et apportent au cœur des impressions nouvelles.

Les sentiments que nous éprouvons sont toujours accompagnés par des action réflexes du cœur; c'est du cœur que viennent les conditions de manifestation des sentiments, quoique le cerveau en soit le siége exclusif. Dans les organismes élevés, la vie n'est qu'un échange continuel entre le système sanguin et le système nerveux. L'expression de nos sentiments se fait par un échange entre le cœur et le cerveau, les deux rouages les plus parfaits de la machine vivante. Cet échange se réalise par des relations 
anatomiques très-connues, par les nerfs pneumo-gastriques qui portent les influences nerveuses au cœur, et par les artèrus carotides et vertébrales qui apportent le sang au cerveau. Tout ce mécanisme merveilloux ne tient donc qu'à un fil, et si les nerfs qui unissent le cœur au cerveau venaient à ître détruits, cette réciprocité d'action serait interrompue, et la manifestation de nos suntiments profondément troublée.

Toutes ces explications, me dira-t-on, sont bien empreintes rle matérialisme.

A cela je répondrai que ce n'est pas ici la question. Si ce n'était m'écarter du but de ces recherches, je pourrais montrer facilement qu'en physiologie le matérialisme ne conduit à rien et n'explique rien; mais un concert en est-il moins ravissant parce que le physicien en calcule mathématiquement toutes les vibrations? Un phénomène physiologique en est-il muins admirable parce que le physiologiste en analyse toutes les conditions matérielles? Il faut bien que cette analyse, que ces calculs se fassent, car sans cela il n'y aurait pas de GLAUDE BERNARD. 
science. Or la science physiologique nous apprend que, d'une part, le cœur recoit réellement l'impression de tous nos sentiments, et que, d'autre part, le cœur réagit pour renvoyer au cerveau les conditions nécessaires dela manifestation de ces sentiments, d'où il résulte que le poëte et le romancier qui, pour nous émouvoir, s'adressent à notre cœur, que l'homme du monde qui à tout instant exprime ses sentiments en invoquant son cœur, font des métaphores qui correspondent à des réalités physiologiques.

Quelquefois un mot, un souvenir, la vue d'un événement, éveillent en nous une douleur pro fonde. Ce mot, ce souvenir ne sauraient être douloureux par eux-mêmes, mais seulement par les phénomènes qu'ils provoquent en nous.

Quand on dit que le cour est brisé par la douleur, il y a des phénomènes réels dans le cœur. Le cœur a été arrêté, si l'impression douloureuse a été trop soudaine; le sang n'arrivant plus au cerveau, la syncope, des crises nerveuses en sont la conséquence. On a donc bien raison, quand il s'agit d'apprendre à quelqu'un 
une de ces nouvelles terribles qui bouleversent notre âme, de ne la lui faire connaître qu'avec ménagement.

Nous savons par nos expérierces sur les nerfs du cour que les excitations graduées émoussent ou épuisent la sensibilité cardiaque en évitant l'arrêt des battements.

Quand on dit qu'on a le cocur gros, après avoil longtemps été dans l'angoisse et avoir éprouvé des émotions pénibles, cela répond encore à des conditions physiologiques particulières du cœur. Les impressions douloureuses prolongées, devenues incapables d'arrêter le cœur, le fatiguent et le lassent, retardent ses battements, prolongent la diastole, et font éprouver dans la région précordiale un sentiment de plénitude ou de resserrement.

Les impressions agréables répondent aussi à des états déterminés du cœur.

Quand une femme est surprise par une douce émotion, les paroles qui ont pu la faire naître ont traversé l'esprit comme un éclair, sans s'y arrêter; le cœur a été alteint immédiatement et avant tout raisonnement et toute réflexion. Le 
sentiment commence à se manifester après un léger arrêt du cœur, imperceptible pour tout le monde, excepté pour le physiologiste; le cœur, aiguillonné par l'impression nerreuse, réagit par des palpitations qui le font bondir et battre p̉lus fortement dans la poitrine, en même temps qu'il envoie plus de sang au cerveau, d'où résultent la rougeur du visage et une expression particulière des traits correspondant au sentiment de bien-être éprouvé.

Ainsi dire que l'amour fait palpiter le cœur n'est pas seulement une forme poétique; c'est aussi une réalité physiologique.

Quand on dit à quelqu'un qu'on l'aime de tout son cœur, cela signifie physiologiquement que sa présence ou son souvenir éveille en nous une impression nerveuse qui, transmise au cœur par les nerfs pneumo-gastriques, fait réagir notre cœur de la manière la plus convenable pour provoquer dans notre cerveau un sentiment ou une émotion affective. Je suppose ici, bien entendu, que l'aveu est sincère; sans cela, le cœur n’éprouverait rien et le sentiment ne serait que sur les lèvres. 
Chez l'homme, le cerveau doit, pour exprimer ses sentiments, avoir le cœur à son service.

Deux cœurs unis sont des cœurs qui battent à l'unisson sous l'influence des mêmes impres. sions nerveuses, d'où résulte l'expression harmonique de sentiments semblables.

Les philosophes disent qu'on peut maîtriser son cœur et faire taire ses passions. Ce sont encore des expressions que la physiologie peut interpréter. On sait que par sa volonté l'homme peut arriver à dominer beaucoup d'actions réflexes dues à des sensations produites par des causes physiques. La raison parvient sans doute à exercer le même empire sur les sentiments moraux. L'homme peut arriver par la raison à empêcher les actions réflexes sur son cœur; mais plus la raison pure tendrait à triompher, plus le sentiment tendrait à s'éteindre.

La puissance nerveuse capable d'arrêter les actions réflexes est en général moindre chez la femme que chez l'homme: c'est ce qui lui donne la suprématie dans le domaine de la 
sensibilité physique et morale, c'est ce qui a fait dire qu'elle a le cœur plus tendre que l'homme.

Mais je m'arrête dans ces considérations, qui nous entraîneraient trop loin, et je terminerai par une conclusion générale.

La science ne contredit point les observations et les données de l'art, et je ne saurais admettre l'opinion de ceux qui croient que le positivisme scientifique doit tuer l'inspiration. Suivant moi, c'est le contraire qui arrivera nécessairement. L'artiste trouvera dans la science des bases plus stables, et le savant puisera dans l'art une intuition plus assurée. Il peut sans doute exister des époques de crise dans lesquelles la science, à la fois trop avancée et encore trop imparfaite, inquiète et trouble l'artiste plutôt qu'elle ne l'aide. C'est ce qui peut arriver aujourd'hui pour la physiologie à l'égard du poëte et du philosophe; mais ce n'est là qu'un état transitoire, et j'ai la conviction que quand la physiologie sera assez avancée, le poëte, le philosophe et le physiologistes'entendront tous.

Revue des Deux-Mondes, 1 ${ }^{\text {er }}$ mars 1865. 


\section{DES FONGTIONS DU GERVEAU}

I

Le premier soin de la physiologie a été de localiser les fonctions de la vie dans les différents organes du corps qui leur servent d'instruments.

C'est ainsi qu'on a rattaché la digestion à l'estomac, la circulation au cœur, la respiration au poumon; c'est encore de même qu'on a placé le siége de l'intelligence et de la pensée dans le cerveau.

Toutefois, relativement à ce dernier organe, on a cru devoir faire des réserves et ne pas ad- 
mettre que l'expression métaphysique des facultés intellectuelles et morales fût la manifestation pure et simple de ía fonction cérébrale.

Descartes, qu'il f:xut mettre au nombre des promoteurs de la physiologie moderne, parce qu'il a très-bien compris que les explications des phénomènes de la vie ne peuvent relever que des lois de la physique et de la mécanique générales, s'est clairement exprimé à cet égard. Adoptant les idées de Galien sur la formation des esprits animaux dans le cerveau, il leur donne pour mission de se répandre au moyen des nerfs dans toute la machine animée, afin de porter à chacune des parties l'impulsion nécessaire à son activité spéciale. Cependant, audessus et distincte de cette fonction physiologique du cerveau, Descartes admet l'âme, qui donne à l'homme la faculté de penser; elle aurait son siége dans la glande pinéale, et dirigerait les esprits animaux qui en émanent et lui sont subordonnés.

Les opinions de Descartes touchant les fonctions du cerveau ne pourraient aujourd'hui 
supporter le moindre examen physiologique; ses explications, fondées sur des connaissances anatomiques insuffisantes, n'ont pu enfanter que des hypothèses empreintes d'un grossier mécanisme. Néanmoins elles ont pour nous une valeur historique, elles nous montrent que ce grand philosophe reconnaissait dans le cerveau deux choses: d'abord un mécanisme physiologique, puis, au-dessus et en dehors de lui, la faculté pensante de l'âme.

Ces idées sont à peu près celles qui ont régné ensuite parmi beaucoup de philosophes et parmi certains naturalistes; le cerveau, où s'accomplissent les fonctions les plus importantes $\mathrm{du}$ système nerveux, serait non pas l'organe réel de la pensée, mais seulement le substratum de l'intelligence. Bien souvent en effet on entend faire cette objection, que le cerveau forme une exception physiologique à tous les autres organes du corps, en ce qu'il est le siégre de manifestations métaphysiques qui ne sont pas du ressort du physiologiste. On concoit que l'on puisse ramener la digestion, la respiration, la locomotion, etc., à des phénomènes de mécani- 
que, de physique et de chimie; mais on n'admet pas que la pensée, l'intelligence, la volonté se soumettent à de semblables explications. Il y a là, dit-on, un abîme entre l'organe et la fonction, parce qu'il s'agit de phénomènes métaphysiques et non plus de mécanismes physicochimiques.

De Blainville, dans ses cours de zoologie, insistait beaucoup sur la définition de l'organe et du substratum. "Dans l'organe, disait-il, il y a un rapport visible et nécessaire entre la structure anatomique et la fonction; dans le cœur, organe de la circulation, la conformation et la disposition des orifices et de leurs valvules rend parfaitement compte de la circulation du sang. Dans le substratum, rien de pareil ne s'observe: le cerveau est le substratum de la pensée; elle a son siége en lui, mais la pensée ne saurait se déduire de l'anatomie cérébrale. »

C'est en se fondant sur de pareilles considérations qu'on s'est cru autorisé à prétendre que la raison pouvait être, chez les aliénés, troublée d'une manière dite essentielle, c'est-àdire sans qu'il existât aucune lésion matérielle 
du cerveau. La réciproque a été de même soutenue, et on trouve cités dans des traités de physiologie des cas où l'intelligence se serait manifestée intègre chez des individus dont le cerveau était ramolli ou pétrifié.

Aujourd'hui les progrès de la science ont ruiné toutes ces doctrines; cependant il faut reconnaître que les physiologistes qui se sont autorisés des recherches modernes les plus délicates sur la structure du cerveau pour localiser la pensée dans une substance particulière ou dans des cellules nerveuses d'une forme et d'un ordre déterminés n'ont pas davantage résolu la question, car ils n'ont fait en réalité qu'opposer des hypothèses matérialistes à d'autres hypothèses spiritualistes.

De tout ce qui précède, je tirerai la seule conclusion légitime qui en découle : c'est que le mécanisme de la pensee nous est inconnu, et je crois que tout le monde sera d'accord sur ce point.

La question fondamentale que nous arons posée n'en subsiste pas moins, car ce qui nous importe, c'est de savoir si l'ignorance 
où nous sommes à ce sujet est une ignorance relative qui disparaîtra avec les progrès de la science, ou bien si c'est une ignorance absolue en ce sens qu'il s'agirait là d'un problème vital qui doit à jamais rester en dehors de la physiologie.

Je repousse, tant qu'à moi, cette dernière opinion, parce que je n'admets pas que la vérité scientifique puisse ainsi se fractionner. Comment comprendre en effet qu il soit donné au physiologiste de pouvoir expliquer les phénomènes qui s'accomplissent dans tous les organes du corps, excepté une partie de ceux qui se passent dans le cerveau? De semblables distinctions ne peuvent exister dans les phénomènes de la vie. Ces phénomènes présentent sans doute des degrés de complexité très-différents, mais ils sont tous au même titre accessibles ou inaccessibles à nos investigations, et le cerveau, quelque merveilleuses que nous paraissent les manifestations métaphysiques dont il est le siége, ne saurait constituer une exception farmi les autres organes du corps. 


\section{II}

Les phénomènes métaphysiques de la pensee, de la conscience et de l'intelligence, qui servent aux manifestations diverses de l'âme humaine, considérés au point de vue physiologique, ne sont que des phénomènes ordinaires de la vie, et ne peuvent être que le résultat de la fonction de l'organe qui les exprime.

Nous allons montrer en effet que la physiologie du cerveau se déduit, comme celle de tous les autres organes du corps, des observations anatomiques, de l'expérimentation physiologique et des connaissances de l'anatomie pathologique.

Dans son déreloppement anatomique, le cerveau suit la lói commune, c'est-à-dire qu'il devient plus volumineux quand les fonctions 
auxquelles il préside augmentent de puissance ${ }^{1}$. A mesure que l'intelligence se manifeste davantage, nous voyons dans la série des animaux le cerveau acquérir un plus grand développement, et c'est chez l'homme, où les phénomènes intellectuels sont arrivés à leur expression la plus élevée, que l'organe cérébral présente le volume le plus considérable.

D'après la forme du cerveau (fig. 21), d'après le nombre des plis ou circonvolutions qui en étendent la surface, on peut déjà préjuger l'intelligence des divers animaux; mais ce n'est pas seulement l'aspect extérieur du cerveau qui change quand ses fonctions se modifient, il offre en même temps dans sa structure intime une complexité qui s'accroît avec la variété et l'intensité des manifestations intellectuelles. Relativement à la texture du cerveau, nous n'en sommes plus au temps de Buffon, qui considéraitla cervelle, ainsi qu'il l'appelait avec dédain, comme une substance muqueuse sans importance.

1. Voy. Leuret et Gratiolet, Anatomie comparée du système nerveux. Paris, 1839-1857. 


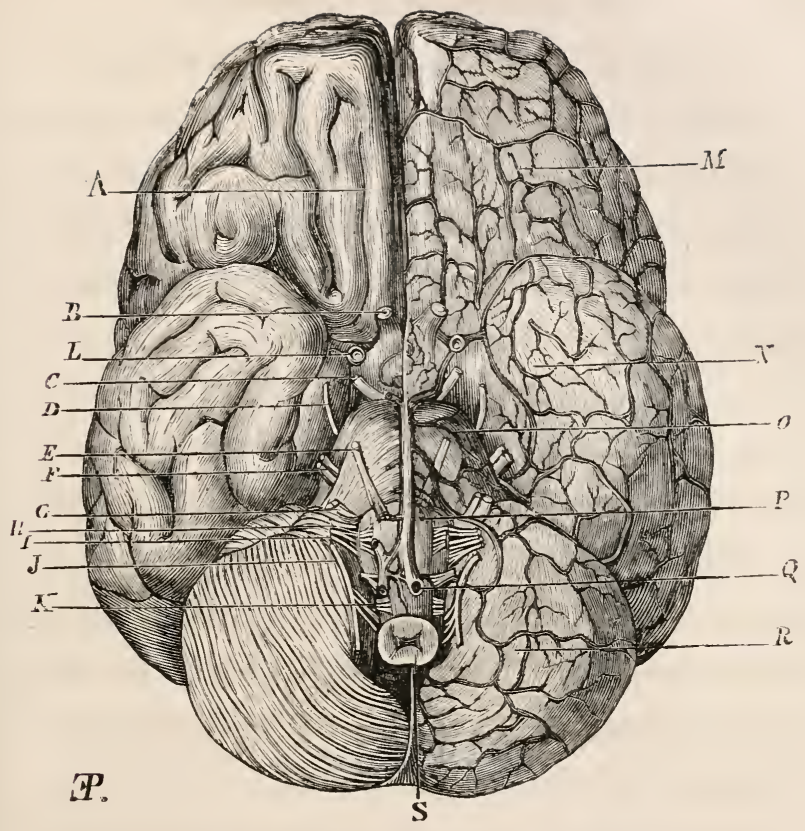

Fig. 21. Face inférieure du cerveau '.

1. A, sillon du nerf olfactif; le sillon existe beaucoup moins prononcé, mais on ne trouve aucun vestige de nerf olfactif, pas même vers son origine; B, nerf ophthalmique; $\mathrm{C}$, nerf moteur oculaire commun; D, nerf pathétique; $\mathrm{E}$, moteur oculaire externe; F, nerf trijumeau; $G$, nerf de la septième paire; $H$, nerf vague; $I$, nerf glosso-pharyngien; J, nerf spinal; K, nerf grand hypoglosse; L, artère carotide interne; $\mathrm{M}$, lobe antérieur du cerveau; $\mathrm{N}$, lobe cérẻbral moyen; $\mathrm{O}$, pont de Varole ; $\mathrm{P}$, artère basilaire; $Q$, pyramide antérieure; $\mathrm{R}$, cervelet. 
Les proggrès de l'anatomie générale et de l'histologie nous ont appris que l'organe cérébral possède la texture à la fois la plus délicate et la plus complexe de tous les appareils nerveux. Les éléments anatomiques qui le composent sont des éléments nerveux sous la forme de tubes et de cellules combinés et unis entre eux. Ces éléments sont semblables dans tous les animaux par leurs propriétés physiologiques et par leurs caractères histologiques; ils diffèrent par le nombre, les réseaux, les connexions, l'arrangement en un mot, qui présente une disposition particulière dans le cerveau de chaque espèce.

En cela, le cerveau suit encore la loi générale, car dans tous İes organes l'élément anatomique garde des caractères fixes qui le font reconnaître; le perfectionnement organique consiste surtout dans l'arrangement de ces éléments, qui, dans chaque espèce animale, offre une forme spécifique. Chaque organe serait donc en réalité un appareil dont les éléments constitutifs restent identiques, mais dont le groupement devient de plus en plus compliqué à mesure que 
la fonction elle-même se montre plus variée et plus complexe.

Si nous considérons maintenant les conditions organiques et physico-chimiques nécessaires à l'entretien de la vie et à l'exercice des fonctions, nous verrons qu'elles sont les mêmes dans le cerveau que dans tous les autres organes.

Le sang agit sur les éléments anatomiques de tous les tissus en leur apportant les conditions de nutrition, de température, d'humidité, qui leur sont indispensables. Lorsque le sang afflue en moindre quantité dans un organe quelconque, l'activité fonctionnelle se modère, et l'organe entre au repos; mais, si le fluide sanguin est supprimé, les propriétés élémentaires du tissu s'altèrent peu à peu, en même temps que les fonctions sont anéanties.

Il en est absolument de même pour les éléments anatomiques du cerveau. Dès que le sang cesse d'y parvenir, les propriétés nerveuses sont atteintes, ainsi que les fonctions cérébrales, qui finissent par disparaître, si l'anémie devient complète. Une simple modification dans la 
température du sang, dans sa pression, suffit pour produire des troubles profonds dans la sensibilité, le mouvement ou la volonté.

Tous les organes du corps nous offrent alternativement un état de repos et un état de fonction dans lesquels les phénomènes circulatoires sont essentiellement différents.

Des observations nombreuses, prises dans les appareils les plus divers, ont mis ces faits hors de doute.

Lorsque par exemple on examine le canal alimentaire d'un animal à jeun, on trouve la membrane muqueuse qui revêt la face interne de l'estomac et des intestins pâle et peu vascularisée; pendant la digestion au contraire, on constate que la même membrane est très-colorée et gonflée par le sang, qui y afflue avec force. Ces deux phases circulatoires, à l'état de repos et à l'état de fonctions, ont pu être véri-' fiées directement dans l'estomac chez l'homme vivant.

Tous les physiologistes connaissent l'histoire d'un jeune Canadien blessé accidentellement d'un coup de mousquet chargé à plomb qui 
l'atteignit presque à bout portant dans le flanc gauche ${ }^{1}$. La cavité abdominale avait été ouverte par une énorme plaie contuse, et l'estomac, largement perforé, laissait échapper les aliments du dernier repas. Le malade fut soigné par le docteur Beaumont, chirurgien à l'armée des États-Unis; il guérit, mais en conservant une plaie fistuleuse de trente-cinq à quaranțe millimètres de circonférence, à travers laquelle on pouvait introduire différents corps et inspecter facilement ce qui se passait dans l'estomac. Le docteur Beaumont, voulant étudier ce cas remarquable, s'attacha en qualité de domestique ce jeune homme, dont la santé et les facultés digestives en particulier s'étaient complétement rétablies. Il put le garder à son service pendant sept années, durant lesquelles il fit un très-grand nombre d'observations du plus haut intérêt pour la physiologie. A jeun, en regardant dans l'intérieur de l'estomac, on en apercevait distinctement la membrane interne; elle formait des

1. Voy. Claude Bernard, Lecons de physiologie expérimentale appliquée à la médecine. Paris, 1856, tome II, p. 382. 
replis irréguliers, la surface, d'un rose pâle, n’était animée d'aucun mouvement, et n'était absolument lubrifiée que par du mucus. Aussitôt que les matières alimentaires descendaient dans l'estomac et touchaient la membrane muqueuse, la circulation s'y accélérait, la couleur s'avivait, et des mouvements péristaltiques s'y manifestaient. Les papilles muqueuses versaient alors le suc gastrique, fluide clair et transparent destiné à dissoudre les aliments. Lorsqu'on essuyait avec une éponge ou un linge fin le mucus qui recouvrait la membrane villeuse, on voyait bientôt le suc gastrique reparaître et s'assembler en gouttelettes qui ruisselaient le long des parois de l'estomac comme la sueur sur le visage.

Ce que nous venons de voir sur la membrane muqueuse gastrique s'observe de même pour tout l'intestin et pour tous les organes glandulaires annexés à l'appareil digestif.

Les glandes salivaires, le pancréas, pendant l'intervalle des digestions, présentent un tissu pâle et exsangue dont les sécrétions sont entièrement suspendues. Pendant la période di- 
gestive au contraire, ces mêmes glandes sont gorgées de sang, rutilantes, comme érectiles, et leurs conduits laissent écouler les liquides sécrétés en abondance.

Il faut donc reconnaître dans les organes deux ordres de circulations : d'un côté la circulation générale, connue depuis Harvey, et de l'autre les circulations locales, découvertes et étudiées seulement dans ces derniers temps. Dans les phénomènes de circulation générale, le sang ne fait en quelque sorte que traverser les parties pour passer des artères dans les veines; dans les phénomènes de la circulation locale, qui est lavraie circulation fonctionnelle, le fluide sanguin pénètre dans tous les replis de l'organe, et s'accumule autour des éléments anatomiques pour réveiller et exciter leur mode d'activité spéciale.

Le système nerveux, sensitif et vaso-moteur, préside à tous les phénomènes de circulations locales qui accompagnent les fonctions organiques; c'est ainsi que la salive s'écoule abondamment lorsqu'un corps sapide vient impressionner les nerfs de la membrane muqueuse 
buccale, et que le suc gastrique se forme sous l'influence du contact des aliments et de la surface sensible de l'estomac. Toutefois cette excitation mécanique sur les nerfs sensitifs périphériques, venant retentir sur l'organe par action réflexe, peut être remplacée par une excitation purement psychique ou cérébrale.

Une expérience simple vient en donner la démonstration.

Prenant un cheval à jeun, on découvre sur le côté de la mâchoire le canal excréteur de la glande parotide, on divise ce conduit, et rien n'en sort; la glande est au repos. Si alors on fait voir au cheval de l'avoine, ou mieux, si, sans rien lui montrer, on exécute un mouvement qui indique à l'animal qu'on va lui donner son repas, aussitôt un jet continu de salive s'écoule du conduit parotidien, en même temps que lo tissu de la glande s’injecte et devient le siége d'une circulation plus active.

Le docteur Beaumont a observé sur son Canadien des phénomènes analogues. L'idée d'un mets succulent déterminait non-seulement un appel de sécrétion dans les glandes salivaires, 
mais provoquait encore un afflux sanguin immédiat sur la membrane muqueuse stomacale.

Ce que nous venons de dire sur les circulations locales ou fonctionnelles ne s'applique pas seulement aux organes sécréteurs où s'opère la séparation d'un liquide à la formation duquel le sang doit plus ou moins concourir; il s'agit là d'un phénomène général qui s'observe dans tous Jes organes, quelle que soit la nature de leur fonction.

Le système musculaire, qui ne produit qu'un travail mécanique, est dans le même cas que les glandes, qui agissent chimiquement. Au moment de la fonction du muscle, le sang circule avec une plus grande activité, qui se modère quand l'organe entre en repos.

Le système nerveux périphérique, la moelle épinière et le cerveau, qui servent à la manifestation des phénomènes de l'innervation et de l'intelligence, n'échappent pas non plus à cette loi, ainsi que nous allons le voir.

Les relations qui existent entre les phénomènes circulatoires du cerveau et l'activité fonctionnelle de cet organe ont été longtemps ob- 
scurcies par des opinions erronées sur les con-. ditions du sommeil, considéré à juste titre comme l'état de repos de l'organe cérébral.

Les anciens croyaient que l'état de sommeil était la conséquence d'une compression opérée sur le cerveau par le sang lorsque sa circulation se ralentit. Ils supposaient que cette pression s'exerçait surtout à la partie postérieure de la tête, au point où les sinus veineux de la dure-mère viennent aboutir dans un confluent commun qu'on appelle encore torcular ou pressoir d'Hérophile, du nom de l'anatomiste qui en donna la première description. Ces explications hypothétiques se sont transmises jusqu'à nous.

Ce n'est que dans ces dernières années que l'expérimentation est venue en démontrer la fausselé. On a prouvé en effet par des expériences directes que pendant le sommeil le cerveau, au lieu d'être congestionné, est au contraire pâle et exsangue, tandis que pendant la veille la circulation, devenue plus active, provoque un afflux de sang qui est en raison de l'intensité des fonctions cérébrales. Sous ce rapport, le sommeil naturel et le sommeil anesthésique du 
chloroforme se ressemblent; dans les deux cas, le cerveau, plongé dans le repos ou l'inaction, présente la même pâleur et la 'mème anémie relative.

Voici comment se fait l'expérience.

Sur un animal, on enlève avec soin une partie de la paroi osseuse du crâne, et on met à nu le cerveau de manière à observer la circulation à la surface de cet organe. C'est alors qu'on fait respirer du chloroforme pour opérer l'anesthésie. Dans la première période excitante de l'action chloroformique, on voit le cerveau se congestionner et faire hernie au dehors; mais, dès que la période du sommeil anesthésique arrive, la substance cérébrale s'affaisse, pâlit, en présentant un affaiblissement de la circulation capillaire qui persiste autant que dure l'état de sommeil ou de repos cérébral.

Pour observer le cerveau pendant le sommeil naturel, on a pratiqué sur des chiens des couronnes de trépan en remplacant la pièce osseuse enlevée par un verre de montre exactement appliqué, afin d'empêcher l'action irritante de l'air extérieur. Les animaux survivent parfaite- 
ment à cette opération; en observant leur cerveau par cette sorte de fenêtre pendant la veille et pendant le sommeil, on constate que lorsque le chien dort, le cerveau est toujours plus pâle, et qu'un nouvel afflux sanguin se manifeste constamment au réveil, lorsque les fonctions cérébrales reprennent leur activité.

Des faits analogues à ceux observés chez les animaux ont été vus directement sur le cerveau de l'homme.

Sur un individu victime d'un épouvantable accident de chemin de fer, on eut l'occasion d'observer une perte de substance considérable. Le cerveau apparaissait dans une étendue de trois pouces de long sur six de large. Le blessé présentait de fréquentes et graves attaques d'épilepsie et de coma, pendant lesquelles le cerveau s'élevait invariablement. Après ces atta= ques, le sommeil survenait, et la hernie cérébrale s'affaissait graduellement. Lorsque le malade était réveillé, le cerveau faisait de nouveau saillie, et se mettait de niveau avec la surface de la table externe de l'os.

A la suite d'ıne fracture du cràne, on observa 
chez un autre blessé la circulation cérébrale pendant l'administration des anesthésiques. Au début de l'inhalation, la surface cérébrale devenait arborescente et injectée; l'hémorrhagie et les mouvements du cerveau augmentaient, puis, au moment du sommeil, la surface du cerveau s'affaissait peu à peu au-dessous de l'ouverture, en même temps qu'elle devenait relativement pâle et anémiée.

En résumé, le cerveau est soumis à la loi commune qui régit la circulation du sang dans tous les organes. En vertu de cette loi, quand les organes sommeillent et que les fonctions en sont suspendues, la circulation y devient moins active; elle augmente au contraire dès que la fonction vient à se manifester. Le cerveau, je le répète, ne fait pas exception à cette loi générale, comme on l'avait cru, car il est prouvé aujourd'hui que l'état de sommeil coïncide non pas avec la congestion, mais au contraire avec l'anémie du cerveau.

Si maintenant nous cherchons à comprendre les relations qui peuvent exister entre la suractivité circulatoire du sang et l'état fonctionnel 
des organes, nous verrons facilement que cet afflux plus considérable du liquide sanguin est en rapport avec une plus grande intensité dans les métamorphoses chimiques qui s'opèrent au sein des tissus, ainsi qu'avec un accroissement dans les phénomènes caloriques qui en sont la conséquence nécessaire et immédiate.

La production de la chaleur dans les êtres vivants est un fait constaté dès la plus haute antiquité; mais les anciens eurent des idées fausses sur l'origine de la chaleur : ils l'attribuèrent à une puissance organique innée ayant son siége dans le cœur, foyer où bouillonnent le sang et les passions.

Plus tard, le poumon fut considéré comme une sorte de calorifère dans lequel la masse du sang venait tour à tour puiser la chaleur que la circulation était chargée de distribuer à tout le corps.

Les progrès de la physiologie moderne ont prouvé que toutes ces localisations absolues des conditions de la vie sont des chimères. Les sources de la chaleur animale sont partout et nulle part d'une manière exclusive. Ce n'est 
que par l'harmonisation fonctionnelle des divers organes que la température se maintient à peu près fixe chez l'homme et les animaux à sang chaud. Il y a en vérité autant de foyers calorifiques qu'il y a d'organes et de tissus particuliers, et nous devons partout relier la production de chaleur avec le travail fonctionnel des organes. Quand un muscle se contracte, quand une surface muqueuse, une glande sécrètent, il y a invariablement production de chaleur en même temps qu'il se produit une suractivité dans les phénomènes circulatoires locaux ${ }^{1}$.

En est-il de même pour le système nerveux et pour le cerveau? Des expériences modernes ne permettent pas d'en douter. Chaque fois que la moelle épinière et les nerfs manifestent la sensibilité ou le mouvement, chaque fois qu'un travail intellectuel s'opère dans le cerveau, une quantité de chaleur correspondante s'y produit. Nous devons donc considérer la chaleur dans l'économie animale comme une résuitante du

1. Voy. Claude Bernard, Leçons sur la chaleur. $\mathrm{Pa}-$ ris, 1876. 
travail organique de toutes les parties du corps; mais en même temps elle devient aussi le principe de l'activité de chacune de ces parties. Cette corrélation est surtout indispensable pour le cerveau et le système nerveux, qui tiennent sous leur dépendance toutes les autres actions vitales. Les expériences ont montré que le tissu du cerveau présente la température la plus élevée de tous les organes du corps. Chez l'homme et les animaux à sang chaud, le cerveau produit lui-mème la chaleur qui est nécessaire à la manifestation de ses propriétés de tissu. S'il n'en était pas ainsi, il. se refroidirait infailliblement, et on verrait aussitôt toutes les fonctions cérébrales s'engourdir, l'intelligence et la volonté disparaître. C'est ce qui arrive chez les animaux à sang froid, chez lesquels la fonction de calorification n'est pas suffisante pour permettre à l'organisme de résister aux causes de refroidissement extérieures. 
Sous le rapport des conditions organiques ou physico-chimiques de ses fonctions, le cerrean ne nous présente done rien d'exceptionnel.

Si maintenant nous passons à l'expérimentation physiologique, nous verrons qu'elle parvient à analyser les phénomènes cérébraux de la même manière que ceux de tous les autres organes.

Le procédé expérimental le plus généralement mis en pratique pour déterminer les fonctions des organes consiste à les enlever ou à les détruire d'une facon lente ou brusque, afin de juger des usages de l'organe d'après les troubles spéciaux apportés dans les phénomènes de la vie. Ce procédé de destruction ou d'ablation organique, qui constitue une méthode brutale 
de vivisection, a été appliqué sur une grande échelle à l'étude de tout le système nerveux ${ }^{1}$.

Ainsi, quand on a coupé un nerf et que les parties auxquelles il se distribue perdent leur sensibilité, nous en concluons que c'est là un nerf de sensibilité; si c'est le mouvement qui disparaît, nous en inférons qu'il s'agit d'un nerf de mouvement.

On a employé la même méthode pour connaître les fonctions des diverses parties de l'organe encéphalique, et, bien qu'on ait rencontré ici de nouvelles difficultés d'exécution à cause de la complexité des parties, cette méthode a fourni des résultats généraux incontestables.

Tout le monde savait déjà que l'intelligence n'est pas possible sans cerveau, mais l'expérimentation a précisé le rôle qui revient à chacune des portions de l'encéphale. Elle nous apprend que c'est dans les lobes cérébraux que réside la conscience ou l'intelligence proprement dite, tandis que les parties inférieures de l'en-

1. Voy. Leçons sur la physiologie et la pathologie du système nerveux. Paris, 1858. 
céphale recèlent des centres nerveux affectés à des fonctions d'ordre inférieur.

Ce n'est pas ici le lieu de décrire le rôle particulier de ces différentes espèces de centres nerveux qui se superposent et s'échelonnent en quelque sorte jusque dans la moelle épinière, il suffit de constater que nous en devons la connaissance à la méthode de vivisection par ablation organique qui s'applique d'une manière générale à toutes les investigations physiologiques. Ici le cerveau se comporte encore de même que tous les autres organes du corps, en ce sens que chaque lésion de sa substance amène dans ses fonctions des troubles caractéristiques et correspondant toujours à la mutilation qui a été produite.

Au moyen des lésions cérébrales qu'il produit, le physiologiste ne se borne pas à provoquer des paralysies locales qui suppriment l'action de la volonté sur certains appareils organiques; il peut aussi, en rompant seulement l'équilibre des fonctions cérébrales, amener la suppression de la liberté dans les mouvements volontaires. C'est ainsi qu'en blessant les pédon- 
cules cérébelleux et divers points de l'encéphale, l'expérimentateur peut à son gré faire marcher un animal à droite, à gauche, en avant, en arrière, ou le faire tourner, tantôt par un mouvement de manége (fig. 22), tantôt par un mou-

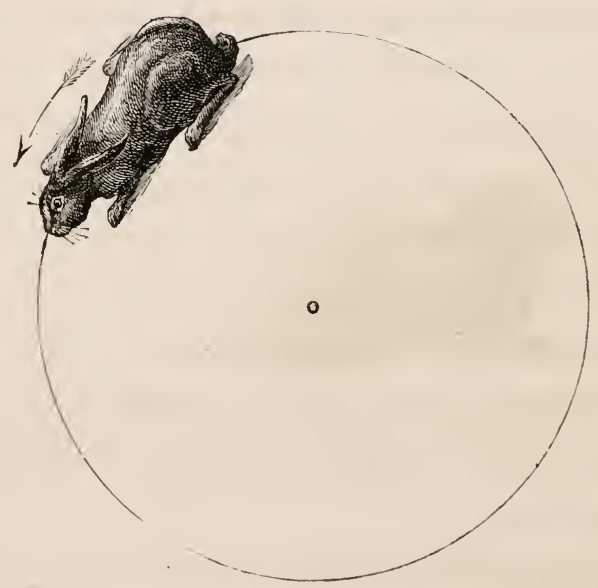

Fig. 22. Mouvements de manége.

vement de rotation sur l'axe de son corps (fig. 23). La volonté de l'animal persiste, mais il n'est plus libre de diriger ses mouvements. Malgré ses efforts de volonté, il va fatalement dans le sens que la lésion organique a déterminé. 
Les pathologistes ont signalé chez l'homme des faits analogues en grand nombre. Les lésions des pédoncules cérébelleux déterminent chez l'homme cornme chez les animaux les mouvements de rotation. D'autres malades ne pou-

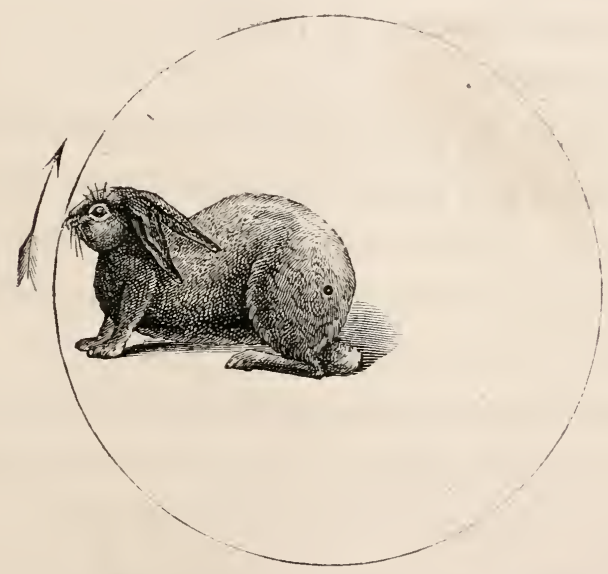

Fig. 23. Mouvement de rotation en rayon de roue.

vaient marcher que droit devant eux. Par une cruelle ironie, un brave et vieux général ne pouvait marcher qu'en reculant. La volonté qui part du cerveau ne s'exerce donc pas sur nos organes locomoteurs eux-mêmes; elle s'exerce sur 
des centres nerveux secondaires qui doivent être pondérés par un équilibre physiologique parfait.

Il est une autre méthode expérimentale plus délicate, qui consiste à introduire dans le sang des substances toxiques diverses destinées à porter leur action sur les éléments anatomiques des organes laissés en place el conservés dans leur intégrité.

A l'aide de cette méthode, on peut éteindre isolément les propriétés de certains éléments nerveux et cérébraux de la même manière qu'on isole aussi les autres éléments organiques musculaires ou sanguins.

Les anesthésiques, par exemple, font disparaître la conscience et engourdissent la sensibilité en laissant la motricité intacte ${ }^{1}$.

Le curare au contraire détruit la motricité, et laisse dans leur intégrité la sensibilité et la volonté; les poisons du cœur abolissent la contractilité musculaire, l'oxyde de carbone détruit la propriété oxydante du globule san-

1. Voy. Claude Bernard, Leçons sur les anesthésiques et l'asphyxie. Paris, 1875. 
guin sans modifier en rien les propriétés des éléments nerveux ${ }^{1}$.

Comme on le voit, par cette méthode d'investigation ou d'analyse élémentaire des propriétés organiques, le cerveau et les phénomènes dont il est le siége peuvent encore être atteints de la même manière que tous les autres appitreils fonctionnels du corps.

Enfin il est une troisième méthode d'expérimentation, qu'on pourrait appeler celle des expériences par rédintégration.

Cette méthode réunit en quelque sorte l'analyse et la synthèse physiologiques, elle nous permet d'établir par preuve et par contre-épreuve les relations qui relient la fonction à son organe dans les manifestations cérébrales.

Lor'squ'on enlève le cerveau chezles animaux inférieurs, la fonrtion de l'organe est nécessairement supprimée; mais la persistance de la vie chez ces êtres permet au cerveau de se reformer, et, à mesure que l'organe se régénère, on voit ses fonctions reparaître.

1. Voy. Claude Bernard, Leçons sur les effets des substances toxiques. Paris, 1857. 
Cette même expérience peut également réussir chez des animaux supérieurs tels que les oiseaux, chez lesquels l'intelligence est beaucoup plus développée.

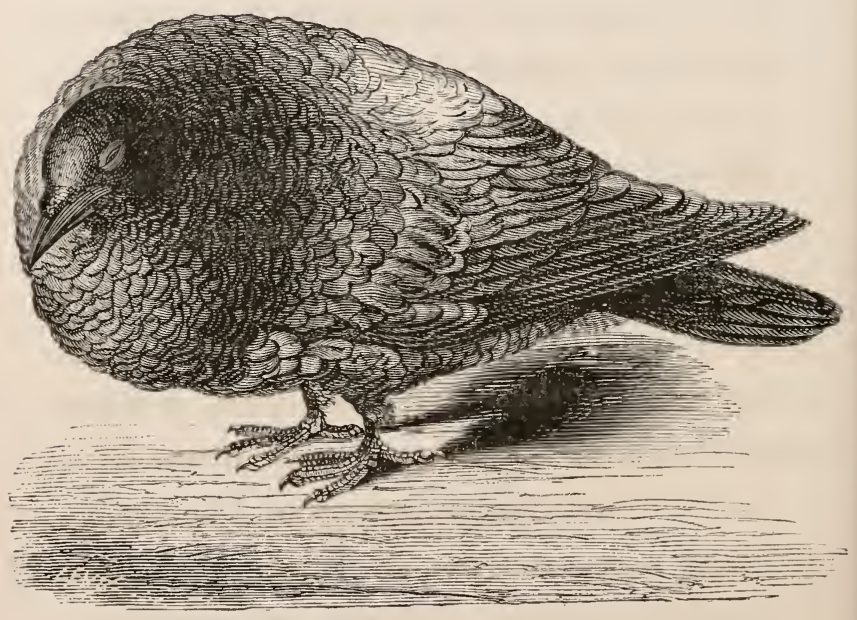

Fig. 24. Pigeon après l'ablation des lobes cérébraux ?

Les lobes cérébraux ayant été enlevés chez un pigeon par exemple (fig. 24), l'animal perd immédiatement l'usage de ses sens et la faculté

1. D après Dalton. 
de chercher sa nourriture. Toutefois, si l'on ingurgite la nourriture à l'animal, il peut survivre, parce que les fonctions nutritives sont restées intactes tant que leurs centres nerveux spéciaux ont été respectés. Peu à peu, le cerveau se régénère avec ses éléments anatomiques spéciaux, et, à mesure que cette régénération s'opère, on voit les usages des sens, les instincts et l'intelligence de l'animal revenir. Ici, je me plais à le répéter, l'expérience a été complète; il y a eu en quelque sorte analyse et synthèse de la fonction vitale, puisque la destruction successive des diverses parties du cerveau a supprimé successivement ses diverses manifestations fonctionnelles, et que la reproduction successive de ces mêmes parties a fait reparaître ces mêmes manifestations.

Il est inutile d'ajouter que la même chose arrive pour toutes les autres parties du corps susceptibles de rédintégration.

Les maladies, qui ne sont au fond que des perturbations vitales apportées par la nature au lieu d'être provoquées par la main du physiologiste, affectent le cerveau suivant les lois ordi- 
naires de la pathologie, c'est-à-dire en donnant naissance à des troubles fonctionnels qui sont toujours en rapport avec la nature et le siége de la lésion. En un mot, le cerveau a son anatomie pathologique au même titre que tous les organes de l'économie, et la pathologie cérébrale a sa symptomatologie spéciale comme celle des autres organes.

Dans l'aliénation mentale, nous voyons les troubles les plus extraordinaires de la raison, dont l'étude est une mine féconde où peuvent puiser le physiologiste et le philosophe; mais les diverses formes de la folie ou du délire ne sont que des dérangements de la fonction normale du cerveau, et ces altérations de fonctions sont, dans l'organe cérébral comme dans les autres, liées à des altérations anatomiques constantes. Si, dans beaucoup de circonstances, elles ne sont point encore connues, il faut en accuser l'imperfection seule de nos moyens d'investigation.

D'ailleurs ne voyons-nous pas certains poisons tels que l'opium, le curare, paralyser les nerfis et le cerveau sans qu'on puisse découvrir 
dans la substance nerveuse aucune altération visible? Cependant nous sommes certains que ces altérations existent, car admettre le contraire serait admettre un effet sans cause. Quand le poison a cessé d'agir, nous voyons les troubles intellectuels disparaître etl'état normal revenir. Il en est de même quand les lésions pathologiques guérissent, les troubles de l'intelligence cessent et la raison revient.

La pathologie nous fournit done encore ici une sorte d'analyse et de synthèse fonctionnelle, comme cela se voit dans les expériences de rédintégration. La maladie en effet supprime plus ou moins complétement la fonction en altérant plus ou moins complétement la texture de l'organe, et la guérison restitue la fonction en rétablissant l'état organique normal.

Si les manifestations fonctionnelles du cerveau ont été les premières qui ont attiré l'attention des philosophes, elles seront certainement les dernières qu'expliquera le physiologiste. Nous pensons que les progrès de la science moderne permettent aujourd'hui d'a- 
border la physiologie du cerveau; mais avant d'entrer dans l'étude des fonctions cérébrales, il faut bien s'entendre sur le point de départ. Ici nous avons voulu seulement poser un terme du problème, et montrer qu'il faut renoncer à l'opinion que le cerveau forme une exception dans l'organisme, qu'il est le substratum de l'intelligence et non son organe. Cette idée est non-seulement une conception surannée, mais c'est une conception antiscientifique, nuisible aux progrès de la physiologie et de la psychologie. Comment comprendre en effet qu'un appareil quelconque du domaine de la nature brute ou vivante puisse être ìe siége d'un phénomène sans en être l'instrument? On est évidemment influencé par des idées préconcues dans la question des fonctions du cerveau, et on en combat la solution par des arguments de tendance. Les uns ne veulent pas admettre que le cerveau soit l'organe de l'intelligence, parce qu'ils craignent d'être engagés par cette concession dans des doctrines matérialistes, les autres au contraire se hâtent de placer arbitrairement l'intelligence dans une cellule nerveuse 
DES FONGTIONS DU CERVEAU.

ronde ou fusiforme pour qu'on ne les taxe pas de spiritualisme.

Quant à nous, nous ne nous préoccuperons pas de ces craintes. La physiologie nous montre que, sauf la différence et la complexité plus grande des phénomènes, le cerveau est l'organe de l'intelligence au même titre que le cœur est l'organe de la circulation, que le larynx est l'organe de la voix. Nous découvrons partout une liaison nécessaire entre les organes et leurs fonctions ; c'est là un principe général auquel aucun organe du corps ne saurait se soustraire.

La physiologie doit donc, à l'exemple des sciences plus avancées, se dégager des entraves philosophiques qui gêneraient sa marche; sa mission est de rechercher la vérité avec calme et confiance, son but de l'établir d'une manière impérissable sans avoir jamais à redouter la forme sous laquelle elle peut lui apparaître.

15 mars 1872. 


\title{
DISCOURS DE RÉCEPTION
}

\author{
A L'AGADÉMIE FRANĢAISE:
}

Messieurs,

En m'appelant à l'honneur de siéger parmi vous, votre indulgence m'inspire un sentiment de reconnaissance d'autant plus vif, que la pensée même de mon insuffisance littéraire ne saurait venir le troubler. C'est l'homme de science que vous avez élu, et vos suffrages bien.

1. I. Claude Bernard, ayant été élu par l'Académie française à la place vacante par la mort de M. Flourens, y est venu prendre séance le 27 mai 1869, et a prononcé ce discours. 
veillants ont voulu honorer en moi l'Académie à laquelle j'appartiens, et perpétuer cette union des sciences et des lettres que vous n'avez cessé de consacrer par une tradition constante.

On a raison de dire que les lettres sont les sœurs aînées des sciences. C'est la loi de l'évolution intellectuelle des peuples qui ont toujours produit leurs poëtes et leurs philosophes avant de former leurs savants. Dans ce développement progressif de l'humanilé, la poésie, la philosophie et les sciences expriment les trois phases de notre intelligence, passant successivement par le sentiment, la raison et l'expérience; mais, pour que notre connaissance soit complète; il faut encore qu'une élaboration s'accomplisse en sens inverse et que l'expérience, en remontant des faits à leur cause, vienne, à son tuur, éclairer notre esprit, épurer notre sentiment et fortifier notre raison. Tout cela prouve que les lettres, la philosophie et les sciences doivent s'unir et se confondre dans la recherche des mêmes vérités; car, si, dans le langage des écoles, on sépare, sous le nom de sciences de l'esprit, les lettres et la philosophie 
des sciences proprement dites, qu'on appelle les sciences de la nature, ce serait une grave erreur de croire qu'il existe, pour cela, deux ordres de vérités distinctes ou contradictoires, les unes philosophiques ou métaphysiques, les autres scientifiques ou naturelles. Non, il ne peut y avoir au monde qu'une seule et même vérité, et cette vérité entière et absolue que l'homme poursuit avec tant d'ardeur ne sera que le résultat d'une pénétration réciproque et d'un accord définitif de toutes les sciences, soit qu'elles aient leur point de départ en nous, dans l'étude des problèmes de l'esprit humain, soit qu'elles aient pour objet l'interprétation des phénomènes de la nature, qui nous entourent.

Les sciences de l'esprit ont dù se manifester d'abord, et ont été ainsi appelées les premières à régner sur le monde; mais, aujourd'hui, dans leur gigantesque essor, les sciences de la nature remontent jusqu'à elles et veulent les pénétrer en les éclairant par l'expérience.

La physiologie, qui explique les phénomènes de la vie, constitue une science en quelque sorte intermédiaire qui prend ses racines dans 
les sciences physiques de la nature, et élève ses rameaux jusque dans les sciences philosophiques de l'esprit. Elle paraît done naturellement destinée à former le trait d'union entre les deux ordres de sciences, ayant son point d'appui solide dans les premières, et donnant aux dernières le support qui leur est indispensable. Voilà pourquoi les progrès rapides et brillants de la physiologie contemporaine excitent un intérêt général, et appellent de plus en plus l'attention sérieuse des philosophes et de tous ceux qui, comme vous, messieurs, se tiennent dans les hautes régions de la pensée et de l'esprit. C'est à cette circonstance heureuse que je suis redevable, sans aucun doute, d'avoir été distingué par vous au milieu de mes savants confrères. Vous avez perdu un physiologiste éminent, un académicien célèbre, et vous arez pensé qu'en admettant parmi vous un homme qui s'est voué à la culture de la même science, vous rendriez un hommage plus éclatant à la mémoire de celui que vous regrettez. Mais, si je m'explique ainsi l'honneur insigne que vo:s m'avez fait, je crains, d'un autre côté, de ne 
pas répondre à ce que vous attendez de moi ; car je sens, peut-être plus qu'un autre, les difficultés de juger et de louer convenablement, devant vous, mon illustre prédécesseur.

M. Flourens (Marie-Jean-Pierre) naquit à Maureilhan, arrondissementde Béziers(Hérault), le 13 avril 1794.

Heureusement doué par l'intelligence et portant au cœur l'aiguillon de la gloire et de la renommée, la nature le fit naître sous un ciel prédestiné, car l'arrondissement de Béziers a eu Ja fortune extraordinaire de compter successivement cinq de ses enfants parmi vous; et comme si une main invisible eût encore voulu tracer de plus près au jeune Flourens le sillon de sa vie, elle plaça son berceau sous le même toit oì était né Dortous de Mairan dont il devait, à un siècle de distance, occuper les deux fituteuils académiques, d'abord à l'Académie des sciences, comme secrétaire perpétuel, puis à l'Académie francaise.

Dès son enfance, M. Flourens s'était fait remarquer par l'énergie de sa volonté ainsi que par les qualités natives, de son esprit: une cu- 


\section{A L'ACADÉNIE FRANÇAISE.}

riosité intellectuelle insatiable, le désir et la recherche de ce qui était beau et distingué, une admiralion enthousiaste pour les hommes supérieurs; tels étaient les traits principaux de ce caractère d'une maturité précoce.

Arrivé à Paris en 1814, une letíre du célèbre botaniste Auguste-Pyr. de Candolle, son ancien professeur à l'école de médecine de Montpellier, l'introduisit auprès de Georges Cuvier et le placa immédiatement au foyer scientifique du temps. Dans ce nouveau milieu, son travail ardent, sa bonne tenue et la convenance parfaite de ses manières attirèrent l'attention sur lui et lui concilièrent de hautes protections. Il fuyait les tumultes du monde frivole qui éloigne de la science; mais il recherchait partout la société des hommes célèbres, et, dans quelques salons où se réunissaient des femmes éminentes ainsi que de grands savants, il sut trouver une atmosphère qui convenait à son esprit à la fois sérieux et délicat.

En moins de dix ans, M. Flourens fut membre de l'Académie des sciences, professeur au Muséum d'histoire naturelle, un des auteurs du 
Journal des savants et” secrétaire perpétuel à l'Académie des sciences. En 1840, sa réputation parvenue à son apogée recevait sa consécration la plus glorieuse; il fut élu membre de l'Académie francaise. Dès lors son horizon physiologique agrandi rayonna plus particulièrement vers le monde littéraire et vers la philosophie.

M. Flourens a été un auteur fécond, ses publications sont considérables et embrassent une période de près d’un demi-siècle. Nous ne di rons pas toutes ses recherches physiologiques; elles furent nombreuses, et dans ce genre de travaux il se montra physiologiste habile, unissant toujours les ressources d'un esprit ingénieux aux vues larges du généralisateur ${ }^{1}$. Mais,

1. Flourens, Recherches sur le développement des os et des denls. Paris, 1841, in-4, avec 12 pl.-Recherches expérimentales sur les fonctions et les propriétés du système nerveux dans les animanx vertébrés. 2* édit. Paris, 1842, in-8. - Anatomie générale de la peau et des membranes muqueuses. Paris, 1843, in-4, avec 6 pl. - Mémoires d'anatomie et de physiologie comparées. Paris, 1844, grand in 4 , arec 8 pl. - Théorie expérimentale de la formation des os. Paris, 1847, in-8, avec 7 pl. - Cours de phiysiologie comparée. De l'ontologie, ou Etude des êtres. Paris, 1856. 
à dater de 1841, il s'élève au-dessus de cette sphère purement physiologique, et entreprend la publication d'une suite de traités qu'il appelle ses ouvrages philosophiques, scientifiques et littéraires.

L'appréciation que M. Flourens a donnée des travaux et des idées d'illustres savants a beaucoup contribué à la popularité qu'il a su conquérir. En traitant des ouvrages de Fontenelle, pour lequel il avait une prédilection marquée, il le considère successivement comme philosophe et comme historien de l'Académie des sciences, et expose à ce propos d'une manière claire et rapide les principes de la philosophie expérimentale. Dans ses écrits sur l'Histoire des travaux de Georges Cuvier, sur l'Histoire des Iravaux et des idées de Buffon, M. Flourens se fait le vulgarisateur heureux des idées et des travaux de ces deux grands génies qui, comme il le dit, se complètent et se comprennent l'un par l'autre. Dans ses Éluges académiques, l'illustre secrétaire perpétuel se montre toujours soucieux de la dignité et des intérêts de l'Académie, voulant, selon son expression, écrire l'his- 
toire des sciences en écrivant celle des académiciens.

Nous ne chercherons pas à faire connaître M. Flourens par l'analyse de ses ouvrages nombreux et variés; nous nous attacherons de préférence à ses expériences originales sur le système nerveux; elles sont le trait le plus saillant de ses investigations physiologiques et forment en même temps la base de toutes ses études philosophiques.

En 1822, Magendie avait établi, à l'aide d'expériences décisives, la distinction fondamentale des nerfs moteurs et sensitifs de la moelle épinière ; c'est à peu près vers la même époque que M. Flourens présenta à l'Académie des sciences ses recherches expérimentales sur le cerveau; elles firent sensation dans le monde savant et valurent à leur jeune auteur un mémorable rapport de l'illustre Cuvier. Gall' avait eu le mérite de ramener les qualités mo-

1. Gall, Sur les fonctions du cerveau. Paris, 1825. Gall et Spurzheim, Anatomie et physiologie du système nerveux en général et du cerveau en particulien Paris 1810-1819, 4 vol., avec $100 \mathrm{pl}$. 
rales au même siége, au même organe que les facultés intellectuelles; il avait ramené la folie au même siége que la raison dont elle n'est que le trouble. Mais, à côté de ce trait de génie, comme l'appelle M. Flourens, se rencontraient des erreurs graves. Se fondant uniquement sur l'anatomie comparée, Gall pensa que les facultés intellectuelles étaient réparties dans toute la masse cérébrale, et sur cette erreur fut fondé le système des localisations phrénologiques. II. Flourens établit que l'intelligence est au contraire concentrée dans les parties les plus élevées de l'encéphale, et par ses expériences il prouva que l'ablation des hémisphères cérébraux suffit pour faire disparaître toutes les manifestations spontanées de l'instinct et de l'intelligence.

Partant de ces données expérimentales, M. Flourens aborde ensuite ses études de psychologie comparée sur l'instinct et l'intelligence des animaux; il veut, avec raison, que la psychologie embrasse l'ensemble des phénomènes intellectuels dans toute la série animale et non l'intelligence de l'homme exclusivement. 
414 DISCOURS DE RÉCEPTION

Quel admirable spectacle que celte manifestation de l'intelligence depuis l'apparition de ses premiers vestiges jusqu'à son complet épanouissement, manifestation graduée dans laquelle le physiologiste voit les diverses formes des fonctions nerveuses et cérébrales s'analyser en quelque sorte d'elles-mêmes et se répartir chez les différents animaux suivant le degré de leur organisation! D'abord, au plus bas degré, les manifestations instinctives, obscures et inconscientes; bientôt lintelligence consciente apparaissant chez les animaux d'un ordre plus élevé ; et enfin chez l'homme l'intelligence éclairée par la raison, donnant naissance à l'acte rationnellement libre, acte le plus mystérieux de l'économie animale et peut-être de la nature entière.

Dans tous les temps, les manifestations de l'intelligence ont été regardées comme des phénomènes impénétrables; mais, à mesure que la physiologie avance, elle porte ses vues de plus en plus loin. Aujourd'hui, après avoir localisé, elle veut expliquer. Elle ne se borne plus à déterminer dans les organes le siége précis des fonc- 
A L'ACADÉMIE FRANÇAISE.

tions; elle descend dans les éléments mêmes de la matière vivante, en analyse les propriétés et en déduit l'explication des phénomènes de la vie, en y découvrant les conditions de leur manifestation.

Je ne puis avoir la pensée d'entrer ici dans les arides détails de l'anatomie et de la physiologie du cerveau, cependant je vous demande la permission d'exposer rapidement quelquesuns des faits et quelques-unes des idées qui servent de jalons et de fils conducteurs à la physiologie moderne, dans les méandres encore si obscurs des phénomènes de l'intelligence.

La physiologie établit d'abord clairement que la conscience a son siége exclusivement dans les lobes cérébraux; mais, quant à l'intelligence elle-même, si on la considère d'une manière générale et comme une force qui harmonise les différents actes de la vie, les règle et les approprie à leur but, les expériences physiologiques nous démontrent que cette force n'est point concentrée dans le seul organe cérébral supérieur, et qu'elle réside au contraire, à des degrés divers, dans une foule de centres 
nerreux inconscients, échelonnés dans tout l'axe cérébro-spinal, et pouvant agir d'une facon indépendante, quoique coordonnés et subordonnés hiérarchiquement les uns aux autres.

En effet, la soustraction des lobes cérébraux chez un animal supérieur fait disparaître la conscience en laissant subsister toutes les fonctions du corps dont on a respecté les centres nerveux coordinateurs. Les fonctions de la circulation, de la respiration, continuent à s'exécuter régulièrement, sans interruption, mais elles cessent dès qu'on enlève le centre propre qui régit chacune d'elles. S'agit-il, par exemple, d'arrêter la respiration, on agira sur le centre respiratoire qui est placé dans la moelle allongée. II. Flourens a circonscrit ce centre avec une scrupuleuse précision et lui a donné le nom de noud vital, parce que sa destruction est suivie de la cessation immédiate des manifestations de la vie dans les organismes élevés. Li digestion, seulement suspendue, n'est point anéantie. L'animal, privé de la conscience et de la perception, n'a plus l'usage de ses sens et 
a perdu conséquemment la faculté de chercher sa nourriture; mais, si l'on y supplée en poussant la matière alimentaire jusqu'au fond du gosier, la digestion s'effectue parce que l'action des centres nerveux digestifs est restée intacte.

In animal dépourvu de ses lobes cérébraux n'a plus la faculté de se mouvoir spontanément et volontairement; mais si l'on substitue à l'influence de sa volonté une autre excitation, on s'assure que les centres nerveux coordinateurs des mouvements de ses membres ont conservé leur intégrité. De cette manière s'explique ce fait, étrange et bien connu, d'une grenouille décapitée qui écarte avec sa patte la pince qui la fait souffrir. On ne saurait admettre que ce mouvement si bien approprié à son but soit un acte volontaire du cerveau; il est évidemment sous la dépendance d'un centre qui, siégeant dans la moelle épinière, peut entrer en fonction, tantôt sous l'influence centrale du sens intime et de la volonté, tantôt sous l'influence d'une sensation extérieure ou périphérique.

Chaque fonction du corps possède ainsi son 
centre nerveux spécial, véritable cerveau inférieur dont la complexité correspond à celle de la fonction elle-même. Ce sont là les centres organiques ou fonctionnels qui ne sont point encore tous connus, et dont la physiologie expérimentale accroît chaque jour le nombre. Chez les animaux inférieurs, ces centres inconscients constituent seuls le système nerreux; dans les organismes élevés, ils se forment avant les centres supérieurs et président à des fonctions organiques importantes dont la nature, par prudence, suivant l'expression d'un philosophe allemand, n’a pas voulu confier le soin à la volonté.

Au-dessus des centres nerveux fonctionnels inconscients viennent se placer les centres instinclifs proprement dits. Ils sont le siége de facultés également innées dont la manifestation, quoique consciente, est involontaire, irrésistible et tout à fait indépendante de l'expérience acquise. Gall a beaucoup insisté sur les faits de ce genre, et nous pouvons en avoir tous les jours des exemples sous les yeux. Le canard qui a été couvé par une poule, et qui se jette à 
l'eau, en sortant de sa coquille, nage sans avoir rien appris ni de sa mère ni de l'expérience. La vue seule de l'eau a suffi pour réveiller son instinct. On sait encore l'histoire, rapportée par M. Flourens d'après Fr. Cuvier, d'un jeune castor, isolé au moment de sa naissance et qui, après un certain temps, commenca à construire industrieusement sa demeure.

Il y a donc des intelligences innées; on les désigne sous le nom d'insıincls. Ces facultés inférieures des centres fonctionnels et des centres instinctifs sont invariables et incapables de perfectionnement; elles sontimprimées d'avance dans une organisation achevée et immuable et sont apportées toutes faites en naissant, soit comme conditions immédiates de viabilité, soit comme moyens d'adaptation à certains modes d'existence nécessaires pour assurer le maintien et la fixité des espèces.

Mais il en est tout autrement des facultés intellectuelles supérieures; les lobes cérébraux, qui sont le siége de la conscience, ne terminent leur développement et ne commencent à manifester leurs fonctions qu'après la nais- 
sance. Il devait en être ainsi ; car, si l'organisation cérébrale eût été achevée chez le nouveau-né, l'intelligence supérieure eût été close comme les instincts, tandis qu'elle reste ouverte au contraire à tous les perfectionnements et à toutes les notions nouvelles qui s'acquièrent par l'expérience de la vie. Aussi allons-nous voir, à mesure que les fonctions des sens et du cerveau s'établissent, apparaître, dans ce dernier, des centres nerveux fonctionnels et intellectuels de nouvelle formation réellement acquis par le fait de l'éducation.

Nous désignerons sous le nom de centres les masses nerveuses qui servent d'intermédiaire aux points d'arrivée des nerfs de la sensation et aux points de départ des nerfs du mouvement. C'est dans cette substance de soudure, qui s'organise le plus tardivement, que l'exercice de la fonction vient frayer et creúser en quelque sorte les voies de communication des nerfs qui doivent se correspondre physiologiquement.

Le centre nerveux de la parole est le premicr que nous voyons se tracer chez l'enfant. Le 
sens de l'ouïe est son point de départ néces. saire; si l'organe auditif manque, le centre du langage ne se forme pas, l'enfant né sourd reste muet. Dans l'éducation des organes de la parole, il s'établit donc entre la sensation auditive et le mouvement vocal un véritable circuit nerveux qui relie les deux phénomènes dans un but fonctionnel commun. D'abord la langue balbutie; c'est par l'habitude seulement, et à l'aide . d'un exercice assez longtemps répété, que les mouvements deviennent assurés et que cette communication centrale des nerfs est rendıe facile et complète. Toutefois ce n'est qu'avec l'àge que la fonction peut s'imprimer définitirement dans l'organisation : un jeune enfant qui cesse d'entendré perd peu à peu la faculté de parler qu'il avait acquise et rederient muet, tandis que chez l'homme adulte, placé dans les mêmes conditions, il n'en est plus ainsi, parce que chez lui le centre de la parole esı fixé et le développement du cerveau achevé. A cemoment, les fonctions de ce centre acquis sont devenues vlaiment involontaires, comme si elles étaient innées; et c'est une chose remarquable que les CLAUDE bERNARD. 
actes intellectuels que nous manifestons n'atteignent réellement toute la perfection dont ils sont susceptibles que lorsque l'habitude les a imprimés dans notre organisation et les a rendus en quelque sorte indépendants de l'intelligence consciente qui les a formés et de l'attention qui les a dirigés. Chez l'orateur habile la parole est comme instinctive, et on voit, chez le musicien exercé, les doigts exécuter d'euxmêmes les morceaux les plus difficiles, sans que l'intelligence, souvent distraite par d'autres pensées, y prenne alıcune part.

Parmi tous los centres nerveux acquis, celui de la parole est sans contredit le plus important : en nous permettant de communiquer directement avec les autres hommes, il ouvre à notre esprit les plus vastes horizons. Un médecin célèbre de l'institution des sourds-muets, Itard ${ }^{1}$ nous a dépeint l'état intellectuel et moral des hommes qu'un mutisme congénital laisserait réduits à leur propre expérience. Non-

1. Itard, Traité des maladies de l'oreille et de l'audition. 2e édit. Paris, $18^{\prime}+2$. 
seulement ils subissent une véritable rétrogradation intellectuelle et morale qui les reporte en quelque sorte aux premiers temps des sociétés; mais leur esprit, fermé en partie aux notions qui nous parviennent par les sens, ne saurait se développer. Leur âme, inaccessible aux idées qui excitent l'imagination et élèvent les pensées, reste souvent muette et silencieuse parce qu'elle ne comprend pas les délicatesses du sentiment dont la parole elle-même ne parvient pas toujours à rendre toutes les nuances. Le silence est éloquent, a-t-on dit, oui, pour ceux qui savent parler et pour ceux qui, étant initiés à toutes les émotions du cœur, sentent qu'il se passe alors quelque chose en nous que les mots ne peuvent plus exprimer!

Mais ce ne sont pas seulement les mouvements de nos organes extérieurs qui deviennent automatiques; la formation de nos idées est soumise à la même loi, et, lorsqu'une idée a traversé le cerveau durant un certain temps, elle s'y grave, s'y creuse un centre et devient comme une idée innée.

Ici la physiologie vient donc justifier le sen- 
timent du poëte latin en démontrant que, pendant le jeune âge, le cerveau en voie de développement est, semblable à la cire molle, apte à recevoir toutes les empreintes qu'on lui communique, comme la jeune pousse de l'arbre prend également toutes les directions qu'on lui imprime. Plus tard, alor's que l'organisation est plus avancée, les idées et les habitudes sont, ainsi qu'on le dit, enracinées, et nous ne sommes plus maîtres ni de faire disparaître immédiatement les empreintes anciennes, ni d'en former des nouvelles.

L'organisation nerveuse de l'homme se ramène en définitive à quatre ordres de centres : les centres fonctionnels, les premiers formés, tous inconscients et dépourvus de spontanéıté; les centres instinctifs, conscients et doués de manifestations irrésistibles et fatales; les centres intellectuels, acquis d'une manière volontaire et libre, mais devenant par l'habitude plus ou inoins automatiques et involontaires. Enfin, au sommet de toutes ces manifestations, se trouve l'organe cérébral supérieur du sens intime auquel tout vient aboutir. C'est dans ce 
centre de l'unité intellectuelle qu'apparaît la conscience, qui, s'éclairant sans cesse aux lumières de l'expérience de la vie, tend à affaiblir, par le développement progressif de la raison et de la volonté, les manifestations aveugles et irrésistibles de l'instinct.

N'oublions pas que c'est aux expériences de M. Flourens que nous devons nos principales connaissances sur le siége de la conscience, et rappelons encore que l'ablation des lobes cérébraux éteint aussitôt ceflambeau de l'intelligence et de la spontanéité; la vie séparée de la conscience peut continuer sans doute, mais alors les centres nerveux inférieurs, plongés dans l'obscurité, ne sont plus capables que d'actes involontaires et purement automatiques.

Maintenant, quelle idée le physiologiste se fera-t-il sur la nature de la conscience?

II est porté d'abord à la regarder comme l'expression suprême et finale d'un certain ensemble de phénomènes nerveux et intellectuels, car l'intelligence consciente supérieure apparaît toujours la dernière, soit dans le développement de la série animale, soit dans le développe- 
ment de l'homme. Mais, dans cette évolution, comment concevoir la formation du sens intime et le passage, si gradué qu'il soit, de l'intelligence inconsciente à l'intelligence consciente? Est-ce un développement organique naturel et une intensité croissante des fonctions cérébrales qui fait jaillir l'étincelle de la conscience, restée à l'état latent, jusqu'ì ce qu'une organisation assez perfectionnée puisse permettre sa manifestation, et est-ce pour cette raison que nous royons la conscience se montrer d'autant plus lumineuse, plus active et plus libre quelle appartient à un organisme plus élevé, plus complexe, c'est-à-dire qu'elle coexiste avec des appareils intellectuels inconscients plus nombreux et plus variés? En admettant que la science vienne confirmer ces opinions, nous n'en comprendrions pas mieux pour cela, au point de vue physiologique, l'essence de la conscience que nous ne pouvons comprendre, au point de vue chimique, l'essence du feu ou de la flamme. Le physiologiste ne doit donc pas trop s'arrêter, pour le moment, à ces interprétations; il lui suffit de savoir que les phéno- 


\section{A L'AGADÉMIE FRANCAISE.}

mènes de l'intelligence et de la conscience, quelque inconnus qu'ils soient dans leur essence, quelque extraordinaires qu'ils nous apparaissent, exigent, pour se manifester, des conditions organiques ou anatomiques, des conditions physiques et chimiques qui sont accessibles à ses investigations, et c'est dans ces limites exactes qu'il circonscrit son domaine.

Partout, en effet, nous constatons une corrélation rigoureuse entre l'intensité des phénomènes physiques et chimiques et l'activité des phénomènes de la vie; c'est pourquoi il nous est possible, en agissant sur les premiers, de modifier les seconds et de les régler à notre gré. De même que les autres phénomènes vitaux, les manifestations intellectuelles sont troublées, affaiblies, éteintes ou ranimées par de simples modifications survenues dans les propriétés physiques ou chimiques du sang: il suffit de vicier ce liquide nourricier en y introduisant des anesthésiques ou certaines substances toxiques pour faire aussitôt naître le délire ou disparâ̂tre la conscience. La pensée libre, pour se manifester, exige la réunion harmonique daıs le 
cerveau de toutes ces conditions organiques, physiques et chimiques. Comment comprendre, en effet, la folie qui supprime la liberté, si on ne l'envisageait comme un trouble survenu dans ces conditions?

La tendance de la physiologie moderne est donc bien caractérisée; elle veut expliquer les phénomènes intellectuels au même titre que tous les autres phénomènes de la vie, et, si elle reconnaît avec raison qu'il y a des lacunes plus considérables dans nos connaissances, relativement aux mécanismes fonctionnels de l'intelligence, elle n'admet pas pour cela que ces mécanismes soient par leur nature ni plus ni moins inaccessibles à notre investigation que ceux de tous les autres actes vitaux.

Là, comme partout, les propriétés matérielles des tissus constituent les moyens nécessaires à l'expression des phénomènes vitaux; mais, nulle part, ces propriétés ne peuvent nous donner la raison première de l'arrangement fonctionnel des appareils. La fibre du muscle ne nous explique, par la propriété qu'elle possède de se raccourcir, que le phénomène de la con- 


\section{A L'ACADEMUE FRANCAISE.}

traction musculaire; mais cette propriété de la contractilité, qui est toujours la même, ne nous apprend pas pourquoi il existe des appareils moteurs différents, construits les uns pour produire la voix, les autres pour effectuer la respiration, etc.; et, dès lors, ne trouverait-on pas absurde de dire que les fibres musculaires de la langue et celles du larynx ont la propriété de parler ou de chanter, et celle du diaphragme la propriété de respirer? Il en est de même pour les fibres et cellules cérébrales; elles ont des pro. priétés générales d'innervation et de conductibilité, mais on ne saurait leur attribuer pour cela la propriété de sentir, de penser ou de vouloir.

Il faut donc bien se garder de confondre les propriétés de la matière avec les fonctions qu'elles accomplissent. Les propriétés de la matière n'expliquent que les phénomènes spéciaux qui en dérivent directement. Dans les œuvres de la nature et dans celles de l'homme, les, propriétés matérielles ne restent point isolées, elles sont groupées dans des organes et dans des appareils qui les coordonnent dans un but final de fonction. 
En un mot, il y a dans toutes les fonctions du corps vivant, sans exception, un côté idéal et un côté matériel. Le côté idéal de la fonction se ra'tache par sa forme à l'unité du plan de création ou de construction de l'organisme, tandis que son côté matériel répond, par son mécanisme, aux propriétés de la matière vivante. Les types des formations organiques ou fone tionnelles des êtres vivants sont développés et construits sous l'influence de forces qui leur sont spéciales; les propriétés de la matière organisée se rangent toutes, au contraire, sous l'empire des lois générales de la physique et de la chimie; elles sont soumises aux mêmes conditions d'activité que les propriétés de la matière minérale avec lesquelles elles sont en relations nécessaires et probablement équivalentes.

Les manifestations de l'intelligence ne constituent pas une exception aux autres fonctions de la vie; il n'y a aucune contradiction entre les sciences physiologiques et métaphysiques; seulement elles abordent le-même problème de l'homme intellectuel par des côtés opposés. Les 
A L'AGADÉMIE FRANCGAISE.

sciences physiologiques rattachent l'étude des facultés intellectuelles aux conditions organiques et physiques qui les expriment, tandis que les sciences métaphysiques négligent ces relations pour ne considérer les manifestations de l'âme que dans la marche progressive de l'humanité ou dans les aspirations éternelles de notre sentiment.

Nous croyons done pouvoir conclure qu'il n'y a réellement pas de ligne de séparation à établir entre la physiologie et la psychologie.

La physiologie, comme nous l'avons dit en commencant, remonte naturellement vers les sciences philosophiques, et elle sert de point d'appui immédiat à la psychologie. Elle est appelée en outre à concourir au bien-être physique de l'homme en devenant la base scientifique de l'hygiène et de la médecine; dans cette direction, la physiologie expérimentale se constitue rapidement et prend sa place parmi les sciences définies. Partout, aujourd'hui, les gouvernements aident cette jeune science de la vie dans ses moyens de développement, et elle recoit, en même temps, de toutes parts, des encourage- 
ments et des marques éclatantes d'intérêt de la part des souverains.

Les travaux de II. Flourens viensent nous montrer aussi la physiologie dans ses rapports avec la médecine. En étudiant le rôle du périoste dans la formation des os ${ }^{1}$, il a ouvert une voie que la chirurgie moderne a développée par d'importantes recherches et fécondée par d'heureuses applications. En 1861, l'Académie des sciences, voulant donner une impulsion décisive à la question de la régénération des os par le périoste, qui intéresse toute la chirurgie et plus particulièrement encore la chirurgie miljtaire, proposa sur ce sujet un grand prix de dix mille francs qui fut porté à vingt mille francs par la libéralité de l'empereur.

Il y a vingt-deux ans, la découverte de l'anesthésie par l'éther nous arriva du nouveau monde et se propagea rapidement en Europe. M. Flourens constata le premier les effets plus actifs du chloroforme, qui fut bientôt substitué à l'éther. Il a ainsi attaché son nom à cette importante dé-

1. Flourens, Théorie de la formation des os. Paris, 1847. 
couverte dont il a contribué à répandre les bienfaits.

Dans son ouvrage si populaire sur la longévite humaine, M. Flourens a cru pouroir encore s'appuyer sur la physiologie pour permettre à l'homme un siècle de vie nornıale.

Aux qualités du savant, M. Flourens joignait les qualités de l'écrivain. Par ce côté encore il a rendu service à la physiologie, il a inspiré le goût de cette science et l'a fait aimer d'un public qui, sans lui, peut-être, ne l'eût jamais connue. Il a popularisé ainsi la physiologie sans s'abaisser et l'a rendue accessible à tous par le charme du style. Sans devancer le jugement que portera tout à l'heure, sur le mérite littéraire de M. Flourens, l'une des voix les plus dignes et les plus compétentes, qu'il me soit permis de dire que l'éloquence du savant, c'est la clarté; la vérité scientifique dans sa beauté nue est toujours plus lumineuse, que parée des ornements dont notre imagination tenterait de la revêlir.

A la fois savant, écrivain, professeur et doublement académicien, M. Flourens eut une vie. 
des mieux remplies. Il devint un des physiologistes les plus renommés et les plus populaires de son temps; il dut moins encore cet éclat à son ascendant sur la jeunesse qu'à son talent d'écrivain et à la diffusion de ses travaux parmi les gens du monde. Il se consacrait entièrement à ses devoirs d'académicien et de secrétaire perpétuel de l'Académie des sciences. Il était chez lui comme dans une retraite. Absorbé par ses recherches et emporté par ses idées, il s'identifiait avec les grands hommes dont il traçait l'histoire scientifique; il habitait au Muséum d'histoire naturelle l'appartement de Buffon et s'y inspirait du souvenir de son génie.

M. Flourens parcourut une heureuse carrière, sans éprouver les luttes pénibles ni les déceptions amères qui trop souvent aigrissent et découragent l'àme. Une volonté ferme, orientée dans ses desseins par un caractère droit, un esprit élevé, secondée par une heureuse habileté et soutenue par un grand travail, le fit arriver à la renommée qu'il avait rêvée dès sa jeunesse. Il jouissait des honneurs en remplissant les devoirs de ses nombreuses fonctions; mais 
A L'AGADÉMIE FRANCAISE.

au foyer domestique il retrouvait le calme el le repos si nécessaires au savant qui travaille. Sa compagne si dérouée, si digne de le comprenlre et de l'apprécier, s'était identifiée à sa vie intellectuelle qu'elle agrandissait en lui dissimulant les soucis mêmes de l'existence. Il en était pénétré quand il répétait : " J'ai le cerveau trop occupé, il faut me faire vivre, "mais il ne goûta les douceurs de la vie intime que lorsqu'il devait bientôt les quitter. Quand la maladie l'eut forcé à une retraite complète, il disait avec quelque amertume: "Que n'ai-je plus tôt pensé à jouir de la vie de famille au lieu de la sacrifier pour d'autres qui déjà ne pensent plus à moi. „ M. Flourens fut affecté d'une paralysie qui s'empara successivement des organes de son corps; il avait parfaitement conscience de son état, et dès que le mal ne lui permit plus d'être maître de sa parole et de ses idées, il cessa de paraître dans les académies. Il suivait les progrès du mal sans que sa sérénité d'esprit en fût atteinte; il s'éteignit graduellement et mourut à Montgeron, près Paris, le 6 décembre 1867. 
M. Flourens fut un physiologiste expérimentateur; mais son nom se place aussi parmi ceux des savants qui ont abordé les généralités scientifiques.

Quelles sont les limites des sciences, de quelle nature sont les rapports qui les unissent? Ces questions restent en quelque sorte toujours présentes, et elles ont été de tous temps l'objet des méditations des esprits éminents.

On ne saurait fixer le nombre des sciences, parce qu'elles sont le résultat du morcellement successif des connaissances humaines, par notre esprit borné en une foule de problèmes séparés. Néanmoins on a distingué deux ordres de sciences : les unes partant de l'esprit pour descendre dans les phénomènes de la nature, les autres partant de l'observation de la nature pour remonter à l'esprit. Leur point de départ est différent, mais le but est le même : la recherche ét la découverte de la vérité. Ce sont les ténèbres de notre ignorance qui nous font supposer des limites entre ces deux ordres de sciences. 
A L'ACADÉMIE FRANÇAISE.

Dans l'étude des sciences, notre raison se débat entre le sentiment naturel qui nous emporte à la recherche des causes premières et l'expérience qui nous enchaîne à l'observation des causes secondes. Toutefois les luttes de ces systèmes exclusifs sont inutiles, car dans le domaine de la vérité, chaque chose doit avoir nécessairement son rôle, sa place et sa mesure.

Notre premier sentiment a pu nous faire croire qu'il était possible de construire le monde à priori, et que la connaissance des phénomènes naturels, en quelque sorte infuse en nous, s'en dégagerait par la seule force de l'esprit et du raisonnement. C'est ainsi qu'une École philosophique célèbre en Allemagne, au commencement de ce siècle, est arrivée à dire que la nature n'étant que le résultat de la pensée d'une intelligence créatrice, d'où nous émanonśs nous-mêmes, nous pouvions sans le secours de l'expérience, et pảr notre propre activité intellectuelle, retrouver les pensées du créateur. C'est là une illusion. Nous ne pourrions pas mème concevoir ainsi les inventions 
humaines, et s'il nous a été donné de connaître les lois de la nature, ce n'est qu'à la condition de les déduire par expérience de l'examen direct des phénomènes, et non des seules conceptions spéculatives de notre esprit.

- La méthode expérimentale ne se préoccupe pas de la cause première des phénomènes qui échappe à ses procédés d'investigations; c'est pourquoi elle n'admet pas qu'aucun système scientifique vienne lui imposer à ce sujet son ignorance, et elle veut que chacun reste libre dans sa manière d'ignorer et de sentir. C'est donc seulement aux causes secondes qu'elle s'adresse, parce qu' elle peut parvenir à en découvrir et à en déterminer les lois, et celles-ci n'étant que les moyens d'action ou de manifestation de la cause première, sont aussi immuables qu'elle, et constituent les lois inviolables de la nature et les bases inébranlables de la science.

Mais nos recherches n'ont point atteint les bornes de l'esprit humain; limitées par les connaissances actuelles, elles ont au-dessus d'elles l'immense région de l'inconnu qu'elles 
ne peuvent supprimer sans nuire à l'avancement même de la science.

Le connu et l'inconnu, tels sont les deux pôles scientifiques nécessaires. Le connu nous appartient et se dépose dans l'expérience des siècles. L'inconnu seul nous agite et nous tourmente et c'est lui qui excite sans cesse nos aspirations à la recherche des vérités nouvelles dont notre sentiment a l'intuition certaine, mais dont notre raison, aidée de l'expérience, veut trouver la formule scientifique.

Ce serait donc une erreur de croire que le savant qui suit les préceptes de la méthode axperimentale doive repousser toute conception à priori et imposer silence à son sentiment pour ne plus consulter que les résultats de l'expérience. Non, les lois physiologiques qui règlent les manifestations de l'intelligence humaine ne lui permettent pas de procéder autrement qu'en passant toujours et successivement par le sentiment, la raison et l'expérience; seulement, instruit par de longues déceptions et convaincu de l'inutilité des efforts de l'esprit réduit à luimême, il donne à l'expérience une influence 
prépondérante et il cherche à se prémunir contre l'impatience de connaître qui nous pousse sans cesse vers l'erreur. Il marche avec calme et sans précipitation à la recherche de la vérité; c'est la raison ou le raisonnement qui lui sert toujours de guide, mais il l'arrête, le retient et le dompte à chaque pas par l'expérience; son sentiment obéit encore, même à son insu, au besoin inné qui nous fait irrésistiblement remonter à l'origine des choses, mais ses regards restent tournés vers la nature, parce que notre ilée ne devient précise et lumineuse qu’en retournant du monde extérieur au foyer de la connaissance qui est en nous, de même que le rayon de lumière ne peut nous éclairer qu'en se réfléchissant sur les objets qui nous entourent. 


\section{TABLE DES MATIËRES}

Discours de M. Dumas aux funérailles de M. Claude

Bernard............................. 5

Clande Bernard, par Pall Bert............ 15

Du progrès dans les sciences physiologiques...... 37

Les problèmes de la physiologie gẻnérale ........ 99

Définition de la vie, les théories anciennes et la science moderne ............................. 149

La chaleur animale...................... 213

La sensibilitẻ dans le règne animal et dans le règne végétal............................ 218

Études physiologiques sur quelques poisons américains : le Gurare...................... 237

Étude sur la physiologie du Cœur............. 316

Des fonctions du cerveau.................. 367

Discours de réception à l'Académie française...... $\quad 40$ ' 



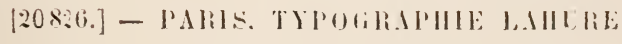

Rine de Fleurns.?

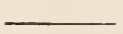





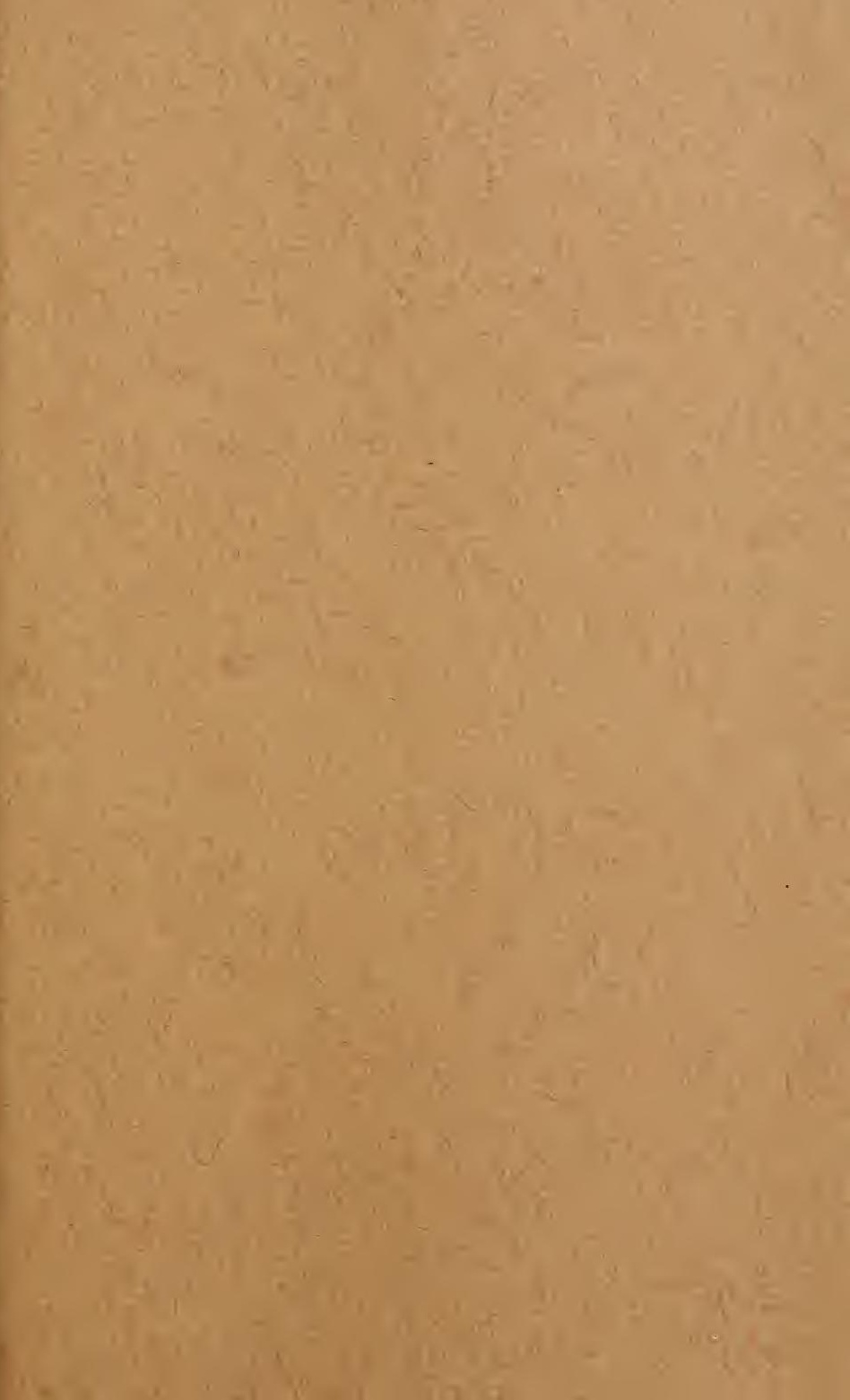





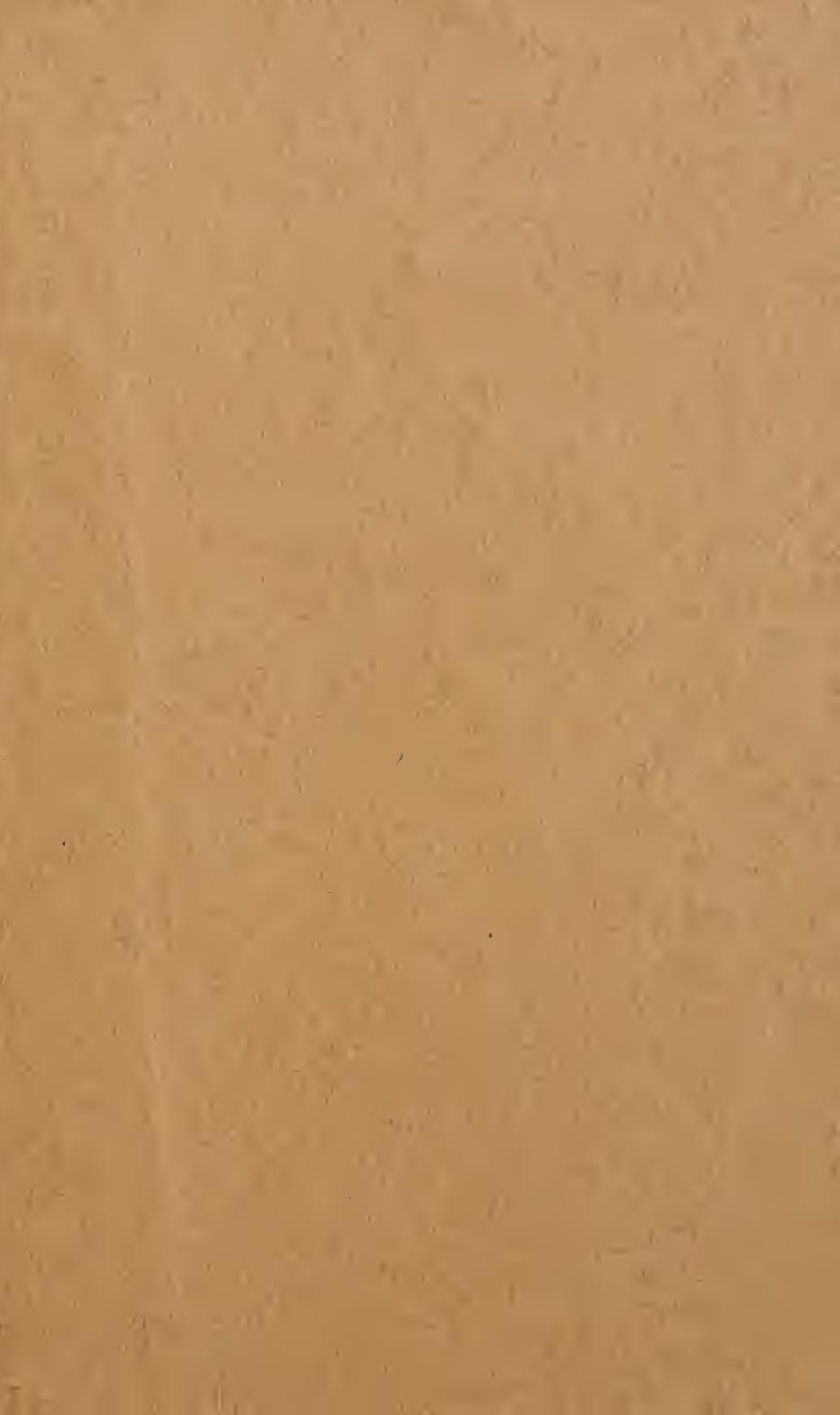


